Fellipe Garcia Marques

Modeling and Automatic Friction Compensation of Pneumatic Diaphragm Valves with Digital Positioners 
Fellipe Garcia Marques

Modeling and Automatic Friction Compensation of Pneumatic Diaphragm Valves with Digital Positioners

\section{Versão Corrigida}

(Versão original encontra-se na unidade que aloja o Programa de Pós-Graduação)

Ph.D. Thesis presented to the Escola Politécnica of the Universidade de São Paulo, to obtain the degree of Doctor of Science. 
Nome: MARQUES, Fellipe Garcia

Título: Modeling and Automatic Friction Compensation of Pneumatic Diaphragm Valves with Digital Positioners

Tese apresentada à Escola Politécnica da Universidade de São Paulo para obtenção do título de Doutor em Ciências.

Aprovado em:

Banca Examinadora

Prof. Dr.

Instituição:

Julgamento:

Prof. Dr.

Instituição:

Julgamento:

Prof. Dr.

Instituição:

Julgamento:

Prof. Dr.

Instituição:

Julgamento:

Prof. Dr.

Instituição:

Julgamento: 
Fellipe Garcia Marques

Modeling and Automatic Friction Compensation of Pneumatic Diaphragm Valves with Digital Positioners

\section{Versão Corrigida}

(Versão original encontra-se na unidade que aloja o Programa de Pós-Graduação)

Ph.D. Thesis presented to the Escola Politécnica of the Universidade de São Paulo, to obtain the degree of Doctor of Science.

Concentration Area: Systems Engineering

Advisor: Prof. Dr. Claudio Garcia

São Paulo 
Autorizo a reprodução e divulgação total ou parcial deste trabalho, por qualquer meio convencional ou eletrônico, para fins de estudo e pesquisa, desde que citada a fonte.

Este exemplar foi revisado e corrigido em relação à versão original, sob responsabilidade única do autor e com a anuência de seu orientador.

São Paulo, de de

Assinatura do autor:

Assinatura do orientador:

\section{Catalogação-na-publicação}

Marques, Fellipe

Modeling and Automatic Friction Compensation of Pneumatic Diaphragm

Valves with Digital Positioners / F. Marques -- versão corr. -- São Paulo, 2020. $163 \mathrm{p}$.

Tese (Doutorado) - Escola Politécnica da Universidade de São Paulo. Departamento de Engenharia de Telecomunicações e Controle.

1.VÁLVULAS DE CONTROLE PNEUMÁTICO 2.ATRITO 3.ESTIMAÇÃO PARAMÉTRICA 4.CONTROLE ADAPTATIVO I.Universidade de São Paulo. Escola Politécnica. Departamento de Engenharia de Telecomunicações e Controle II.t. 
To those ones who are my foundation: Mariana, Helena, Vicente, Margarete and Elza. 


\section{Acknowledgments}

This work was developed with the help of many people, many of which did not contributed directly to this thesis, but allowed me to do so. I will always be grateful for you all.

First I would like to thank my supervisor, Professor Dr. Claudio Garcia, for helping me with my technical problems and being a good listener when I needed. It was an honor working so many years with you.

The professors that helped me to improve my work in the qualification exam, Prof. Dr. Rodrigo Romano and Prof. Dr. Bruno Angélico. Thanks for presenting new paths.

The friends I have made in the laboratory, that helped me to ease such a tough journey. Thanks for the good times, laughs and technical support.

My wife, for helping me through difficult times and dedicating her time to my kids when I was not there for them.

My mother, for taking care of my grandmother.

My mother-in-law, for supporting my family.

The Brazilian Navy, for all these years of constant improvement as a professional. 
Science is the captain, and practice the soldiers.

(Leonardo da Vinci) 


\section{Abstract}

Pneumatic diaphragm valves with high friction index have the potential to decrease the control loop performance and induce limit cycles. Usually, the friction models used to model diaphragm valves are the simplest ones, mainly because there are not precise sensors available to benefit from friction models with higher complexity, as the dynamics that are modeled by more complex friction models can not be observed experimentally. In this thesis, algorithms and guidelines are designed to estimate, in open and closed loop, the valve model with simpler friction models, such as the Kano and Karnopp, as well as more complex friction models, such as the LuGre and GMS. The experimental tests, performed with two industrial valves with stem position and diaphragm pressure sensors, one with higher and the other with lower friction index, show that the GMS model has the most consistent prediction precision, with slightly better accuracy than the Karnopp model. The automatic friction compensation is developed using the Adaptive Inverse Control framework, where an offline process tunes a given controller using the previously estimated valve model. Experimental tests were performed, with several different control structures, using the Kano, Karnopp and GMS friction models to tune the controller. The results indicates that when the GMS friction model is used in the Adaptive Inverse Control framework, it is more likely to obtain optimal controller tunings, even though the Kano and Karnopp models provide pretty good tunings, as well.

Keywords: diaphragm valves, friction compensation, friction modeling, friction parameters estimation, adaptive inverse control. 


\section{Resumo}

Válvulas pneumáticas de controle com alto índice de atrito podem interferir negativamente no desempenho de malhas de controle, podendo induzir ciclos limite. Em geral, os modelos de atrito utilizados para modelar válvulas de controle são bastante simples, principalmente devido a uma indisponibilidade de sensores de alta precisão, pois não é possível observar experimentalmente os comportamentos que os modelos mais complexos são capazes de reproduzir. Neste trabalho, são desenvolvidos algoritmos e métodos para estimar, em malha aberta e fechada, o modelo da válvula utilizando tanto modelos de atrito mais simples, como o de Kano e o de Karnopp, quanto modelos de atrito mais complexos, como o de LuGre e o de GMS. Os testes experimentais, realizados utilizando duas válvulas pneumáticas industriais com sensores de posição da haste e pressão do diafragma, uma com alto e outra com baixo índice de atrito, indicam que o modelo GMS é o mais preciso, possuindo um desempenho levemente superior ao modelo de Karnopp. A compensação automática de atrito é realizada usando o método de Controle Adaptativo por Inversão, em que uma estrutura de controle é sintonizada em paralelo, utilizando o modelo estimado da válvula de controle. Inúmeros testes experimentais foram realizados, utilizando diversas estruturas de controle diferentes e empregando os modelos de atrito de Kano, Karnopp e GMS para sintonizar os controladores. Os resultados obtidos indicam que quando o modelo GMS é utilizado no método de Controle Adaptativo por Inversão, maior é a chance de se obter uma sintonia ótima do controlador, muito embora os modelos de Kano e Karnopp também sejam capazes de prover boas sintonias.

Palavras-chave: válvulas pneumáticas, compensação de atrito, modelagem de atrito, estimação de parâmetros de atrito, controle adaptativo. 


\section{List of Figures}

Figure $2.1-$ Simplified P\&ID of the FPP. . . . . . . . . . . . . . . 8

Figure 3.1 - Simplified schematic representation of a pneumatic diaphragm valve. 11

Figure $3.2-$ Friction origin. . . . . . . . . . . . . . . . . 13

Figure $3.3-$ Depiction of presliding state. . . . . . . . . . . . . . 13

Figure $3.4-$ Presliding displacement. . . . . . . . . . . . . . . . . . 14

Figure $3.5-\quad$ Decrease of $F_{s}$ with an increase in the rate of change of the external force $F . \ldots \ldots \ldots \ldots \ldots$. . . . . . . . . . . . . . . . 14

Figure 3.6 - The relationship between $F_{s}$ and the dwell time. . . . . . . . . . 14

Figure 3.7 - The friction-velocity relationship in steady state condition. . . . . . 15

Figure 3.8 - Depiction of friction lag, perceived due to different oscillation frequencies. 16

Figure 3.9 - The virtual massless elements of the GMS model. . . . . . . . . . . . 18

Figure 3.10 - A typical valve signature curve. . . . . . . . . . . . . . 22

Figure 3.11 - Excitation signal used in the simulations. . . . . . . . . . . . 25

Figure 3.12 - Comparison of continuous and discrete valve models with Kano, Karnopp, LuGre and GMS friction models. . . . . . . . . . . . . 26

Figure 3.13 - Simulation of the valve using the Kano model and comparison with the Karnopp model. . . . . . . . . . . . . . . . . . . . 26

Figure 4.1 - Simulated example for estimating $S_{0}$ a priori. . . . . . . . . . . 30

Figure 4.2 - $\quad$ I/P converter dynamics experimental data using the graphite gasket valve. . . . . . . . . . . . . . . . . . 31

Figure 4.3 - Example of excitation signal used to estimate $k$ and $F_{\text {init }}$ a priori. . . 33

Figure $4.4-\quad$ Example of excitation signal for estimating friction parameters $\left(S_{0} \approx\right.$ $25 \%$. . . . . . . . . . . . . . . . . . 35

Figure $4.5-\xi_{K}$ variation with respect to the stem velocity, for $v_{s}=5 \cdot 10^{-4} \mathrm{~m} / \mathrm{s} . \quad 37$

Figure 4.6 - Stick-slip stem position behavior simulation using valve with the Karnopp model. . . . . . . . . . . . . . . . . . . 38

Figure 4.7 - Stick-slip stem velocity behavior simulation using valve with the Karnopp model. . . . . . . . . . . . . . . . . . . 38

Figure 4.8 - Stick-slip behavior simulation, with focus in excitation signals of $5.47 \cdot 10^{-5} \mathrm{~m} / \mathrm{s}$ and $8.54 \cdot 10^{-5} \mathrm{~m} / \mathrm{s}$. . . . . . . . . . . . 39

Figure 4.9 - No stick-slip from velocities lower than $1.26 \cdot 10^{-4} \mathrm{~m} / \mathrm{s}$. . . . . . . . 39

Figure 4.10 - Slip phenomenon found at certain velocities. . . . . . . . . . . . 40

Figure 4.11 - Slip and stick-slip phenomenon found at certain velocities. . . . . . . 40

Figure 4.12 - No stick-slip from velocities higher than $1.86 \cdot 10^{-3} \mathrm{~m} / \mathrm{s}$. . . . . . . 40

Figure 4.13 - Valve with LuGre model stem position simulation, with no stick-slip behavior. . . . . . . . . . . . . . . . . . 42 
Figure 4.14 - Valve with LuGre model stem velocity simulation, with no stick-slip behavior. . . . . . . . . . . . . . . . . 42

Figure 4.15 - Valve with GMS model stem position simulation, with no stick-slip behavior. . . . . . . . . . . . . . . . . 44 44

Figure 4.16 - Valve with GMS model stem velocity simulation, with no stick-slip behavior. . . . . . . . . . . . . . . . 44

Figure 4.17 - Signals used for testing the $S_{0}$ estimation algorithm. . . . . . . . . . 51

Figure 4.18 - Signals used for testing the I/P parameter estimation algorithm. . . 52

Figure 4.19 - Signals used for testing the $k$ and $F_{\text {init }}$ estimation algorithm. . . . . . 52

Figure 4.20 - Deterioration of Kano model performance due to noise. . . . . . . . . 54

Figure 4.21 - Presliding displacement imprecision of LuGre model is negatively affected by measurement noise. . . . . . . . . . . . . . . . . . 55

Figure 4.22 - GMS with good precision in predicting presliding displacement even in the presence of noise. . . . . . . . . . . . . . . . . 55

Figure 4.23 - Valve deadband estimation in open loop. . . . . . . . . . . . . . . . 56

Figure 4.24 - Measured and estimated diaphragm pressure, using rectangular excitation. . . . . . . . . . . . . . . . 56

Figure 4.25 - Measured and estimated diaphragm pressure, using velocity excitation. 57

Figure 4.26 - Normalized diaphragm pressure and stem position for experimental open loop $k$ and $F_{\text {init }}$ estimation. . . . . . . . . . . . . . . . . . . . . 58

Figure 4.27 - Graphite gasket stem velocity during experiment for estimating $k$ and $F_{\text {init }}$ in open loop. . . . . . . . . . . . . . . . . 58

Figure 4.28 - Teflon gasket stem velocity during experiment for estimating $k$ and $F_{\text {init }}$ in open loop.

Figure 4.29 - Stribeck curve obtained for open loop $F_{\text {init }}$ estimation, used to solve Eq. (4.11).

Figure 4.30 - Filtered signals used for estimating the graphite gasket valve friction parameters, $k$ and $F_{\text {init }}$.

Figure 4.31 - Filtered signals used for estimating the teflon gasket valve friction parameters. . . . . . . . . . . . . . . . . 60

Figure 4.32 - Kano model graphite gasket valve residual using the estimation dataset. 63

Figure 4.33 - Karnopp model graphite gasket valve residual using the estimation dataset. . . . . . . . . . . . . . . . . . . . . 63

Figure 4.34 - LuGre model graphite gasket valve residual using the estimation dataset. . . . . . . . . . . . . . . . . . . . 64

Figure 4.35 - GMS model graphite gasket valve residual using the estimation dataset. 64

Figure 4.36 - Kano model graphite gasket valve residual using the validation dataset. 65

Figure 4.37 - Karnopp model graphite gasket valve residual using the validation dataset. . . . . . . . . . . . . . . . . . . . 65

Figure 4.38 - LuGre model graphite gasket valve residual using the validation dataset. 66 
Figure 4.39 - GMS model graphite gasket valve residual using the validation dataset. 66

Figure 4.40 - Kano model graphite gasket valve stem velocity prediction (450 s to $600 \mathrm{~s}$ ) using the validation dataset. . . . . . . . . . . . . . 67

Figure 4.41 - Karnopp model graphite gasket valve stem velocity prediction (450 s to $600 \mathrm{~s}$ ) using the validation dataset. . . . . . . . . . . . . 67

Figure 4.42 - LuGre model graphite gasket valve stem velocity prediction (450 s to $600 \mathrm{~s}$ ) using the validation dataset. . . . . . . . . . . . . . 67

Figure 4.43 - GMS model graphite gasket valve stem velocity prediction (450 s to $600 \mathrm{~s}$ ) using the validation dataset. . . . . . . . . . . . . . . . . . 68

Figure 4.44 - Kano and Karnopp graphite gasket valve prediction error distribution using the estimation dataset. . . . . . . . . . . . . . . . . . . . 68

Figure 4.45 - LuGre and GMS graphite gasket valve prediction error distribution using the estimation dataset.

Figure 4.46 - Kano graphite gasket valve prediction error distribution using the estimation dataset, with no scaling in $\mathrm{x}$ axis.

Figure 4.47 - Kano model teflon gasket valve residual using the estimation dataset. 71

Figure 4.48 - Karnopp model teflon gasket valve residual using the estimation dataset. 71

Figure 4.49 - LuGre model teflon gasket valve residual using the estimation dataset. 72

Figure 4.50 - GMS model teflon gasket valve residual using the estimation dataset. 72

Figure 4.51 - Kano model teflon gasket valve residual using the validation dataset. 73

Figure 4.52 - Karnopp model teflon gasket valve residual using the validation dataset. 73

Figure 4.53 - LuGre model teflon gasket valve residual using the validation dataset. 74

Figure 4.54 - GMS model teflon gasket valve residual using the validation dataset. 74

Figure 4.55 - Kano model teflon gasket valve stem velocity prediction (220s to 300 s) using the validation dataset. . . . . . . . . . . . . . .

Figure 4.56 - Karnopp model teflon gasket valve stem velocity prediction (220s to $300 \mathrm{~s}$ ) using the validation dataset. . . . . . . . . . . . . . . 75

Figure 4.57 - LuGre model teflon gasket valve stem velocity prediction (220s to 300 s) using the validation dataset. . . . . . . . . . . . . . .

Figure 4.58 - GMS model teflon gasket valve stem velocity prediction (220s to 300 s) using the validation dataset. . . . . . . . . . . . . . 76

Figure 4.59 - Kano and Karnopp teflon gasket valve prediction error distribution using the estimation dataset. . . . . . . . . . . . . . . . . . 76

Figure 4.60 - LuGre and GMS teflon gasket valve prediction error distribution using the estimation dataset. . . . . . . . . . . . . . . . . . . . . 77

Figure 5.1 - Scheme of the diaphragm valve with digital positioner. . . . . . . . 78

Figure 5.2 - Scheme of valve diagnostics by analyzing the difference between measured and simulated variables. . . . . . . . . . . . . . . 79

Figure 5.3 - Residual distribution for plant with factory performance and deviation

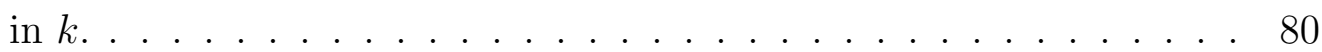


Figure 5.4 - Residual distribution for plant with deviation in $F_{\text {init }}$ and friction parameters. . . . . . . . . . . . . . . . . . . 81

Figure 5.5 - Closed loop excitation signal example. . . . . . . . . . . . . . . 84

Figure 5.6 - Signals used to estimate the I/P model parameters and $S_{0}$ in the simulated closed loop case. . . . . . . . . . . . . . . . . . . 95

Figure 5.7 - Valve signature curve with only nonzero stem velocities used to estimate $S_{0}$ in the simulated closed loop case. . . . . . . . . . 96

Figure $5.8-\quad$ Signals used to estimate $k, F_{\text {init }}$ and $\theta_{f}$ in the simulated closed loop case. . . . . . . . . . . . . . . . . . 996

Figure 5.9 - Signals used to estimate the graphite gasket valve I/P model parame-

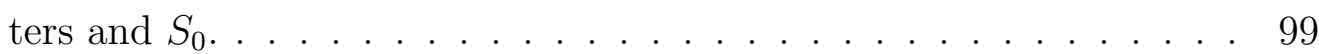

Figure 5.10 - Signals used to estimate the teflon gasket valve I/P model parameters and $S_{0} \ldots \ldots \ldots \ldots \ldots$

Figure 5.11 - Valves signature curves with only nonzero stem velocities used to estimate $S_{0} \ldots \ldots \ldots \ldots \ldots$. . . . . . . . . . . . . . . . . . . . .

Figure 5.12 - Kano model graphite gasket valve residual with model parameters estimated in closed loop using the estimation dataset. . . . . . . . . 103

Figure 5.13 - Karnopp model graphite gasket valve residual with model parameters estimated in closed loop using the estimation dataset.

Figure 5.14 - LuGre model graphite gasket valve residual with model parameters estimated in closed loop using the estimation dataset. . . . . . . . . 104

Figure 5.15 - GMS model graphite gasket valve residual with model parameters estimated in closed loop using the estimation dataset.

Figure 5.16 - Kano model graphite gasket valve residual with model parameters estimated in closed loop using the validation dataset. . . . . . . . . 105

Figure 5.17 - Karnopp model graphite gasket valve residual with model parameters estimated in closed loop using the validation dataset. . . . . . . . . . 105

Figure 5.18 - LuGre model graphite gasket valve residual with model parameters estimated in closed loop using the validation dataset. . . . . . . . . . 106

Figure 5.19 - GMS model graphite gasket valve residual with model parameters estimated in closed loop using the validation dataset. . . . . . . . . 106

Figure 5.20 - Kano model graphite gasket valve stem velocity prediction (450 s to $600 \mathrm{~s}$ ) with model parameters estimated in closed loop using the open loop validation dataset. . . . . . . . . . . . . . . . . . . 107

Figure 5.21 - Karnopp model graphite gasket valve stem velocity prediction (450 s to $600 \mathrm{~s}$ ) with model parameters estimated in closed loop using the open loop validation dataset.

Figure 5.22 - LuGre model graphite gasket valve stem velocity prediction (450 s to $600 \mathrm{~s}$ ) with model parameters estimated in closed loop using the open loop validation dataset. . . . . . . . . . . . . . . 107 
Figure 5.23 - GMS model graphite gasket valve stem velocity prediction (450 s to $600 \mathrm{~s}$ ) with model parameters estimated in closed loop using the open loop validation dataset. . . . . . . . . . . . . . . 108

Figure 5.24 - Kano model teflon gasket valve residual with model parameters estimated in closed loop using the estimation dataset. . . . . . . . . 109

Figure 5.25 - Karnopp model teflon gasket valve residual with model parameters estimated in closed loop using the estimation dataset. . . . . . . . 110

Figure 5.26 - LuGre model teflon gasket valve residual with model parameters estimated in closed loop using the estimation dataset. . . . . . . . . 110

Figure 5.27 - GMS model teflon gasket valve residual with model parameters estimated in closed loop using the estimation dataset.

Figure 5.28 - Kano model teflon gasket valve residual with model parameters estimated in closed loop using the validation dataset. . . . . . . . . . 111

Figure 5.29 - Karnopp model teflon gasket valve residual with model parameters estimated in closed loop using the validation dataset. . . . . . . . . . 112

Figure 5.30 - LuGre model teflon gasket valve residual with model parameters estimated in closed loop using the validation dataset. . . . . . . . . . 112

Figure 5.31 - GMS model teflon gasket valve residual with model parameters estimated in closed loop using the validation dataset. . . . . . . . . . 113

Figure 5.32 - Kano model teflon gasket valve stem velocity prediction (220 s to $300 \mathrm{~s}$ ) with model parameters estimated in closed loop using the open loop validation dataset. . . . . . . . . . . . . . . . . . . . . . . 113

Figure 5.33 - Karnopp model teflon gasket valve stem velocity prediction $(220 \mathrm{~s}$ to $300 \mathrm{~s}$ ) with model parameters estimated in closed loop using the open loop validation dataset. . . . . . . . . . . . . . . . . 114

Figure 5.34 - LuGre model teflon gasket valve stem velocity prediction (220 s to $300 \mathrm{~s}$ ) with model parameters estimated in closed loop using the open loop validation dataset. . . . . . . . . . . . . . . . . . . 114

Figure 5.35 - GMS model teflon gasket valve stem velocity prediction (220 s to $300 \mathrm{~s}$ ) with model parameters estimated in closed loop using the open loop validation dataset. . . . . . . . . . . . . . . . . . . . . . 114

Figure 6.1 - Adaptive Inverse Control framework example for the diaphragm valve position controller tuning. . . . . . . . . . . . . . . 117

Figure 6.2 - Example of the excitation signal used to obtain the optimal parameters of $C_{p}$. . . . . . . . . . . . . . . . . . . . . . . . . . . . . . 119

Figure 6.3 - Servo test example, using the PID controller tuned with the Kano $\mathrm{OL}$ and $\tau_{\mathrm{I} / \mathrm{P}_{r}}$ estimated model.

Figure 6.4 - Regulatory test example, using the PID controller tuned with the Karnopp OL and $\tau_{\mathrm{I} / \mathrm{P}_{s}}$ estimated model. . . . . . . . . . . . . 122 
Figure 6.5 - $\quad$ Aleatory test example, using the PID controller tuned with the GMS CL estimated model. . . . . . . . . . . . . . . . . . . . . . 122

Figure 6.6 - Comparison of the tuning performance of the AIC method in the initial PID test, grouping data by model estimation type.

Figure 6.7 - Comparison of iae $_{Q}$, among the estimated friction models. . . . . . . 124

Figure 6.8 - Comparison of iae $_{x}$, among the estimated friction models. . . . . . . 124

Figure 6.9 - Comparison of std (OP), among the estimated friction models. . . . . 124

Figure 6.10 - Aleatory experiment extract using PID tuned with open loop estimated Karnopp and GMS models in the AIC framework (with $\hat{\tau}_{\mathrm{I} / \mathrm{P}_{r}}$ as the I/P model time constant). . . . . . . . . . . . . . . . . . . 125

Figure 6.11 - Aleatory experiment extract using PID tuned with open loop estimated Kano and GMS models in the AIC framework (with $\hat{\tau}_{\mathrm{I} / \mathrm{P}_{r}}$ as the I/P model time constant). . . . . . . . . . . . . . . . . . . 125

Figure 6.12 - Comparison of tuning performance using DS or AIC methods, in the robustness test. . . . . . . . . . . . . . . . . . 130

Figure 6.13 - Comparison of the AIC tuning performance when using different estimated models, in the robustness test. . . . . . . . . . . . . . . 130

Figure 6.14 - Comparison, for each experiment, of the friction models tuning performance, in the robustness test.

Figure 6.15 - Comparison of the iae $_{Q}$ index, using the GMS model in the AIC framework, for each controller, in the robustness test. . . . . . . . . . 131

Figure 6.16 - Comparison of the iae $_{Q}$ index, using the Kano model in the AIC framework, for each controller, in the robustness test. . . . . . . . . . 132

Figure 6.17 - Comparison of the iae $_{Q}$ index, using the Karnopp model in the AIC framework, for each controller, in the robustness test. . . . . . . . . . 132

Figure 6.18 - Comparison of the std(OP) index, using the GMS model in the AIC framework, for each controller, in the robustness test. . . . . . . . . . 132

Figure 6.19 - Comparison of the std(OP) index, using the Kano model in the AIC framework, for each controller, in the robustness test. . . . . . . . . 133

Figure 6.20 - Comparison of the std(OP) index, using the Karnopp model in the AIC framework, for each controller, in the robustness test. . . . . . . 133

Figure 6.21 - AIC and DS tuning performance comparison of the PID and PID with CF control structures, in the robustness test.

Figure 6.22 - AIC and DS tuning performance comparison of the PID with CF and Knocker, and the PID with CF and CR, in the robustness test. . . . 134

Figure 6.23 - AIC and DS tuning performance comparison of the fuzzy PID control structure, in the robustness test.

Figure 6.24 - Extract of the aleatory experiment using the PID with CF and CR friction compensation, tuned with the Karnopp and GMS models. . . 135 
Figure 6.25 - Extract of the aleatory experiment using the PID with CF and CR friction compensation, tuned with the Kano and GMS models. . . . 135

Figure 6.26 - Comparison of tuning performance when the open and closed loop estimated valve models are used in the AIC framework. . . . . . . . 140

Figure 6.27 - Comparison of the controller tuning performance, for each open loop estimated friction model used in the AIC framework. . . . . . . . . . 140

Figure 6.28 - Comparison of the controller tuning performance, for each closed loop estimated friction model used in the AIC framework. . . . . . . . . . 141

Figure 6.29 - Comparison of the controller tuning performance, separated by experiment type, for each open loop estimated friction model used in the AIC.

Figure 6.30 - Comparison of the tuning performance when the open loop estimated GMS model is used in the AIC framework. . . . . . . . . . . . . . . 142

Figure 6.31 - Comparison of the tuning performance when the open loop estimated Kano model is used in the AIC framework. . . . . . . . . . . . . . . 143

Figure 6.32 - Comparison of the tuning performance when the open loop estimated Karnopp model is used in the AIC framework. . . . . . . . . . . . . 143

Figure 6.33 - Comparison of the tuning performance when the closed loop estimated GMS model is used in the AIC framework. . . . . . . . . . . . . . . 143

Figure 6.34 - Comparison of the tuning performance when the closed loop estimated Kano model is used in the AIC framework. . . . . . . . . . . . . . . 144

Figure 6.35 - Comparison of the tuning performance when the closed loop estimated Karnopp model is used in the AIC framework. . . . . . . . . . . . . 144

Figure 6.36 - Comparison of the controller tuning performance when different open loop estimated friction models are used in the AIC framework. . . . 145

Figure 6.37 - Comparison of the controller tuning performance when different closed loop estimated friction models are used in the AIC framework. . . . 145

Figure 6.38 - Extract of the servo experiment using the PID with CF and CR, tuned using the open loop estimated GMS and Kano models in the AIC framework. . . . . . . . . . . . . . . . . . . . . . 146

Figure 6.39 - Extract of the servo experiment using the PID with CF and CR, tuned using the open loop estimated Kano and Karnopp models in the AIC framework. . . . . . . . . . . . . . . . . . . 146

Figure 6.40 - Extract of the servo experiment using the FFPID, tuned using the open loop estimated GMS and Kano models in the AIC framework. . 146

Figure 6.41 - Extract of the servo experiment using the FFPID, tuned using the open loop estimated Kano and Karnopp models in the AIC framework.147

Figure 6.42 - Extract of the servo experiment using the ISMC, tuned using the open loop estimated GMS and Kano models in the AIC framework. . 147 
Figure 6.43 - Extract of the servo experiment using the ISMC, tuned using the open loop estimated Kano and Karnopp models in the AIC framework.147

Figure 6.44 - Comparison of the iae $_{Q}$ index, in the teflon gasket valve test. . . . . 149

Figure 6.45 - Comparison of the std(OP) index, in the teflon gasket valve test. . . 150

Figure 6.46 - Comparison of the std(OP) index, excluding the ISMC controller. . . 150

Figure 6.47 - Extract of the servo experiment using the PID and PID with CF and CR, tuned using the DS method. . . . . . . . . . . . . 150

Figure 6.48 - Extract of the servo experiment using the PID with CF and CR, tuned using the DS method and the AIC with the open loop estimated Kano model. . . . . . . . . . . . . . . . . . . . 151

Figure 6.49 - Extract of the servo experiment using the PID with CF and CR and FFPID, tuned using the the AIC with the open loop estimated Kano model. . . . . . . . . . . . . . . . . . 151

Figure 6.50 - Extract of the servo experiment using the FFPID and ISMC, tuned using the the AIC with the open loop estimated Kano model. . . . . 151 


\section{List of Tables}

Table 3.1 - Valve parameters used in the simulations. . . . . . . . . . . . . . 24

Table 3.2 - Friction parameters used in the simulations. . . . . . . . . . . . . 25

Table 3.3 - Continuous and discrete valve models comparison. . . . . . . . . . . 27

Table 4.1 - Kano parameter bounds. . . . . . . . . . . . . . . 36

Table 4.2 - Karnopp parameter bounds. . . . . . . . . . . . . . . . . 41

Table 4.3 - LuGre parameter bounds, an extension of Table 4.2. . . . . . . . . . 43

Table 4.4 - GMS parameter bounds, an extension of Table 4.2. . . . . . . . . . . 44

Table 4.5 - GA optimization LuGre parameter estimation results. . . . . . . . . . 46

Table 4.6 - GA optimization LuGre parameter estimation error. . . . . . . . . . . 47

Table 4.7 - DE optimization LuGre parameters estimation results. . . . . . . . . . 47

Table 4.8 - DE optimization LuGre parameters estimation error. . . . . . . . . . . 47

Table 4.9 - MS optimization LuGre parameter estimation results. . . . . . . . . . . 48

Table 4.10 - MS optimization LuGre parameters estimation error. . . . . . . . . . . 48

Table 4.11 - SNL optimization LuGre parameter estimation results. . . . . . . . . . 49

Table 4.12 - SNL optimization LuGre parameter estimation error. . . . . . . . . . . 49

Table 4.13 - GDES estimation results. . . . . . . . . . . . . . . . 50

Table 4.14 - GDES Kano parameter estimation error. . . . . . . . . . . . . . . 50

Table 4.15 - GDES Karnopp parameter estimation error. . . . . . . . . . . . . . . 50

Table 4.16 - GDES LuGre parameter estimation error. . . . . . . . . . . . . . 50

Table 4.17 - GDES GMS parameter estimation error. . . . . . . . . . . . . 51

Table 4.18 - Open loop valve parameters estimation error in simulated case. . . . . 53

Table 4.19 - Friction parameter estimation test with simulated data results. . . . . 53

Table 4.20 - Kano parameter estimation error, test with simulated data. . . . . . . 54

Table 4.21 - Karnopp parameter estimation error, test with simulated data. . . . . 54

Table 4.22 - LuGre parameter estimation error, test with simulated data. . . . . . . 54

Table 4.23 - GMS parameter estimation error, test with simulated data. . . . . . . 54

Table 4.24 - Open loop valve parameter estimation with experimental data. . . . . 57

Table 4.25 - Open loop graphite gasket valve model estimation performance. . . . . 61

Table 4.26 - Open loop teflon gasket valve model estimation performance. . . . . . 61

Table 4.27 - Open loop Kano estimated parameters. . . . . . . . . . . . . . . . . 61

Table 4.28 - Open loop Karnopp estimated parameters. . . . . . . . . . . . . . . . 61

Table 4.29 - Open loop LuGre estimated parameters. . . . . . . . . . . . . . . . . 61

Table 4.30 - Open loop GMS estimated parameters. . . . . . . . . . . . . . . 62

Table 4.31 - Statistical measures of the graphite gasket valve prediction. . . . . . . 70

Table 4.32 - Statistical measures of the teflon gasket valve prediction. . . . . . . . 77

Table 5.1 - Statistical measures of simulated defective valves of Figures 5.3a to 5.4b. 81 
Table 5.2 - Closed loop I/P and valve signature curve parameters estimation in the simulated case. . . . . . . . . . . . . . . . . . . . . 95

Table 5.3 - Friction parameters estimation test with simulated data results. . . . . 96

Table 5.4 - Closed loop friction parameter estimation test results with simulated data. . . . . . . . . . . . . . . . . . . . 997

Table 5.5 - Closed loop Kano parameter estimation error in a test with simulated data. . . . . . . . . . . . . . . . . . . . 997

Table 5.6 - Closed loop Karnopp parameter estimation error in a test with simulated data. . . . . . . . . . . . . . . . . . . . 997

Table 5.7 - Closed loop LuGre parameter estimation error in a test with simulated data. . . . . . . . . . . . . . . . . . . . 997

Table 5.8 - Closed loop GMS parameters estimation error, test with simulated data. 98

Table 5.9 - Closed loop I/P model and $S_{0}$ parameter estimation deviation from the open loop case. . . . . . . . . . . . . . . . . . . . . . . . . . . . . . . 999

Table 5.10 - Closed loop graphite gasket valve model estimation performance. . . . 101

Table 5.11 - Closed loop teflon gasket valve model estimation performance. . . . . . 101

Table 5.12 - Closed loop Kano parameter estimation deviation from open loop tests. 101

Table 5.13 - Closed loop Karnopp parameter estimation deviation from open loop tests. . . . . . . . . . . . . . . . . . . 101

Table 5.14 - Closed loop LuGre parameter estimation deviation from open loop tests.102 Table 5.15 - Closed loop GMS parameter estimation deviation from open loop tests. 102 Table 5.16 - Statistical measures of the graphite gasket valve prediction using parameters estimated in closed loop. . . . . . . . . . . . . . . . . . . 108

Table 5.17 - Statistical measures of the graphite gasket valve prediction using parameters estimated in closed loop. . . . . . . . . . . . . . . . . 115

Table 6.1 - PID controller parameter search space. . . . . . . . . . . . . . . . 120

Table 6.2 - PID tunings for each estimated model used in the AIC framework. . . 121

Table 6.3 - CF method parameter search space. . . . . . . . . . . . . . 126

Table 6.4 - CF method parameter search space. . . . . . . . . . . . . . . 127

Table 6.5 - Fuzzy PID controller parameter search space. . . . . . . . . . . . . . 128

Table 6.6 - PID tunings in the robustness test. . . . . . . . . . . . . . . . . 129

Table 6.7 - PID with CF tunings in the robustness test. . . . . . . . . . . . . . 129

Table 6.8 - PID with CF and Knocker friction compensation tunings in the robustness test. . . . . . . . . . . . . . . . . . . . . 129

Table 6.9 - PID with CF and CR friction compensation tunings in the robustness test. . . . . . . . . . . . . . . . . . . 129

Table 6.10 - Fuzzy PID tunings in the robustness test. . . . . . . . . . . . . . . . 129

Table 6.11 - ISMC parameter search space. . . . . . . . . . . . . . . . 137

Table 6.12 - FFPID parameter search space. . . . . . . . . . . . . . . . 138 
Table 6.13 - PID with CF and CR friction compensation tunings in the ISMC and FFPID tests. . . . . . . . . . . . . . . . . . . . 139

Table 6.14 - ISMC tunings in the ISMC and FFPID tests. . . . . . . . . . . . . 139

Table 6.15 - FFPID tunings in the ISMC and FFPID tests. . . . . . . . . . . . 139

Table 6.16 - PID with CF and CR friction compensation tunings in the teflon gasket valve test. . . . . . . . . . . . . . . . . . . . . 148

Table 6.17 - ISMC tunings in the teflon gasket valve test. . . . . . . . . . . . 148

Table 6.18 - FFPID tunings in the teflon gasket valve test. . . . . . . . . . . . . . 149 


\title{
Symbology and Abbreviations
}

\section{Abbreviations and Acronyms}

\author{
AIC Adaptive Inverse Control; \\ CF Control Freezing Algorithm; \\ CR Constant Reinforcement Compensator; \\ DC Direct Current; \\ DCS Digital Control System; \\ DE Differential Evolution Optimization; \\ DS Direct Synthesis; \\ EPUSP Escola Politécnica of the University of São Paulo; \\ FFPID Feed Forward PID with Integral Deadband; \\ FPGA Field Programmable Gate Array; \\ FPP Flow Pilot Plant; \\ GA Genetic Algorithm Optimization; \\ GDES Grid Search with Differential Evolution and Simplex Optimization; \\ GMS Generalized Maxwell-Slip; \\ I/P Current to Pressure; \\ IAE Integral Absolute Error; \\ IFAC International Federation of Automatic Control; \\ IPCL Industrial Process Control Laboratory; \\ IRAE Integral Root Absolute Error; \\ ISMC Integrative Sliding Mode Control; \\ LSE Least Squares Estimator; \\ LVDT Linear Variable Differential Transformer; \\ LuGre Leuven-Grenoble; \\ MS Monte Carlo with Simplex Optimization; \\ MSE Mean Squared Error; \\ OP Controller Output; \\ PID Proportional Integral Derivative Controller; \\ P\&ID Piping \& Instrumentation Diagram; \\ SNL Successive Nonlinear Linear Regression; \\ SNR Signal to Noise Ratio; \\ SP Set Point.
}




\section{Operators and Mathematical Notation}

$\begin{array}{ll}\dot{x} & \text { Time derivative of } x ; \\ \ddot{x} & \text { Second order time derivative of } x ; \\ \hat{x} & \text { Estimated value of } x ; \\ \operatorname{sgn}(\cdot) & \text { Signal function; } \\ \min (\cdot) & \text { Minimum function; } \\ \max (\cdot) & \text { Maximum function; } \\ \operatorname{sat}(\cdot) & \text { Saturation function; } \\ \mu(\cdot) & \text { Mean value function; } \\ \operatorname{std}(\cdot) & \text { Standard deviation function; } \\ \mu_{3}(\cdot) & \text { Third order moment function; } \\ E(\cdot) & \text { Expectation function; } \\ q & \text { Time shift operator; } \\ n & \text { Discrete time; } \\ t & \text { Continuous time; } \\ f i t & \text { Goodness of fit index; } \\ \mathbb{R}^{N} & \text { Set of real numbers of dimension } N ; \\ \mathbb{N} & \text { Set of natural numbers; }\end{array}$

$\arg \min V$ Function that returns the argument that minimizes the function $\mathrm{V}$.

\section{Valve and Friction Model Symbols}

$\begin{array}{ll}x & \text { Valve stem position; } \\ P & \text { Diaphragm air pressure; } \\ \text { OP } & \text { I/P converter input signal; } \\ T_{s} & \text { Discrete model sampling time; } \\ S_{a} & \text { Diaphragm cross sectional area; } \\ m & \text { Pneumatic diaphragm valve moving parts mass; } \\ k & \text { Valve spring constant; } \\ F_{\text {init }} & \text { Valve initial force; } \\ F_{f} & \text { Friction force; } \\ P_{\min } & \text { Minimum diaphragm pressure; } \\ P_{\max } & \text { Maximum diaphragm pressure; } \\ x_{\min } & \text { Valve stem minimum position; } \\ x_{\max } & \text { Valve stem maximum position; } \\ K_{P_{g}} & \text { I/P converter pressure gain; } \\ \tau_{\mathrm{I} / \mathrm{P}} & \text { I/P converter model time constant; } \\ a_{0} & \text { I/P converter discrete model parameter; }\end{array}$


$b_{0} \quad \mathrm{I} / \mathrm{P}$ converter discrete model parameter;

$b_{1} \quad \mathrm{I} / \mathrm{P}$ converter discrete model parameter;

$F_{s} \quad$ Static friction force;

$F_{c} \quad$ Coulomb friction force;

$F_{v} \quad$ Viscous friction parameter;

$v_{s} \quad$ Stribeck velocity;

DV Stem velocity that defines when the Karnopp model is in the presliding state;

$z \quad$ LuGre model internal state;

$\sigma_{0} \quad$ LuGre model elastic coefficient;

$\sigma_{1} \quad$ Lugre model friction damping coefficient;

$i \quad i$ th Maxwell element of the GMS model;

$\psi_{i} \quad i$ th internal state of the GMS model;

$N_{M_{e}} \quad$ Number of Maxwell elements of the GMS model;

$\kappa_{i} \quad$ GMS model elastic coefficient of the $i$ th Maxwell element;

$\nu_{i} \quad$ GMS model friction damping coefficient of the $i$ th Maxwell element;

$\alpha_{i} \quad$ GMS model contribution coefficient of the $i$ th Maxwell element;

C GMS model attraction constant;

$S \quad$ Kano model deadband parameter;

$J \quad$ Kano model slip band parameter;

D Kano model initial force parameter;

$x_{\max _{P}} \quad$ Kano model maximum stem position when the diaphragm pressure is $P_{\max }$;

$x_{\min _{P}} \quad$ Kano model minimum stem position when the diaphragm pressure is $P_{\text {min }}$;

$\theta_{\mathrm{I} / \mathrm{P}} \quad$ Vector that groups the I/P converter model parameters;

$\theta_{f} \quad$ Vector that groups the friction model parameters;

$\hat{\tau}_{\mathrm{I} / \mathrm{P}_{r}} \quad \mathrm{I} / \mathrm{P}$ model estimated time constant using ramps as excitation signal;

$\hat{\tau}_{\mathrm{I} / \mathrm{P}_{s}} \quad \mathrm{I} / \mathrm{P}$ model estimated time constant using steps as excitation signal;

$V_{n_{f}} \quad$ Stem position prediction residual function.

\section{Valve Stem Position Controller Symbols}

$\begin{array}{ll}x_{d} & \text { Desired stem position signal; } \\ g_{x} & \text { Stem position deviation gain; } \\ g_{u} & \text { Control effort gain; } \\ \theta_{p} & \text { Stem position controller parameter vector; } \\ \theta_{p}{ }^{*} & \text { Optimal tuning of the stem position controller parameter vector; } \\ J_{C}\left(\theta_{p}\right) & \text { AIC cost function for a given } \theta_{p} ; \\ C_{H} & \text { Hydraulic system controller; } \\ S P_{H} & \text { Hydraulic system controller set point; } \\ K_{H} & \text { Hydraulic system controller proportional gain; }\end{array}$


$K_{i H} \quad$ Hydraulic system controller integral gain;

$C_{p} \quad$ Valve stem position controller;

$S P \quad$ Stem position controller set point;

$K_{p} \quad$ Position controller proportional gain;

$K_{i} \quad$ Position controller integral gain;

$K_{d} \quad$ Position controller derivative gain;

$\tau_{d f} \quad$ Position controller derivative filter time constant;

$T_{s_{C_{p}}} \quad$ Position controller sampling time;

$e_{l} \quad \mathrm{CF}$ algorithm low error threshold;

$e_{h} \quad \mathrm{CF}$ algorithm high error threshold;

$t_{e_{l}} \quad \mathrm{CF}$ algorithm time with low error threshold;

$t_{e_{h}} \quad \mathrm{CF}$ algorithm time with high error threshold;

$t_{k_{p}} \quad$ Knocker compensator pulse signal period;

$d_{k_{p}} \quad$ Knocker compensator signal duty cycle;

$a_{k n} \quad$ Knocker compensator signal amplitude;

$a_{C R} \quad$ CR compensator signal amplitude;

$s d_{\hat{e}} \quad$ Fuzzy PID controller standard deviation parameter of the control error;

$s d_{\hat{\hat{e}}} \quad$ Fuzzy PID controller standard deviation parameter of the time derivative of the control error;

$K_{i 1} \quad$ Fuzzy PID controller integral gain when the control error and its time derivative are nonzero;

$K_{i 2} \quad$ Fuzzy PID controller integral gain when the control error is zero and its time derivative is nonzero;

$K_{i 3} \quad$ Fuzzy PID controller integral gain when the control error is nonzero and its time derivative is zero;

$K_{i 4} \quad$ Fuzzy PID controller integral gain when the control error and its time derivative are zero;

$\lambda_{i s m c} \quad$ ISMC sliding surface time constant;

$k_{i s m c} \quad$ ISMC gain;

$\Theta_{i s m c} \quad$ ISMC sliding surface dead zone;

$\tau_{\text {isme }} \quad$ ISMC control error filter time constant;

$e_{I_{t h}} \quad$ FFPID error threshold to activate the integral action;

$k_{f f} \quad$ FFPID feed forward gain. 


\section{Contents}

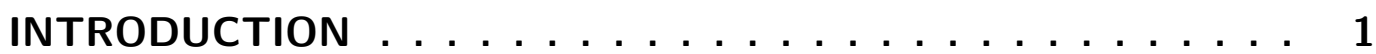

1.1

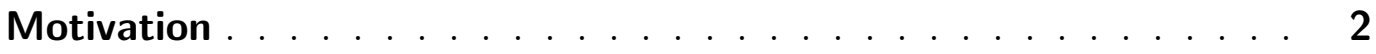

1.2

Objectives

$1.3 \quad$ Literature Review . . . . . . . . . . . . . . . 3

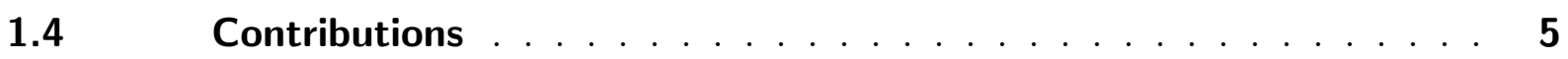

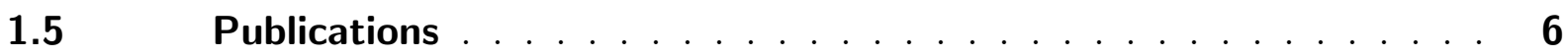

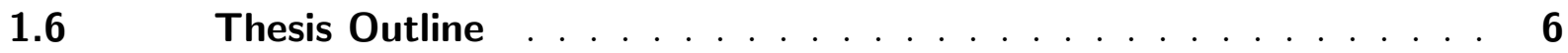

2 THE FLOW PILOT PLANT $\ldots \ldots \ldots \ldots \ldots$

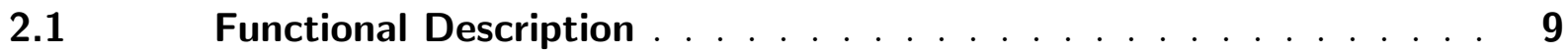

2.2 The Pneumatic Diaphragm Valves . . . . . . . . . . . 9

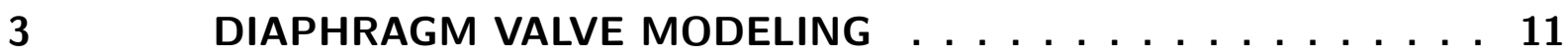

$3.1 \quad$ First Principle Diaphragm Valves Modeling . . . . . . . . . . 11

3.1.1 Friction Phenomenon . . . . . . . . . . . . . . . . . . 12

3.1.1.1 Presliding State . . . . . . . . . . . . . . . . . 13

$3.1 .1 .2 \quad$ Sliding state . . . . . . . . . . . . . . . . . . . 15

$3.1 .2 \quad$ Friction Models . . . . . . . . . . . . . . . . . . . 16

3.1.2.1 The Karnopp Model . . . . . . . . . . . . . . . . . . . . . 16

3.1.2.2 The LuGre Model . . . . . . . . . . . . . . . . . . . . . . . . 17

3.1.2.3 The GMS Model . . . . . . . . . . . . . . . . . . . . . . . . . . 17

3.1.2.4 The Dwell Time . . . . . . . . . . . . . . . . . . . . . . 18

3.2 Diaphragm Valve Model Discretization . . . . . . . . . . 19

3.2.1 Valve Discrete Model . . . . . . . . . . . . . . . . . . . . . . . . . . 19

3.2.2 Karnopp Discrete Model . . . . . . . . . . . . . . . . . . . . 20

3.2.3 LuGre Discrete Model . . . . . . . . . . . . . . . . . . . . . . 20

3.2.4 GMS Discrete Model . . . . . . . . . . . . . . . . . . . . . 21

3.2 .5 Sampling Time Selection . . . . . . . . . . . . . . . . 21

3.3 Data Driven Model of Pneumatic Control Valves . . . . . . . . . . 22

3.4 Diaphragm Valve Simulation . . . . . . . . . . . . . . . 24

4 OPEN LOOP PARAMETERS ESTIMATION . . . . . . . . . 28

$4.1 \quad$ A Priori Estimation of Parameters . . . . . . . . . . . . . 29

$4.2 \quad$ Open Loop Friction Parameters Estimation . . . . . . . . . . . . 34

4.2.1 Kano Search Space Analysis . . . . . . . . . . . . . . . . . 36

4.2.2 Karnopp Search Space Analysis . . . . . . . . . . . . . . 36 
4.2.3 LuGre Search Space Analysis . . . . . . . . . . . . . . . . . . . . . . . 41

$4.2 .4 \quad$ GMS Search Space Analysis . . . . . . . . . . . . . . . . . . 43

4.2.5 Optimization Method Selection . . . . . . . . . . . . . . . 45

4.2.5.1 Genetic Algorithm Optimization (GA) . . . . . . . . . . . . . . 46

4.2.5.2 Differential Evolution Optimization (DE) . . . . . . . . . . . . 47

4.2.5.3 Monte Carlo with Simplex Optimization (MS) . . . . . . . . . . . . . . 48

4.2.5.4 Successive Nonlinear Linear Regression (SNL) . . . . . . . . . . . . . . 48

4.2.5.5 Grid Search with Differential Evolution and Simplex Optimization (GDES) . . . 49

$4.3 \quad$ Simulated Tests . . . . . . . . . . . . . . . . 51

$4.4 \quad$ Experimental Tests . . . . . . . . . . . . . . . 56

4.4.1 Graphite Gasket Model Prediction Analysis . . . . . . . . . . . . . . . 62

4.4.2 Teflon Gasket Model Prediction Analysis . . . . . . . . . . . . . . . . 70

5 CLOSED LOOP PARAMETER ESTIMATION . . . . . . . . . . 78

$5.1 \quad$ Valve Diagnostics and Friction Detection . . . . . . . . . . . 78

$5.2 \quad$ Closed Loop Parameter Estimation . . . . . . . . . . . . . . . . 84

$5.3 \quad$ Stochastic Parameters Estimation Analysis . . . . . . . . . . . . 85

5.3.1 Karnopp Friction Model . . . . . . . . . . . . . . . 85

$5.3 .2 \quad$ LuGre Friction Model . . . . . . . . . . . . . . . . . . . . 87

$5.3 .3 \quad$ GMS Friction Model . . . . . . . . . . . . . . . . . . . . 89

$5.3 .4 \quad K a n o$ Valve Model . . . . . . . . . . . . . . . . . . . . . 91

5.3.5 A Generalized Valve Model . . . . . . . . . . . . . . . . . . . . . . 91

5.3.6 Open Loop Parameter Estimation Analysis . . . . . . . . . . . . . . . . . . 91

5.3.7 Closed Loop Parameter Estimation Analysis . . . . . . . . . . . . . . . . . 93

$5.4 \quad$ Simulated Tests . . . . . . . . . . . . . . . . . . 94

$5.5 \quad$ Experimental Tests . . . . . . . . . . . . . . . . 98

5.5.1 Graphite Gasket Model Prediction Analysis . . . . . . . . . . . . . . . 102

5.5.2 Teflon Gasket Model Prediction Analysis . . . . . . . . . . . . . . . . . 108

$5.6 \quad$ Valve Model Selection . . . . . . . . . . . . . . . 115

6 AUTOMATIC FRICTION COMPENSATION . . . . . . . . . 117

6.1 The AIC framework . . . . . . . . . . . . . . 118

$6.2 \quad$ A First Test with PID control . . . . . . . . . . . . 120

$6.3 \quad$ A Robustness Test . . . . . . . . . . . . . . . . 125

6.3.1 The Control Freezing Algorithm (CF) . . . . . . . . . . . . 126

6.3.2 The Knocker Compensator . . . . . . . . . . . . . . . . 126

6.3.3 The Constant Reinforcement Compensator (CR) . . . . . . . . . . . 127

6.3.4 The Fuzzy PID Controller . . . . . . . . . . . . . . . . . . . 127

6.3.5 Robustness Test Results . . . . . . . . . . . . . . 128 
6.4 Test of the Sliding Mode Control and of the Feedforward PID with Integral Deadband Structure . . . . . . . . . . . . . . . . . . 135

6.4.1 The Integrative Sliding Mode Control (ISMC) . . . . . . . . . . . 135

6.4.2 The Feedforward PID with Integral Deadband (FFPID) Controller . . . . . 137

$6.4 .3 \quad$ Test Results . . . . . . . . . . . . . . . . . . . . . . . 138

6.5 Test with the Teflon Gasket Valve . . . . . . . . . . . . . 148

6.6 Concluding Remarks . . . . . . . . . . . . . . . . 152

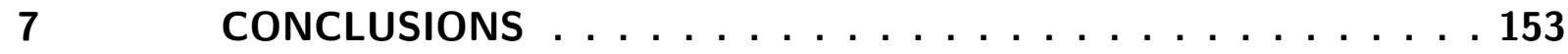

REFERENCES . . . . . . . . . . . . . . . 155

$\begin{array}{ll}\text { APPENDICES } & 159\end{array}$

APPENDIX A-OPTIMIZATION ALGORITHMS . . . . . . 160 


\section{Introduction}

Pneumatic diaphragm valves are extensively used in the process industries to regulate flow (GARCIA, 2008). The valve performance is important to guarantee control loops stability, compensate process perturbations and maintain production quality. Evidently, the valves are designed to reach a high reliability, as most industries tend to work uninterruptedly for several months. However, defects may appear due to wear or an external event, reducing considerably the production quality.

The pneumatic diaphragm valve is essentially a servomechanism. It transforms the air pressure into a stem movement using a diaphragm and a mechanical system. For each stem position there is a corresponding pressure drop across the valve, which is used to modify the process flow rate. A very frequent defect in pneumatic diaphragm valves is a friction increase in the stem packing, which can severely affect the system precision, as is the case for any other servomechanism (ARMSTRONG-HÉLOUVRY; DUPONT; CANUDAS de WIT, 1994). Regarding diaphragm valves, this friction increase results in oscillatory control loops, which can potentially affect many coupled control loops. In fact, defective valves accounts for a fair proportion of suboptimal performing control loops in industry (CHOUDHURY et al., 2006; THORNHILL; HORCH, 2007).

If the control loop oscillations are tolerable, the plant manager may decide to endure the consequences of this defective valve (e.g. production drop) rather than stop the system to perform maintenance. This scenario motivated the design of friction compensation algorithms, implemented in the control system, that are able to improve the performance of such defective valves. Inevitably, these algorithms rely on estimating parameters of a given friction model for then tuning the friction compensation algorithm (SILVA; GARCIA, 2014; HIDALGO; GARCIA, 2017).

Modeling friction, however, is often a difficult task, as the models are usually nonlinear and discontinuous. Yet, friction modeling is a research area that evolves with higher precision instruments and experiment design. Therefore, many friction models were suggested by researchers, as older models were not able to predict novel experimental data. With respect to pneumatic diaphragm valves, the vast majority of researchers focused on working with the most frequently encountered valves in industries, which are the simplest ones, with open loop stem position control and no embedded sensors. Naturally, the friction models selected to simulate such simple valves were also the simplest ones. However, valve suppliers are starting to use embedded digital systems and sensors in new pneumatic diaphragm valves, in order to increase their performance and reliability. Therefore, in such valves, it is possible to apply state of the art friction models and compensation algorithms. 
This thesis aims to provide an algorithm, to be embedded in a digital positioner, that is able to detect, estimate and compensate friction in pneumatic diaphragm valves with dedicated sensors. Necessarily, to estimate friction, it is imperative to select a model. In this work, two friction models, which were vastly explored in the diaphragm valves modeling area are compared to two relatively new friction models, which rarely or were never used at all for modeling diaphragm valves.

The automatic friction compensation is developed by using the Adaptive Inverse Control (AIC) technique, which is a branch of adaptive indirect control method, that relies on estimating the plant parameters for then tuning the controller parameters (ASTRÖM; WITTENMARK, 1995; WIDROW; WALACH, 2008).

\subsection{Motivation}

The friction compensation techniques are usually difficult to be applied in practice, since they demand knowledge in estimating friction and in implementing the compensation in the control system. The development of an algorithm that is able to maintain the valve performance, regardless of friction deviations and with minimal external influence brings the research developments closer to the industrial applications.

The diaphragm valves modeling and friction compensation techniques are strongly based on friction models that are outdated with the state of the art related to friction models proposed by tribology science. This has attracted criticism from the scientific community and therefore demands analysis and testings of these models.

The AIC framework is able to deliver optimal controllers for nonlinear and discontinuous plants, regardless of the controller structure. Consequently, some empirical or practical rules used to tune friction compensators can be improved.

\subsection{Objectives}

The objective of this thesis is to deliver a framework to detect and estimate friction deviations of a pneumatic diaphragm valve and automatically tune the stem position controller to compensate such friction fluctuation. To achieve this overall objective, the following intermediate objectives are necessary:

i. Study the friction characteristics, its models and select the most reasonable model;

ii. Design a method to estimate the valve and friction parameters in open and closed loop systems;

iii. Design the stem position controller and friction compensation, based on the AIC framework; 
iv. Perform proof of concept tests of the proposed methods using the Flow Pilot Plant (FPP) of the Industrial Process Control Laboratory (IPCL).

\subsection{Literature Review}

This section presents some of the most relevant works for this thesis development.

With regard to pneumatic diaphragm valves modeling, there are two model structure choices: the first principle and the data driven models. The latter are the simpler models, simplifying many valve and friction characteristics to obtain a fair simulation of a valve with given friction index. The first principle modeling uses well known physical laws to create the valve model. One unknown variable in these models is the friction force, which has to be calculated using one of the available friction models.

The most relevant pneumatic diaphragm valves data driven models, that are broadly used for modeling and compensation of diaphragm valves are the Kano and Choudhury models (KANO et al., 2004; CHOUDHURY; THORNHILL; SHAH, 2005). The Kano model is capable of predicting the expected behavior of valve signature curves, which are used for valve diagnostics (GARCIA, 2008). These models are frequently used to model valves with no embedded sensors and position controllers, as it would be difficult to estimate the parameters of a more complex valve model without proper sensors.

Modeling pneumatic control valves using a first principle modeling technique is straightforward, as shown by Kayihan and Doyle III (2000), Garcia (2008). The friction model, though, is the most complex part of the first principle valve model.

Many friction models were proposed by researchers and the greater reason for that is because older models failed to predict new experimental data, as the sensor precision and experiment design improved. Other friction models were proposed to ease the simulation complexity and mathematical analysis, as the most complex models are discontinuous and nonlinear. Sometimes, simpler friction models are selected to permit mathematical analysis, at the expense of a poorer friction prediction. Karnopp (1985) proposed a method to simulate the transition from static to dynamic friction using the Stribeck curve. This model is frequently used for modeling pneumatic diaphragm valves. Armstrong-Hélouvry, Dupont and Canudas de Wit (1994) reviewed the tribology literature, exposing relevant friction aspects and models and eventually suggesting a new model named Seven Parameter Friction Model, which is able to predict many friction characteristics that can be observed experimentally. Canudas de Wit et al. (1995) proposed the Leuven-Grenoble (LuGre) model, aiming to deliver a fair prediction of the most important friction dynamics in presliding and sliding states while maintaining a rather simple mathematical structure in order to favor control system design and analysis. This model was only tested for simulation of pneumatic diaphragm valves Garcia (2008), but was never used for friction compensation. 
The LuGre model, although efficient and useful for friction compensation, was proven imprecise for presliding state friction prediction. To correct this inaccuracy, Swevers et al. (2000) proposed the Leuven friction model but, as it was considered complex for simulation and analysis, Al-Bender, Lampaert and Swevers (2005) suggested a generalization of the Maxwell-Slip (GMS) model that was able to fix the LuGre model flaw while using simple mathematical functions. In this thesis, the Karnopp, LuGre and GMS models are used for simulating pneumatic diaphragm valves.

The estimation of the friction parameters of data driven pneumatic diaphragm valve models is studied by many authors. Some propose analysis of the valve signature curve, using the stem position and controller output, which is pretty straightforward (KANO et al., 2004; CHOUDHURY; JAIN; SHAH, 2008). Other methods are more complex, as they assume that the stem position is not available and deliver the friction parameters by analyzing the oscillatory behavior of the controller output and process variable (KANO et al., 2004; CHOUDHURY et al., 2006). Jelali (2008) proposed an algorithm to estimate the friction parameters, in closed loop systems, using a Hammerstein model, where the Kano model was considered as the nonlinear input function, using only the controller output and process variable. The Hammerstein model parameters, and consequently the Kano model parameters, were estimated using a genetic algorithm for the nonlinear model and a linear estimator for the dynamic model. Romano and Garcia (2011) expanded the work of Jelali (2008), by estimating the parameters of a Hammerstein-Wiener model, composed by the Kano model as the nonlinear model input function, followed by a dynamic linear system and then a static cubic splines function. The parameters were estimated using grid search, simplex and quadratic programming. Additionally, Romano and Garcia (2011) used a specific residual function to avoid parameter bias due to the correlation between input and process noise when estimating Wiener models.

The estimation of valve and friction parameters using first principle valve modeling and Karnopp friction model was discussed by Garcia (2007), Romano and Garcia (2007), Romano and Garcia (2008), who proposed force balance equation analysis and linear regression methods.

Other than pneumatic diaphragm valves, friction parameters estimation is a widely explored research area. For the simpler models, authors usually suggest linear estimator techniques, as the least squares estimator (ARMSTRONG-HÉLOUVRY; DUPONT; CANUDAS de WIT, 1994). As the friction model gets more complex, the use of derivative free optimization methods, either alone or combined with a linear estimator, is commonplace (CANUDAS de WIT; LISCHINSKY, 1997; SWEVERS et al., 2000; TJAHJOWIDODO; BRUSSEL, 2005; AL-BENDER; LAMPAERT; SWEVERS, 2005; WORDEN et al., 2007; RIZOS; FASSOIS, 2009). Usually, researchers suggest that the excitation signal design need to evidence all friction phenomena for a correct parameter estimation. The methods they propose, however, are based on indirect but precise friction force measurement, which 
is unavailable when dealing with pneumatic diaphragm valves.

As for friction compensation, many researchers have proposed techniques for a wide range of systems. Regardless of the knowledge area, researchers often suggest using high frequency signals, added to the system controller, to overcome the static friction (CANUDAS de WIT et al., 1991; HÄGGLUND, 2002; SRINIVASAN; RENGASWAMY, 2008; CUADROS; MUNARO; MUNARETO, 2012; SILVA; GARCIA, 2014). Feedback linearization and sliding mode control are also common methods for servomechanisms and pneumatic diaphragm valves (CANUDAS de WIT; LISCHINSKY, 1997; KAYIHAN; DOYLE III, 2000; FREIDOVICH et al., 2010; BAEZA; GARCIA, 2018). Hidalgo and Garcia (2017) designed a sliding mode control with feedback linearization and friction observer using the Karnopp friction model, for valves with digital positioners.

With respect to diaphragm valves friction compensation, some authors suggest that the backlash compensation method can be used to overcome static friction as well, resulting in the constant reinforcement compensator (IVAN; LAKSHMINARAYANAN, 2009). Retuning the controller or using an adaptive integral term is often proposed by authors, since the controller integral term plays a major role in inducing limit-cycles in control loops (MOHAMMAD; HUANG, 2012; MISHRA; KUMAR; RANA, 2014).

Regarding motor operated servomechanisms, many authors suggest adaptive friction compensation due to its easier implementation: it eliminates the need to know any parameters beforehand and may be able to compensate for time-varying characteristics (FEEMSTER et al., 1999; MÁRTON; LANTOS, 2007; SOBCZYK et al., 2016; VERBERT; TÓTH; BABUSKA, 2016). Adaptive friction compensation, however, result in complex nonlinear equations and to prove the closed loop system stability, in some cases it is necessary to simplify the friction model. Yet, some authors suggest using feedforward compensation, by calculating the friction force given the system set point (SWEVERS et al., 2000; TJAHJOWIDODO; BRUSSEL, 2005).

\subsection{Contributions}

Some of the new friction models proposed by the tribology science researchers have never been tested for diaphragm valves modeling. The main reason for that is because the friction compensation area focused on delivering solutions for the already installed valves, which frequently lack sensors and therefore would hardly benefit from new models. However, this assumption was never tested and analyzed properly.

Estimating friction parameters is usually a difficult task, due to its nonlinear and discontinuous nature. It is an even more complex task with regard to diaphragm valves as the friction force is not measured, its parameters have to be estimated indirectly from stem position and diaphragm pressure measurements. No other work has focused on 
estimating friction parameters of novel models, for diaphragm valves. In this work the GMS model is used to describe the effect of friction in diaphragm control valves, as seen in Subsection 3.2.4, something that was never made before, therefore constituting an important contribution of this thesis. An enhancement in the Karnopp friction model, that is frequently used for modeling pneumatic control valves, is also proposed in this work, as can be seen in Subsection 3.2.2.

Diaphragm valves friction compensation using the AIC framework has never been developed. This method is rarely used for control systems, but has the potential to improve the tuning of already developed friction compensation techniques and to be used to solve many other control problems.

\subsection{Publications}

The question that arises when dealing with nonlinear and discontinuous systems parameter estimation is whether a linear objective function optimization technique would be enough for parameters estimation. This problem was addressed and published in the annals of the 12th international IFAC Symposium on Dynamics and Control of Process Systems, Including Biosystems, whose title is Stribeck Parameters Estimation of a Diaphragm Valve Using Quasi Newton Method. In this paper, analytical derivatives of the valve with Karnopp friction model were derived and the Quasi Newton method was used to estimate the friction parameters. The results, however, were not promising, due to the high mathematical complexity of the method and numerical problems, but it is enough to support the use of derivative free optimization methods for friction parameters estimation.

The diaphragm valve modeling and parameters estimation was transformed into a paper and was sent to a control systems journal.

The software and experimental data of this work are stored in Zenodo (MARQUES, 2019).

\subsection{Thesis Outline}

The objective of Chapter 2 is to introduce the laboratory used to obtain the proof of concept tests. The instruments, equipment and materials used to obtain the experimental data are presented.

Modeling diaphragm valves with continuous and discrete equations is presented in Chapter 3.

The valve model parameters estimation in open loop systems is developed in Chapter 4, whereas the closed loop systems estimation method is designed in Chapter 5. 
The automatic compensation of the valve friction is developed in Chapter 6, using the Adaptive Inverse Control framework.

The conclusions and final remarks of this work are presented in Chapter 7 .

Appendix A presents the optimization algorithms used in this thesis. 


\section{The Flow Pilot Plant}

The Flow Pilot Plant (FPP) is located at the Industrial Process Control Laboratory (ICPL) of the Escola Politécnica of the University of São Paulo (EPUSP) and it is used for acquiring the experimental data of this thesis. Its simplified Piping \& Instrumentation Diagram (P\&ID) is presented in Figure 2.1, where the auxiliary instruments and equipment were omitted. The FPP uses industrial tier equipment and instruments, therefore it is possible to perform proof of concept tests that are pretty similar to what is normally found in industry.

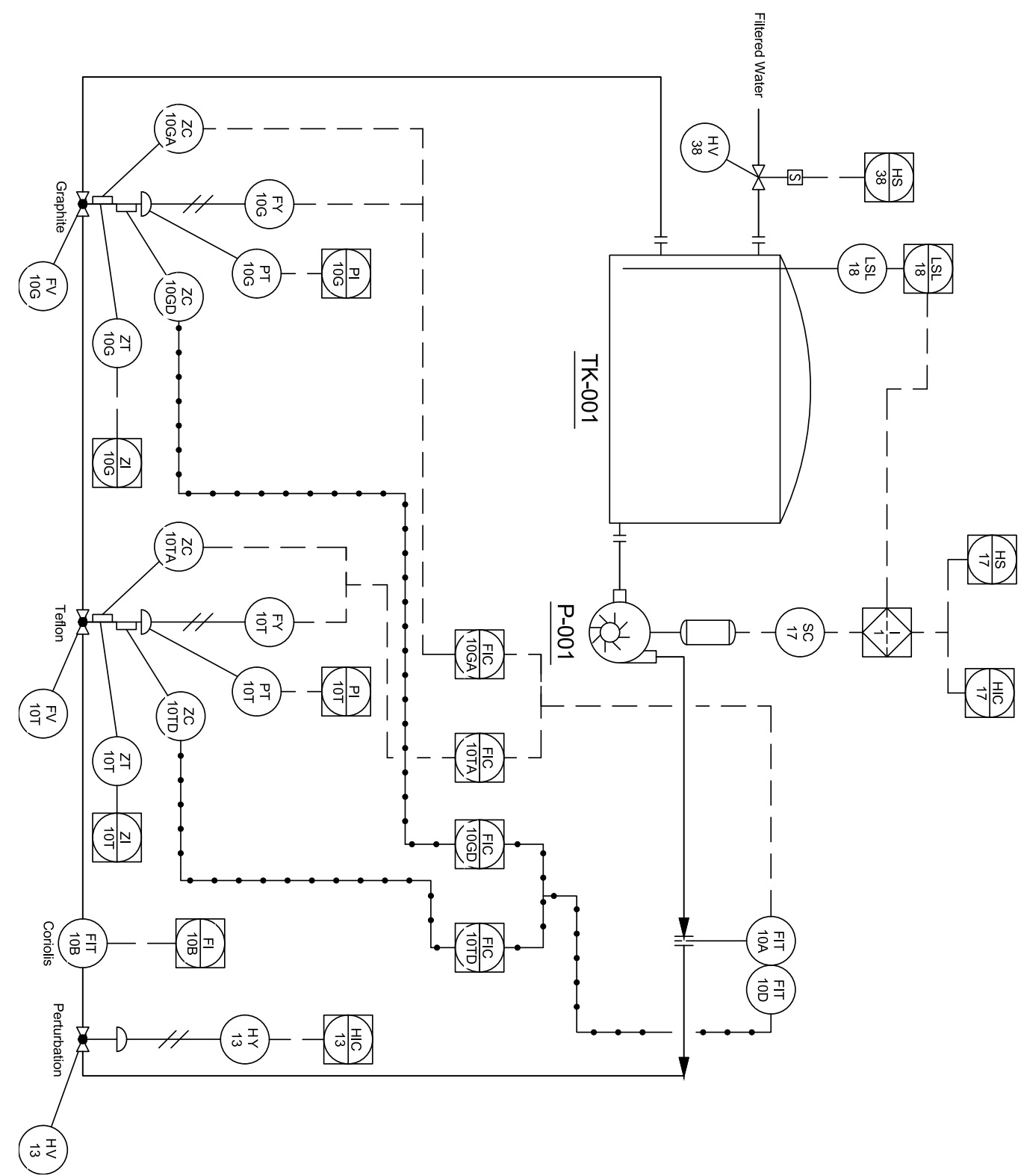

Figure 2.1 - Simplified P\&ID of the FPP.

Source: Based on Mora (2014). 


\subsection{Functional Description}

The FPP is a closed loop hydraulic circuit that uses a pump to increase the fluid pressure and thus forces a flow through its pipes. The tank TK-001 stores filtered water, accommodates density variations and guarantees the minimum suction pressure of the pump $\mathrm{P}-001$, in order to avoid cavitation. If TK-01 water level is low (LSL-18), the interlock $\mathrm{I}-1$ turns off $\mathrm{P}-001$. The pump has a velocity regulator ( $\mathrm{SC}-17)$, which is able to control the pump discharge pressure.

The hydraulic circuit flow rate is measured using an orifice plate (FE-10) and differential pressure measurements (FIT-10A/10D) or using a Coriolis sensor (FIT-10B). The flow can be controlled using a teflon (FV-10T) or graphite (FV-10G) gasket pneumatic diaphragm valve. The teflon gasket usually presents lower friction index than the graphite, but the latter is preferable when controlling high temperature fluids. Therefore, it is possible to test real valves with different friction characteristics. The valve FV-13 is used to cause control loop perturbations.

The control system can be implemented in an $\mathrm{ABB}^{\circledR}$ model $800 \mathrm{xA}$ Digital Control System (DCS), or using the PCI-6229 module from National Instruments ${ }^{\circledR}$ and a real-time computer software.

\subsection{The Pneumatic Diaphragm Valves}

The pneumatic diaphragm valves $(\mathrm{FV}-10 \mathrm{~T} / 10 \mathrm{G})$ are manufactured by Fisher and are air-to-close, with globe plug. The diaphragm pressure (PT-10T/10G) and the stem position (ZT-10T/10G) are measured variables. A Linear Variable Differential Transformer (LVDT) is used for measuring the stem position.

In this thesis, the diaphragm pressure is controlled by using a current to pressure converter (I/P converter FY-10T/10G), which converts a $4-20 \mathrm{~mA}$ DC current to a 6 30 psi pressure. Even though it is not used in this thesis, there is the possibility to use an electro-pneumatic or a digital positioner. The former has a mechanical feedback of the valve stem position to adjust the diaphragm pressure and the latter uses an embedded digital system to adjust the diaphragm pressure given a deviation from desired and measured stem position.

Both valves have the same diaphragm actuator (Fisher 657) which has a cross sectional area of $S_{a}=445 \cdot 10^{-4} \mathrm{~m}^{2}$ and works from 6 to 30 psi (EMERSON, 2000). Therefore, from the valve manual, the minimum pressure is $P_{\min }=41368.5 \mathrm{~Pa}$ and considering that the controller output is normalized from $0-100 \%$, the I/P converter pressure gain is $K_{P_{g}}=1654.75 \mathrm{~Pa} / \%$. The valve moving parts mass is $m=1.6 \mathrm{~kg}$, as informed by the vendor. The minimum and maximum stem position physical constraints 
are $x_{\min }=0 \mathrm{~m}$ and $x_{\max }=28.58 \cdot 10^{-3} \mathrm{~m}$, respectively.

The experimental data presented in this thesis are obtained using the MATLAB ${ }^{\circledR}$ Real-Time toolbox and the PCI-6229 hardware interface.

In this thesis, the diaphragm pressure and stem position are assumed to be available to the digital positioner, in which the algorithms developed in this work are to be embedded. It is not common to measure the diaphragm pressure in digital positioners, but as the sensors are constantly getting cheaper with the internet of things development, the added sensor is worth the cost, as the valve performance can be improved even when there are deviations from its design parameters and yet, it allows the development of better defect detection algorithms. 


\section{Diaphragm Valve Modeling}

The diaphragm valve modeling is addressed in this chapter. It starts by presenting how pneumatic diaphragm valves are modeled using the first principles method. As this modeling requires a friction model, the observed experimental friction characteristics and the Karnopp, LuGre and GMS friction models are presented. Yet, the Kano data driven diaphragm friction valve model is also presented. The models are discretized to be used in further developments.

\subsection{First Principle Diaphragm Valves Modeling}

The diaphragm control valves operate by the interaction of electro-pneumatic, mechanical and hydraulic systems. Figure 3.1 presents a simplified schematic air to open diaphragm valve.

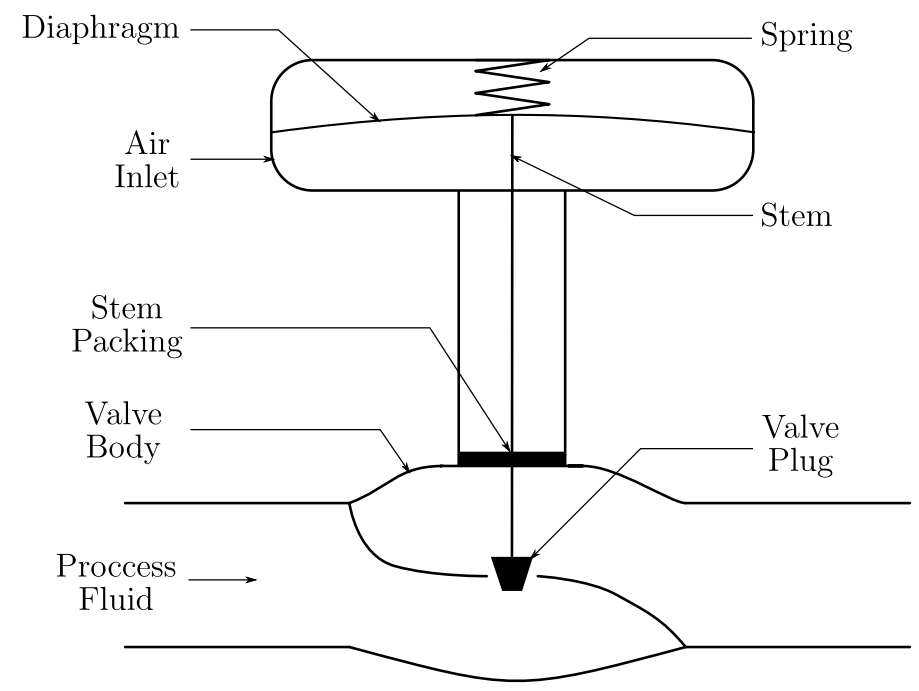

Figure 3.1 - Simplified schematic representation of a pneumatic diaphragm valve. Source: Based on Emerson (2005).

As the pressure varies, the diaphragm converts the pressure into a force, which accelerates the mechanical system, moving the stem until a new stable point is reached due to spring compression or extension. As the stem position changes, the pressure drop across the valve is modified, which in turn modifies the hydraulic system flow rate.

The first principles modeling is straightforward, by applying force balance in the simplified pneumatic and mechanical systems shown in Figure 3.1, one obtains (KAYIHAN; DOYLE III, 2000; GARCIA, 2008):

$$
m \ddot{x}(t)=F_{\text {diaphragm }}(t)-F_{\text {spring }}(t)-F_{f}(t)-F_{\text {flow }}(t)-F_{\text {init }},
$$


where $x$ is the stem position, $m$ is the moving parts mass, $F_{\text {diaphragm }}$ is the diaphragm force, $F_{\text {spring }}$ is the spring force, $F_{f}$ is the friction force, $F_{\text {flow }}$ is the force the fluid exerts on the valve plug due the flow and $F_{\text {init }}$ is the spring initial force, when the system is at rest. The diaphragm force is:

$$
F_{\text {diaphragm }}(t)=S_{a} P(t)
$$

where $S_{a}$ is the diaphragm section area and $P(t)$ is the air pressure in the valve diaphragm chamber. The spring force can be modeled as:

$$
F_{\text {spring }}(t)=k x(t)
$$

where $k$ is the spring constant. $F_{\text {flow }}$ can be neglected, since it is much lower than the other forces (KAYIHAN; DOYLE III, 2000).

The I/P converter has nonlinear dynamics, presenting different characteristics given the controller output (OP) magnitude, direction and signal characteristics (MARQUES; GARCIA, 2019). As for filtering and estimating the valve bandwidth, since the diaphragm pressure is considered a measured variable in this thesis, a first order filter suffices to model the diaphragm pressure:

$$
\dot{P}(t)=\frac{\left(K_{P_{g}} \mathrm{OP}(t)+P_{\mathrm{min}}\right)-P(t)}{\tau_{\mathrm{I} / \mathrm{P}}},
$$

where OP is the controller output, normalized to $0-100 \%, K_{P_{g}}$ is a pressure gain, $P_{\min }$ is the pressure when $\mathrm{OP}$ is zero and $\tau_{\mathrm{I} / \mathrm{P}}$ is the $\mathrm{I} / \mathrm{P}$ model time constant. The pressure gain is defined as:

$$
K_{P_{g}}=\frac{P_{\max }-P_{\min }}{100}
$$

where $P_{\max }$ is the maximum diaphragm pressure.

With all terms defined but the friction force, $F_{f}$, the friction characteristics are discussed and the Karnopp, LuGre and GMS friction models are presented in the following sections.

\subsubsection{Friction Phenomenon}

Friction is a phenomenon that has been studied since Leonardo da Vinci, however, due to its complexity, there is not a universal friction model that would be suitable for all applications. In practice, every friction model has a foundation in experimental data (is a data driven model) and is created to encompass some objectives, such as ease of mathematical analysis, ease of implementation, prediction precision and physical consonance. Understanding friction is imperative for selecting a proper model. 
The friction force, $F_{f}$, arises from the microscopic interactions between two surfaces, as presented in Figure 3.2, which makes it practically impossible to create an extensive model, which considers every microscopic interaction between the material surfaces. In practice, the friction models intend to capture one or more macroscopic behaviors observed experimentally. Often, friction is classified into two states with different characteristics: presliding and sliding.

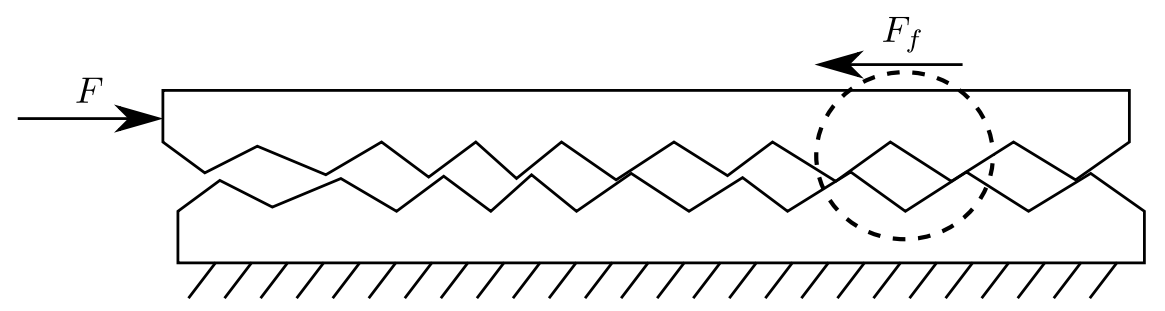

Figure 3.2 - Friction origin.

Source: Based on Olsson (1996).

\subsubsection{Presliding State}

In the presliding state, the surfaces remain sticking until the external force $(F)$ is higher than the static friction force or stiction $\left(F_{s}\right)$. Some models assume that there is no motion between the surfaces until $F>F_{s}$. However, experimental data has proven that there is a relative motion between the surfaces, proportional to the external force, which is often explained as the elastic deformation between the surfaces irregularities as shows the Figure 3.3 depiction.
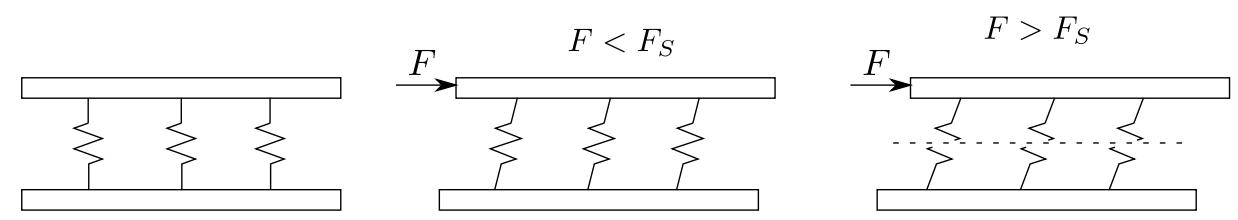

Figure 3.3 - Depiction of presliding state.

Source: Based on Olsson (1996).

This motion is of the order of a few micrometers, but can be amplified by the mechanical system to an order of a few millimeters (ARMSTRONG-HÉLOUVRY; DUPONT; CANUDAS de WIT, 1994). Additionally, the experimental findings suggest that the presliding regime can be described by a hysteresis function with nonlocal memory (SWEVERS et al., 2000). Figure 3.4 summarizes the previous statement, which is known as presliding displacement. Note that there is a remnant displacement when the external force is removed, but, this does not imply that it is possible to reach any displacement by applying and removing a force lower than $F_{s}$.

Another presliding characteristic that is common to observe experimentally is the inverse relationship between the external force rate of change and the breakaway force $\left(F_{s}\right)$. This behavior is shown qualitatively in Figure 3.5. 


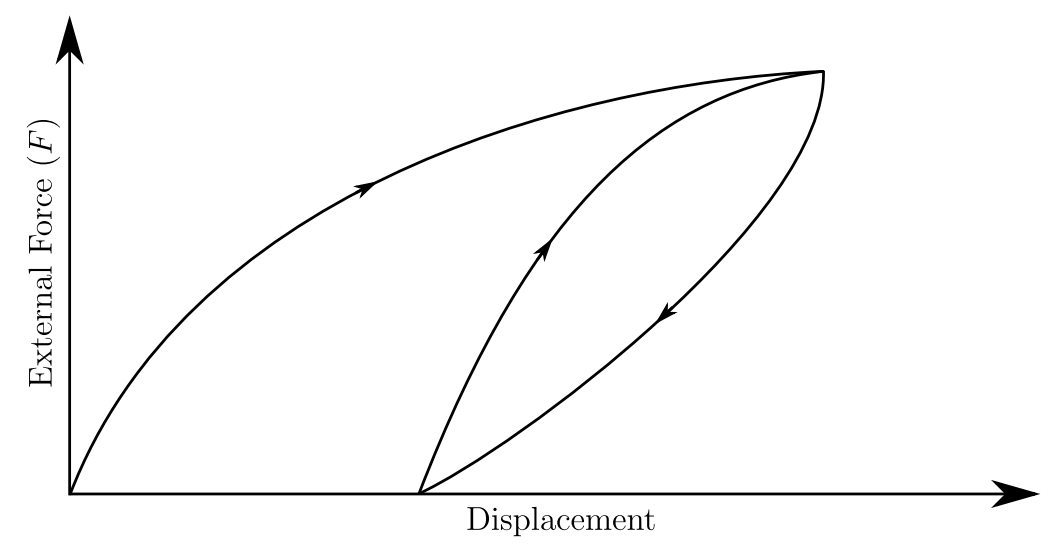

Figure 3.4 - Presliding displacement.

Source: Based on Olsson et al. (1998), Swevers et al. (2000).

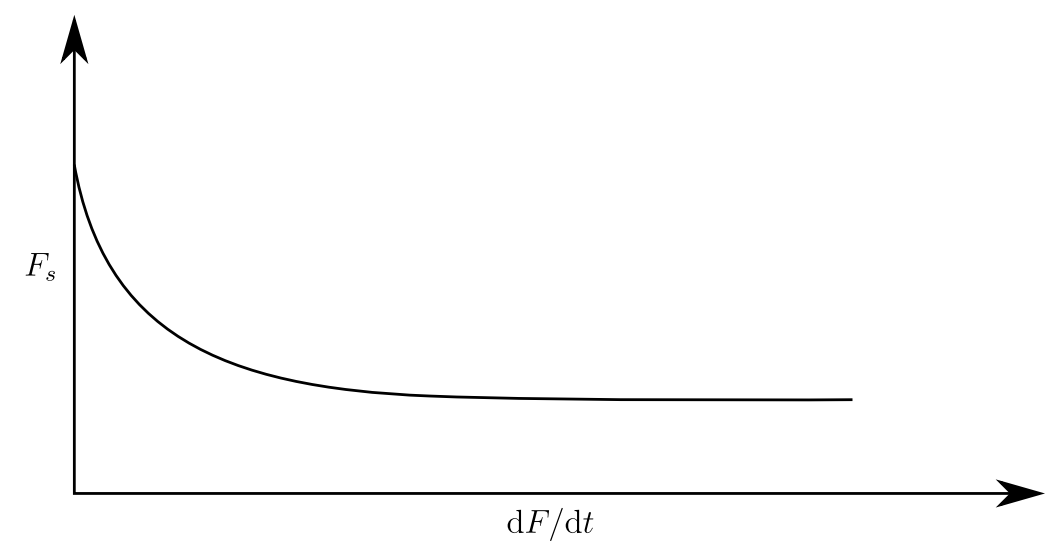

Figure 3.5 - Decrease of $F_{s}$ with an increase in the rate of change of the external force $F$.

Source: Based on Olsson et al. (1998).

Yet, $F_{s}$ increases with the amount of time the system has spent sticking. The increased magnitude and the times involved depend on the materials and lubricant used in the mechanical system. This relationship is shown in Figure 3.6.

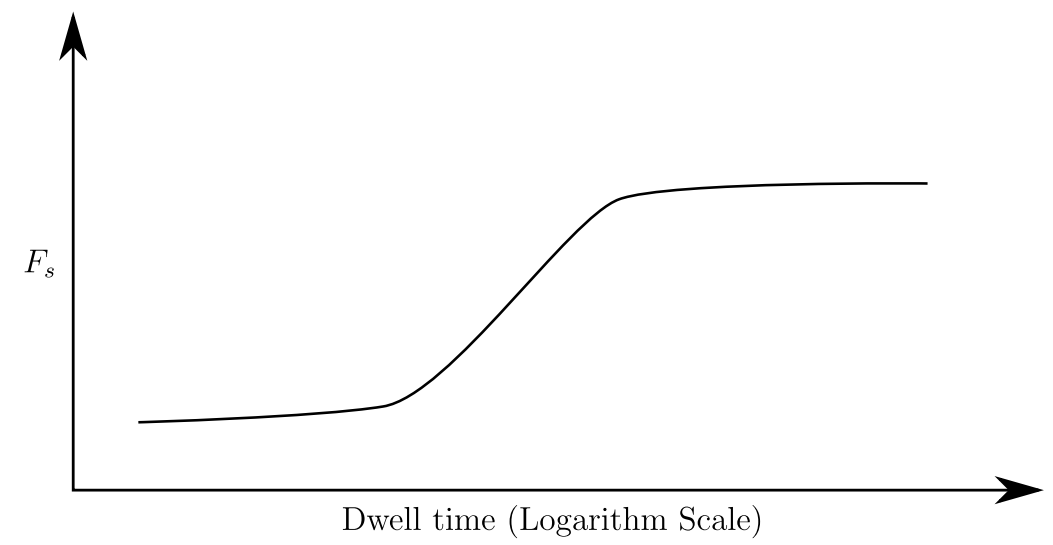

Figure 3.6 - The relationship between $F_{s}$ and the dwell time.

Source: Based on Armstrong-Hélouvry, Dupont and Canudas de Wit (1994). 


\subsubsection{Sliding state}

The most known characteristic of the sliding state is a friction-velocity steady state relationship, which can be modeled by a combination of three effects that are function of the materials, lubricant and normal force. The first is the Coulomb friction force $\left(F_{c}\right)$ that is modeled as a constant force, opposed to the movement direction, as long as the relative velocity is different from zero. The second is a directly proportional relationship between velocity and the friction force, which is modeled by the viscous friction parameter $\left(F_{v}\right)$. The last behavior corresponds to the transition from presliding to sliding. Many functions can be used to model this transition, but in any case it is characterized by a constant, the Stribeck Velocity $\left(v_{s}\right)$, which dictates the velocity range in which $F_{s}$ converges to $F_{c}$. Figure 3.7 aggregates all the three behaviors. Note that, in general, $F_{s} \geq F_{c}$.

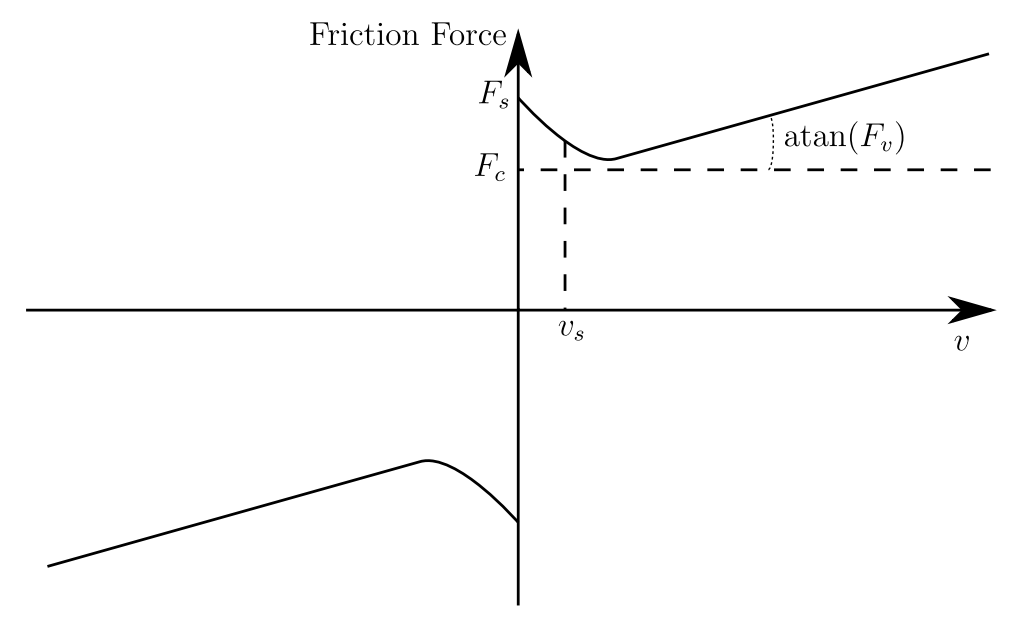

Figure 3.7 - The friction-velocity relationship in steady state condition. Source: Based on Armstrong-Hélouvry, Dupont and Canudas de Wit (1994).

Although Figure 3.7 suggests an apparent instantaneous change in the friction force due a velocity variation, this is not what occurs in experimental observations. There is a lag between the velocity variation and its respective change in the friction force, which can be observed by varying the velocity sinusoidally, without reversing the movement direction. The friction force lag creates a hysteretic behavior, as shown in Figure 3.8. 


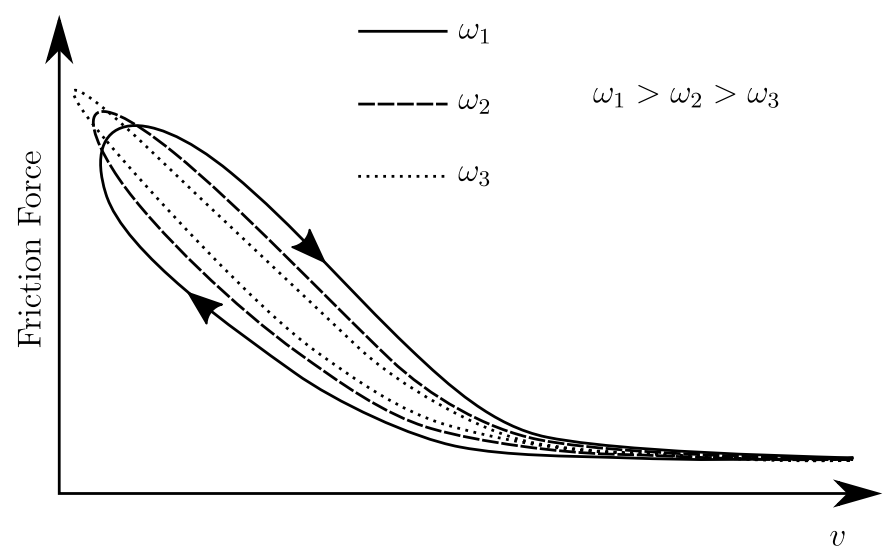

Figure 3.8 - Depiction of friction lag, perceived due to different oscillation frequencies.

Source: Based on Armstrong-Hélouvry, Dupont and Canudas de Wit (1994).

\subsubsection{Friction Models}

Each friction model tends to capture one or more friction characteristics. Naturally, a very precise model tends to be more complex and has more parameters than a model which is able to predict only a few characteristics. The Karnopp, LuGre and GMS models are presented in the following sections.

\subsubsection{The Karnopp Model}

The Karnopp model is useful when it is necessary to predict the friction-velocity in steady state (KARNOPP, 1985). This model disregards all presliding characteristics and the dynamic behaviors of the sliding regime. The friction force $\left(F_{f}\right)$ is calculated as:

$$
F_{f}= \begin{cases}F, & \text { if }|v|<D V \text { and }|F| \leq F_{s} \\ F_{s} \operatorname{sgn}(F), & \text { if }|v|<D V \text { and }|F|>F_{s} \\ F_{d}(v), & \text { if }|v| \geq D V\end{cases}
$$

where $F$ is the external force, $D V$ is a velocity that determines when the system is considered sticking, $v$ is the relative velocity of the $\operatorname{system,~} \operatorname{sgn}(x)$ is the signal function, defined as:

$$
\operatorname{sgn}(x)= \begin{cases}1, & \text { if } x>0 \\ 0, & \text { if } x=0 \\ -1, & \text { if } x<0\end{cases}
$$

and $F_{d}(v)$ is the steady state friction-velocity relation, also known as the Stribeck curve, which is commonly defined as:

$$
F_{d}(v)=\left[F_{c}+\left(F_{s}-F_{c}\right) \mathrm{e}^{-\left(v / v_{s}\right)^{2}}\right] \operatorname{sgn}(v)+F_{v} v
$$


Generally, to achieve better simulation results, when the valve is sticking - first case of Eq. (3.6) -, the system velocity is set to zero.

\subsubsection{The LuGre Model}

The LuGre model is designed to predict the presliding displacement, the decrease of $F_{s}$ with an $F$ rate of change increase, the friction-velocity relationship in steady state and the friction lag (CANUDAS de WIT et al., 1995). The model is described by the following equation:

$$
F_{f}(z, v)=\sigma_{0} z+\sigma_{1} \frac{\mathrm{d} z}{\mathrm{~d} t}+F_{v} v
$$

where $z$ is a virtual state regarded as the average deflection of the material surface bristles, $\sigma_{0}$ represents the elastic constant of the bristles and $\sigma_{1}$ is a damping coefficient. The variable $z$ is calculated as:

$$
\frac{\mathrm{d} z}{\mathrm{~d} t}=v-\frac{|v|}{g(v)} z
$$

where $g(v)$ is the friction-velocity function in steady state divided by $\sigma_{0}$, that can be defined similarly as Eq. (3.8):

$$
g(v)=\frac{F_{c}+\left(F_{s}-F_{c}\right) \mathrm{e}^{-\left(v / v_{s}\right)^{2}}}{\sigma_{0}} .
$$

In steady state, $z \rightarrow g(v) \operatorname{sgn}(v)$ and the average bristle deflection can be seen as a velocity filter with $g(v) /|v|$ time constant and variable gain.

\subsubsection{The GMS Model}

The LuGre model was proven to be imprecise in the predictions regarding the presliding displacement, as it can drift when in presliding state and excited with an external force lower than breakaway force (SWEVERS et al., 2000). The Generalized Maxwell Slip Model (GMS) was developed to encompass a precise simulation of the presliding displacement and ease of implementation (AL-BENDER; LAMPAERT; SWEVERS, 2005), besides incorporating all friction characteristics that the LuGre model was already able to predict. The GMS model consists of $N_{M e}$ virtual massless elements connected in parallel that, when the individual effects are added, results in the macroscopic friction effect, as shown in Figure 3.9.

The friction force is calculated as (PIATKOWSKI, 2014):

$$
F_{f}=\sum_{i=1}^{N_{M e}} F_{i}+F_{v} v,
$$

where $F_{i}=\kappa_{i} \psi_{i}+\nu_{i} \dot{\psi}_{i}$ is the friction force of element $i, \psi \in \mathbb{R}^{N_{M e}}$ represents the displacement of the virtual elements, $\kappa_{i}$ is the elastic constant of the element $i$ and $\nu_{i}$ is 


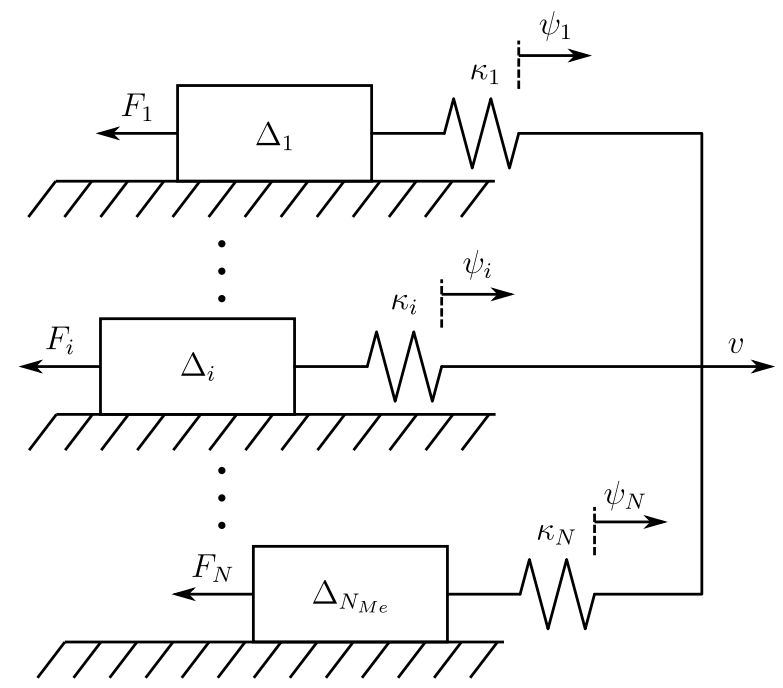

Figure 3.9 - The virtual massless elements of the GMS model.

Source: Based on Piatkowski (2014).

the damping coefficient of element $i$. When the element $i$ is in the presliding regime, $\psi_{i}$ is equal to:

$$
\dot{\psi}_{i}=v
$$

and the element remains in this state until $F_{i}>\alpha_{i} s(v)$, where $s(v)$ is the friction-velocity function:

$$
s(v)=F_{c}+\left(F_{s}-F_{c}\right) \mathrm{e}^{-\left(v / v_{s}\right)^{2}}
$$

and $\alpha_{i}$ is a constant, such that $\left\{\alpha_{i} \mid \sum_{i=1}^{N_{M e}} \alpha_{i}=1\right\}$. If the element $i$ is in the sliding regime, $\psi_{i}$ is calculated as:

$$
\dot{\psi}_{i}=\frac{C \alpha_{i}}{\kappa_{i}}\left(\operatorname{sgn}(v)-\frac{F_{i}}{\alpha_{i} s(v)}\right)
$$

where $C$ is an attraction constant, that defines how fast $\left|F_{i}\right|$ converges to $\alpha_{i} s(v)$. The element remains in the sliding regime until the velocity is reversed or is equal to zero. In this thesis, 3 Maxwell massless elements are used in the GMS model, which is enough to model mechanical systems with a fair precision, as seen in the work of Piatkowski (2014). It is important to emphasize that the application of this model in diaphragm control valves was never carried out before, being a relevant contribution of this work, since the dynamical models tend to be more precise for predicting systems that present low velocities (PIATKOWSKI, 2014).

In steady state, the Maxwell element displacement tends to $\psi_{i} \rightarrow \alpha_{i} s(v) \operatorname{sgn}(v) / \kappa_{i}$.

\subsubsection{The Dwell Time}

The models described previously do not include a prediction for a $F_{s}$ increase due to higher dwell times, however, this can be modeled by allowing $F_{s}$ to vary with time, as 
suggested by (ARMSTRONG-HÉLOUVRY; DUPONT; CANUDAS de WIT, 1994):

$$
F_{s}\left(t_{d t}, \lambda\right)=F_{s_{0}}+\left(F_{s_{\infty}}-F_{s_{0}}\right) \frac{t_{d t}}{t_{d t}+\lambda}
$$

where $t_{d t}$ is the dwell time, $F_{s_{0}}$ is the static friction force when the system has just reached the sticking state, $F_{s_{\infty}}$ is the static friction force when the system remained sticking for a long period and $\lambda$ is a constant that is inversely proportional to how fast the static friction force increases from $F_{s_{0}}$ to $F_{s_{\infty}}$. This behavior is neglected in this work, since the measurement noise and control loop perturbations usually results in frequent controlled variable corrections and thus stem movements.

\subsection{Diaphragm Valve Model Discretization}

The discretization of the valve and friction models discussed previously is fundamental for simulation and parameters estimation. In this section the models are discretized and the generic friction models presented in previous sections are modified to comply with the valve model. This is another contribution of this thesis.

\subsubsection{Valve Discrete Model}

From Eq. (3.1), the resultant force can be defined as:

$$
F_{\text {res }}(t)=S_{a} P(t)-k x(t)-F_{f}(\dot{x}, t)-F_{\text {init }} .
$$

The valve stem acceleration is:

$$
a(t)=\ddot{x}(t)=\frac{F_{\text {res }}(t)}{m} .
$$

The valve stem velocity $(\dot{x})$ and position $(x)$ can be calculated easily, by integrating the valve stem acceleration. The numerical integration can be done using the bilinear transformation (FADALI; VISIOLI, 2013):

$$
\begin{aligned}
v[n+1]=\dot{x}[n+1] & \approx \frac{T_{s}}{2} \frac{1+q^{-1}}{1-q^{-1}} \ddot{x}[n] \\
x[n] & \approx \frac{T_{s}}{2} \frac{1+q^{-1}}{1-q^{-1}} \dot{x}[n],
\end{aligned}
$$

here $n \in \mathbb{N}, x[n]=x\left(n T_{s}\right)$ is the discrete signal, $q$ is the time shift operator and $T_{s}$ is the sampling time. The valve discrete model may include the physical stem constraints, $x_{\text {min }}$ and $x_{\max }$, to prevent valve movements outside these limits. If these limits are reached, the resultant force, stem acceleration, velocity and position must be clipped accordingly.

The I/P converter is assumed to be a generic first order discrete equation:

$$
P[n+1]=a_{0} P[n]+b_{0}+b_{1} \mathrm{OP}[n]
$$


where $a_{0}, b_{0}$ and $b_{1}$ are constants related to Eq. (3.4) parameters:

$$
\begin{aligned}
\tau_{\mathrm{I} / \mathrm{P}} & =-\frac{T_{s}}{\ln a_{0}} \\
P_{\min } & =\left(b_{0} \cdot \tau_{\mathrm{I} / \mathrm{P}}\right) / T_{s} \\
K_{P_{g}} & =\left(b_{1} \cdot \tau_{\mathrm{I} / \mathrm{P}}\right) / T_{s} .
\end{aligned}
$$

\subsubsection{Karnopp Discrete Model}

The Karnopp model has no dynamics, therefore its use is straightforward. However, inspired by the GMS model, there are some improvements that can be made to achieve more efficient simulations, which is also a small contribution of this work. The discrete Karnopp model is defined as:

$$
F_{f}[n]= \begin{cases}\operatorname{sgn}\left(F_{r}[n]\right) \cdot \min \left(F_{s},\left|F_{r}[n]\right|\right) & , \text { when in presliding state } \\ F_{d}(\dot{x}[n]) & , \text { when in sliding state }\end{cases}
$$

here $F_{r}[n]=S_{a} P[n]-k x[n]-F_{\text {init }}$ and $F_{d}(\dot{x}[n])$ is directly obtained from Eq. (3.8). The sliding regime is active when $\left|F_{f}[n]\right|$ is higher than $F_{s}$ and the stem accelerates. The presliding regime is active when it is detected a movement reversal or the system stops, which can determined when the following equation is true:

$$
\dot{x}[n] \cdot \dot{x}[n-1] \leq 0 \text {. }
$$

To increase the simulation precision, it is necessary to set $\dot{x}[n]=0$, when the presliding state is detected.

Note that the Karnopp model used in this work is different from the original Karnopp model. As the differences does not significantly modifies the model dynamics, the model is still referred as the Karnopp model.

\subsubsection{LuGre Discrete Model}

The discrete derivative of the average bristle deflection is obtained directly from Eq. (3.10):

$$
\dot{z}[n+1]=\dot{x}[n]-\frac{|\dot{x}[n]|}{g(\dot{x}[n])} z[n],
$$

with $g(\dot{x}[n])$ derived directly from Eq. (3.11). Correspondingly, the average bristle deflection is obtained by the numerical integration of its derivative:

$$
z[n] \approx \frac{T_{s}}{2} \frac{1+q^{-1}}{1-q^{-1}} \dot{z}[n] .
$$

The friction force is calculated as presented in Eq. (3.9). 


\subsubsection{GMS Discrete Model}

The GMS discrete model development is a combination of the Karnopp and LuGre discrete model implementations. The Maxwell massless element is governed by two differential equations, whether in the sliding or presliding states. In the discrete form, these equations are written as:

$$
\begin{cases}\dot{\psi}_{i}[n]=\dot{x}[n] & , \text { when in presliding state } \\ \dot{\psi}_{i}[n+1]=\frac{C \alpha_{i}}{\kappa_{i}}\left(\operatorname{sgn}(\dot{x}[n])-\frac{F_{i}[n]}{\alpha_{i} s(\dot{x}[n])}\right) & , \text { when in sliding state }\end{cases}
$$

The position of the Maxwell elements can be determined by the numerical integration of $\psi_{i}$, similarly as shown in Eq. (3.25). The friction force is calculated directly from Eq. (3.12).

The Maxwell elements are considered in the presliding state when there is a movement reversal, which is detected as presented in Eq. (3.23). The sliding state is active when $F_{i}[n] \geq \alpha_{i} s(\dot{x}[n])$.

\subsubsection{Sampling Time Selection}

When simulating discrete systems, the sampling time design is important, since it can lead to simulation failures. The LuGre and GMS models consider that friction force when in presliding state is a consequence of relative movements, with typical magnitudes of tens of micrometers (ARMSTRONG-HÉLOUVRY; DUPONT; CANUDAS de WIT, 1994). This phenomenon usually imposes the most restrictive requirements for specifying the sampling time. By using the rectangular integration, it is possible to guarantee that the maximum stem position change in a time step is smaller than a constant:

$$
T_{s}\left|v_{\text {max }}\right|<x_{\text {mchange }},
$$

where $v_{\text {max }}$ is the maximum stem velocity and $x_{\text {mchange }}$ is the desired maximum position change in a time step. Usually, the valve bandwidth is limited by the I/P converter dynamics, therefore a good estimate for the maximum stem velocity, considering sinusoidal excitation signals, is:

$$
v_{\max } \approx\left(x_{\max }-x_{\min }\right) \frac{2 \pi}{\tau_{\mathrm{I} / \mathrm{P}}}
$$

with $x_{\max }$ and $x_{\min }$ the maximum and minimum stem position, respectively. To determine the value of $x_{\text {mchange }}$, it suffices to note that for the vast majority of applications, $5 \mathrm{\mu m}$ is at least a third of the maximum displacement during presliding.

As the Karnopp model has no dynamics during presliding regime, Eq. (3.27) can be rewritten by considering that a conservative criterion is to set the maximum stem movement in a time step equal to a thousandth of the valve stroke:

$$
T_{s} v_{\max }<\frac{x_{\max }-x_{\min }}{1000}
$$


With the same estimation for the maximum stem velocity as in Eq. (3.28), the sampling time criteria for the Karnopp model reduces to:

$$
T_{s}<\frac{\tau_{\mathrm{I} / \mathrm{P}}}{2000 \pi}
$$

\subsection{Data Driven Model of Pneumatic Control Valves}

Amidst the empirical models, the Kano model can be considered the most suitable to predict the behavior of pneumatic control valves (GARCIA, 2008). As other data driven models, its design was inspired by the valve signature, which is obtained by varying the $\mathrm{OP}$ in a trapezoidal manner and plotting the stem position or valve flow rate in the $y$-axis and the diaphragm pressure or OP in the x-axis. Figure 3.10 presents a typical idealized valve signature. The Kano model considers that the valve behavior can be defined by a deadband $(S)$ and a slip band $(J)$. When the direction of the movement is reversed, the deadband has to be surpassed, as shown in position $d$. If the valve stops and the movement is to be initiated in the same direction as before, only the slip band has to be overcome, as shown in position $c$. This model neglects the effect of the viscous friction and considers that the transition from presliding to sliding occurs instantaneously, with a jump from the static friction force to Coulomb friction force, as shown in the path from $a$ to $b$. Note that the Kano model simplifies the valve dynamics model, by considering instantaneous stem movements when OP varies and simplifies the friction model, neglecting many friction characteristics. The main advantage of the Kano model is that it operates at the same sampling time of the controller output, resulting in effective simulations.

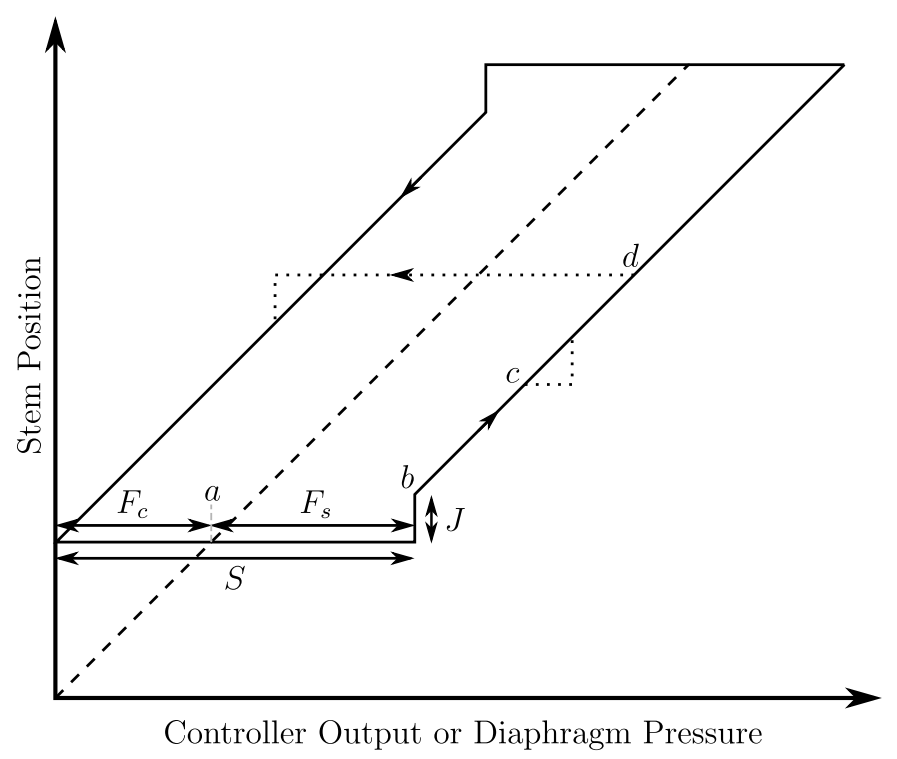

Figure 3.10 - A typical valve signature curve.

Source: Based on Kano et al. (2004), Choudhury, Thornhill and Shah (2005).

The Kano model implementation is presented in Algorithm 3.1. In this thesis, the Kano model differs from the original algorithm, since it uses another parameter, $D$, which 
simulates the effect of the initial force and uses a proper output function to match the valve stem position. This is another small contribution of this work. Yet, the model input is considered the normalized diaphragm pressure, since it is not physically feasible to have instantaneous stem movements with changes in OP. Note that for increase the simulation performance, the input has to be normalized. Observe that the stem limits shown in Kano algorithm are not the physical limits, but the maximum $\left(x_{\max P}\right)$ and minimum $\left(x_{\min \mathrm{P}}\right)$ stem positions when the diaphragm pressure is maximum and minimum, respectively.

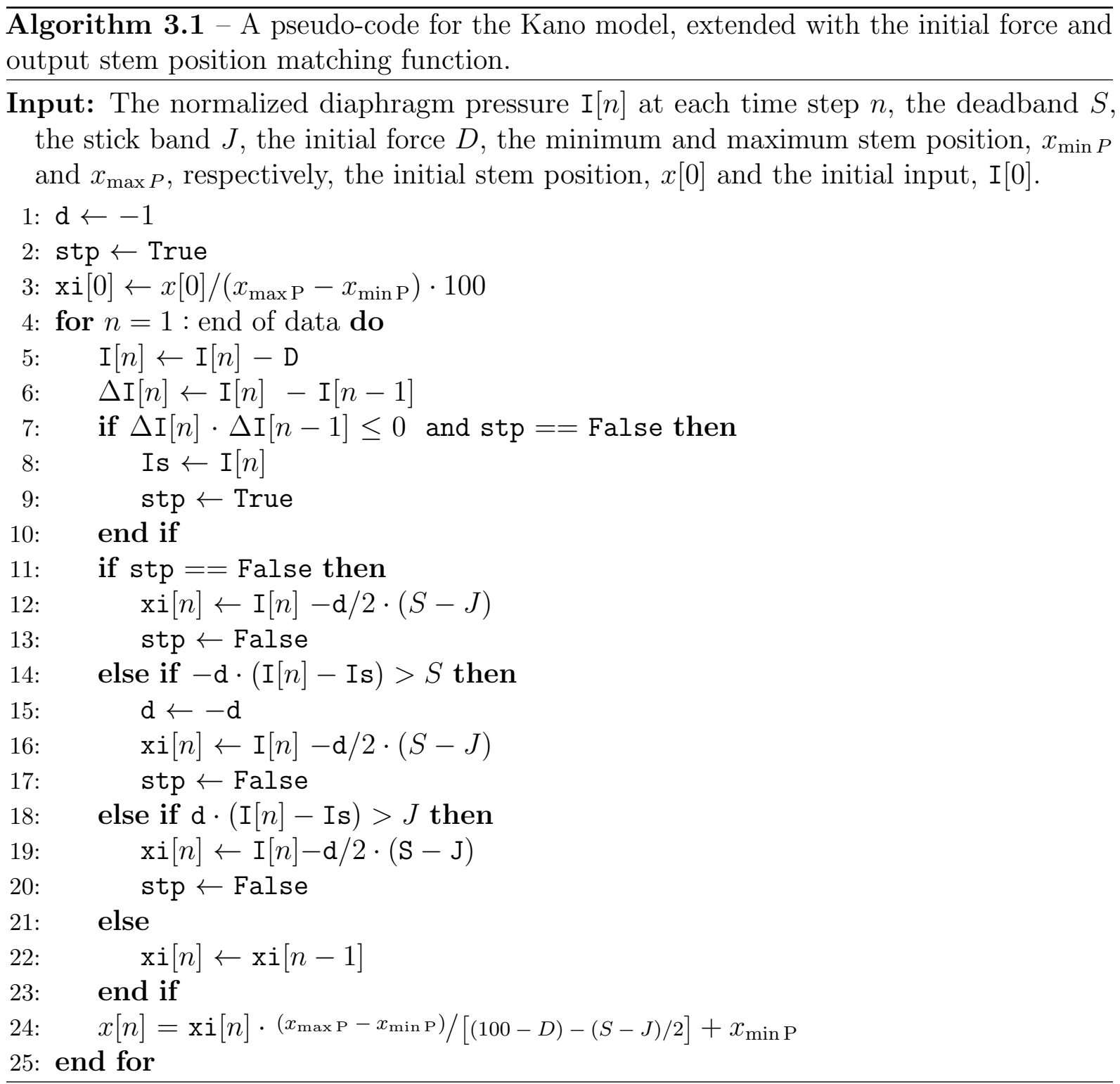


The Kano parameters are related to Stribeck curve and valve parameters:

$$
\begin{aligned}
S & =\frac{2 F_{s}}{\left(P_{\max }-P_{\min }\right) \cdot S_{a}} \cdot 100 \\
J & =\frac{F_{s}-F_{c}}{\left(P_{\max }-P_{\min }\right) \cdot S_{a}} \cdot 100 \\
D & =\frac{F_{\text {init }} / S_{a}-P_{\min }}{P_{\max }-P_{\min }} \cdot 100 .
\end{aligned}
$$

The main difference from the relationships shown in Eq. (3.31) and the ones proposed by Uehara, Garcia and Romano (2008) is that the latter considered that when the stem is moving, the friction force is $F_{c}$. However, this is not true if one analyzes the Stribeck curve, as when the valve velocity is close to zero, the friction force is close to $F_{s}$, leading to the equivalence equation presented in Eq. (3.31).

\subsection{Diaphragm Valve Simulation}

To ensure that the discrete models are equivalent to their continuous counterparts, simulations were performed and the results of the continuous and discrete models were compared, using a sampling time $T_{s}=10 \mu \mathrm{s}$, except for the Kano model, which employed $T_{s}=1 \mathrm{~ms}$. The valve and the friction parameters used in the simulations are presented in Tables 3.1 and 3.2 .

Table 3.1 - Valve parameters used in the simulations.

\begin{tabular}{cc}
\hline Parameter & Value \\
\hline$\tau_{\mathrm{I} / \mathrm{P}}(\mathrm{s})$ & $1 / 0.28$ \\
$S_{a}\left(\mathrm{~m}^{2}\right)$ & $445 \cdot 10^{-4}$ \\
$P_{\max }(\mathrm{Pa})$ & 180000 \\
$P_{\min }(\mathrm{Pa})$ & 41368 \\
$m(\mathrm{~kg})$ & 1.6 \\
$k(\mathrm{~N} / \mathrm{m})$ & 210490 \\
$F_{\text {init }}(\mathrm{N})$ & 2550 \\
$x_{\min }(\mathrm{m})$ & 0 \\
$x_{\max }(\mathrm{m})$ & 0.029 \\
$x_{\min \mathrm{P}}(\mathrm{m})$ & 0 \\
$x_{\max }(\mathrm{m})$ & 0.029 \\
\hline
\end{tabular}

Source: from Author. 
Table 3.2 - Friction parameters used in the simulations.

\begin{tabular}{cc|cc}
\hline Parameter & Value & Parameter & Value \\
\hline$F_{c}(\mathrm{~N})$ & 700 & $\alpha_{1}$ & 0.75 \\
$F_{s}(\mathrm{~N})$ & 780 & $\alpha_{2}$ & 0.2 \\
$F_{v}(\mathrm{Ns} / \mathrm{m})$ & $1.25 \cdot 10^{5}$ & $\alpha_{3}$ & 0.05 \\
$v_{s}(\mathrm{~m} / \mathrm{s})$ & $5 \cdot 10^{-4}$ & $\kappa_{1}(\mathrm{~N} / \mathrm{m})$ & $F_{s} \alpha_{1} / 2 \cdot 10^{-5}$ \\
$D V(\mathrm{~m} / \mathrm{s})$ & $v_{s} / 100$ & $\kappa_{2}(\mathrm{~N} / \mathrm{m})$ & $F_{s} \alpha_{2} / 10^{-5}$ \\
$\sigma_{0}(\mathrm{~N} / \mathrm{m})$ & $F_{s} / 3 \cdot 10^{-5}$ & $\kappa_{3}(\mathrm{~N} / \mathrm{m})$ & $F_{s} \alpha_{3} / 5 \cdot 10^{-6}$ \\
$\sigma_{1}(\mathrm{Ns} / \mathrm{m})$ & $4 \sqrt{\sigma_{0}}$ & $\nu_{1}(\mathrm{Ns} / \mathrm{m})$ & $\sqrt{k+\kappa_{1}+\kappa_{2}+\kappa_{3}} / 3$ \\
$S(\%)$ & 25.29 & $\nu_{2}(\mathrm{Ns} / \mathrm{m})$ & $\sqrt{k+\kappa_{1}+\kappa_{2}+\kappa_{3}} / 6$ \\
$J(\%)$ & 1.2968 & $\nu_{3}(\mathrm{Ns} / \mathrm{m})$ & $\sqrt{k+\kappa_{1}+\kappa_{2}+\kappa_{3}} / 2$ \\
$D(\%)$ & 11.50 & $C(\mathrm{rad} / \mathrm{s})$ & 20.00 \\
\hline
\end{tabular}

Source: from Author.

The simulation excitation signal, OP, consisted in a sequence of ramps with random velocities and magnitudes, as shown in Figure 3.11.

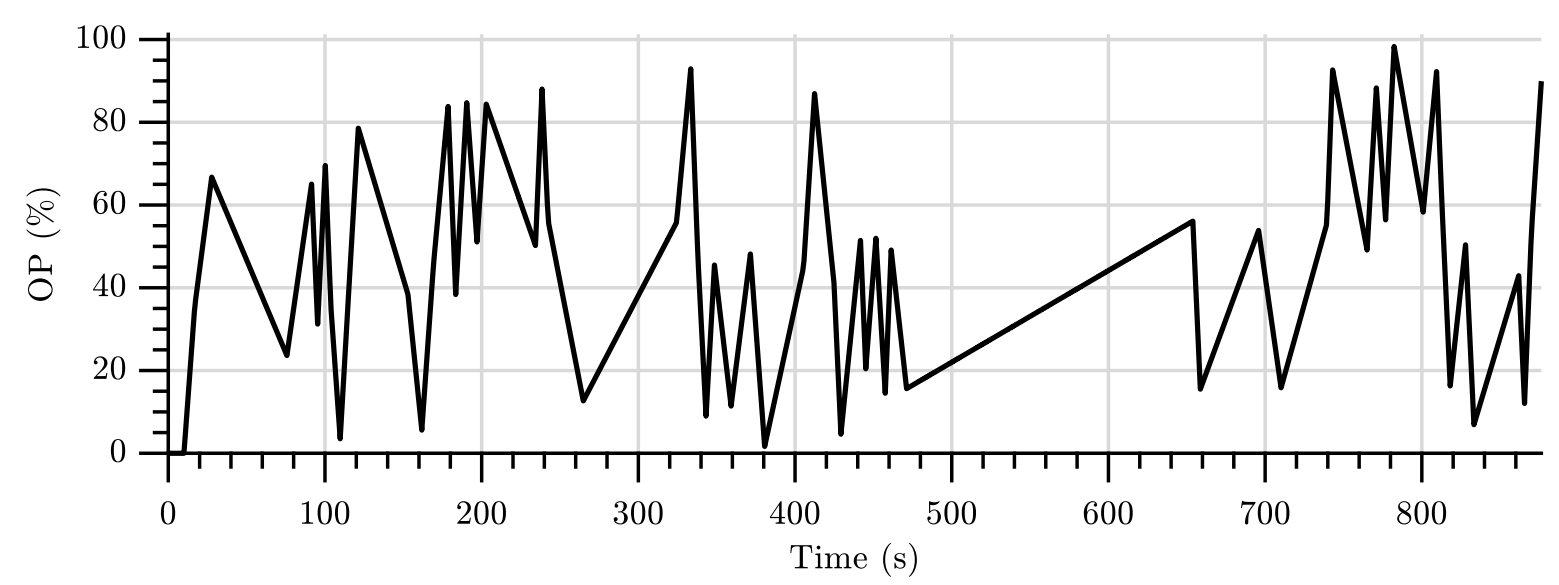

Figure 3.11 - Excitation signal used in the simulations.

Source: from Author.

The Karnopp, LuGre and GMS discrete and continuous simulation results are presented in Figure 3.12, where no visual difference is distinguished from any of the six simulations. The Kano simulation result is compared to the Karnopp model as shown in Figure 3.13, where it is possible to verify that the models present similar results, except for a variable offset. 


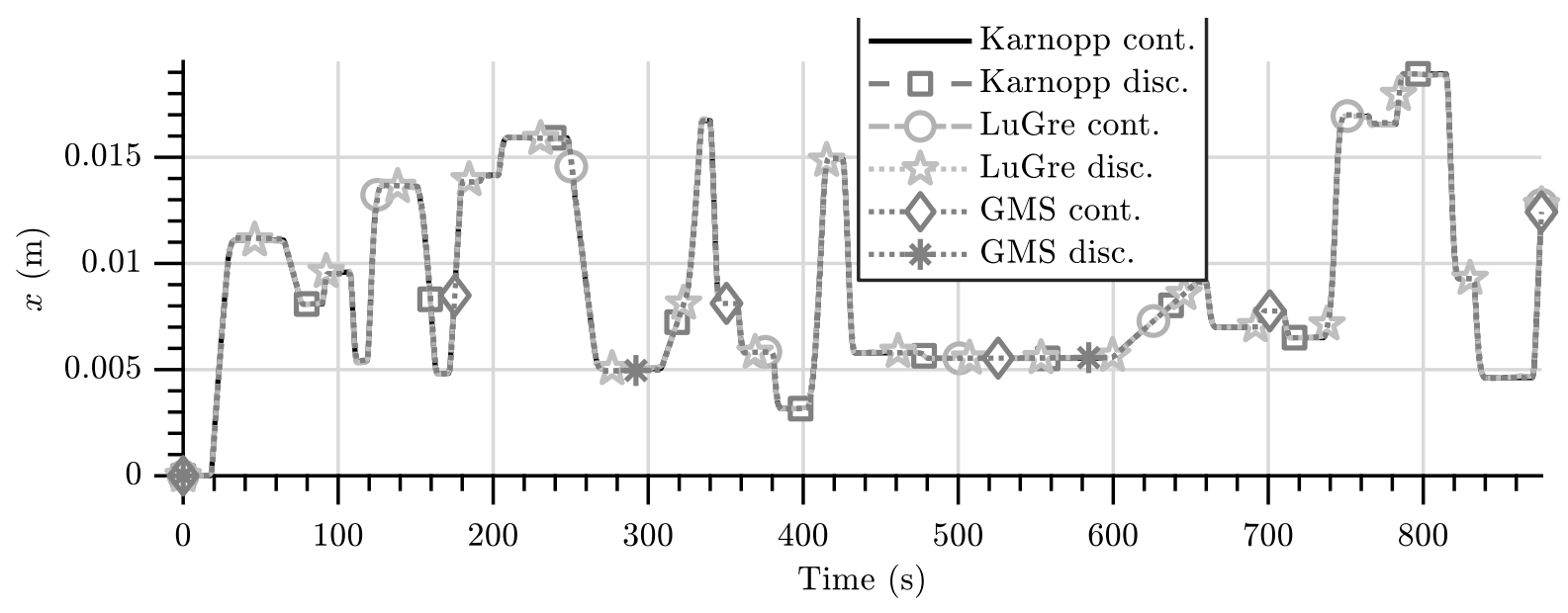

Figure 3.12 - Comparison of continuous and discrete valve models with Kano, Karnopp, LuGre and GMS friction models.

Source: from Author.

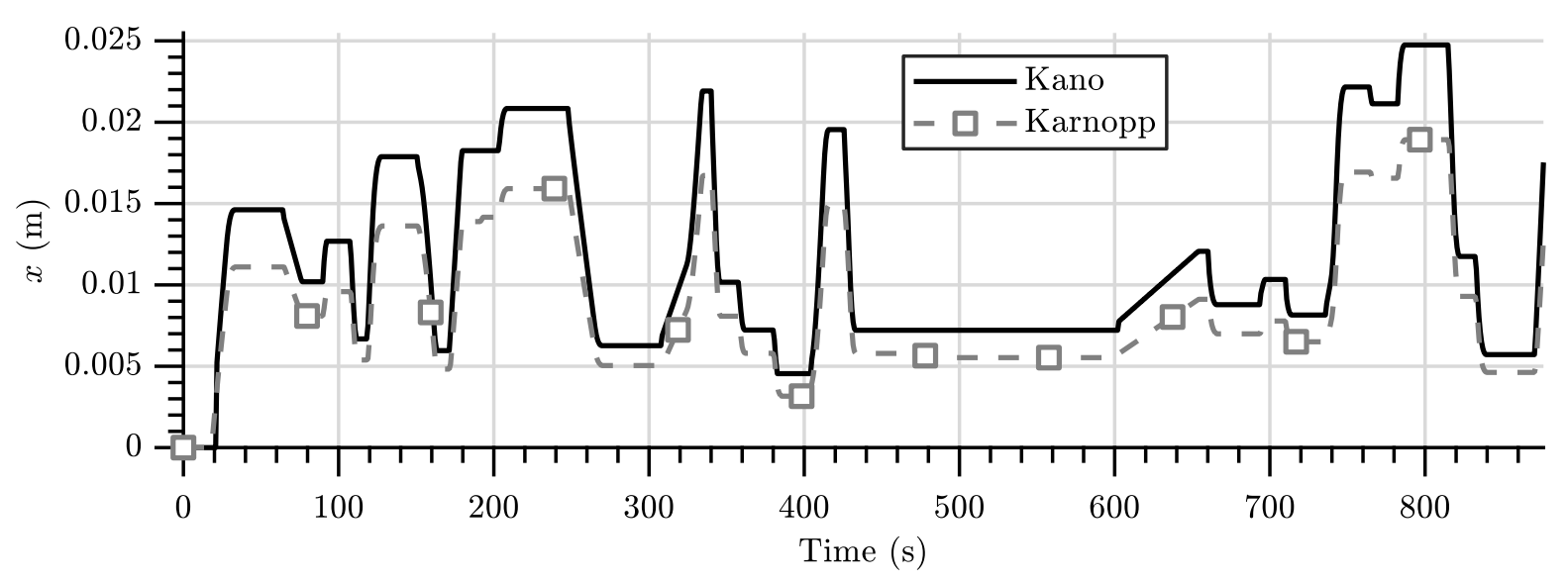

Figure 3.13 - Simulation of the valve using the Kano model and comparison with the Karnopp model.

Source: from Author.

For a better evaluation of the discretization, the quantitative $f i t$ index is calculated, defined as:

$$
f i t=100 \cdot\left(1-\frac{\sqrt{\sum_{n=1}^{n_{f}}\left(x_{c}[n]-x[n]\right)^{2}}}{\sqrt{\sum_{n=1}^{n_{f}}\left(x_{c}[n]-\mu\left(x_{c}\right)\right)^{2}}}\right) \text {, }
$$

where $x_{c}[n]$ is the continuous model stem position at timestep $n, \mu\left(x_{c}\right)$ is the mean value of $x_{c}$ and $n_{f}$ is the final time step. As per Eq. (3.29), the Karnopp sampling time can be lower, the $f$ it was calculated with $T_{s}=10^{-4} \mathrm{~s}$, as well. The quantitative comparison is presented in Table 3.3, where it is possible to conclude that the discretization is successful. The simulation time shows a huge difference between the data driven and first principle models, being the former much more computationally efficient. 
Table 3.3 - Continuous and discrete valve models comparison.

\begin{tabular}{ccc} 
Model & fit & Simulation Time $(\mathrm{s})$ \\
\hline Kano $\left(T_{s}=10^{-3} \mathrm{~s}\right)$ & - & 0.1 \\
Karnopp $\left(T_{s}=10^{-4} \mathrm{~s}\right)$ & $97.68 \%$ & 1.9 \\
Karnopp $\left(T_{s}=10^{-5} \mathrm{~s}\right)$ & $100 \%$ & 15.4 \\
LuGre $\left(T_{s}=10^{-5} \mathrm{~s}\right)$ & $100 \%$ & 20.3 \\
GMS $\left(T_{s}=10^{-5} \mathrm{~s}\right)$ & $100 \%$ & 25.0 \\
\hline \multicolumn{3}{c}{ Source: from Author. }
\end{tabular}




\section{Open Loop Parameters Estimation}

This chapter addresses the design of an open loop diaphragm valve parameters estimation method. In this case, the valve stem position is allowed to move at will, as it is not being used in a control loop or is in a maintenance bench.

By evaluating the valve model of Eq. (3.1), one can easily identify the parameters that have to be estimated: the diaphragm cross sectional area, $S_{a}$, the moving parts mass, $m$, the spring constant, $k$, the initial force, $F_{\text {init }}$, the I/P converter model parameters and the friction parameters, $F_{f}$, depending on the selected friction model (Section 3.2). Regarding the Kano data driven model (Section 3.3), the estimated parameters are related to the I/P converter model, valve deadband, $S$, slip band, $J$, initial force, $D$ and maximum and minimum observed stem positions, $x_{\min \mathrm{P}}$ and $x_{\max \mathrm{P}}$, respectively.

Estimating the valve and friction parameters can be accomplished by minimizing the difference between the measured and simulated stem position, e.g. calculating the mean squared error of a given dataset:

$$
\left\{\hat{S}_{a}, \hat{m}, \hat{k}, \hat{F}_{\text {init }}, \hat{\theta}_{\mathrm{I} / \mathrm{P}}, \hat{\theta}_{f}\right\}=\underset{S_{a}, m, k, F_{\text {init }}, \theta_{\mathrm{I} / \mathrm{P}}, \theta_{f}}{\arg \min } V_{n_{f}}\left(S_{a}, m, k, F_{\text {init }}, \theta_{\mathrm{I} / \mathrm{P}}, \theta_{f}\right)
$$

here the caret denotes estimated parameter, $\theta_{\mathrm{I} / \mathrm{P}} \in \mathbb{R}^{3}$ is a vector with the discrete $\mathrm{I} / \mathrm{P}$ model parameters, $\theta_{f} \in \mathbb{R}^{d_{f}}$ is the friction model parameters vector, whose dimension $\left(d_{f}\right)$ depends on which model is used in the simulation, $n_{f}$ is the number of samples and $V_{n_{f}}$ is defined as the mean squared prediction error (some function arguments are omitted for simplicity):

$$
V_{n_{f}}\left(S_{a}, m, k, F_{\text {init }}, \theta_{\mathrm{I} / \mathrm{P}}, \theta_{f}\right)=\frac{1}{n_{f}} \sum_{n=1}^{n_{f}} e_{x}\left(n, \mathrm{OP}[n], \theta_{\mathrm{I} / \mathrm{P}}, P[0], x[0], \dot{x}[0], S_{a}, m, k, F_{\text {init }}, \theta_{f}\right)^{2},
$$

where $\mathrm{OP}[n]$ is the controller output, $P[0]$ is the initial diaphragm pressure, $x[0]$ is a vector with initial stem position and initial internal friction states position, when applicable, $\dot{x}[0]$ is a vector with initial stem velocity and initial internal friction states velocity, when applicable. The prediction error is calculated as:

$$
e_{x}(n)=\hat{x}\left(n, \mathrm{OP}[n], \theta_{\mathrm{I} / \mathrm{P}}, P[0], x[0], \dot{x}[0], S_{a}, m, k, F_{\text {init }}, \theta_{f}\right)-x[n],
$$

here $\hat{x}\left(n, \mathrm{OP}[n], \theta_{\mathrm{I} / \mathrm{P}}, P[0], x[0], \dot{x}[0], S_{a}, m, k, F_{\text {init }}, \theta_{f}\right)$ is the simulated stem position, using a given valve and friction model and $x[n]$ is the measured stem position.

As mentioned in Chapter 2, the diaphragm pressure is a measured variable, therefore the I/P model parameters can be estimated separately:

$$
\hat{\theta}_{\mathrm{I} / \mathrm{P}}=\underset{\theta_{\mathrm{I} / \mathrm{P}}}{\arg \min } \frac{1}{n_{f}} \sum_{n=1}^{n_{f}}\left(\hat{P}\left(n, \mathrm{OP}[n], P[0], \theta_{\mathrm{I} / \mathrm{P}}\right)-P[n]\right)^{2},
$$


where $\hat{P}\left(n, \mathrm{OP}[n], P[0], \theta_{\mathrm{I} / \mathrm{P}}\right)$ is the simulated diaphragm pressure and $P[n]$ is the measured diaphragm pressure.

As the valve moving parts mass and diaphragm section area can be informed by the vendor and tends to be constant during the valve life time, these parameters are considered to be known. Therefore, Eq. (4.1) can be simplified to:

$$
\left\{\hat{k}, \hat{F}_{\text {init }}, \hat{\theta}_{f}\right\}=\underset{k, F_{\text {init }}, \theta_{f}}{\arg \min } V_{n_{f}}\left(S_{a}, m, k, F_{\text {init }}, \theta_{f}\right),
$$

with the prediction error reduced to:

$$
e_{x}(n)=\hat{x}\left(n, P[n], x[0], \dot{x}[0], S_{a}, m, k, F_{\text {init }}, \theta_{f}\right)-x[n]
$$

It is worth noting that in the proposed estimation framework, $\hat{x}$ is independent from past stem measurements, leading to free simulation prediction minimization.

Despite solving Eq. (4.5) lead to the parameters estimation, there are many open questions that need to be answered beforehand. The optimization dimension is high, resulting in, at most, 16 estimated parameters, thus is it possible to estimate some of said parameters a priori to lower the complexity? Which excitation signal to use for estimating the parameters? Is it possible to define search space for the parameters? Lastly, which optimization method to use, since it is potentially a nonlinear, discontinuous and non convex set? Those questions are to be addressed in subsequent sections.

\subsection{A Priori Estimation of Parameters}

An initial estimate of the valve deadband, $S_{0}$, can be obtained by increasing OP to $90 \%$ and then decreasing it linearly, with a low rate of change until it is observed a negative stem position velocity. The suggested OP rate of change is $1 \% / \mathrm{s}$, since slower variations may lead to time consuming experiments and faster may lead to delays between $O P$ and the diaphragm pressure. The parameter $S$ is the difference between $90 \%$ and the controller output at the moment which the valve movement is detected, as presented in Algorithm 4.1. Preferably, the normalized diaphragm pressure should be used in this estimation. This parameter is easy to identify and can be useful for designing the excitation signals and deriving a first initial guess $\left(S_{0}\right)$ for the other model friction parameters. A simulated example is shown in Figure 4.1. 


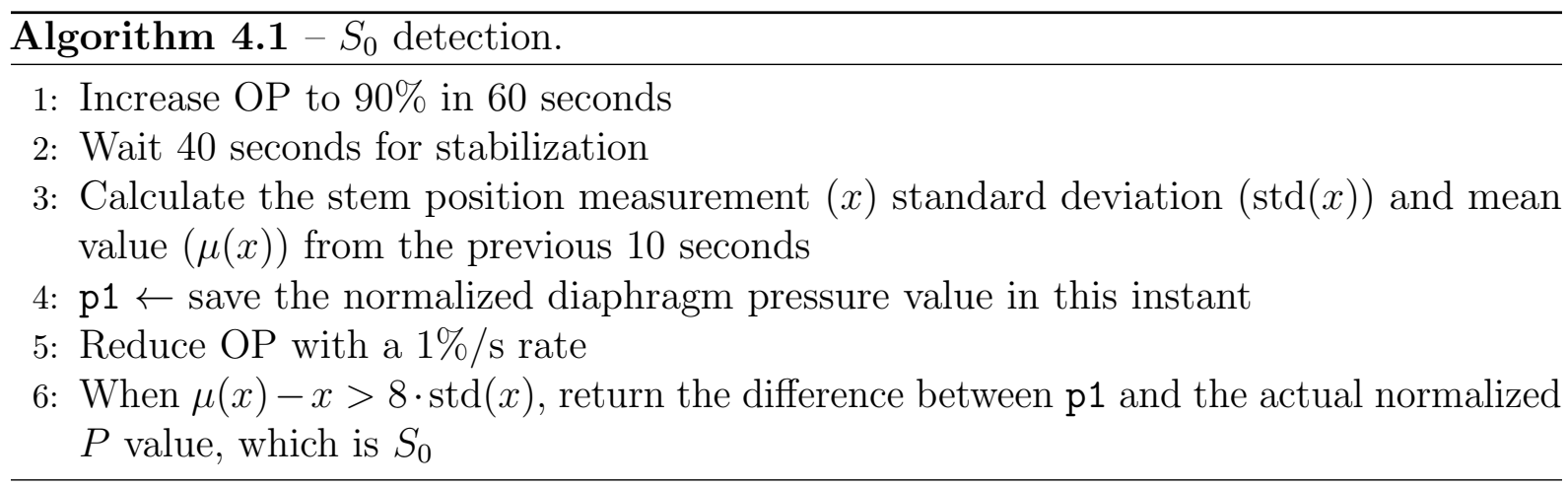

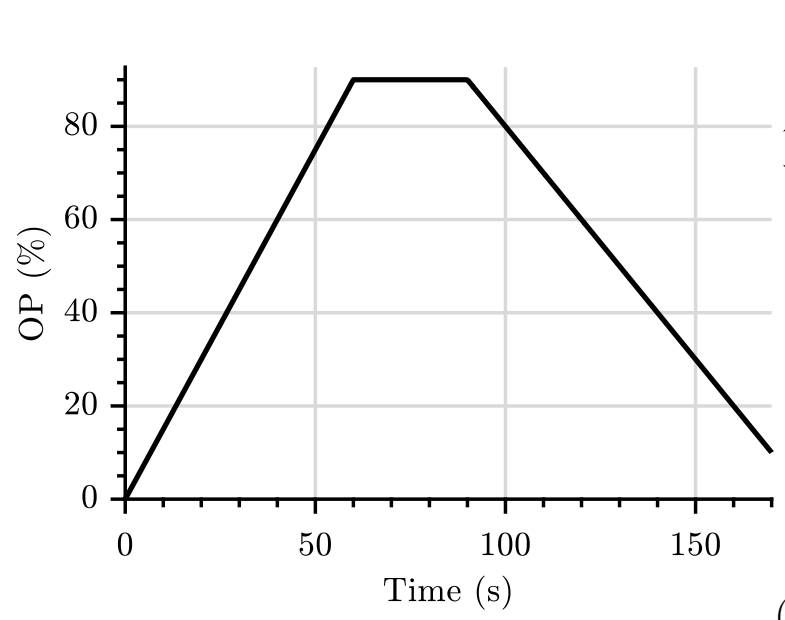

(a) Controller output (OP).

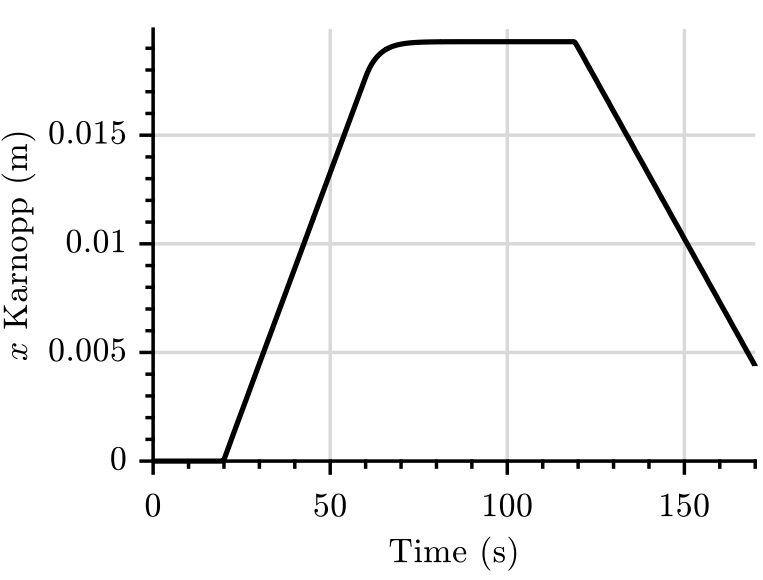

(b) Valve stem position given by Karnopp friction model.

Figure 4.1 - Simulated example for estimating $S_{0}$ a priori.

Source: from Author.

The I/P converter parameters can be estimated by minimizing the difference between the measured and the simulated diaphragm pressure as presented in Eq. (4.4), such that the latter is calculated using the discrete model given by Eq. (3.20). The solution for Eq. (4.4) is easily given using the one step ahead prediction minimization Least Squares Estimator (LSE) (LJUNG, 1999). However, it is preferable to use the infinite steps ahead prediction error minimization with an unconstrained optimization method to estimate the parameters vector. In this work, it was used the Simplex optimization method (NELDER; MEAD, 1965), with $\tau_{\mathrm{I} / \mathrm{P}}=1 \mathrm{~s}$ and $P_{\min }$ and $K_{P_{g}}$ the same as the valve vendor manual as an initial guess. As the valve mechanical system dynamics tends to be faster than the I/P converter, the valve bandwidth can be approximated by the I/P bandwidth $\left(1 / \tau_{\mathrm{I} / \mathrm{P}} \mathrm{rad} / \mathrm{s}\right)$, that can be calculated from the estimated discrete filter parameters using Eq. (3.21). Even though the first order filter is simple to simulate and easy to estimate, it must be noted that it is not perfect for a precise I/P converter simulation (MARQUES; GARCIA, 2019). For example, the I/P dynamics tends to be faster when excited with ramp signals than using steps and presents overshoot for positive steps but not for negative steps, as shown in Figure 4.2. 


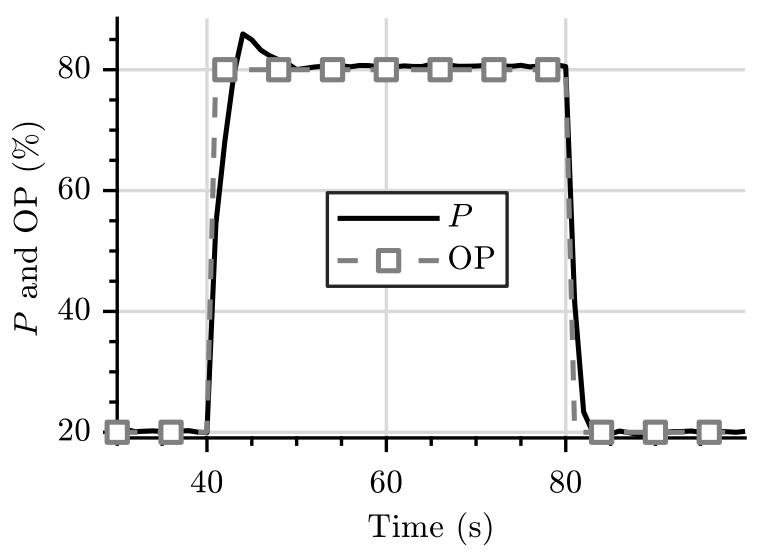

(a) Rectangular signal excitation.

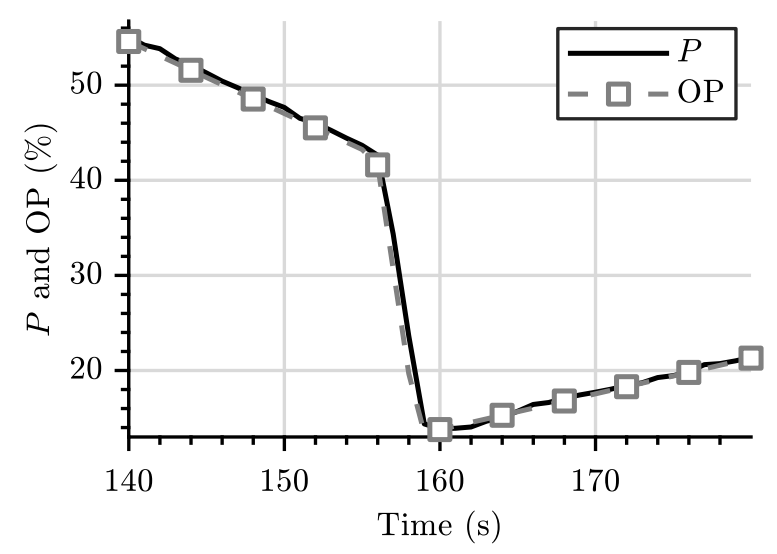

(b) Ramp signals excitation.

Figure $4.2-\mathrm{I} / \mathrm{P}$ converter dynamics experimental data using the graphite gasket valve.

Source: from Author.

It is suggested to use both step and ramp signals for estimating the valve I/P model parameters. The step signal is rectangular with $80 \mathrm{~s}$ period, $60 \%$ magnitude, starting with $20 \%$, as in Figure $4.2 \mathrm{a}$. The $\mathrm{I} / \mathrm{P}$ time constant obtained with the step signal, $\tau_{\mathrm{I} / \mathrm{P}_{s}}$, is the one used for excitation signals and filters design. The time constant obtained using the ramp signals, $\tau_{\mathrm{I} / \mathrm{P}_{r}}$, can be estimated using the excitation signal used for estimate $k$ and $F_{\text {init }}$ that is presented in the following paragraphs. Even though the two estimated models are not perfect and reflect the linear approximation of the $\mathrm{I} / \mathrm{P}$ converter in the experiment, the model imprecision is not of great importance, as it is used mainly for secondary objectives and to detect faults in the I/P converter system.

The valve $k$ and $F_{\text {init }}$ can be estimated a priori also, reducing the number of estimated parameters of Eq. (4.5). The valve $k$ and $F_{\text {init }}$ parameters estimation technique suggested in this thesis is inspired in previous works (GARCIA, 2007; ROMANO; GARCIA, 2007) and is based on the diaphragm valve model analysis when the stem presents a constant velocity. If the valve is excited with a ramp signal, the result is an approximately constant stem velocity, therefore, Eq. (3.1) reduces to:

$$
0=S_{a} P(t)-k x(t)-F_{f}(\dot{x})-F_{\text {init }}
$$

In steady state, $F_{f}(\dot{x})$ is constant, therefore Eq. (4.7) reduces to:

$$
S_{a} P(t)=k x(t)+F_{b_{l}}\left(\dot{x}_{b_{l}}\right),
$$

here $F_{b_{l}}\left(\dot{x}_{b_{l}}\right)=F_{f}\left(\dot{x}_{b_{l}}\right)+F_{\text {init }}$ is a constant force, $\dot{x}_{b_{l}}$ is a constant velocity, corresponding to a dataset $l \in \mathbb{N}$, which only contains data with a certain constant stem velocity. Therefore, estimating $k$ and $F_{b_{l}}\left(\dot{x}_{b_{l}}\right)$, is reduced to an algebraic linear regression estimation problem:

$$
\mathbf{F}_{\mathbf{d} l}=\Phi_{l} \theta_{l}
$$


where $\mathbf{F}_{\mathbf{d}_{\mathbf{l}}} \in \mathbb{R}^{N_{b_{l}}}$ is the diaphragm force column vector of dataset $l$, containing $N_{b_{l}}$ experimental observations, calculated using measured diaphragm pressure, $\theta_{l}=\left[\begin{array}{ll}k_{l} & F_{b_{l}}\end{array}\right]^{\top} \in \mathbb{R}^{2}$ a parameter column vector, $\Phi_{l}=\left[\begin{array}{ll}\mathbf{x}_{l} & \mathbf{v}_{\mathbf{u}}\end{array}\right] \in \mathbb{R}^{N_{b_{l}} \times 2}$ the regression matrix, $\mathbf{x}_{l} \in \mathbb{R}^{N_{b_{l}}}$ the stem position experimental observations and $\mathbf{v}_{\mathbf{u}} \in \mathbb{R}^{N_{b_{l}}}$ a vector of unitary values. Using the LSE, Eq. (4.9) solution is given by:

$$
\hat{\theta}_{l}=\left(\Phi_{l}^{\top} \Phi_{l}\right)^{-1} \Phi_{l}^{\top} \mathbf{F}_{\mathbf{d} l}
$$

By exciting the valve with $l_{n}$ constant velocities (at least 2 positive and 2 negative), it is possible to draw a Stribeck curve using the previous step calculated velocity and friction force. Therefore, it is possible to obtain an initial estimation of the Stribeck curve parameters and the initial force, by minimizing the following error equation:

$$
\left\{\hat{F}_{s}, \hat{F}_{c}, \hat{F}_{v}, \hat{v}_{s}, \hat{F}_{\text {init }}\right\}=\underset{F_{s}, F_{c}, F_{v}, v_{s}, F_{\text {init }}}{\arg \min } \sum_{l=1}^{l_{n}}\left[\hat{F}_{b_{l}}\left(\dot{x}_{b_{l}}\right)-F_{b}\left(F_{s}, F_{c}, F_{v}, F_{\text {init }}, v_{s}, \dot{x}_{b_{l}}\right)\right]^{2}
$$

where $\hat{F}_{b_{l}}\left(\dot{x}_{b_{l}}\right)$ is estimated with Eq. $(4.10), \dot{x}_{b_{l}}$ is the stem velocity of dataset $l$ and the $F_{b}$ function is defined as:

$$
F_{b}\left(F_{s}, F_{c}, F_{v}, F_{\text {init }}, v_{s}, \hat{\dot{x}}_{b_{i}}\right)=F_{d}\left(F_{s}, F_{c}, F_{v}, v_{s}, \hat{\dot{x}}_{b_{i}}\right)+F_{\text {init }} .
$$

The Eq. (4.11) solution can be obtained using a derivative free optimization method, such as the Simplex. Note that this method provides an initial Stribeck curve parameters estimation, which should not be reliable, due to the low number of data points. The valve stem velocity can be approximated applying the backward differentiation. To minimize the measurement noise, the diaphragm pressure and stem position are filtered with a zero phase, second order linear filter, with 0.9 damping ratio and natural frequency $1 / \tau_{\mathrm{I} / \mathrm{P}_{s}}$ (KORMYLO; JAIN, 1974).

For estimating $k$ and $F_{\text {init }}$, the ideal excitation signal is a series of ramps, covering a wide range of velocities, ideally it should involve much lower and much higher velocities than the Stribeck, i.e. lower than $10^{-5} \mathrm{~m} / \mathrm{s}$ and higher than $0.1 \mathrm{~m} / \mathrm{s}$ (ARMSTRONGHÉLOUVRY; DUPONT; CANUDAS de WIT, 1994). However, the valve may impose constraints, specially at high velocities. The most evident constraint is the trade-off between the maximum valve stroke and the maximum stem velocity, as it is necessary to maintain the same excitation signal for more than $5 \tau_{\mathrm{I} / \mathrm{P}} \mathrm{s}$ to achieve a constant stem velocity. An example of excitation signal is presented in Figure 4.3. Note that in this example, the valve deadband, which is roughly $26 \%$, is compensated in the excitation signal, starting from this value before any ramp is applied, so that the experiment does not take too long with low velocity ramps. The signal with constant velocity is maintained during $20 \cdot \tau_{\mathrm{I} / \mathrm{P}}$ seconds or until the maximum stroke is achieved. The minimum velocity corresponds to a velocity of $1 \mu \mathrm{m} / \mathrm{s}$, which in $\mathrm{OP}$ variation is:

$$
v_{\mathrm{OP} \min _{R}}=\frac{10^{-6} \cdot 100}{x_{\max }-x_{\min }}
$$


and the maximum velocity is calculated to ensure that the signal is maintained constant until it reaches the maximum or minimum stem position in at least $5 \tau_{\mathrm{I} / \mathrm{P}}$ :

$$
v_{\mathrm{OP} \max _{R}}=\frac{\left(100-2 \cdot S_{0}\right)}{5 \tau_{\mathrm{I} / \mathrm{P}}} .
$$

The signal is generated to cover logarithmically the range between $v_{\mathrm{OP} \min _{R}}$ and $v_{\mathrm{OP}} \max _{R}$. Note that the ramps are applied to the upper and lower extrema of the valve stem stroke. It is useful to save the time interval in which the valve is excited with ramps, for latter data processing.

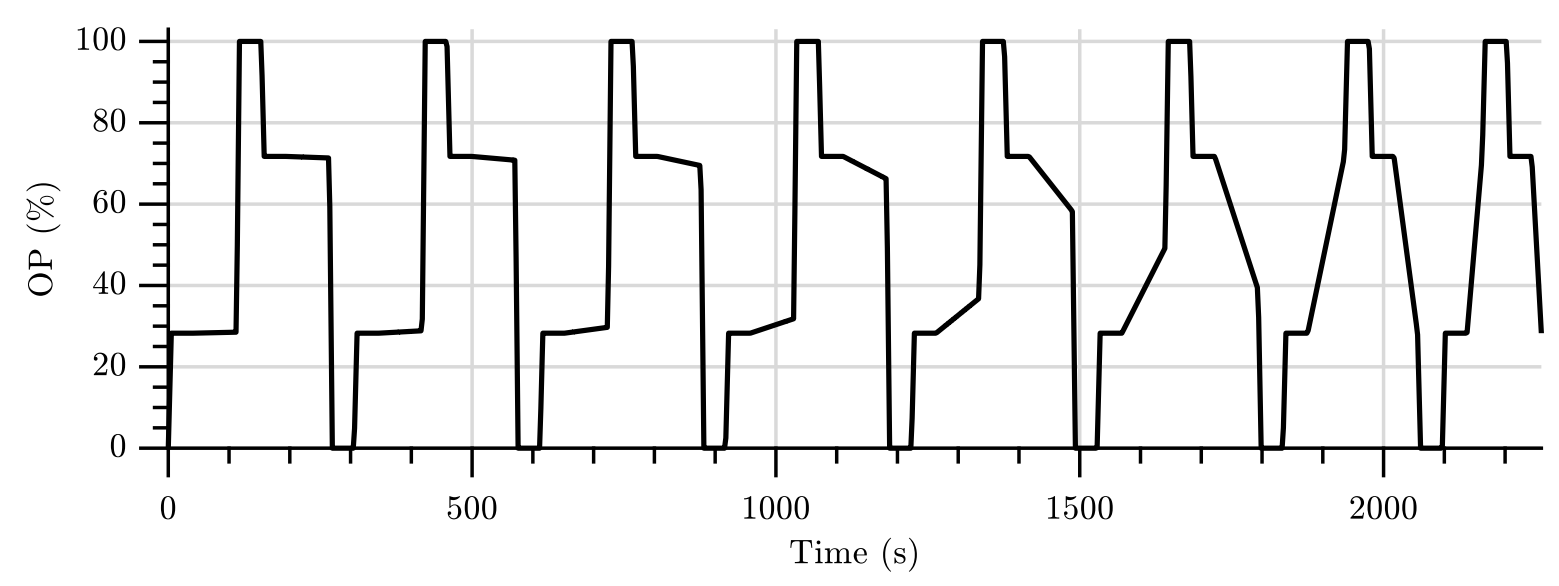

Figure 4.3 - Example of excitation signal used to estimate $k$ and $F_{\text {init }}$ a priori. Source: from Author.

Algorithm 4.2 presents a scheme for estimating $k$ and $F_{\text {init }}$. This method leads to a distribution of $\hat{k}$ estimations for many different valve velocities and stem positions, and it is possible to calculate the mean value of $\hat{k}$ estimations, $\mu(\hat{k})$. The standard deviation of those estimates, $\operatorname{std}(\hat{k})$, is an indicative of the estimation quality. If the standard deviation is high, e.g. the following equation is true:

$$
\frac{\mu(\hat{k})+2 \operatorname{std}(\hat{k})}{\mu(\hat{k})} \cdot 100-100>2,
$$

then it is preferable to estimate $k$ and $F_{\text {init }}$ together with the remaining friction parameters, otherwise the prediction may be poor, as the friction parameters may be used to compensate the flaws in this estimation. If it is necessary to estimate $k$ and $F_{\text {init }}$ together with $\theta_{f}$, their search space is:

$$
\begin{aligned}
u b_{s} & =\max \left(1.1, \frac{\mu(k)+2 \operatorname{std}(k)}{\mu(k)}\right) \\
l b_{s} & =\min \left(0.9, \frac{\mu(k)-2 \operatorname{std}(k)}{\mu(k)}\right), \\
u b_{k} & =u b_{s} \cdot \mu(k) \quad l b_{k}=l b_{s} \cdot \mu(k) \\
u b_{F_{\text {init }}} & =u b_{s} \cdot \hat{F}_{\text {init }} \quad l b_{k}=l b_{s} \cdot \hat{F}_{\text {init }},
\end{aligned}
$$


here $u b_{k}$ and $u b_{F_{\text {init }}}$ are the upper bounds of the $k$ and $F_{\text {init }}$ search space, and $l b_{k}$ and $l b_{F_{\text {init }}}$ are its respective lower bounds. Even if the proposed method results in poor estimates, it is still advantageous, since it delivers a search space and an initial guess for $k$ and $F_{\text {init }}$.

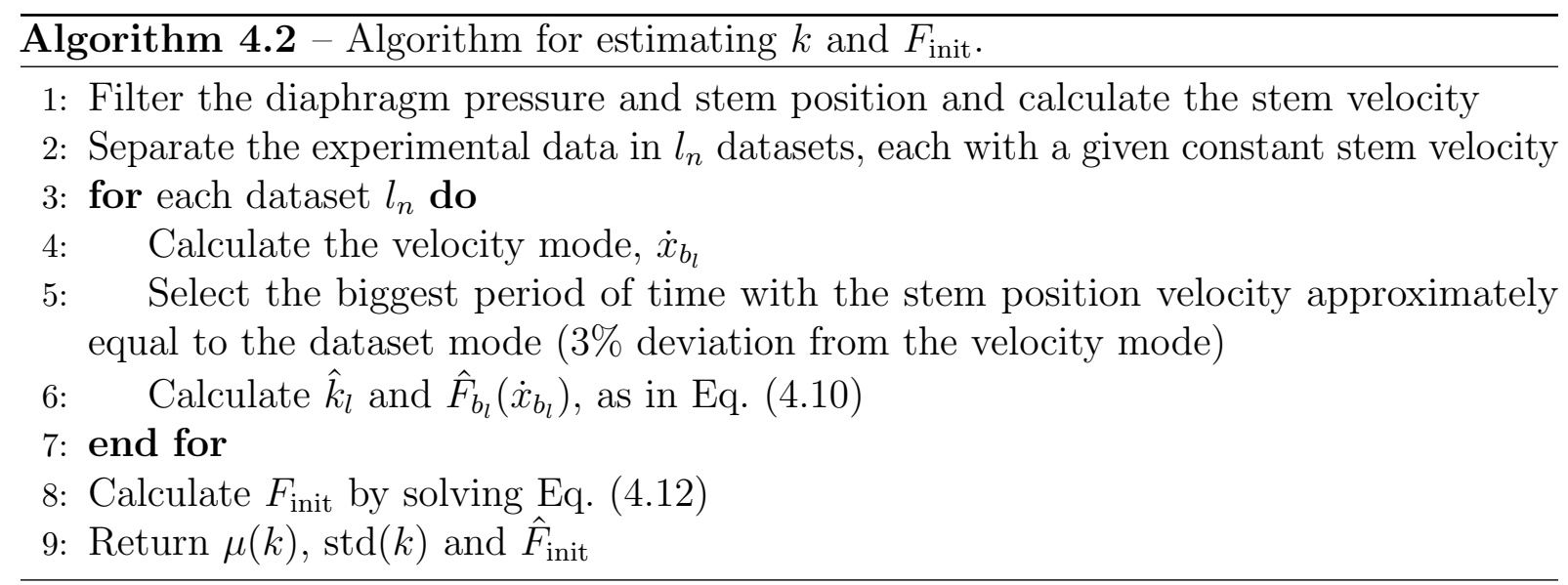

If the Kano model is used instead of estimating $k$ and $F_{\text {init }}$, it is necessary to obtain $x_{\min \mathrm{P}}$ and $x_{\max \mathrm{P}}$, which are easily obtained by obtaining $x$ when $\mathrm{OP}=0$ and $\mathrm{OP}=100$, respectively.

\subsection{Open Loop Friction Parameters Estimation}

Assuming that $S_{0}, \hat{\theta}_{\mathrm{I} / \mathrm{P}}, \hat{k}$ and $\hat{F}_{\text {init }}$ were estimated successfully, it remains to estimate the friction model parameters:

$$
\hat{\theta}_{f}=\underset{\theta_{f}}{\arg \min } V_{n_{f}}\left(S_{a}, m, \hat{k}, \hat{F}_{\text {init }}, \theta_{f}\right)+p\left(\theta_{f}\right)
$$

where $p\left(\theta_{f}\right): \mathbb{R}^{d_{f}} \mapsto \mathbb{R}^{+}$is a penalty function, used when it is necessary to impose parameter constraints. As a reminder, if the a priori estimation of $\hat{k}$ and $\hat{F}_{\text {init }}$ is considered unsatisfactory, then it must be solved Eq. (4.5) instead.

An important aspect of the friction parameters estimation is the excitation signal. It should evidence information related to all friction phenomena discussed in Subsection 3.1.1 (SWEVERS et al., 2000). A sinusoidal velocity signal is able to expose the sliding state characteristics and a ramp signal during presliding may reveal the varying breakaway force and presliding displacement. The presliding hysteresis is neglected purposely in the excitation signal design, since the stem position sensor usually does not have micrometric resolution. The sinusoidal velocity signal is defined as:

$$
v_{s_{j}}(t)=\frac{v_{s_{\max }}-v_{s_{\min }}}{2}+v_{s_{\min }}-\frac{v_{s_{\max }}-v_{s_{\min }}}{2} \cos \left(\frac{2 \pi}{T_{s_{j}}} t\right),
$$

where $v_{s_{j}}(t)$ is the instantaneous velocity of the $j$ th sinusoidal excitation signal, $v_{s_{\max }}$ and $v_{s_{m i n}}$ are the maximum and minimum stem velocities, respectively and $T_{s_{j}}$ is the $j$ th signal 
period. Ideally, it should be generated a series of sinusoidal excitation signals, using high and low velocities and periods. Unfortunately, this is not possible, since the valve has a limited passband and stroke constraints. As the valve is a low-pass system, the minimum period is suggested as:

$$
T_{s_{\min }}=\frac{2 \pi \hat{\tau}_{\mathrm{I} / \mathrm{P}}}{20}
$$

and for the maximum period, to excite the valve with at least half period, one obtains:

$$
T_{s_{\max }}=\frac{2\left(100-S_{0}\right)}{\left(v_{s_{\max }}-v_{s_{\min }}\right) / 2+v_{s_{\min }}} .
$$

The minimum and maximum velocities should be $v_{s_{\min }}<10^{-5} \mathrm{~m} / \mathrm{s}$ and $v_{s_{\max }}>10^{-1}$ $\mathrm{m} / \mathrm{s}$. As there is a trade-off between $v_{s_{\max }}$ and $T_{s_{\max }}$, it is suggested a maximum period of $40 \mathrm{~s}$, since higher periods will not evidence relevant friction characteristics. The ramp signals, with velocities between $v_{s_{\min }}$ and $v_{s_{\max }}$ should be used when the system is in presliding regime until the stem starts moving. Figure 4.4 presents an example of such excitation signal, with 5 different periods $T_{s_{j}}(j=1 \ldots 5)$, and 10 different breakaway velocities (tested in lower and upper stroke). Note that at first, the OP increases rapidly until OP $=0.9 S_{0}$, then the proposed breakaway velocity test is applied until OP $=1.1 S_{0}$. After a short period of stabilization, the sinusoidal velocity signal is applied. Finally, as the velocities and periods span orders of magnitude, it is a good measure to create the test grid using logarithmically spaced points.

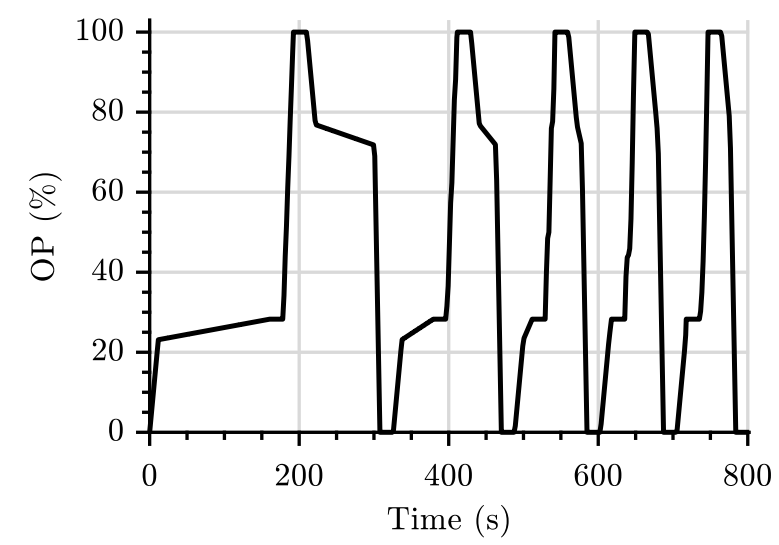

(a) Full signal.

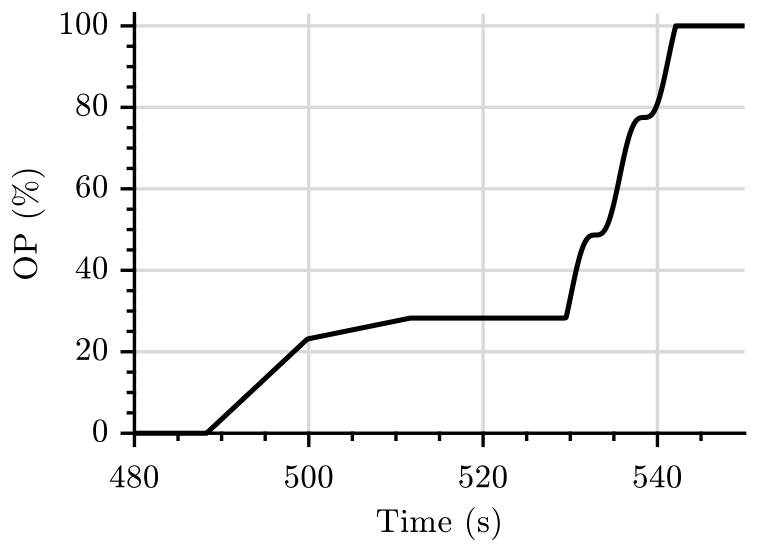

(b) Focus on $480 \mathrm{~s}$ to $550 \mathrm{~s}$.

Figure 4.4 - Example of excitation signal for estimating friction parameters $\left(S_{0} \approx\right.$ $25 \%)$.

Source: from Author.

The parameters search space and constraints are other features that have an impact on the estimation performance. To avoid optimization outside the search space, the following penalty function is used (ARORA, 2015):

$$
p\left(\theta_{f}\right)=\sum_{d=1}^{d_{f}} \max \left(0, l b_{d}-\theta_{f_{d}}\right) \cdot 10^{10}+\max \left(0, \theta_{f_{d}}-u b_{d}\right) \cdot 10^{10}
$$


here the subscript $d$ denotes the selection of the $d$ th element of a vector, lb $\in \mathbb{R}^{d_{f}}$ and $\mathbf{u b} \in \mathbb{R}^{d_{f}}$, which are the lower and upper parameters bounds vector, respectively. Further analysis for defining the search space bounds and eventually other constraints in the search space is carried out for every friction model as follows.

\subsubsection{Kano Search Space Analysis}

As presented in Section 4.1, the valve deadband can be easily estimated. This initial estimation, defined as $S_{0}$, can be used to derive the Kano parameter bounds, as presented in Table 4.1. The slip band is allowed to reach up to $30 \%$ of $S_{0}$ and the initial force can be up to $30 \%$ as well, which is wide enough to model valves with extremely poor performance. If $S_{0}$ is lower than $2 \%$, then it is suggested that the upper bound is calculated considering that $S_{0}$ is $2 \%$ and the lower bound considering that $S_{0}$ is $0 \%$.

Table 4.1 - Kano parameter bounds.

\begin{tabular}{ccc} 
Parameter & $u b_{d}$ & $l b_{d}$ \\
\hline$S$ & $1.3 S_{0}$ & $0.7 S_{0}$ \\
$J$ & $0.3 S_{0}$ & 0 \\
$D$ & 30 & 0 \\
\hline
\end{tabular}

\subsubsection{Karnopp Search Space Analysis}

Given $S_{0}$, it is possible to calculate $F_{s_{0}}$, by rearranging Eq. (3.31):

$$
F_{s_{0}}=\left(P_{\max }-P_{\min }\right) \cdot S_{a} \cdot\left(S_{0} / 200\right) .
$$

It is assumed that the maximum absolute estimation error of $F_{s_{0}}$ is at most $30 \%$.

The Coulomb friction parameter is derived by noting that $F_{c} \leq F_{s}$ and assuming that $F_{c} \geq 0.7 F_{s}$.

As the viscous friction parameter may span a wide range, it is suggested to deliver its upper bound as a hundred times higher than $F_{s_{0}}$ with maximum stem velocity, calculated as presented in Eq. (3.28). The lower bound is derived similarly, by assuming that the viscous friction is a hundredth of $F_{s_{0}}$ at the maximum stem velocity.

The Stribeck velocity parameter search space should span from $10^{-5}$ to $10^{-1} \mathrm{~m} / \mathrm{s}$, which are the limits obtained from experimental data, as presented in Subsection 3.1.1. Since the $v_{s}$ search space is wide, it may decrease the optimization technique performance. An experimental evidence that can reduce the search space is the stick-slip behavior when exciting the valve with certain constant velocities. Consider the linear approximation, for $\dot{x}>0$, of the valve with Karnopp friction model in the Laplace domain:

$$
\frac{\delta x(s)}{\delta P(s)}=\frac{S_{a}}{s^{2}+H_{k}\left(\dot{x}_{0}\right) s+k / m},
$$


here $\delta$ denotes an incremental variable, e.g. $\delta x(t)=x(t)-x_{0}, x_{0}$ is a linearization point, and $H_{k}\left(\dot{x}_{0}\right)$ is the first derivative of the Stribeck curve with respect to $\dot{x}$, divided by $m$ :

$$
H_{k}\left(\dot{x}_{0}\right)=\frac{1}{m}\left[F_{v}-2 \cdot\left(F_{s}-F_{c}\right) \mathrm{e}^{-\left(\dot{x}_{0} / v_{s}\right)^{2}} \frac{\dot{x}_{0}}{v_{s}^{2}}\right],
$$

where $\dot{x}_{0}$ is the linearization velocity. What can be concluded from Eqs. (4.23) and (4.24) is that the Stribeck effect decreases the system damping. If the velocity is such that the damping is lower than 1, then the linear approximation presents an overshoot, which corresponds to a velocity reversal, switching the valve to the presliding state, leading to a stick-slip behavior. The system damping is calculated as:

$$
\xi_{K}(\dot{x})=\frac{H_{k}(\dot{x})}{2 \sqrt{m k}}
$$

where $\xi_{K}$ is the valve with Karnopp friction model damping.

To obtain the velocity in which occurs the largest damping decrease, it is necessary to obtain the derivative of Eq. (4.24) and equaling to zero:

$$
\begin{aligned}
0 & =-\left(F_{s}-F_{c}\right) \mathrm{e}^{-\left(\dot{x}_{0 k} / v_{s}\right)^{2}}\left(\frac{2}{v_{s}^{2}}-\frac{4 \dot{x}_{0}^{2}}{v_{s}^{4}}\right), \\
\dot{x}_{0_{p k}} & =v_{s} / \sqrt{2}
\end{aligned}
$$

where $\dot{x}_{0_{p k}}$ is the highest decrease in the system damping. If the stick-slip behavior is detected for some velocity range, being $v_{s l_{1}}$ and $v_{s l_{2}}$ the lower and upper bounds of this range, then the Stribeck velocity is certainly $v_{s} \in\left[v_{s l_{1}} / 10,10 v_{s l_{2}}\right]$. If no stick-slip behavior is detected, then it indicates that $F_{s}$ is close to $F_{c}$, or $F_{v}$ is much higher than any decrease in the system damping, or the Stribeck velocity is higher than the stem velocities tested.

As an example, consider a valve with the same parameters as in Tables 3.1 and 3.2, except for $F_{s}=800 \mathrm{~N}$ and $F_{v}=50000 \mathrm{Ns} / \mathrm{m}$. Figure 4.5 presents the behavior of $\xi_{K}$ for given stem velocities. Note that the predicted stick-slip behavior is within $\left[6 \cdot 10^{-5}, 8 \cdot 10^{-4}\right]$ $\mathrm{m} / \mathrm{s}$.

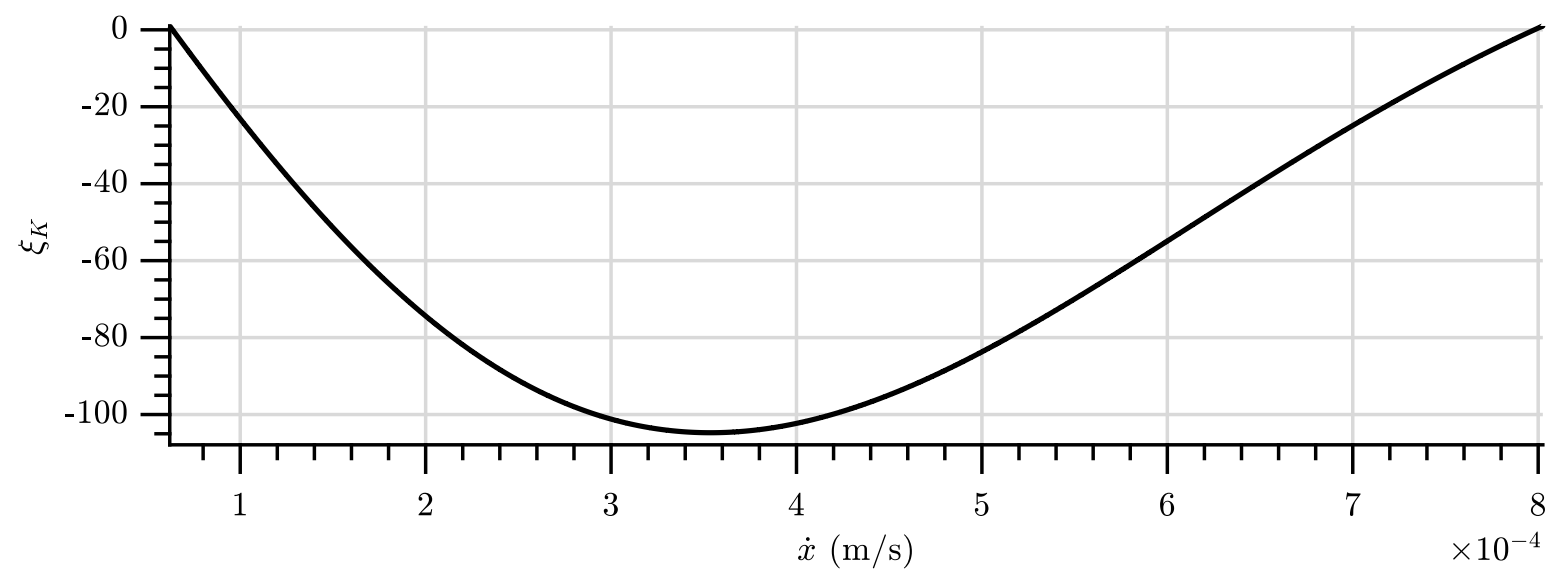

Figure $4.5-\xi_{K}$ variation with respect to the stem velocity, for $v_{s}=5 \cdot 10^{-4} \mathrm{~m} / \mathrm{s}$. Source: from Author. 
The system simulation is presented in Figures 4.6 to 4.8. The tested velocities are: $10^{-6}, 1.56 \cdot 10^{-6}, 2.43 \cdot 10^{-6}, 3.80 \cdot 10^{-6}, 5.92 \cdot 10^{-6}, 9.24 \cdot 10^{-6}, 1.44 \cdot 10^{-5}, 2.25 \cdot 10^{-5}$, $3.51 \cdot 10^{-5}, 5.47 \cdot 10^{-5}, 8.54 \cdot 10^{-5}, 1.33 \cdot 10^{-4}, 2.08 \cdot 10^{-4}, 3.24 \cdot 10^{-4}, 5.06 \cdot 10^{-4}$ and $7.89 \cdot 10^{-4}$ $\mathrm{m} / \mathrm{s}$. The stick-slip prediction is correct for low velocities, but it must be pointed out that given the I/P bandwidth, it was not possible to test higher stem velocities. A better visualization of the stick-slip behavior can be seen in Figure 4.8.

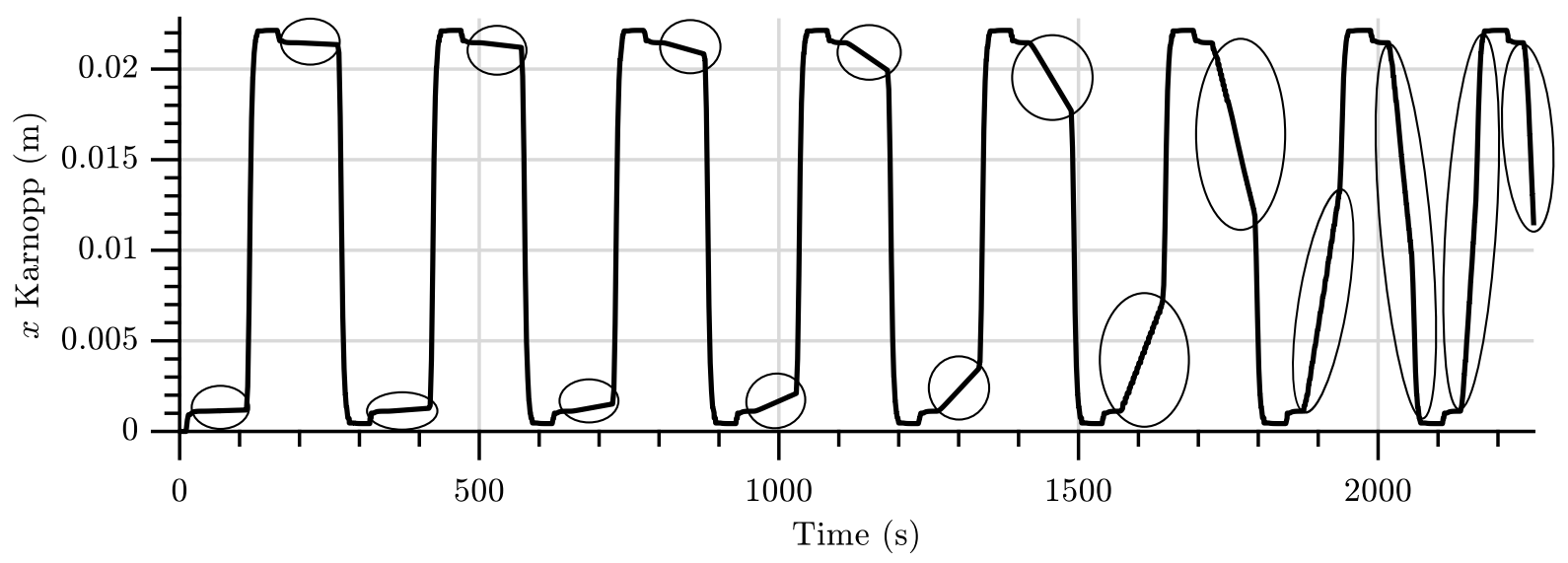

Figure 4.6 - Stick-slip stem position behavior simulation using valve with the Karnopp model.

Source: from Author.

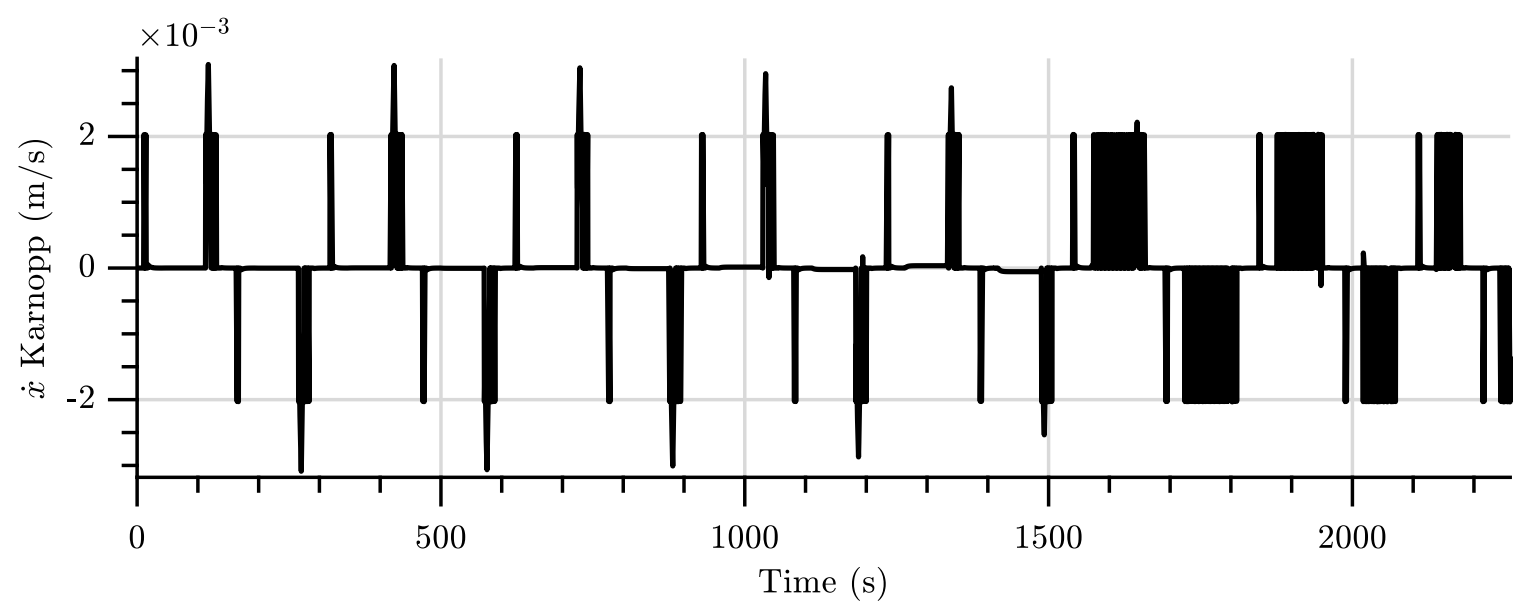

Figure 4.7 - Stick-slip stem velocity behavior simulation using valve with the Karnopp model.

Source: from Author. 


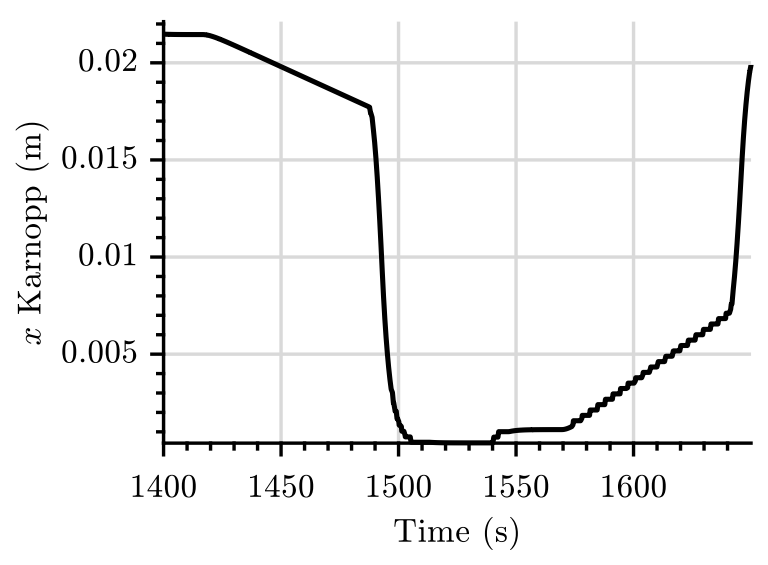

(a) Stem position.

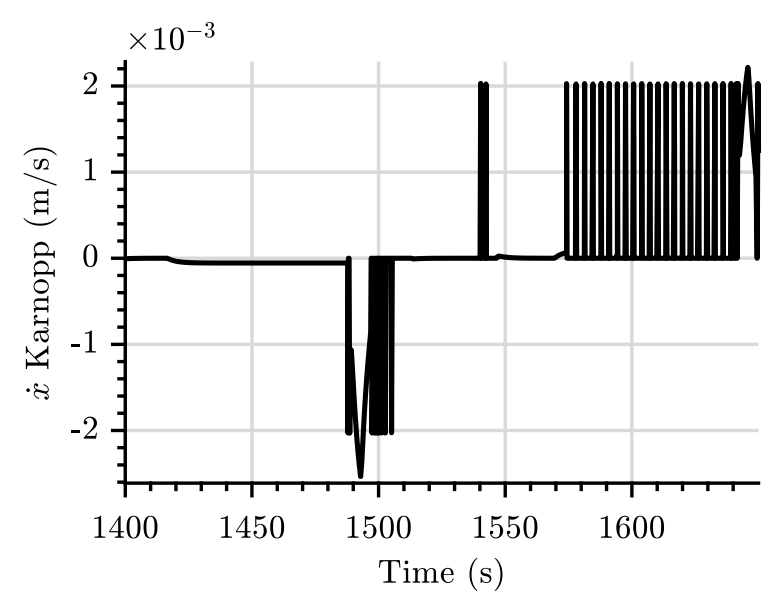

(b) Stem velocity.

Figure 4.8 - Stick-slip behavior simulation, with focus in excitation signals of $5.47 \cdot 10^{-5} \mathrm{~m} / \mathrm{s}$ and $8.54 \cdot 10^{-5} \mathrm{~m} / \mathrm{s}$.

Source: from Author.

This analysis is an approximation of the physical behavior, as the model complexity was simplified by a linearization and the Karnopp model itself neglects many friction characteristics and the I/P model is more complex. However, some stick-slip behavior can be seen in the experimental data of the graphite gasket valve, as presented in Figures 4.9 to 4.12 .

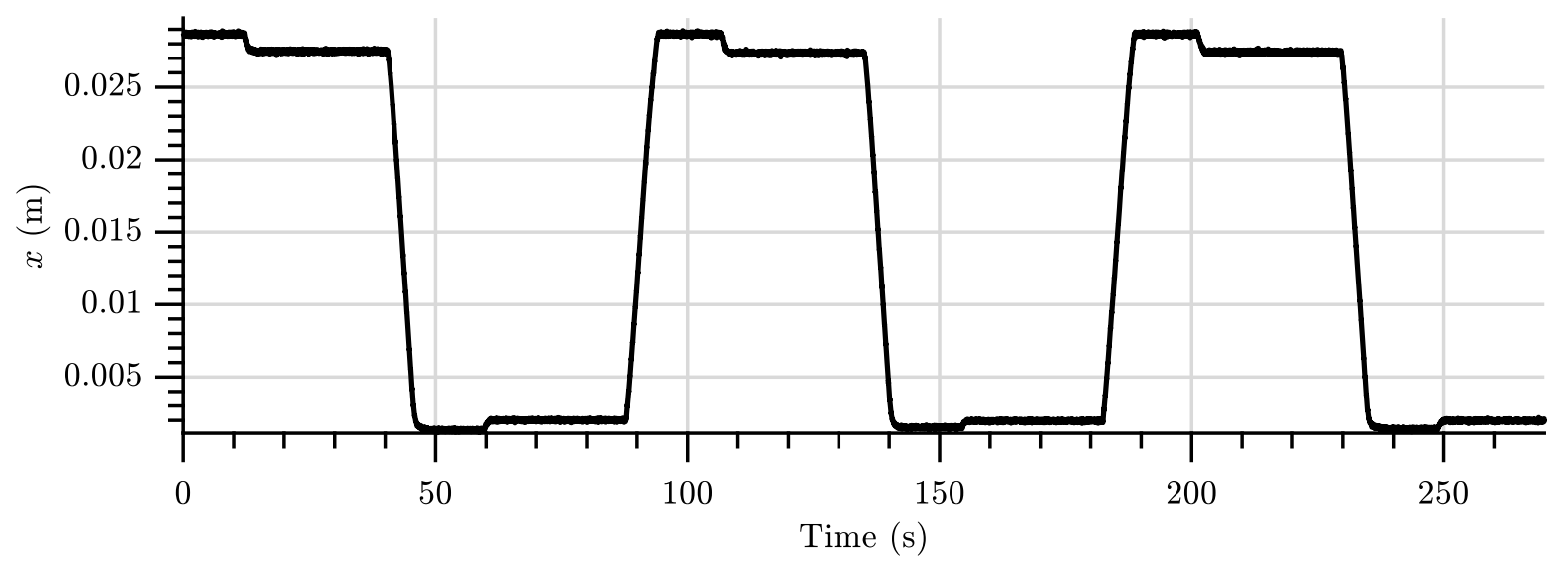

Figure 4.9 - No stick-slip from velocities lower than $1.26 \cdot 10^{-4} \mathrm{~m} / \mathrm{s}$. Source: from Author. 


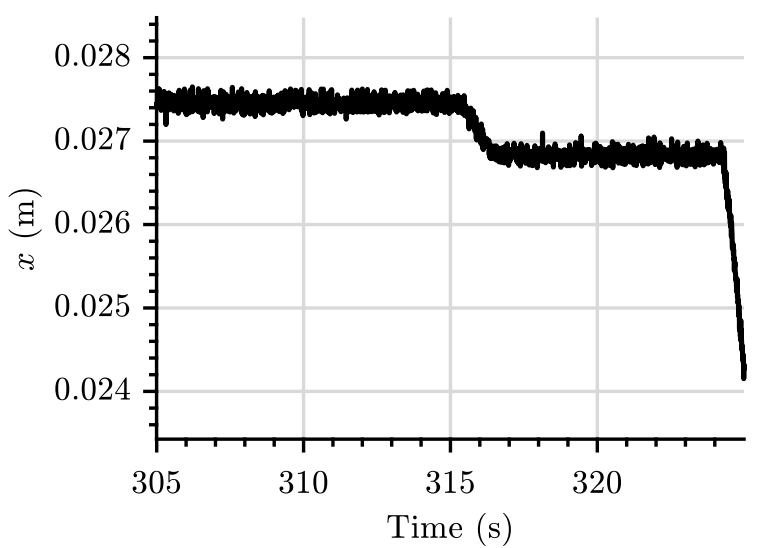

(a) A slip with $2.16 \cdot 10^{-4} \mathrm{~m} / \mathrm{s}$ excitation signal.

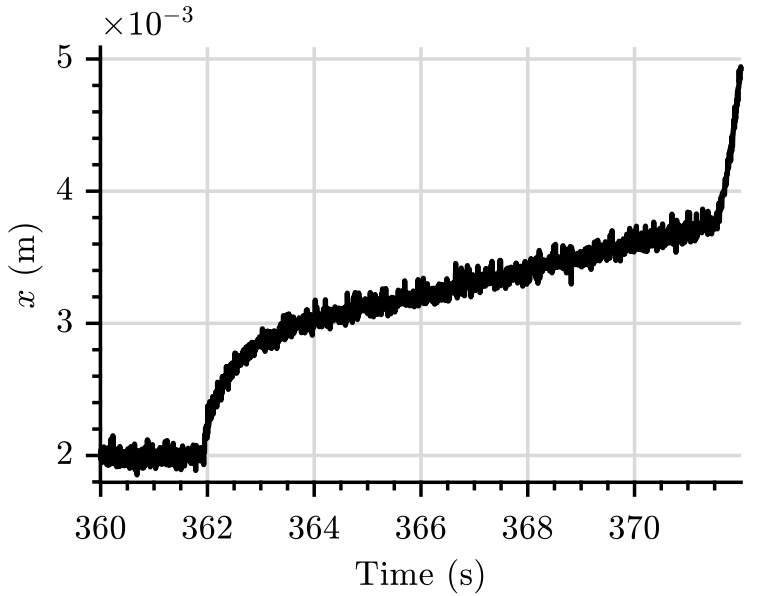

(b) A slip with $3.70 \cdot 10^{-4} \mathrm{~m} / \mathrm{s}$ excitation signal.

Figure 4.10 - Slip phenomenon found at certain velocities.

Source: from Author.

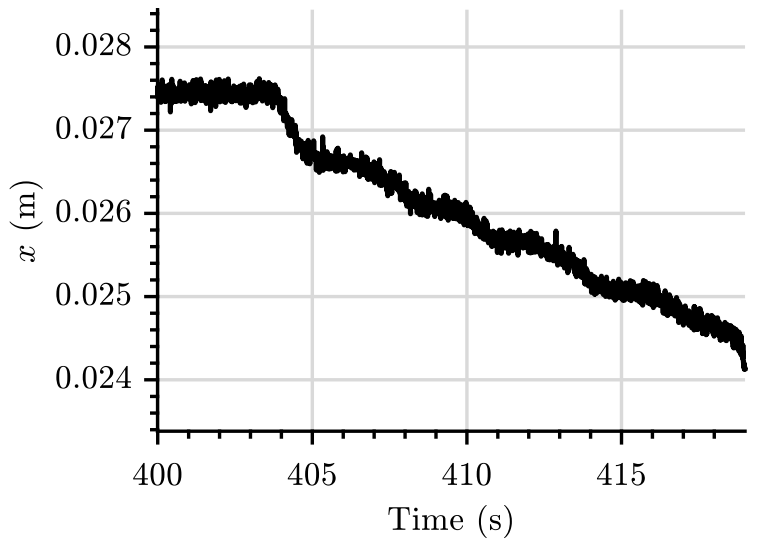

(a) A stick-slip behavior with $6.33 \cdot 10^{-4} \mathrm{~m} / \mathrm{s}$ excitation signal.

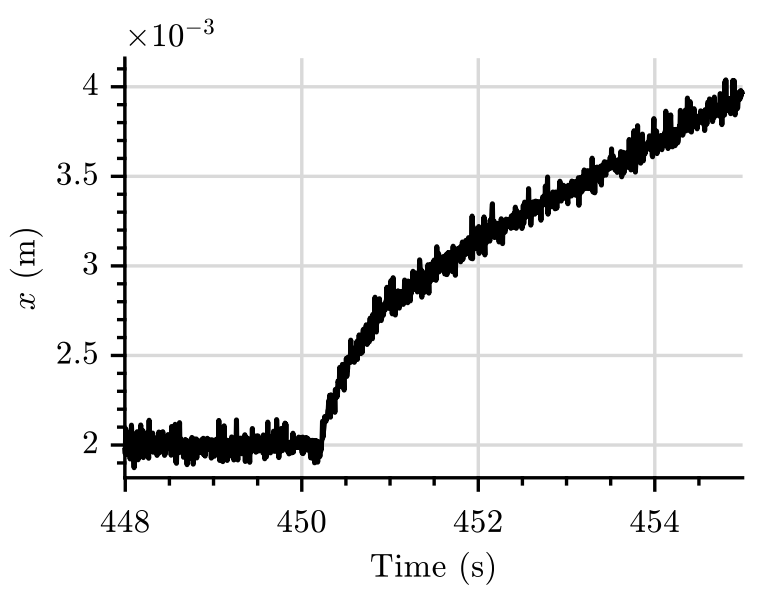

(b) A slip with $1.08 \cdot 10^{-3} \mathrm{~m} / \mathrm{s}$ excitation signal.

Figure 4.11 - Slip and stick-slip phenomenon found at certain velocities. Source: from Author.

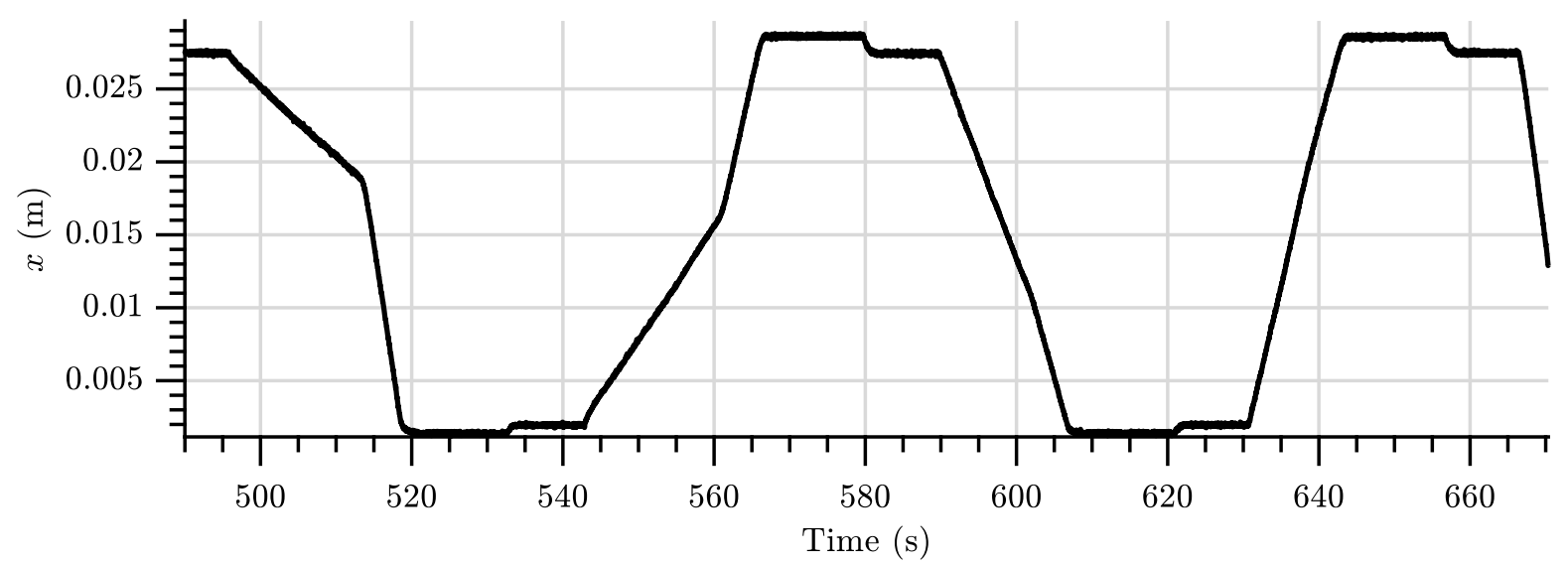

Figure 4.12 - No stick-slip from velocities higher than $1.86 \cdot 10^{-3} \mathrm{~m} / \mathrm{s}$. Source: from Author. 
A generic search space for the Karnopp friction parameters is presented in Table 4.2. The $v_{s}$ search space range can be smaller if any stick-slip behavior is detected, however the safest choice is to search the entire proposed $v_{s}$ space, as detecting stick-slip behavior without visual inspection is a difficult task. If $S_{0}$ is lower than $2 \%$, then it is suggested that the upper and lower bounds of $F_{c}, F_{s}$ and $F_{v}$ are calculated assuming that $S_{0}$ is $2 \%$ and $0 \%$, respectively.

Table 4.2 - Karnopp parameter bounds.

\begin{tabular}{ccc} 
Parameter & $u b_{d}$ & $l b_{d}$ \\
\hline$F_{c}$ & $1.3 F_{s_{0}}$ & $0.49 F_{s_{0}}$ \\
$F_{s}$ & $1.3 F_{s_{0}}$ & $0.7 F_{s_{0}}$ \\
$F_{v}$ & $100 \cdot F_{s_{0}} / v_{\max }$ & $\left(F_{s_{0}} / 100\right) / v_{\max }$ \\
$v_{s}$ & $10^{-1}$ & $10^{-5}$ \\
\hline \multicolumn{3}{c}{ Source: from Author }
\end{tabular}

To avoid the case $F_{c}>F_{s}$, Eq. (4.21) is augmented:

$$
p_{k}\left(\theta_{f}\right)=p\left(\theta_{f}\right)+\max \left(0, \quad \theta_{f_{1}}-\theta_{f_{2}}\right) \cdot 10^{10},
$$

where $\theta_{f_{1}}=F_{c}, \theta_{f_{2}}=F_{s}$ and $p_{k}\left(\theta_{f}\right)$ is the penalty function for Karnopp friction parameters estimation.

\subsubsection{LuGre Search Space Analysis}

As the LuGre model is also based on the Stribeck curve, the Karnopp search space derived in the previous subsection can be readily applied. However, the LuGre has some additional parameters, one to control the presliding displacement $\left(\sigma_{0}\right)$ and the other controls the friction damping $\left(\sigma_{1}\right)$, which is able to modify the overall system damping, possibly avoiding the stick-slip behavior even with a high difference between $F_{s}$ and $F_{c}$. This occurs because $\dot{z}$ is positive when the velocity is reducing towards $0^{+} \mathrm{m} / \mathrm{s}$, increasing the system damping.

Olsson et al. (1998) suggest that it is possible to obtain an estimate of the system damping by linearizing around $\dot{x}=0^{+}$and $z=0^{+}$. At this point, the linear approximation of the valve model, Eq. (3.1), with the LuGre friction model, Eqs. (3.9) and (3.10), in Laplace domain, are given by:

$$
S_{a} \delta P(s)=m s^{2} \delta x(s)+\left(F_{v}+\sigma_{1}\right) s \delta x(s)+\left(k+\sigma_{0}\right) \delta x(s) .
$$

Therefore, one can derive the system damping:

$$
\xi_{L}=\frac{\sigma_{1}+F_{v}}{2 \sqrt{m\left(k+\sigma_{0}\right)}},
$$


where $\xi_{L}$ is the valve with LuGre friction model damping. Thus, if stick-slip behavior is detected when exciting the valve with a ramp signal, not only information regarding $v_{s}$ is given, but also may indicate that $\sigma_{1}<2 \sqrt{m\left(k+\sigma_{0}\right)}-F_{v}$. However, this equation may not be taken as correct, given the simplifications applied in the analysis.

An example can be seen in Figures 4.13 and 4.14, where a valve with LuGre friction model was simulated with the parameters of Tables 3.1 and 3.2 , except for: $F_{s}=800 \mathrm{~N}$, $F_{v}=50000 \mathrm{Ns} / \mathrm{m}, \sigma_{0}=10^{6} \mathrm{~N} / \mathrm{m}$ and $\sigma_{1}=2 \cdot 10^{3} \mathrm{Ns} / \mathrm{m}$, which corresponds to the same Stribeck curve parameters where stick-slip behavior is present in the valve with Karnopp friction model. However, $\sigma_{1}$ is high enough to prevent the stick-slip behavior in the LuGre model.

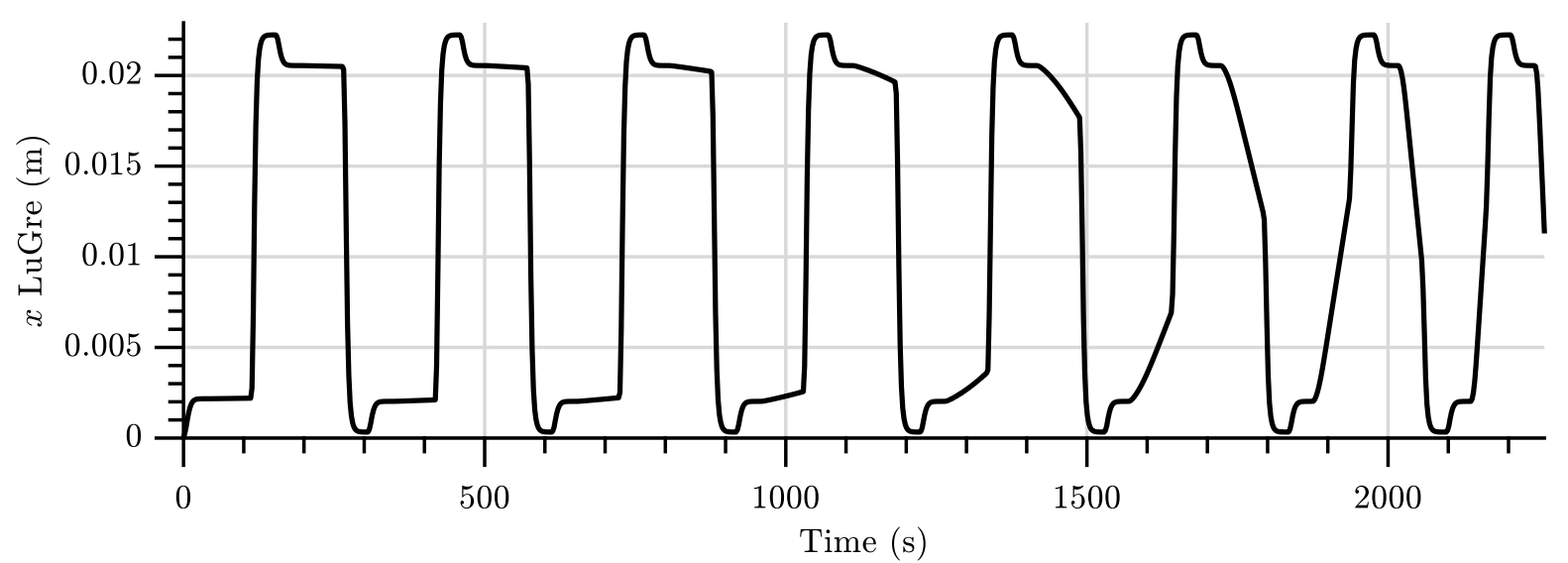

Figure 4.13 - Valve with LuGre model stem position simulation, with no stick-slip behavior.

Source: from Author.

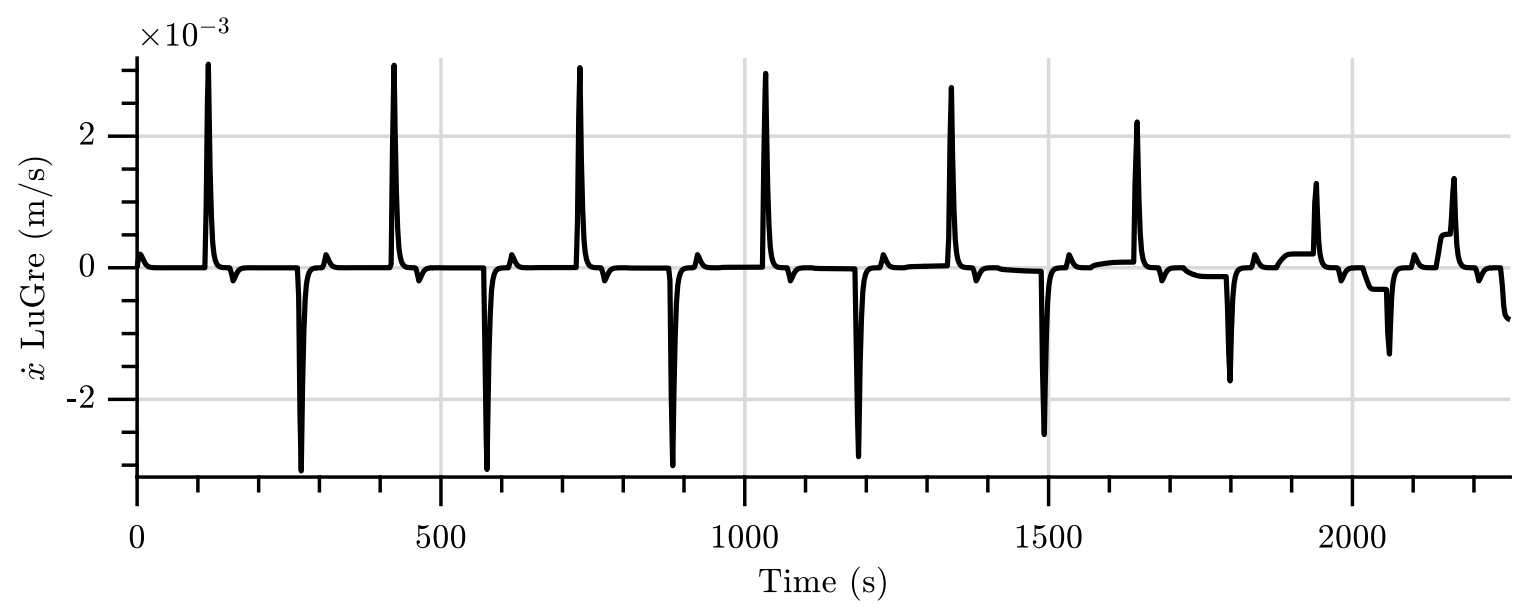

Figure 4.14 - Valve with LuGre model stem velocity simulation, with no stick-slip behavior.

Source: from Author.

The generic search space is composed by the same values of Table 4.2, extended by the parameter bounds presented in Table 4.3. The assumption is that the minimum 
and the maximum presliding displacement are 1 and $50 \mu \mathrm{m}$, respectively, when friction is equal to $F_{s}$. The friction damping parameter is allowed to reach high values, to model a wide range of experimental data. The penalty function is the same developed for the Karnopp model, as shown in Eq. (4.27).

Table 4.3 - LuGre parameter bounds, an extension of Table 4.2.

\begin{tabular}{ccc} 
Parameter & $u b_{d}$ & $l b_{d}$ \\
\hline$\sigma_{0}$ & $1.3 F_{s_{0}} \cdot 10^{6}$ & $0.014 F_{s_{0}} \cdot 10^{6}$ \\
$\sigma_{1}$ & $0.014 F_{s_{0}} \cdot 10^{6}$ & 500 \\
\hline
\end{tabular}

Another issue with the LuGre model is determining the initial state of its internal variable $z$. In the open loop test, the valve always start from the lowermost position and to reach that state, $z$ has to be at its minimum position, therefore:

$$
z[0]=-\frac{F_{s}}{\sigma_{0}} .
$$

\subsubsection{GMS Search Space Analysis}

Once more, the Karnopp search space can be used for the GMS model, as it is based on the Stribeck curve as well. In addition, the presliding displacement and friction damping for each massless Maxwell element is controlled by $\kappa_{i}$ and $\nu_{i}$, respectively. Yet, $\alpha_{i}$ determines how each Maxwell element contributes to the observed friction force and $C$ defines the velocity to friction lag. Similarly to the LuGre model, the valve damping may be increased by the parameters $\nu_{i}$.

The valve damping, in the presliding state, can be derived using the valve model, Eq. (3.1), and the presliding GMS model, given by Eqs. (3.12) and (3.13):

$$
\xi_{G}=\frac{F_{v}+\sum_{i=1}^{N_{m_{e}}} \nu_{i}}{2 \sqrt{m\left(k+\sum_{i=1}^{N_{m_{e}}} \kappa_{i}\right)}},
$$

where $\xi_{G}$ is the valve with GMS model damping. Therefore, if stick-slip behavior is present, then it may be an indicator that $\sum_{i=1}^{N_{m_{e}}} \nu_{i}<2 \sqrt{m\left(k+\sum_{i=1}^{N_{m_{e}}} \kappa_{i}\right)}-F_{v}$, as well as imply the approximate value of $v_{s}$, as already discussed in Subsection 4.2.2.

As an example of how it is possible to control the stick-slip behavior with $\nu$, a valve with GMS friction model was simulated using the same parameters of Tables 3.1 and 3.2 , except for: $F_{s}=800 \mathrm{~N}$ and $F_{v}=50000 \mathrm{Ns} / \mathrm{m}$. The results are presented in Figures 4.15 and 4.16, where despite the Stribeck parameters resulted in stick-slip behavior when the valve was simulated with Karnopp friction model, the stick-slip was not present in the GMS simulation due to $\nu$ additional damping. 


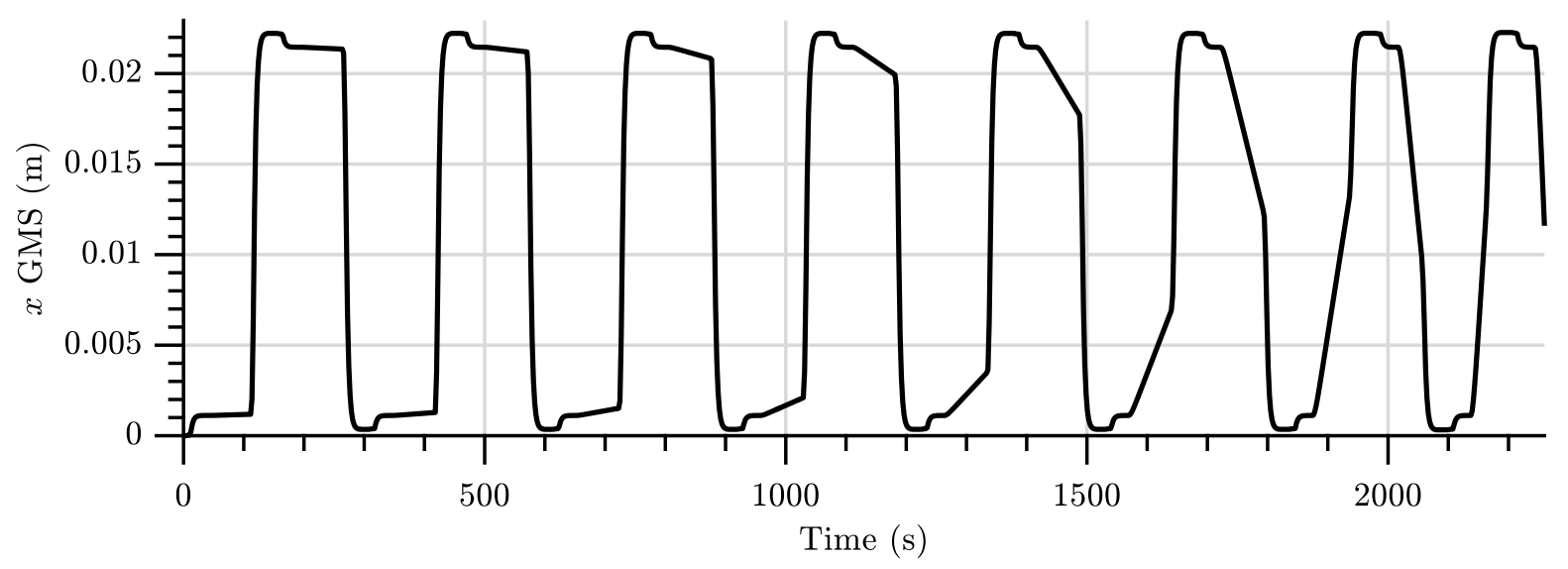

Figure 4.15 - Valve with GMS model stem position simulation, with no stick-slip behavior.

Source: from Author.

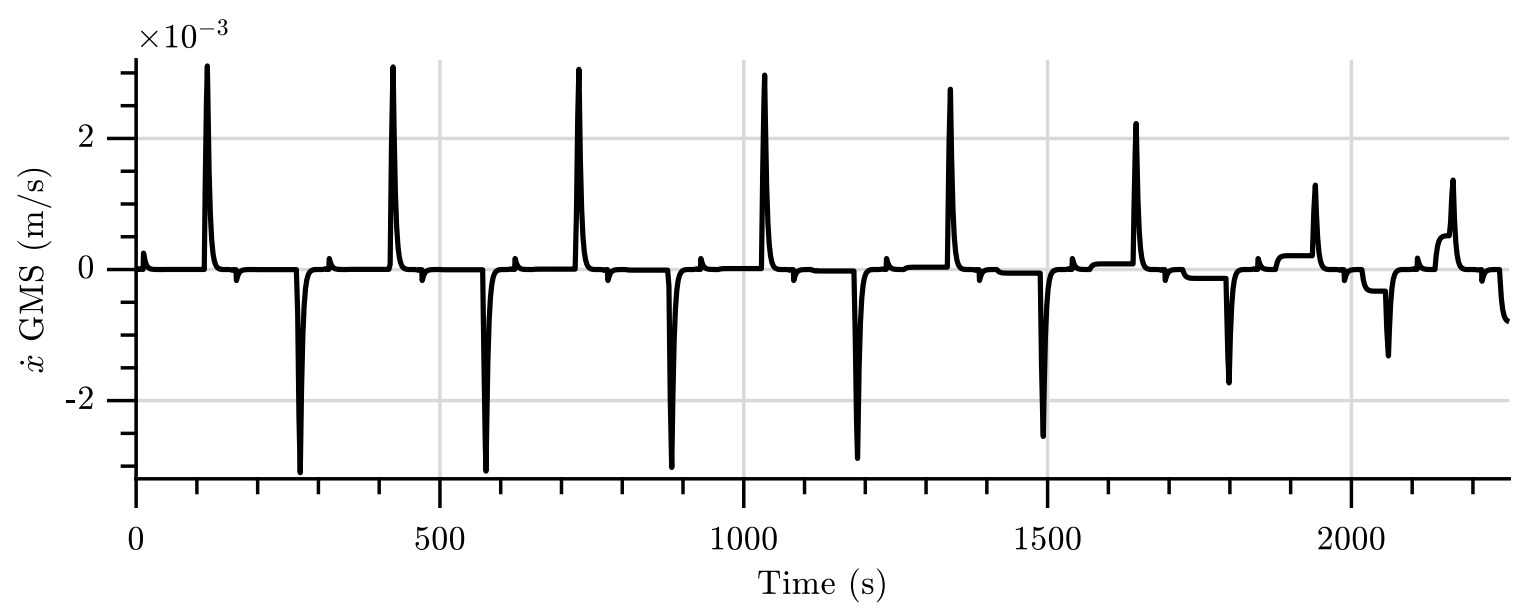

Figure 4.16 - Valve with GMS model stem velocity simulation, with no stick-slip behavior.

Source: from Author.

The general GMS parameter search space, presented in Table 4.4, is an extension of the Karnopp bounds presented in Table 4.2. The premises used to derive the GMS $\kappa_{i}$ and $\nu_{i}$ bounds are the same used for $\sigma_{0}$ and $\sigma_{1}$ definition in the LuGre model, respectively. The bounds for $C$ are derived assuming that the velocity to friction lag may vary from 1 ms to 0.1 s (ARMSTRONG-HÉLOUVRY; DUPONT; CANUDAS de WIT, 1994).

Table 4.4 - GMS parameter bounds, an extension of Table 4.2.

\begin{tabular}{ccc} 
Parameter & $u b_{d}$ & $l b_{d}$ \\
\hline$\kappa_{i}$ & $1.3 F_{s_{0}} \cdot 10^{6}$ & $0.014 F_{s_{0}} \cdot 10^{6}$ \\
$\nu_{i}$ & $0.014 F_{s_{0}} \cdot 10^{6}$ & 500 \\
$\alpha_{i}$ & 1 & 0 \\
$C$ & $10^{3}$ & 10 \\
\hline
\end{tabular}


The penalty function used to estimate the GMS parameters is an extension of Eq. (4.27), to guarantee that $\sum_{i=1}^{N_{m}} \alpha_{i}=1$ :

$$
p_{g}\left(\theta_{f}\right)=p_{k}\left(\theta_{f}\right)+\max \left(0, \quad\left|\left(\sum_{i=1}^{N_{m_{e}}} \alpha_{i}\right)-1\right|\right) \cdot 10^{10}
$$

where $p_{g}\left(\theta_{f}\right)$ is the penalty function for the GMS friction parameters estimation.

For determining the initial internal states, $\psi$, the same reasoning used in the LuGre model is applied to the GMS model, therefore:

$$
\psi_{i}[0]=-\alpha_{i} \frac{F_{s}}{\kappa_{i}} .
$$

\subsubsection{Optimization Method Selection}

The friction models are nonlinear and discontinuous, which imposes difficulties for applying optimization methods that are based on the objective function differentiation, e.g. Steepest Descent or Newton-Raphson, as demonstrated by Marques and Garcia (2019). Therefore, the best option is to use a derivative free method with a fair capacity to avoid local minima. For that, some optimization techniques were tested to support the selection of a proper estimator.

This test is performed using the valve with LuGre friction model only, since it is the one with intermediate complexity between the models proposed in this thesis. The tests consists in identifying the friction parameters of a simulated valve model, without any measurement noise and with a perfect knowledge of $m, k, S_{a}, F_{\text {init }}, x_{\min }$ and $x_{\max }$. Also, there is a perfect knowledge of $\tau_{\mathrm{I} / \mathrm{P}}$, which is used only to design the sinusoidal velocity excitation signal. The term $S_{0}$ was estimated previously using the Algorithm 4.1 and the search space is calculated as presented. The valve and friction parameters are the same shown in Tables 3.1 and 3.2. In summary, this test is an ideal case, with a perfect match between plant and model, without any measurement noise and a perfect knowledge of the valve parameters.

In the first three tests, three different residual function calculations were tested. The first is Mean Squared Error (MSE), as in Eq. (4.17). The second is the Integral Absolute Error (IAE), defined as:

$$
V_{a_{n_{f}}}\left(\theta_{f}\right)=\sum_{n=1}^{n_{f}}\left|e_{x}\left(n, \theta_{f}\right)\right|
$$

The third is the Integral Root Absolute Error (IRAE), defined as:

$$
V_{r_{n_{f}}}\left(\theta_{f}\right)=\sum_{n=1}^{n_{f}} \sqrt{\left|e_{x}\left(n, \theta_{f}\right)\right|}
$$


The mean squared error function maps the errors in a number using a quadratic equation, which amplifies or attenuates errors higher or lower than 1, respectively. On the other hand, the integral absolute error just adds the errors without any modification. Finally, the squared absolute residual has the reverse effect of the mean squared error function. The difference is numerical only, as all three functions will increase if any error occur, but each will do it with a different gain.

Some optimization techniques fail when the parameters differ many orders of magnitude, which is exactly the case in this thesis. Therefore, in the first three tested optimization algorithms, the parameters normalization, using the parameter lower and upper bounds, is tested.

\subsubsection{Genetic Algorithm Optimization (GA)}

The Genetic Algorithm is based on species evolution characteristics. The basic idea is to generate a random population of parameter vectors and to apply some evolutionary functions to the individuals (parameter vectors) to approximate the optimization problem solution. The scheme used in this thesis is presented in Algorithm A.1, from Appendix A, which is based on Ferragud (1999).

The results using the Genetic Algorithm optimization are presented in Tables 4.5 and 4.6. By analyzing the results, it is possible to conclude that the prediction performance is approximately the same, no matter what residual function is used or if the parameter normalization is performed or not. Another finding is that even with enormous errors in $\hat{v}_{s}$ and $\hat{\sigma}_{1}$, the overall friction prediction is quite good. This demonstrates that some parameters have low influence on the error function, making it difficult to estimate these parameters.

Table 4.5 - GA optimization LuGre parameter estimation results.

\begin{tabular}{llrrr} 
Function & Norm. & fit (\%) & Run Time (h) & Func. Evals. \\
\hline MSE & No & 99.50 & 42.25 & 43860 \\
IAE & No & 99.47 & 33.93 & 36120 \\
IRAE & No & 99.49 & 33.61 & 36120 \\
MSE & Yes & 99.50 & 36.42 & 37800 \\
IAE & Yes & 99.46 & 23.10 & 24780 \\
IRAE & Yes & 99.42 & 22.50 & 24120 \\
\hline
\end{tabular}

Norm.: indicates if the parameters are normalized (Yes) or not (No) and Func. Evals.: indicates to the number of objective function evaluations.

Source: from Author. 
Table 4.6 - GA optimization LuGre parameter estimation error.

\begin{tabular}{llrrrrrr} 
Residuals & Norm. & $\hat{F}_{c}(\%)$ & $\hat{F}_{s}(\%)$ & $\hat{F}_{v}(\%)$ & $\hat{v}_{s}(\%)$ & $\hat{\sigma}_{0}(\%)$ & $\hat{\sigma}_{1}(\%)$ \\
\hline MSE & No & 11.16 & -0.20 & -38.88 & $1.82 \cdot 10^{4}$ & -12.51 & $1.97 \cdot 10^{2}$ \\
IAE & No & 9.58 & 0.07 & -42.65 & $1.98 \cdot 10^{4}$ & 0.53 & -88.11 \\
IRAE & No & 11.33 & 0.06 & -40.47 & $1.97 \cdot 10^{4}$ & 1.20 & -97.29 \\
MSE & Yes & 10.85 & -0.32 & -38.14 & $1.84 \cdot 10^{4}$ & -14.01 & -97.55 \\
IAE & Yes & -5.54 & 0.08 & -43.93 & $1.78 \cdot 10^{4}$ & 0.94 & $4.36 \cdot 10^{2}$ \\
IRAE & Yes & 9.82 & 0.07 & -46.67 & $1.69 \cdot 10^{4}$ & 0.50 & $6.31 \cdot 10^{2}$ \\
\hline
\end{tabular}

Source: from Author.

\subsubsection{Differential Evolution Optimization (DE)}

The differential evolution algorithm is based on generating a population of random parameters vectors and applying aleatory linear combinations on aleatory population members. The Algorithm A.2 from Appendix A summarizes the method, which is proposed by Storn and Price (1997).

The estimation results using DE algorithm are presented in Tables 4.7 and 4.8. It is possible to conclude that the $f i t$ and the estimation error performance is similar to the GA algorithm, however, the number of function evaluations and thus the run time, are significantly lower.

Table 4.7 - DE optimization LuGre parameters estimation results.

\begin{tabular}{llrrr} 
Residuals & Norm. & fit $(\%)$ & Run Time $(\mathrm{h})$ & Func. Evals. \\
\hline MSE & No & 99.50 & 11.30 & 12121 \\
IAE & No & 99.47 & 11.48 & 12361 \\
IRAE & No & 99.46 & 13.79 & 14881 \\
MSE & Yes & 99.50 & 11.28 & 12121 \\
IAE & Yes & 99.47 & 11.26 & 12121 \\
IRAE & Yes & 99.46 & 12.18 & 13141 \\
\hline
\end{tabular}

Norm.: indicates if the parameters are normalized (Yes) or not (No) and Func. Evals.: refers to the number of objective function evaluations.

Source: from Author.

Table 4.8 - DE optimization LuGre parameters estimation error.

\begin{tabular}{llrrrrrr} 
Residuals & Norm. & $\hat{F}_{c}(\%)$ & $\hat{F}_{s}(\%)$ & $\hat{F}_{v}(\%)$ & $\hat{v}_{s}(\%)$ & $\hat{\sigma}_{0}(\%)$ & $\hat{\sigma}_{1}(\%)$ \\
\hline MSE & No & 11.13 & -0.27 & -37.96 & $1.86 \cdot 10^{4}$ & -9.99 & -97.53 \\
IAE & No & 11.50 & 0.06 & -42.55 & $1.81 \cdot 10^{4}$ & 0.47 & $5.09 \cdot 10^{2}$ \\
IRAE & No & -29.75 & 0.07 & -44.07 & $1.83 \cdot 10^{4}$ & 0.02 & $6.38 \cdot 10^{2}$ \\
MSE & Yes & 11.13 & -0.27 & -37.96 & $1.92 \cdot 10^{4}$ & -9.98 & -97.49 \\
IAE & Yes & 11.50 & 0.06 & -42.56 & $1.93 \cdot 10^{4}$ & 0.47 & $5.10 \cdot 10^{2}$ \\
IRAE & Yes & -20.51 & 0.07 & -44.10 & $1.99 \cdot 10^{4}$ & 0.15 & $6.28 \cdot 10^{2}$ \\
\hline \multicolumn{7}{c}{ Source: from Author. }
\end{tabular}




\subsubsection{Monte Carlo with Simplex Optimization (MS)}

As the estimator is failing due to local minima, as already seen in previous tests, it was selected a Monte Carlo method together with a Simplex optimization, which is synthesized in Algorithms A.3 and A.4, from Appendix A. This algorithm is based on selecting many aleatory points in the search space and evaluating its residuals. Then, the points with the best residuals are used as the starting point of a Simplex optimization.

The results are presented in Tables 4.9 and 4.10. This algorithm performance is better than the previous ones, specially when the MSE residual function is used, regardless of the parameter normalization. However, $\sigma_{1}$ estimation errors are still high. It is clear, though, that the best residual function choice until now is the MSE, which is the only used in succeeding tests. As no difference is perceived whether the parameters are normalized or not, future tests are performed only with non normalized parameters.

Table 4.9 - MS optimization LuGre parameter estimation results.

\begin{tabular}{llrrr} 
Residuals & Norm. & fit $(\%)$ & Run Time $(\mathrm{h})$ & Func. Evals. \\
\hline MSE & No & 100.00 & 13.88 & 12986 \\
IAE & No & 99.47 & 14.80 & 13843 \\
IRAE & No & 99.46 & 9.52 & 9073 \\
MSE & Yes & 100.00 & 13.71 & 12843 \\
IAE & Yes & 99.47 & 13.40 & 12613 \\
IRAE & Yes & 99.46 & 6.00 & 5953 \\
\hline
\end{tabular}

Norm.: indicates if the parameters are normalized (Yes) or not (No) and Func. Evals.: refers to the number of objective function evaluations.

Source: from Author.

Table 4.10 - MS optimization LuGre parameters estimation error.

\begin{tabular}{llrrrrrr} 
Residuals & Norm. & $\hat{F}_{c}(\%)$ & $\hat{F}_{s}(\%)$ & $\hat{F}_{v}(\%)$ & $\hat{v}_{s}(\%)$ & $\hat{\sigma}_{0}(\%)$ & $\hat{\sigma}_{1}(\%)$ \\
\hline MSE & No & 0.00 & 0.00 & 0.00 & -0.01 & 0.05 & -99.85 \\
IAE & No & 11.35 & 0.06 & -42.56 & $1.88 \cdot 10^{4}$ & 0.48 & $1.85 \cdot 10^{2}$ \\
IRAE & No & 10.32 & 0.07 & -44.00 & $4.21 \cdot 10^{3}$ & 0.35 & -96.66 \\
MSE & Yes & -0.02 & 0.00 & 0.04 & 0.23 & -0.36 & $1.28 \cdot 10^{2}$ \\
IAE & Yes & 10.80 & 0.06 & -42.58 & $1.71 \cdot 10^{4}$ & 0.51 & -82.20 \\
IRAE & Yes & -19.47 & 0.05 & -35.31 & $9.36 \cdot 10^{3}$ & 0.25 & 34.48 \\
\hline \multicolumn{7}{c}{ Source: from Author. }
\end{tabular}

\subsubsection{Successive Nonlinear Linear Regression (SNL)}

This method is suggested by Rizos and Fassois (2009), which consists in separating the optimization problem in a linear part and a nonlinear part to estimate the LuGre parameters. The difference from the proposed algorithm is that $F_{f}$ is not a measured variable, which imposes higher difficulties in estimating the parameters, which has to be done with $x$ only. The algorithm scheme is presented in Algorithm A.5 from Appendix A. 
The friction force can be calculated by rearranging Eq. (3.1):

$$
F_{f}[n]=S_{a} P[n]-\hat{k} x[n]-\hat{F}_{\text {init }}-\hat{m} \hat{\ddot{x}}[n],
$$

where $\hat{\ddot{x}}$ is the estimated valve acceleration, using backward differencing of the measured stem position. Note that no noise is present in this test. The algorithm starts by testing aleatory points in the search space. The best residual from the previous step is used as the starting point. Then, the optimization cycle is initialized by optimizing the nonlinear parameters, $F_{c}, F_{s}$ and $v_{s}$ using GA, with fixed linear parameters, $F_{v}, \sigma_{0}$ and $\sigma_{1}$. With the new nonlinear parameters, a valve with LuGre model is simulated and by noting that:

$$
F_{f}[n]=\sigma_{0} \hat{z}[n]+\sigma_{1} \hat{\dot{z}}[n]+F_{v} \hat{\dot{x}}[n]
$$

the linear parameters are easily estimated using LSE. The cycle is then restarted and continues until the finishing criteria is met.

This algorithm results are presented in Table 4.11 and 4.12 . The results are quite similar to the GA and DE algorithms, which is not surprising, as this algorithm uses the GA to estimate the nonlinear parameters. One explanation for this inability to find the residual function minimum is an inherent difficulty, due to the low parameter sensitivity and local minima, and the problem separation, which lowers the search dimension, is not enough to overcome the problem. Despite this algorithm can perform better if the estimator parameters are tuned, this algorithm is not suitable for practical applications, since the friction force calculation would be imprecise, due to measurement noise and indirect variables calculation.

Table 4.11 - SNL optimization LuGre parameter estimation results.

\begin{tabular}{ccc} 
fit $(\%)$ & Run Time $(\mathrm{h})$ & Func. Evals. \\
\hline 99.50 & 19.43 & 18281 \\
\hline \multicolumn{3}{c}{ Source: from Author. }
\end{tabular}

Func. Evals.: refers to the number of objective function evaluations.

Table 4.12 - SNL optimization LuGre parameter estimation error.

\begin{tabular}{cccccc}
$\hat{F}_{c}(\%)$ & $\hat{F}_{s}(\%)$ & $\hat{F}_{v}(\%)$ & $\hat{v}_{s}(\%)$ & $\hat{\sigma}_{0}(\%)$ & $\hat{\sigma}_{1}(\%)$ \\
\hline 11.11 & -0.28 & -37.72 & $1.97 \cdot 10^{4}$ & -10.22 & -97.55 \\
\hline \multicolumn{6}{c}{ Source: from Author. }
\end{tabular}

\subsubsection{Grid Search with Differential Evolution and Simplex Optimization (GDES)}

The first stage of this algorithm is an initial evaluation of the search space, which is done with by selecting just a few points for every parameter, since a more refined search would lead to computationally impossible problems. The best residuals are then used as an input for a DE optimization, which result is used for a further Simplex optimization, for a 
more precise estimation of the minima. This algorithm scheme is presented in Algorithm A.6 from Appendix A.

As this is the selected algorithm for estimating the friction parameters, all models are tested, and the results are shown in Tables 4.13 to 4.17. The Kano, Karnopp and LuGre models presented a perfect $f i t$ index, even though the $\sigma_{1}$ LuGre parameter is estimated with an error of $1 \%$. The GMS model parameters, however, were estimated with high errors, specially for $\kappa, \nu$ and $\alpha$. Even though there were high errors in the estimations, the fit is notably high, evidencing the high complexity in estimating the GMS parameters, as its sensitivities are low to the overall valve stem position prediction. Despite the errors regarding the GMS model, this is the algorithm with the best performance so far and it is selected as the estimator, since trying to refine the GMS estimations would lead to eventually a change in the GMS model, to improve identifiability or to use an estimator that would result in far more function evaluations than this estimator.

Table 4.13 - GDES estimation results.

\begin{tabular}{lrrr} 
Model & fit $(\%)$ & Run Time $(\mathrm{h})$ & Func. Evals. \\
\hline Kano & 100.00 & 0.06 & 4585 \\
Karnopp & 100.00 & 14.53 & 16058 \\
LuGre & 100.00 & 39.40 & 45107 \\
GMS & 99.97 & 225.53 & 133575 \\
\hline \multicolumn{4}{c}{ Source: from Author. }
\end{tabular}

Func. Evals.: refers to the number of objective function evaluations.

Table 4.14 - GDES Kano parameter estimation error.

\begin{tabular}{ccc}
$\hat{S}(\%)$ & $\hat{J}(\%)$ & $\hat{D}(\%)$ \\
\hline 0.00 & 0.00 & 0.00 \\
\hline \multicolumn{3}{c}{ Source: from Author. }
\end{tabular}

Table 4.15 - GDES Karnopp parameter estimation error.

\begin{tabular}{cccc}
$\hat{F}_{c}(\%)$ & $\hat{F}_{s}(\%)$ & $\hat{F}_{v}(\%)$ & $\hat{v}_{s}(\%)$ \\
\hline 0.00 & 0.00 & 0.00 & 0.02 \\
\hline \multicolumn{4}{c}{ Source: from Author. }
\end{tabular}

Table 4.16 - GDES LuGre parameter estimation error.

\begin{tabular}{cccccc}
$\hat{F}_{c}(\%)$ & $\hat{F}_{s}(\%)$ & $\hat{F}_{v}(\%)$ & $\hat{v}_{s}(\%)$ & $\hat{\sigma}_{0}(\%)$ & $\hat{\sigma}_{1}(\%)$ \\
\hline 0.00 & 0.00 & 0.00 & 0.00 & 0.00 & 1.01 \\
\hline \multicolumn{6}{c}{ Source: from Author. }
\end{tabular}


Table 4.17 - GDES GMS parameter estimation error.

\begin{tabular}{ccccccc}
$\hat{F}_{c}(\%)$ & $\hat{F}_{s}(\%)$ & $\hat{F}_{v}(\%)$ & $\hat{v}_{s}(\%)$ & $\hat{\kappa}_{1}(\%)$ & $\hat{\kappa}_{2}(\%)$ & $\hat{\kappa}_{3}(\%)$ \\
\hline 1.45 & 0.00 & 0.36 & -4.63 & 52.06 & 25.18 & $2.64 \cdot 10^{3}$ \\
\hline$\hat{\nu}_{1}(\%)$ & $\hat{\nu}_{2}(\%)$ & $\hat{\nu}_{3}(\%)$ & $\hat{\alpha}_{1}(\%)$ & $\hat{\alpha}_{2}(\%)$ & $\hat{\alpha}_{3}(\%)$ & $\hat{C}(\%)$ \\
\hline $3.77 \cdot 10^{3}$ & $6.59 \cdot 10^{2}$ & $3.71 \cdot 10^{3}$ & -31.06 & $1.40 \cdot 10^{2}$ & -94.34 & 23.03 \\
\hline
\end{tabular}

Source: from Author.

\subsection{Simulated Tests}

Some tests were performed to guarantee that the proposed open loop parameter estimation method is feasible under influence of measurement noise. In this test, the plant is the simulated valves with parameters presented in Tables 3.1 and 3.2, but with a noise addition that results in a $25 \mathrm{~dB}$ (signal mean around 316 times higher than the noise standard deviation) Signal to Noise Ratio (SNR) in measured variables $(x$ and $P$ ).

To estimate $S_{0}$, the test is performed using the valve with Karnopp friction model, and it is performed exactly as described in Section 4.1. The diaphragm pressure and stem position used in this test are presented in Figure 4.17.

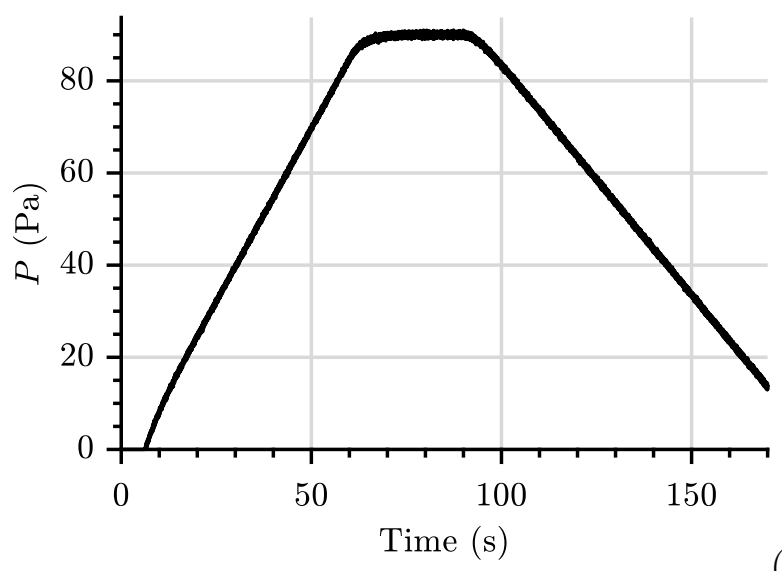

(a) Normalized diaphragm pressure.

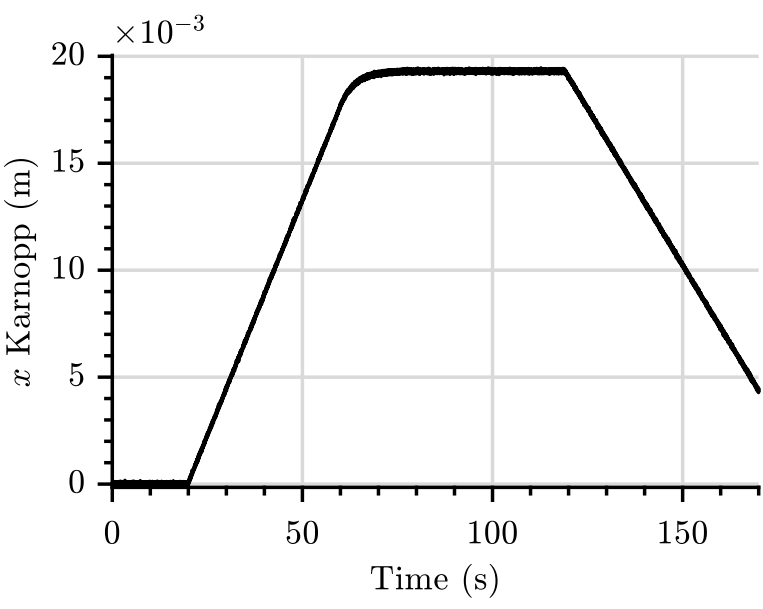

(b) Valve stem position given by valve with Karnopp friction model.

Figure 4.17 - Signals used for testing the $S_{0}$ estimation algorithm.

Source: from Author.

Analogously, the I/P converter parameters are estimated using the excitation signal and algorithm proposed in Section 4.1. The test signals are presented in Figure 4.18. 


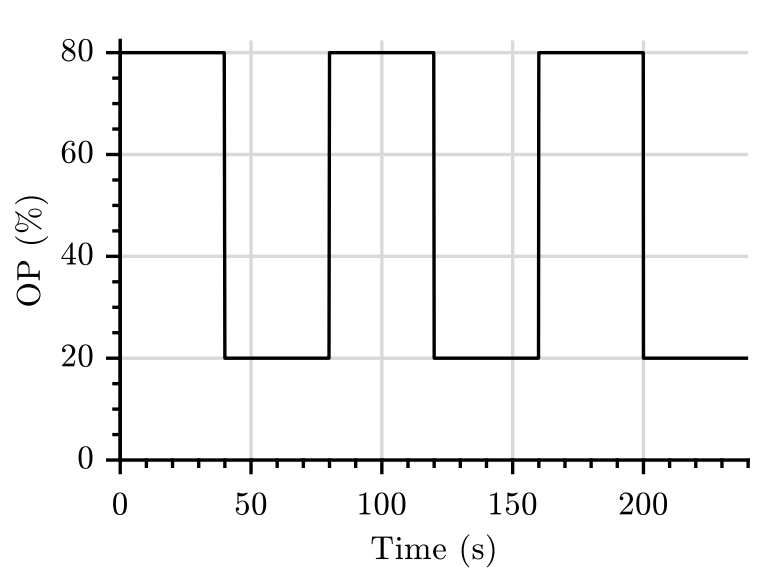

(a) Controller output.

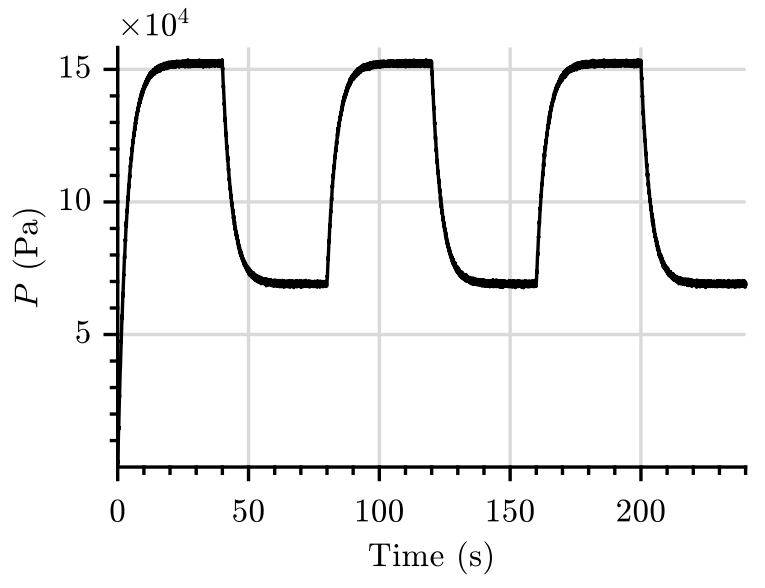

(b) Diaphragm pressure.

Figure 4.18 - Signals used for testing the I/P parameter estimation algorithm.

Source: from Author.

To estimate $k$ and $F_{\text {init }}$, the valve with Karnopp friction model is used. Again, the test is performed exactly as suggested in Section 4.1 and the signals used in the estimation are presented in Figure 4.19.

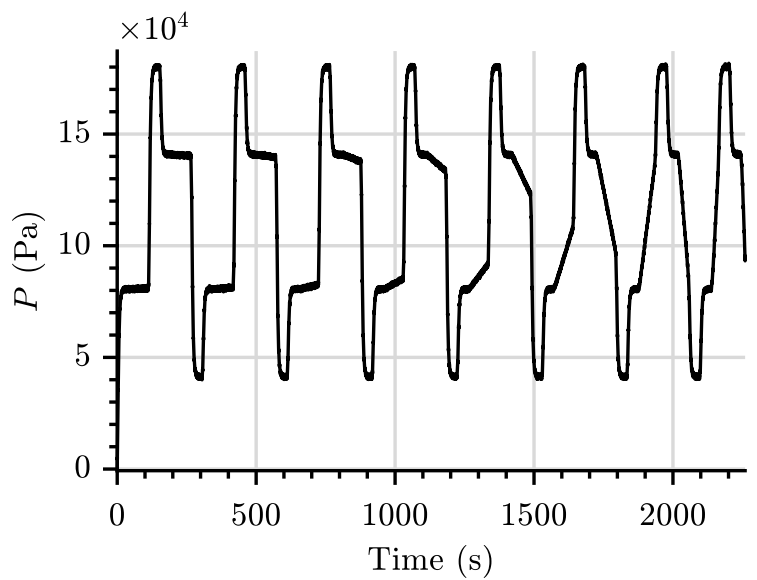

(a) Diaphragm pressure.

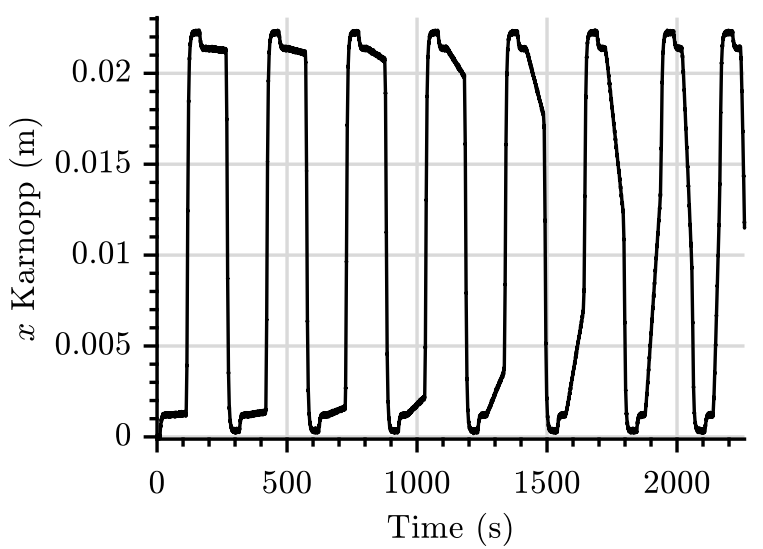

(b) Valve stem position given by valve with Karnopp friction model.

Figure 4.19 - Signals used for testing the $k$ and $F_{\text {init }}$ estimation algorithm.

Source: from Author.

The results are presented in Table 4.18. For comparison purposes, the algorithms were run in a case without measurement noise, as well. It is possible to verify that the results are very consistent, with a slightly increase in the estimation error as the noise increases. The $S_{0}$ algorithm has an inherent error, which is necessary to avoid incorrect stem movement detection due to measurement noise. 
Table 4.18 - Open loop valve parameters estimation error in simulated case.

\begin{tabular}{lrr} 
Parameter & $\begin{array}{r}\text { Without noise } \\
\text { est. error (\%) }\end{array}$ & $\begin{array}{r}\text { 25dB SNR } \\
\text { est. error }(\%)\end{array}$ \\
\hline$S_{0}$ & 0.97 & 0.97 \\
$\hat{P}_{\min }$ & -0.01 & -0.02 \\
$\hat{K}_{P_{g}}$ & -0.02 & -0.01 \\
$\hat{\tau}_{\mathrm{I} / \mathrm{P}}$ & 0.01 & -0.07 \\
$\hat{k}$ & 0.00 & -0.01 \\
$\hat{F}_{\text {init }}$ & -0.01 & 0.03 \\
\hline
\end{tabular}

As for the friction estimation test, the GDES estimator was used with non normalized parameters. The excitation signal and the search space were designed according Section 4.2 guidelines. The diaphragm pressure and stem position are filtered using a zero phase second order linear filter with 0.9 relative damping and $100 / \tau_{\mathrm{I} / \mathrm{P}}$ natural frequency. Additionally, the diaphragm pressure is filtered using a Kalman filter with a plant model as in Eq. (3.20), measurement variance estimated by the difference between simulated and measured diaphragm pressure during the I/P converter parameter estimation algorithm and the model variance assumed to be 50 times lower than the measurement variance. The Kalman filter is defined as:

$$
\begin{aligned}
L_{p}[n] & =M[n-1]-\frac{M[n-1]^{2}}{M[n-1]+\sigma_{\text {meas }}^{2}} \\
L_{c}[n] & =\frac{L_{p}[n]}{\sigma_{\text {meas }}} \\
P_{\text {priori }}[n] & =P_{\text {filt }}[n-1]+L_{c}[n]\left(\bar{P}[n-1]-P_{\text {filt }}[n-1]\right) \\
P_{\text {filt }}[n] & =\hat{a}_{0} P_{\text {priori }}[n]+\hat{b}_{1} \mathrm{OP}[n-1] \\
M[n] & =\hat{a}_{0}^{2} \cdot L_{p}[n]+\sigma_{\text {mod }}^{2} \quad \text { with } \quad M[0]=1,
\end{aligned}
$$

where $\bar{P}[n]=P[n]-P_{\min }$, is the incremental diaphragm pressure, $\sigma_{\text {meas }}^{2}$ is the measurement variance, $\sigma_{\text {mod }}^{2}$ is the model variance and $P_{\text {filt }}[n]$ is the Kalman filtered diaphragm pressure. The estimation results are presented in Tables 4.19 to 4.23 .

Table 4.19 - Friction parameter estimation test with simulated data results.

\begin{tabular}{lrrr} 
Model & fit $(\%)$ & Run Time $(\mathrm{h})$ & Func. Evals. \\
\hline Kano & 99.26 & 0.09 & 8010 \\
Karnopp & 99.99 & 17.54 & 19322 \\
LuGre & 99.92 & 34.01 & 39167 \\
GMS & 99.98 & 182.48 & 112240 \\
\hline
\end{tabular}

Source: from Author.

Func. Evals.: refers to the number of objective function evaluations. 
Table 4.20 - Kano parameter estimation error, test with simulated data.

\begin{tabular}{ccc}
$\hat{S}(\%)$ & $\hat{J}(\%)$ & $\hat{D}(\%)$ \\
\hline-2.62 & -57.16 & 0.15 \\
\hline \multicolumn{3}{c}{ Source: from Author. }
\end{tabular}

Table 4.21 - Karnopp parameter estimation error, test with simulated data.

\begin{tabular}{cccc}
$\hat{F}_{c}(\%)$ & $\hat{F}_{s}(\%)$ & $\hat{F}_{v}(\%)$ & $\hat{v}_{s}(\%)$ \\
\hline 0.04 & 0.02 & -0.30 & 0.12 \\
\hline \multicolumn{4}{c}{ Source: from Author. }
\end{tabular}

Table 4.22 - LuGre parameter estimation error, test with simulated data.

\begin{tabular}{cccccc}
$\hat{F}_{c}(\%)$ & $\hat{F}_{s}(\%)$ & $\hat{F}_{v}(\%)$ & $\hat{v}_{s}(\%)$ & $\hat{\sigma}_{0}(\%)$ & $\hat{\sigma}_{1}(\%)$ \\
\hline 0.10 & 0.19 & -0.48 & -1.86 & 24.27 & $3.88 \cdot 10^{2}$ \\
\hline \multicolumn{6}{c}{ Source: from Author. }
\end{tabular}

Table 4.23 - GMS parameter estimation error, test with simulated data.

\begin{tabular}{ccccccc}
$\hat{F}_{c}(\%)$ & $\hat{F}_{s}(\%)$ & $\hat{F}_{v}(\%)$ & $\hat{v}_{s}(\%)$ & $\hat{\kappa}_{1}(\%)$ & $\hat{\kappa}_{2}(\%)$ & $\hat{\kappa}_{3}(\%)$ \\
\hline-2.79 & 0.02 & -0.51 & 6.59 & -10.71 & 23.65 & $2.23 \cdot 10^{2}$ \\
\hline$\hat{\nu}_{1}(\%)$ & $\hat{\nu}_{2}(\%)$ & $\hat{\nu}_{3}(\%)$ & $\hat{\alpha}_{1}(\%)$ & $\hat{\alpha}_{2}(\%)$ & $\hat{\alpha}_{3}(\%)$ & $\hat{C}(\%)$ \\
\hline $2.30 \cdot 10^{3}$ & $2.78 \cdot 10^{3}$ & $1.00 \cdot 10^{2}$ & -5.68 & 46.30 & -99.98 & -26.68 \\
\hline \multicolumn{7}{c}{ Source: from Author. }
\end{tabular}

The Kano model is highly affected by measurement noise, presenting significantly higher errors when compared to the ideal case test. The main reason for that is because this model lacks any dynamics, responding to any frequency, even for small measurement noises. Had the pressure not been filtered, the results would be worst, as presented in Figure 4.20.

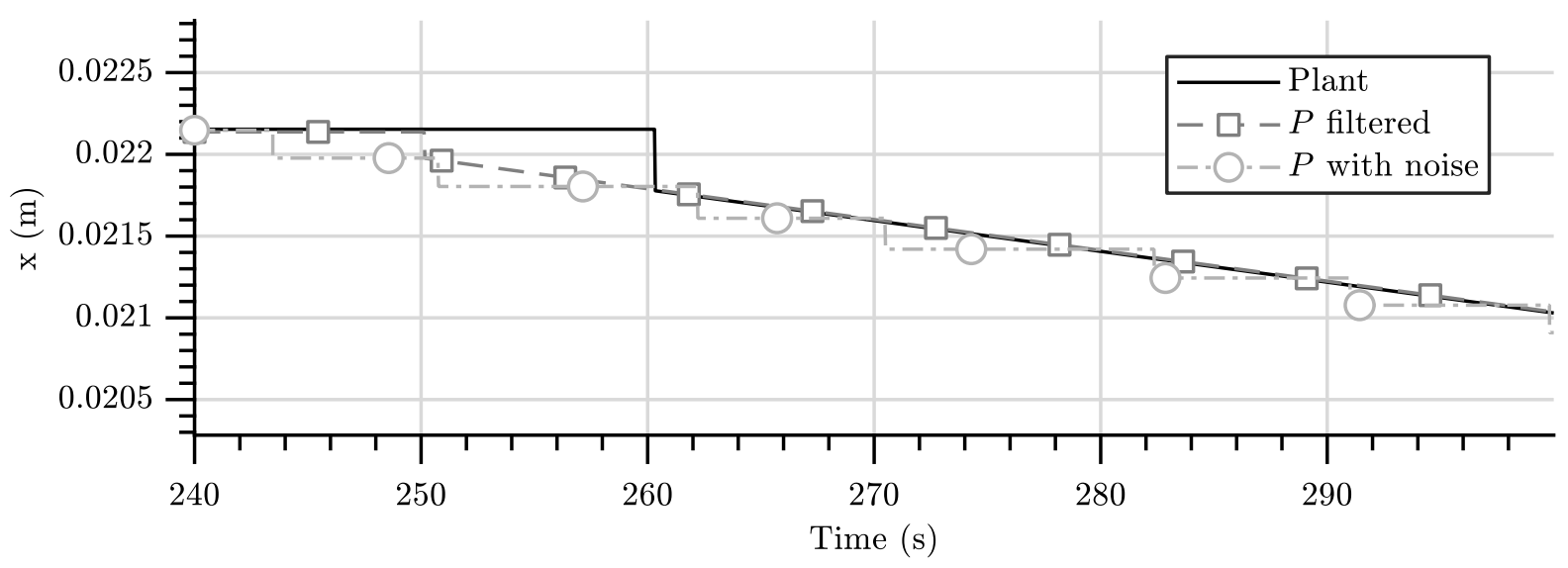

Figure 4.20 - Deterioration of Kano model performance due to noise.

Source: from Author. 
Excellent performance is hold by the Karnopp model, even in the presence of measurement noise. The LuGre model still presents a great performance, but the noise resulted in poorer estimates for $\sigma_{0}$ and $\sigma_{1}$, which did not affect considerably the fit index. One practical aspect that is worth mentioning is that the LuGre model imprecision regarding presliding displacement may lead to a drift in the system position, which is not physically plausible, as can be seen in Figure 4.21. This problem is not present in GMS models, as shown in Figure 4.22.

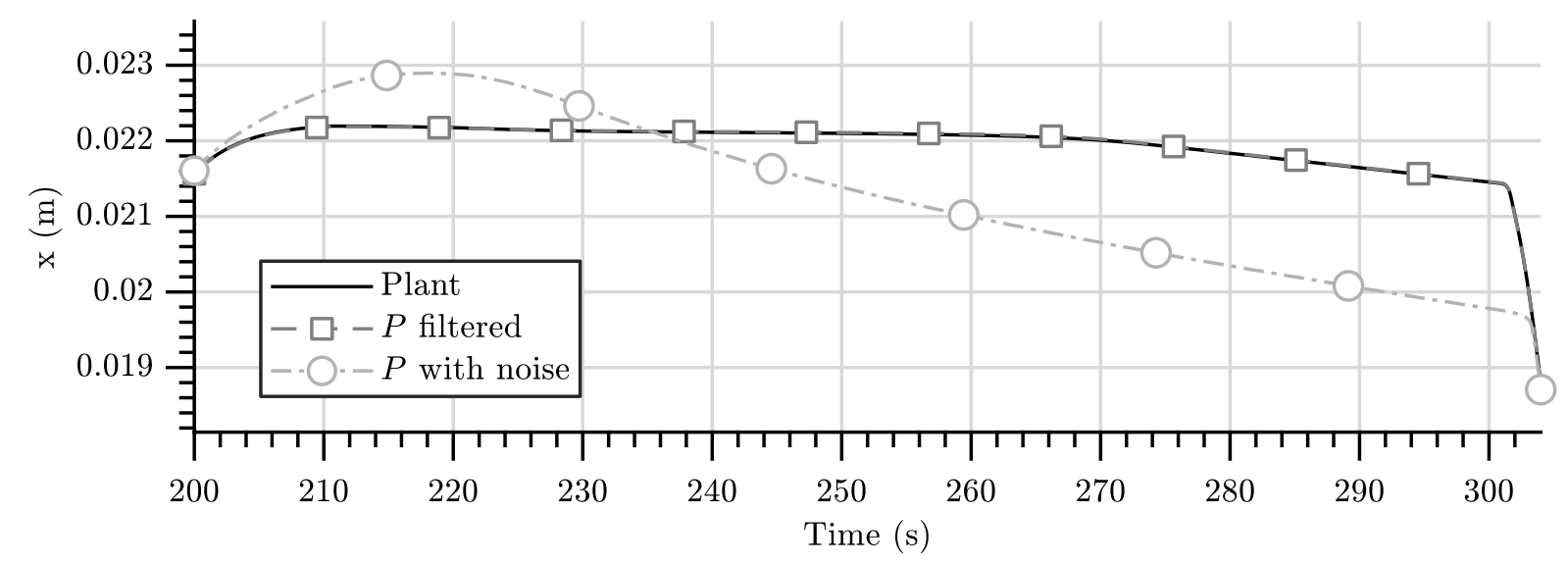

Figure 4.21 - Presliding displacement imprecision of LuGre model is negatively affected by measurement noise.

Source: from Author.

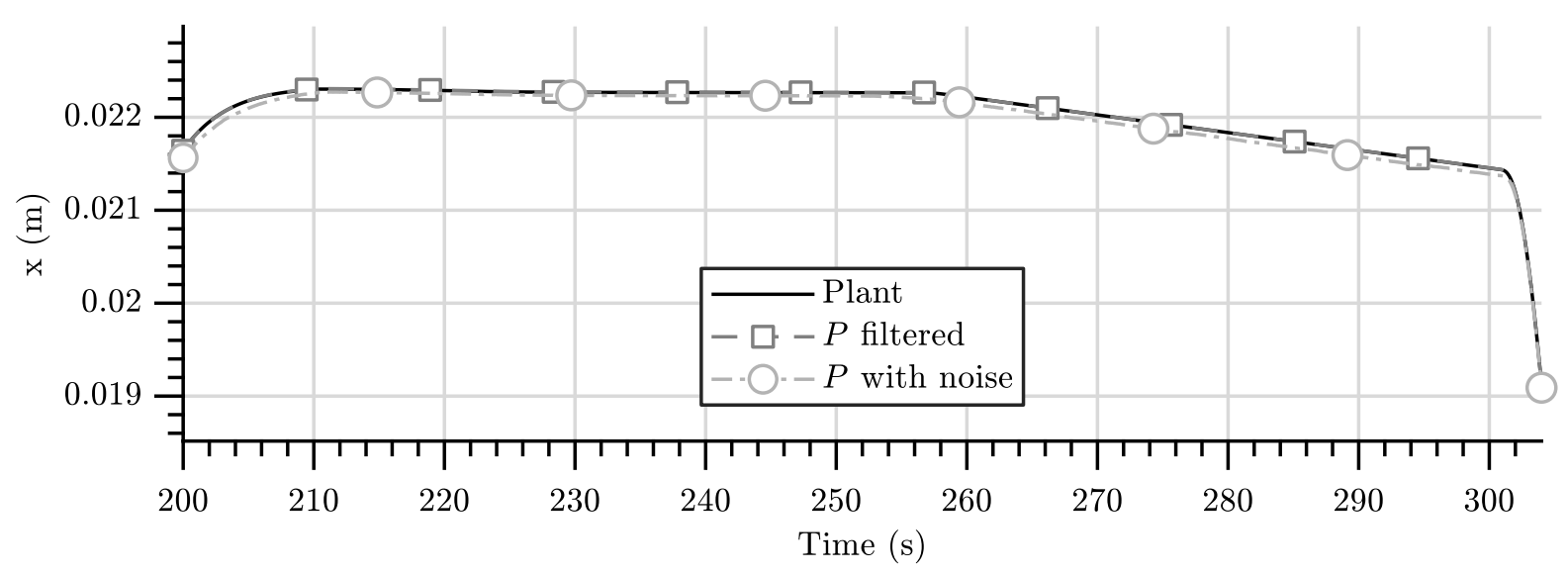

Figure 4.22 - GMS with good precision in predicting presliding displacement even in the presence of noise.

Source: from Author.

Surprisingly, the GMS model presented a slightly higher fit index than in the case without noise. Even some parameters were estimated with lower error than in the ideal case. Therefore, the GMS friction parameter estimation presents good performance, even in the presence of measurement noise. 


\subsection{Experimental Tests}

The experimental tests are performed using the valves presented in Chapter 2, applying the algorithms and guidelines suggested in Sections 4.1 and 4.2.

The valves were excited with a trapezoidal signal to estimate $S_{0}$, as shown in Figure 4.23 and the results are presented in Table 4.24 .

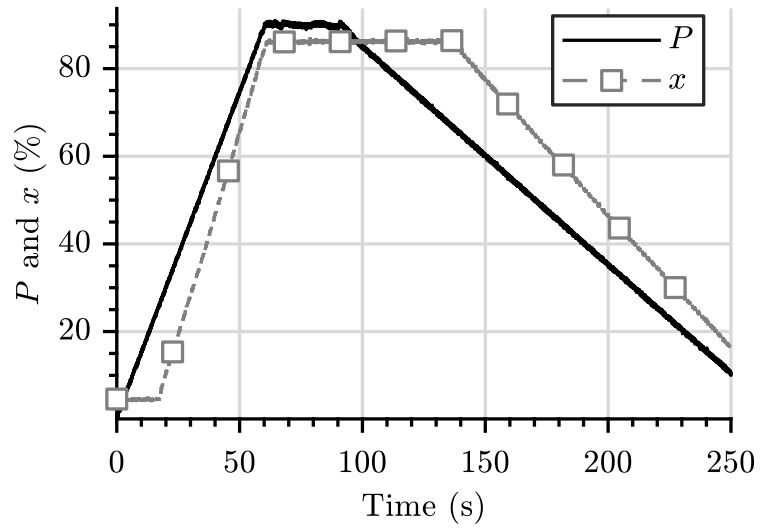

(a) Graphite gasket valve.

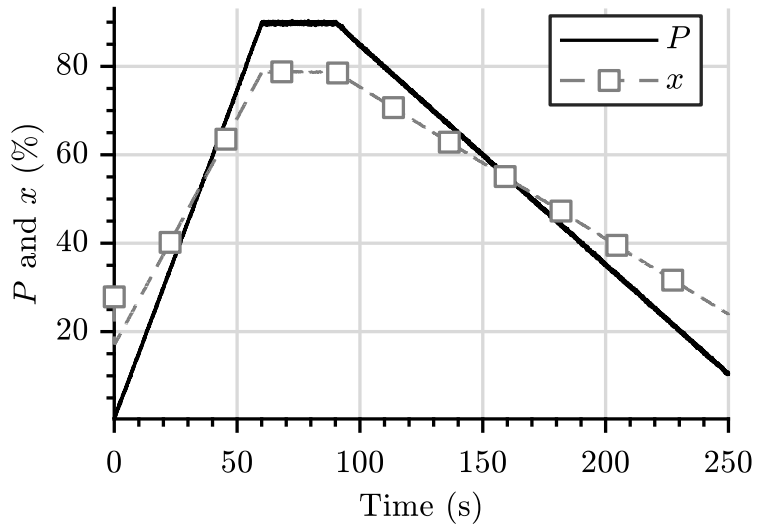

(b) Teflon gasket valve.

Figure 4.23 - Valve deadband estimation in open loop.

Source: from Author.

The I/P parameter estimation results are presented in Figures 4.24 and 4.25, and in Table 4.24. It is possible to verify that the $\mathrm{I} / \mathrm{P}$ converter time constant is very different depending on the excitation signal used, as already discussed.

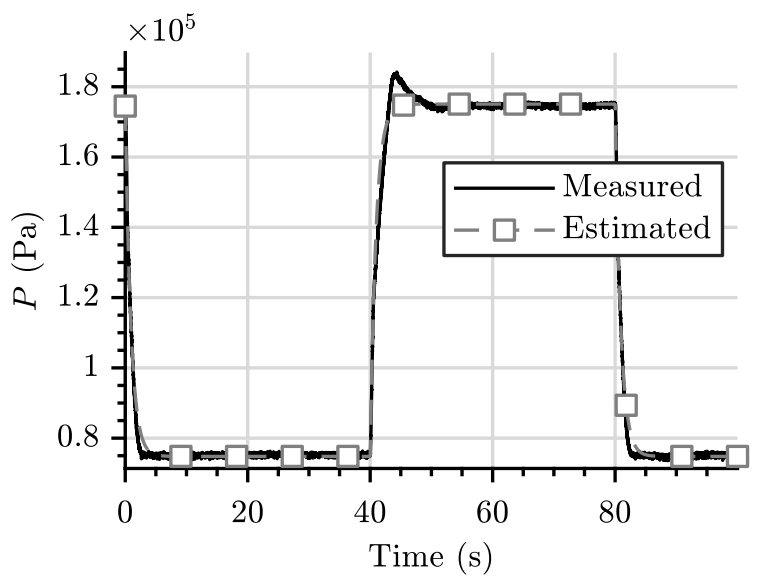

(a) Graphite gasket valve.

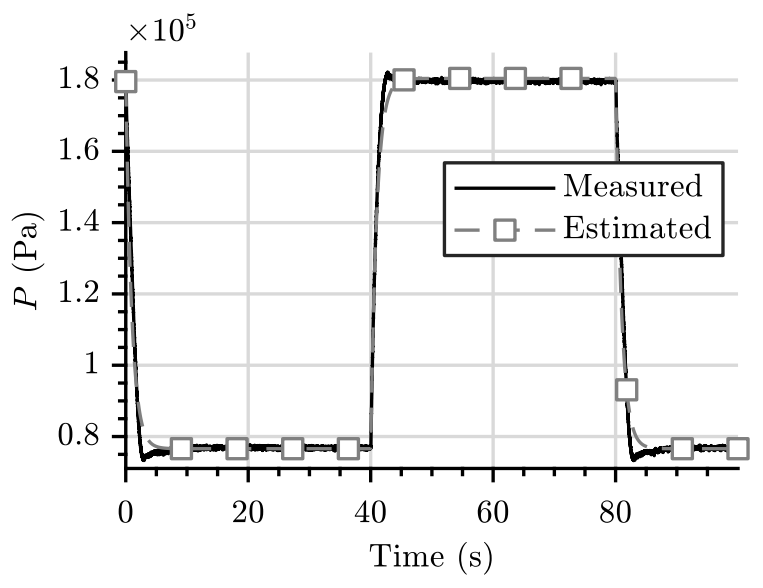

(b) Teflon gasket valve.

Figure 4.24 - Measured and estimated diaphragm pressure, using rectangular excitation.

Source: from Author. 


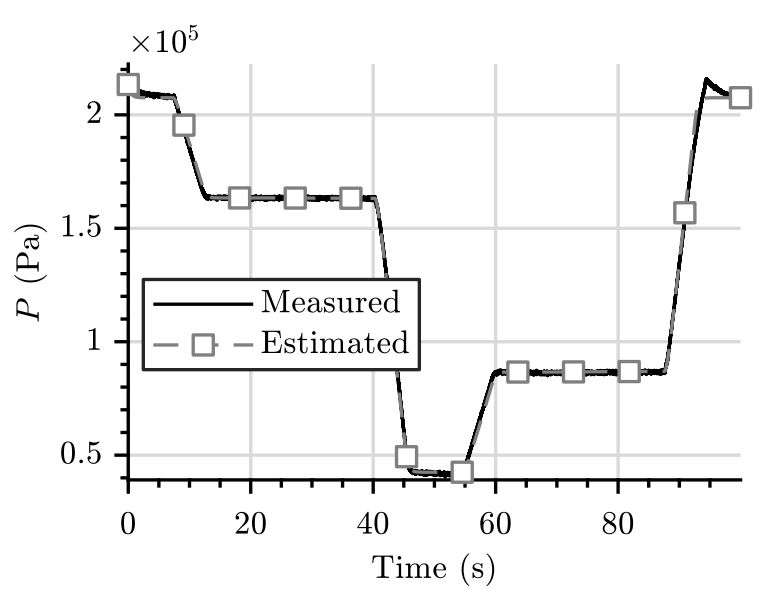

(a) Graphite gasket valve.

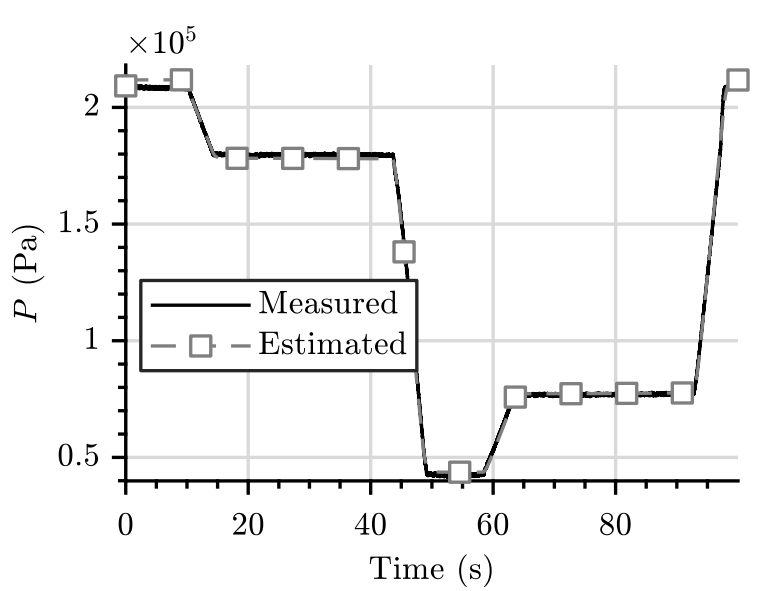

(b) Teflon gasket valve.

Figure 4.25 - Measured and estimated diaphragm pressure, using velocity excitation.

Source: from Author.

Table 4.24 - Open loop valve parameter estimation with experimental data.

\begin{tabular}{lll} 
Parameter & Graphite & Teflon \\
\hline$S_{0}(\%)$ & 23.72 & 0.52 \\
$\hat{P}_{\min }(\mathrm{Pa})$ & 41276.40 & 41861.85 \\
$\hat{K}_{P_{g}}(\mathrm{~Pa} / \%)$ & 1666.49 & 1726.54 \\
$\hat{\tau}_{\mathrm{I} / \mathrm{P}_{s}}(\mathrm{~s})$ & 0.933 & 0.979 \\
$\hat{\tau}_{\mathrm{I} / \mathrm{P}_{r}}(\mathrm{~s})$ & 0.425 & 0.438 \\
$\hat{k}(\mathrm{~N} / \mathrm{m})$ & 204670.89 & 383911.56 \\
$\operatorname{std}(\hat{k})(\mathrm{N} / \mathrm{m})$ & 8682.26 & 193.62 \\
$\hat{F}_{\text {init }}(\mathrm{N})$ & 2619.43 & -1.58 \\
\hline
\end{tabular}

To estimate $k$ and $F_{\text {init }}, v_{\mathrm{OP} \min _{R}}$ and $v_{\mathrm{OP} \max _{R}}$ were calculated using Eqs. (4.13) and (4.14) and $\hat{\tau}_{\mathrm{I} / \mathrm{P}_{s}}$, resulting in the experimental data presented in Figures 4.26 to 4.29. Note that only a few points were considered valid for the $F_{\text {init }}$ estimation, due to non stable stem velocities, but as the interest is estimating the initial force, the number of points is sufficient for a proper estimation. 


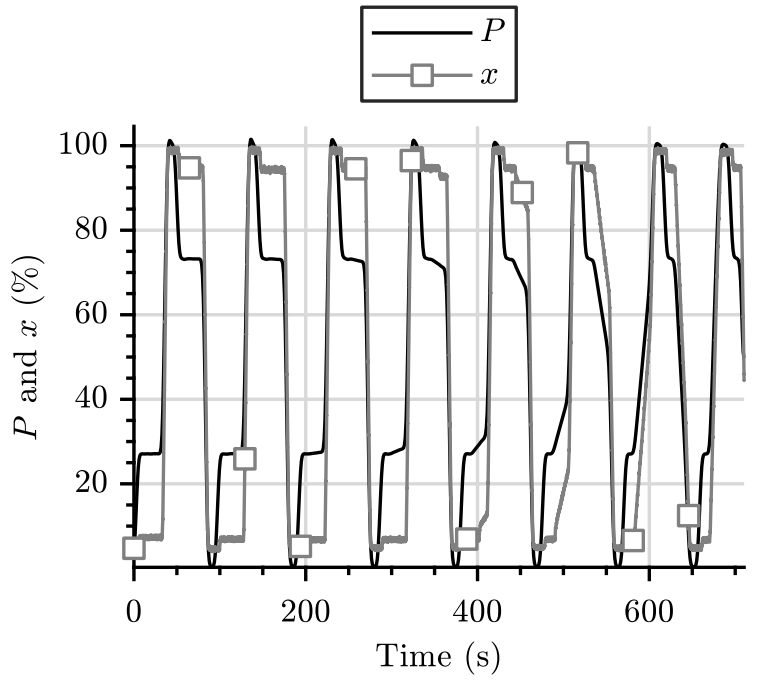

(a) Graphite gasket valve.

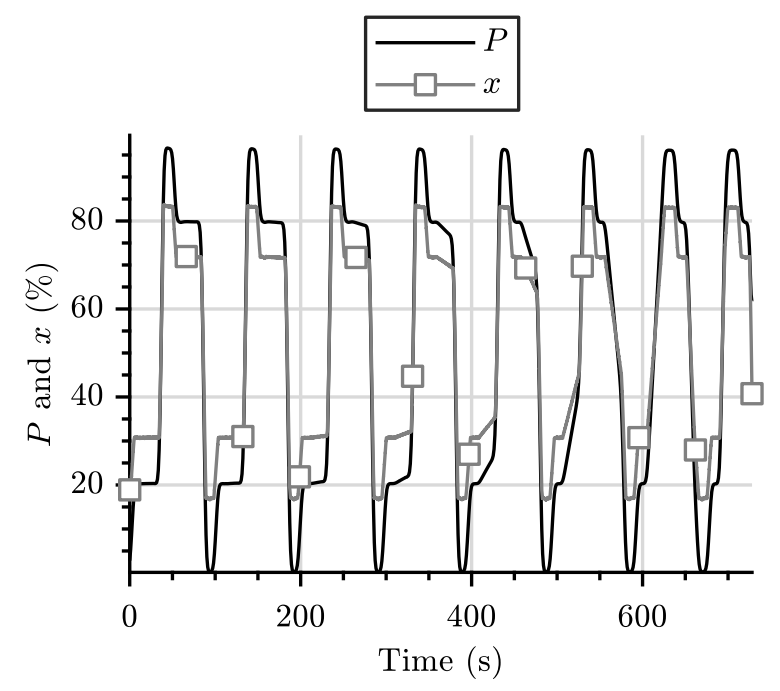

(b) Teflon gasket valve.

Figure 4.26 - Normalized diaphragm pressure and stem position for experimental open loop $k$ and $F_{\text {init }}$ estimation.

Source: from Author.

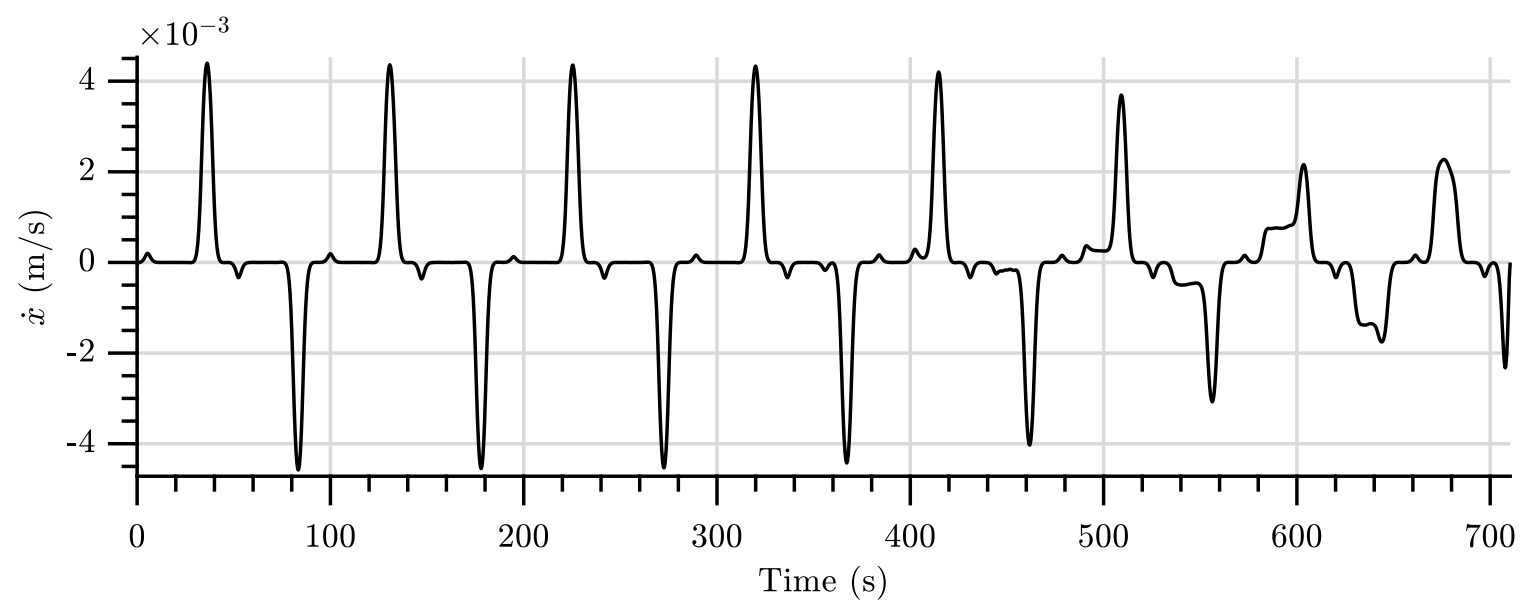

Figure 4.27 - Graphite gasket stem velocity during experiment for estimating $k$ and $F_{\text {init }}$ in open loop.

Source: from Author. 


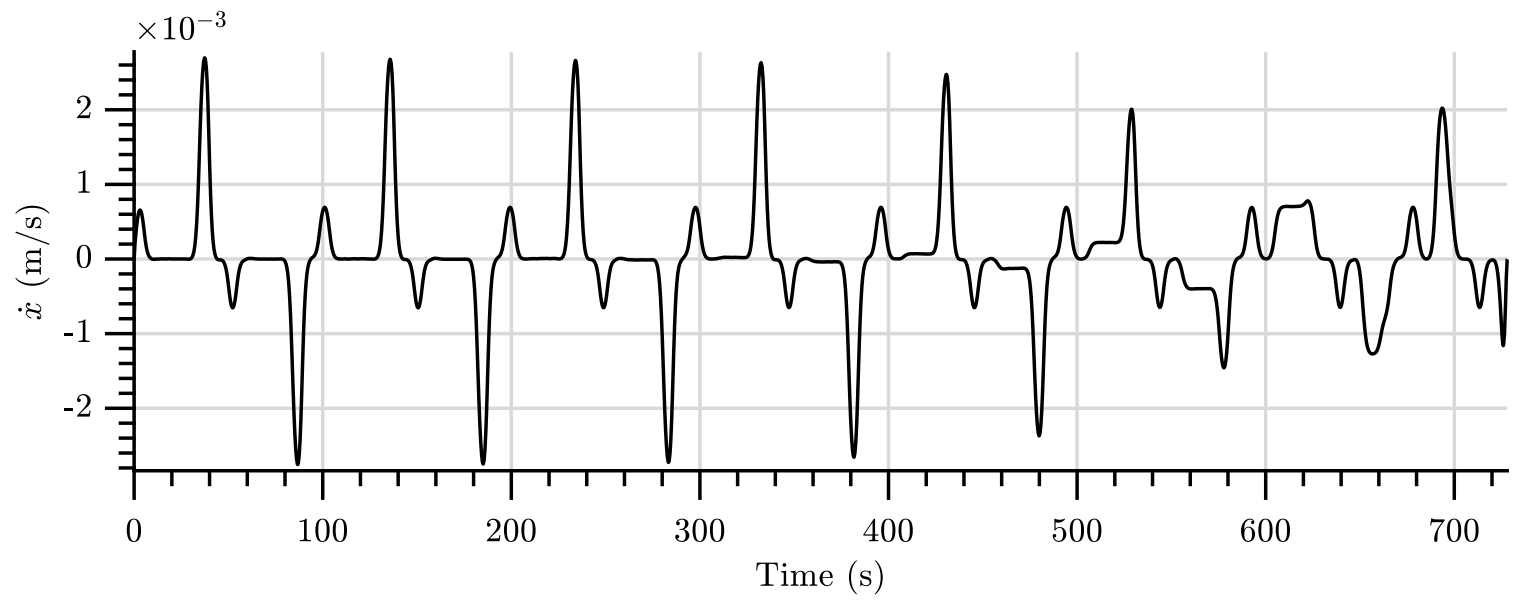

Figure 4.28 - Teflon gasket stem velocity during experiment for estimating $k$ and $F_{\text {init }}$ in open loop.

Source: from Author.

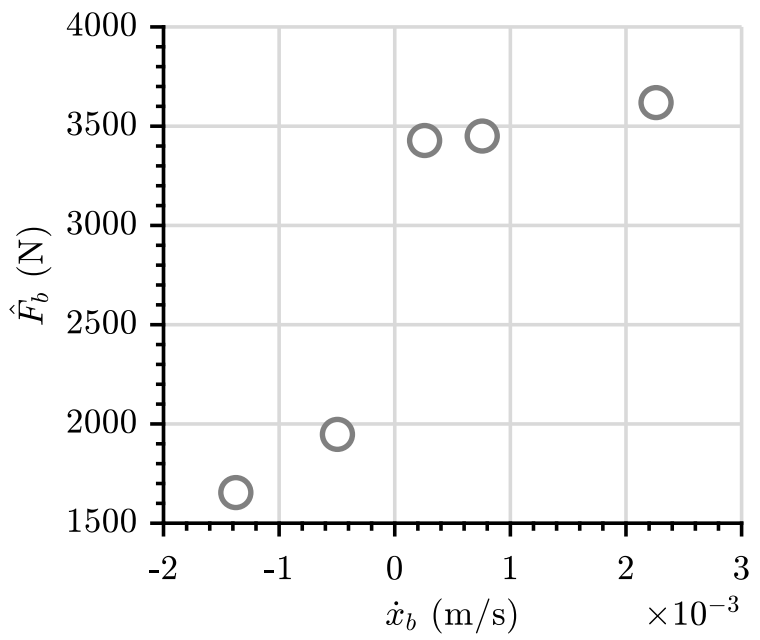

(a) Graphite gasket valve.

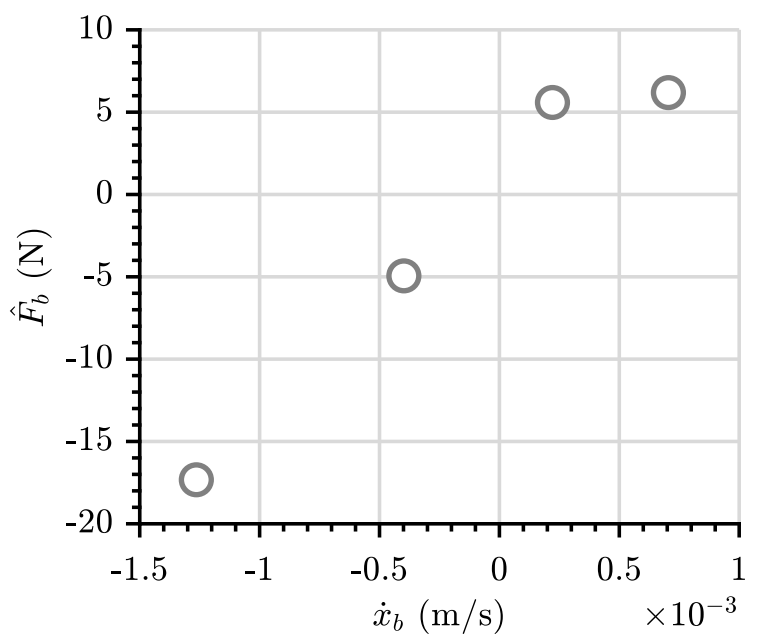

(b) Teflon gasket valve.

Figure 4.29 - Stribeck curve obtained for open loop $F_{\text {init }}$ estimation, used to solve Eq. (4.11).

Source: from Author.

The friction parameters were estimated accordingly to the simulated open loop tests presented in Section 4.3. However, as graphite gasket valve spring constant estimation presented a high standard deviation, $k$ and $F_{\text {init }}$ were estimated concomitantly with the friction parameters, with the search space calculated using Eq. (4.16). The excitation signal was designed using the guidelines of Section 4.2 , with $T_{s_{\max }}=40 \mathrm{~s}$ and $v_{s_{\min }}=10^{-6} \mathrm{~m} / \mathrm{s}$, $S_{0}$ as in Table 4.24 and $T_{s_{\min }}$ and $v_{s_{\max }}$ calculated as in Eqs. (4.19) and (4.20). The signals used for the friction parameters estimation are presented in Figures 4.30 and 4.31. 


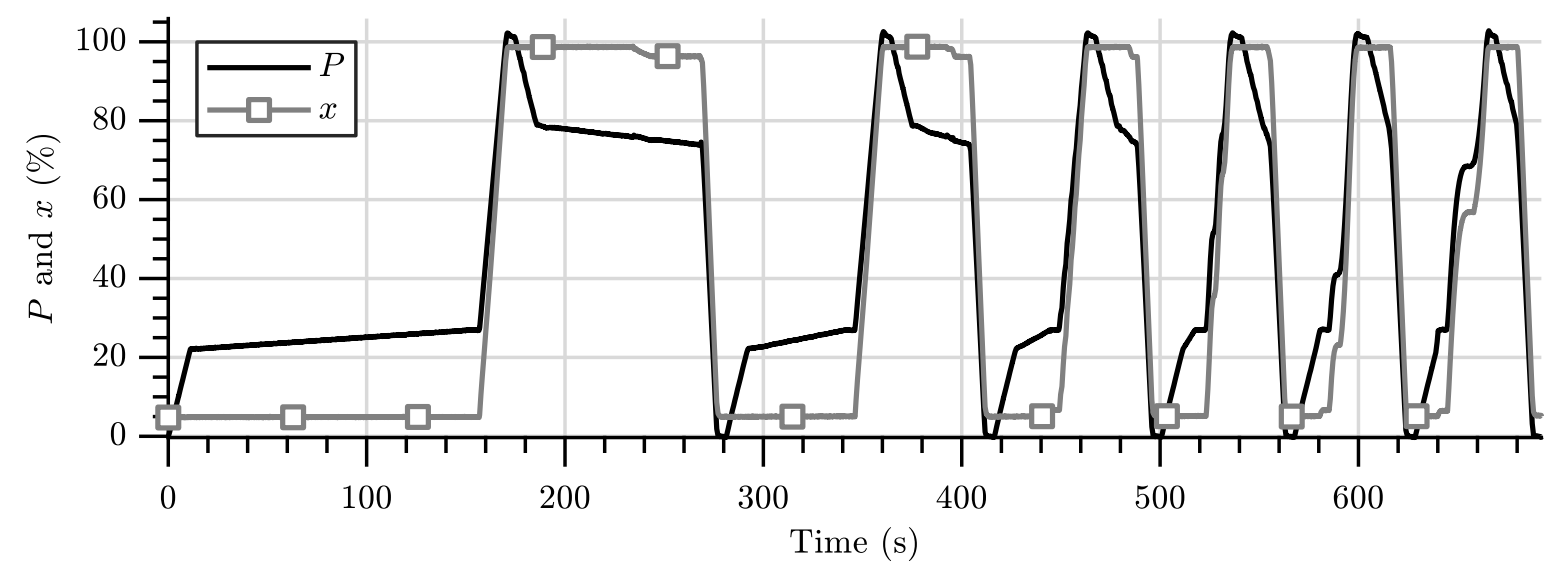

Figure 4.30 - Filtered signals used for estimating the graphite gasket valve friction parameters, $k$ and $F_{\text {init }}$.

Source: from Author.

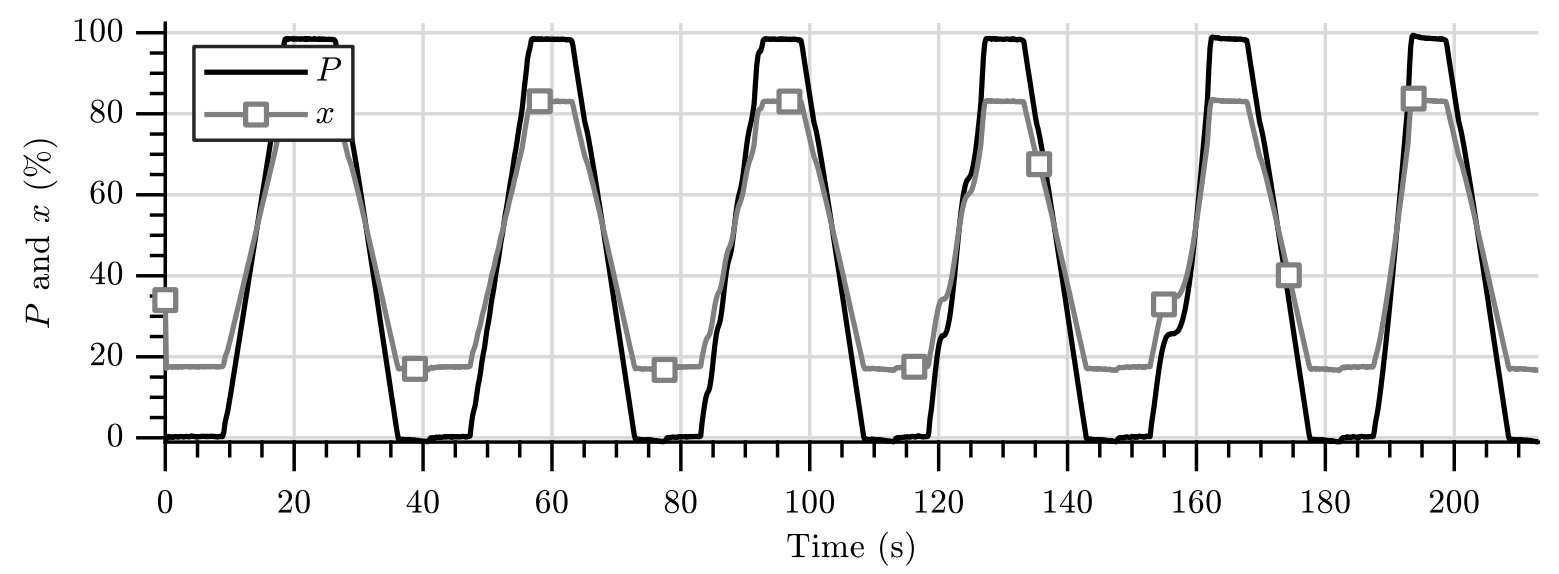

Figure 4.31 - Filtered signals used for estimating the teflon gasket valve friction parameters.

Source: from Author.

To validate the models with a different dataset, the fit index is also evaluated using a ramp signal with aleatory velocity as excitation signal. The estimation results are presented in Tables 4.25 to 4.30 . With respect to the graphite gasket valve, it is possible to verify that the Kano model has the lowest performance. The Karnopp, LuGre and GMS efficiencies are very good and have similar $f i t$ indexes. It is interesting to note that the $\hat{F}_{s}$ values are close to each other, as expected. As for the teflon gasket valve, once more the Kano model presented the worst performance, whereas the other models show outstanding prediction abilities either for the estimation or validation datasets. 
Table 4.25 - Open loop graphite gasket valve model estimation performance.

\begin{tabular}{lrrrr} 
Model & fit $(\%)$ & fit val. (\%) & Run Time (h) & Func. Evals. \\
\hline Kano & 97.76 & 94.82 & 0.01 & 2515 \\
Karnopp & 99.11 & 96.83 & 26.20 & 37019 \\
LuGre & 99.11 & 96.83 & 37.57 & 52253 \\
GMS & 99.08 & 96.74 & 76.10 & 60744 \\
\hline
\end{tabular}

fit val.: indicates to the fit index evaluated using the validation dataset and Func. Evals.: refers to the number of objective function evaluations.

Source: from Author.

Table 4.26 - Open loop teflon gasket valve model estimation performance.

\begin{tabular}{lrrrr} 
Model & fit (\%) & fit val. (\%) & Run Time (h) & Func. Evals. \\
\hline Kano & 97.93 & 97.78 & 0.01 & 2368 \\
Karnopp & 99.13 & 99.35 & 1.03 & 4914 \\
LuGre & 99.15 & 99.37 & 3.30 & 13398 \\
GMS & 99.16 & 99.40 & 26.94 & 80012
\end{tabular}

fit val.: indicates to the fit index evaluated using the validation dataset and Func. Evals.: refers to the number of objective function evaluations.

Source: from Author.

Table 4.27 - Open loop Kano estimated parameters.

\begin{tabular}{lccc} 
& $\hat{S}(\%)$ & $\hat{J}(\%)$ & $\hat{D}(\%)$ \\
\hline Graphite & 27.13 & 0.75 & 14.36 \\
Teflon & 0.86 & 0.00 & 0.00 \\
\hline \multicolumn{4}{c}{ Source: from Author. }
\end{tabular}

Table 4.28 - Open loop Karnopp estimated parameters.

\begin{tabular}{lcccccc} 
& $\hat{k}(\mathrm{~N} / \mathrm{m})$ & $\hat{F}_{\text {init }}(\mathrm{N})$ & $\hat{F}_{c}(\mathrm{~N})$ & $\hat{F}_{s}(\mathrm{~N})$ & $\hat{F}_{v}(\mathrm{Ns} / \mathrm{m})$ & $\hat{v}_{s}(\mathrm{~m} / \mathrm{s})$ \\
\hline Graphite & 203527.8 & 2577.6 & 717.9 & 938.3 & 54836.8 & $4.23 \cdot 10^{-3}$ \\
Teflon & - & - & 0 & 0 & 5780.47 & $3.87 \cdot 10^{-2}$ \\
\hline \multicolumn{6}{c}{ Source: from Author. }
\end{tabular}

Table 4.29 - Open loop LuGre estimated parameters.

\begin{tabular}{lcccccc} 
& $\hat{k}(\mathrm{~N} / \mathrm{m})$ & $\hat{F}_{\text {init }}(\mathrm{N})$ & $\hat{F}_{c}(\mathrm{~N})$ & $\hat{F}_{s}(\mathrm{~N})$ & $\hat{F}_{v}(\mathrm{Ns} / \mathrm{m})$ & $\hat{v}_{s}(\mathrm{~m} / \mathrm{s})$ \\
\hline Graphite & 203495.8 & 2578.3 & 623.7 & 939.5 & 46624.4 & $6.42 \cdot 10^{-3}$ \\
Teflon & - & - & 1.65 & 2.05 & 3534.69 & $1.41 \cdot 10^{-5}$ \\
\hline
\end{tabular}

\begin{tabular}{lcc} 
Model & $\hat{\sigma}_{0}(\mathrm{~N} / \mathrm{m})$ & $\hat{\sigma}_{1}(\mathrm{Ns} / \mathrm{m})$ \\
\hline Graphite & $1.07 \cdot 10^{9}$ & 110182.2 \\
Teflon & $3.61 \cdot 10^{5}$ & 527.46 \\
\hline \multicolumn{3}{c}{ Source: from Author. }
\end{tabular}


Table 4.30 - Open loop GMS estimated parameters.

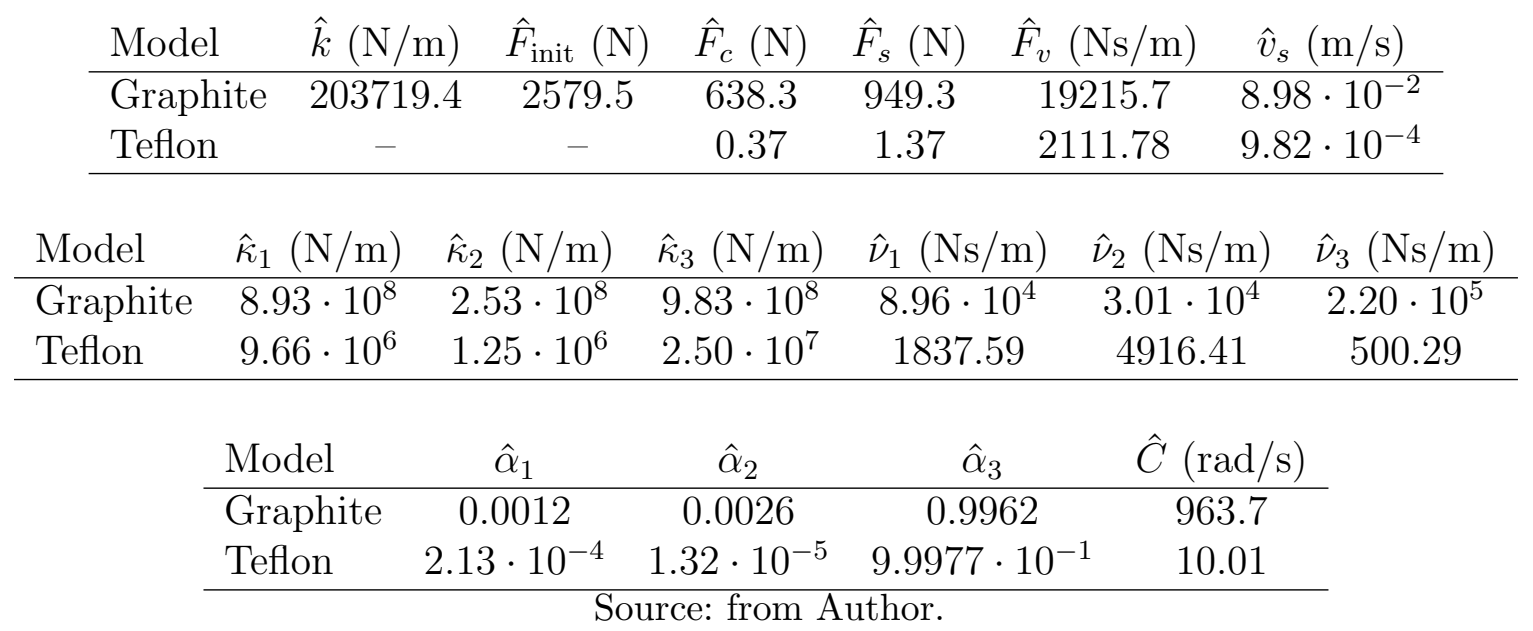

\subsubsection{Graphite Gasket Model Prediction Analysis}

For a qualitative analysis of the prediction efficiency of the graphite gasket valve models, the results are presented in Figures 4.32 to 4.43. It is possible to note that the Kano model presented the higher residual values. The other model residuals are very similar with a few visible differences. As the system velocity is important for friction compensation, as it evidence the stick-slip phenomena that is able to induce limit cycles, the comparison between the measured and estimated stem velocity is presented, as well. The Kano velocity prediction is poor, as it presented stick-slip motion that is not visible in the experimental data. Once more, the other models showed quite good stem velocity prediction precisions, with the only visible difference being a higher noise at low velocity prediction, regarding the GMS model. 

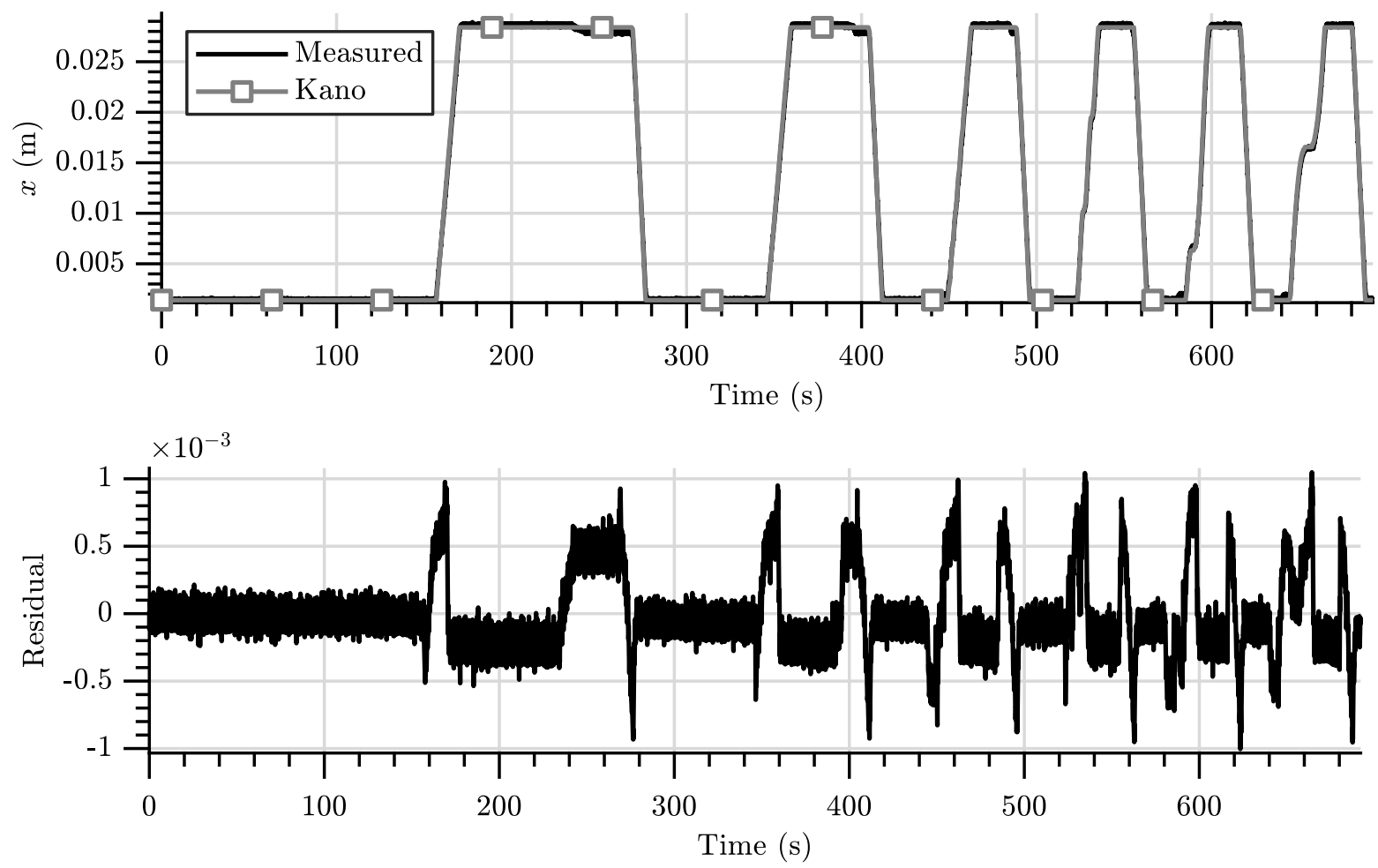

Figure 4.32 - Kano model graphite gasket valve residual using the estimation dataset.

Source: from Author.
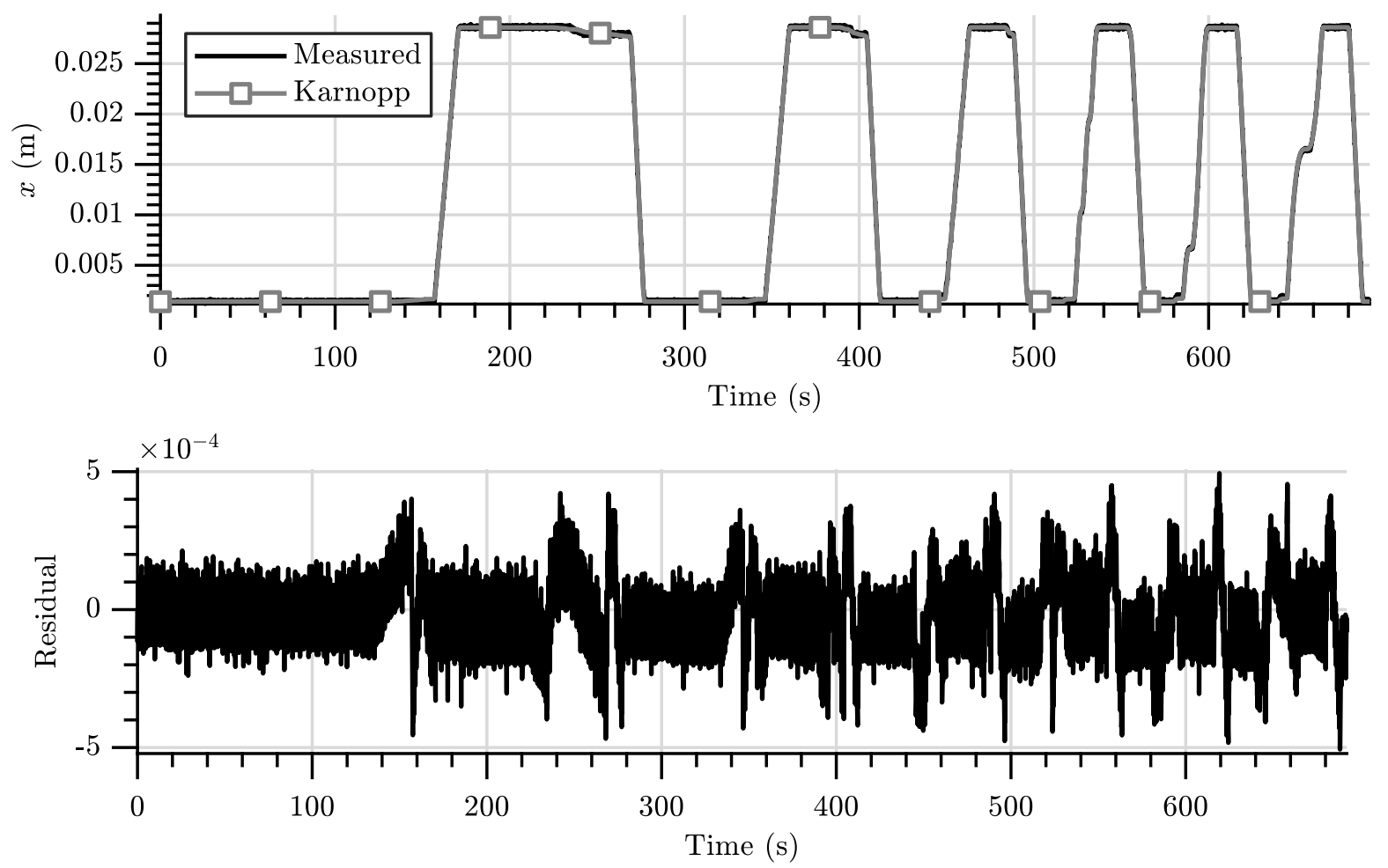

Figure 4.33 - Karnopp model graphite gasket valve residual using the estimation dataset.

Source: from Author. 

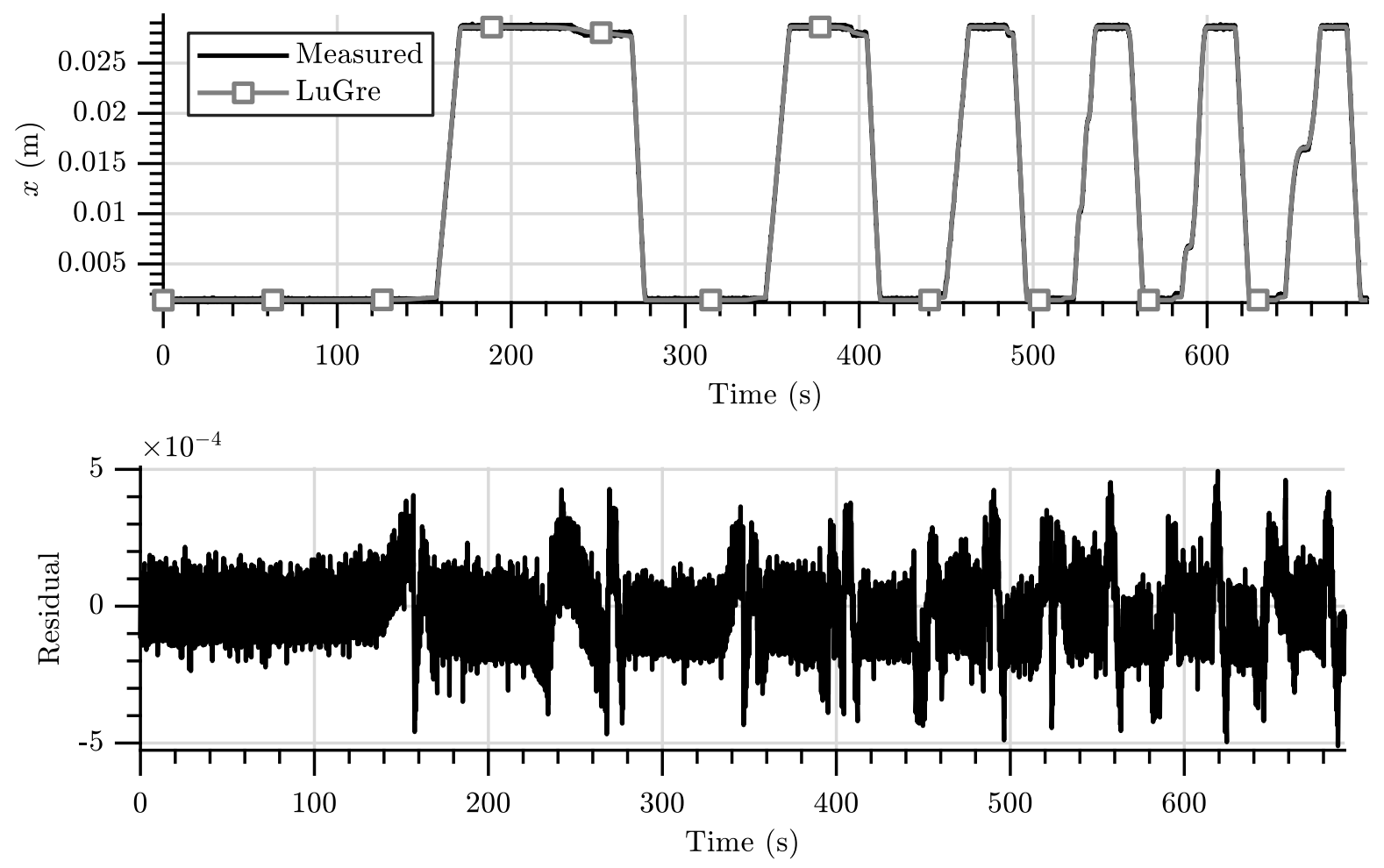

Figure 4.34 - LuGre model graphite gasket valve residual using the estimation dataset.

Source: from Author.
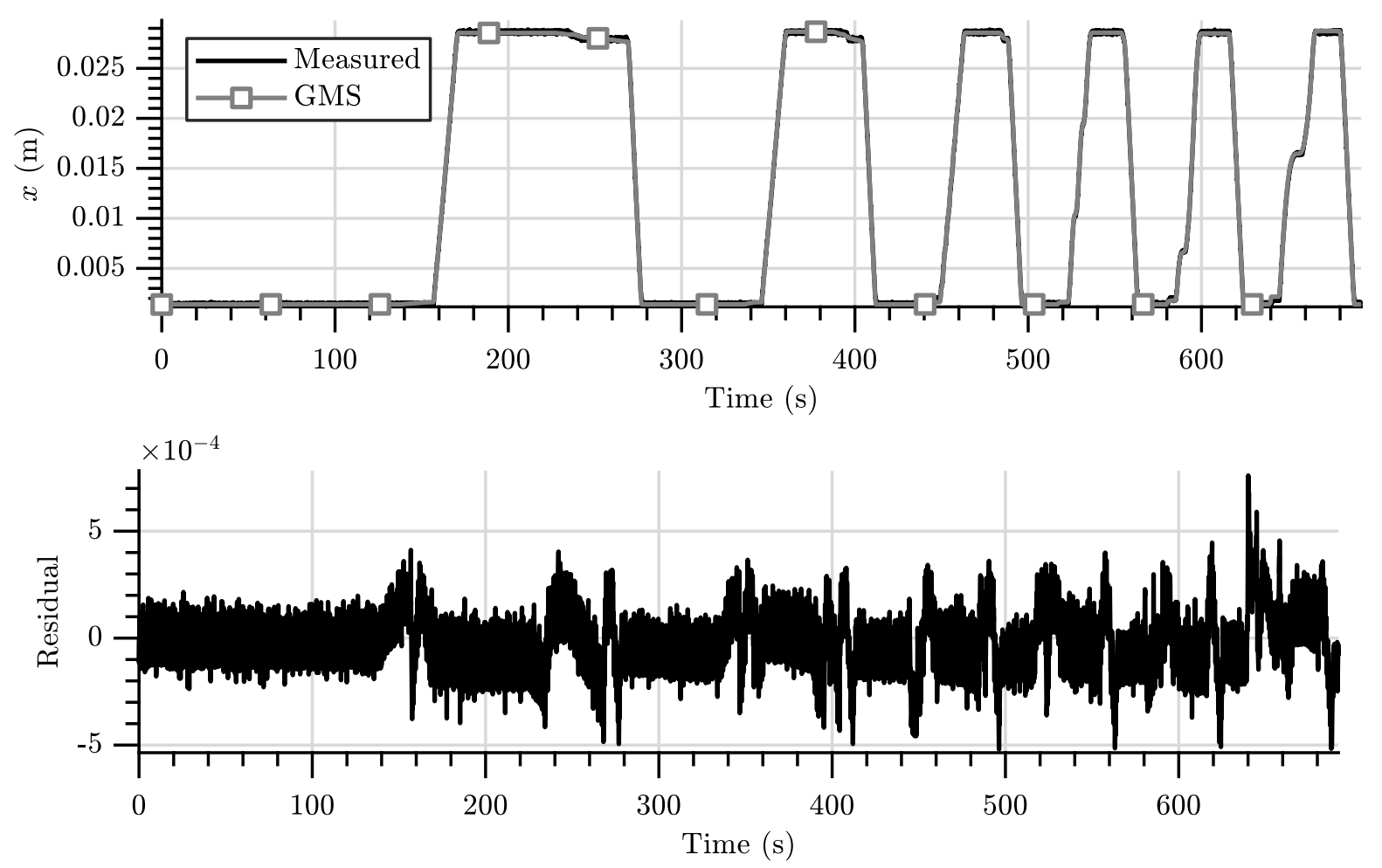

Figure 4.35 - GMS model graphite gasket valve residual using the estimation dataset.

Source: from Author. 

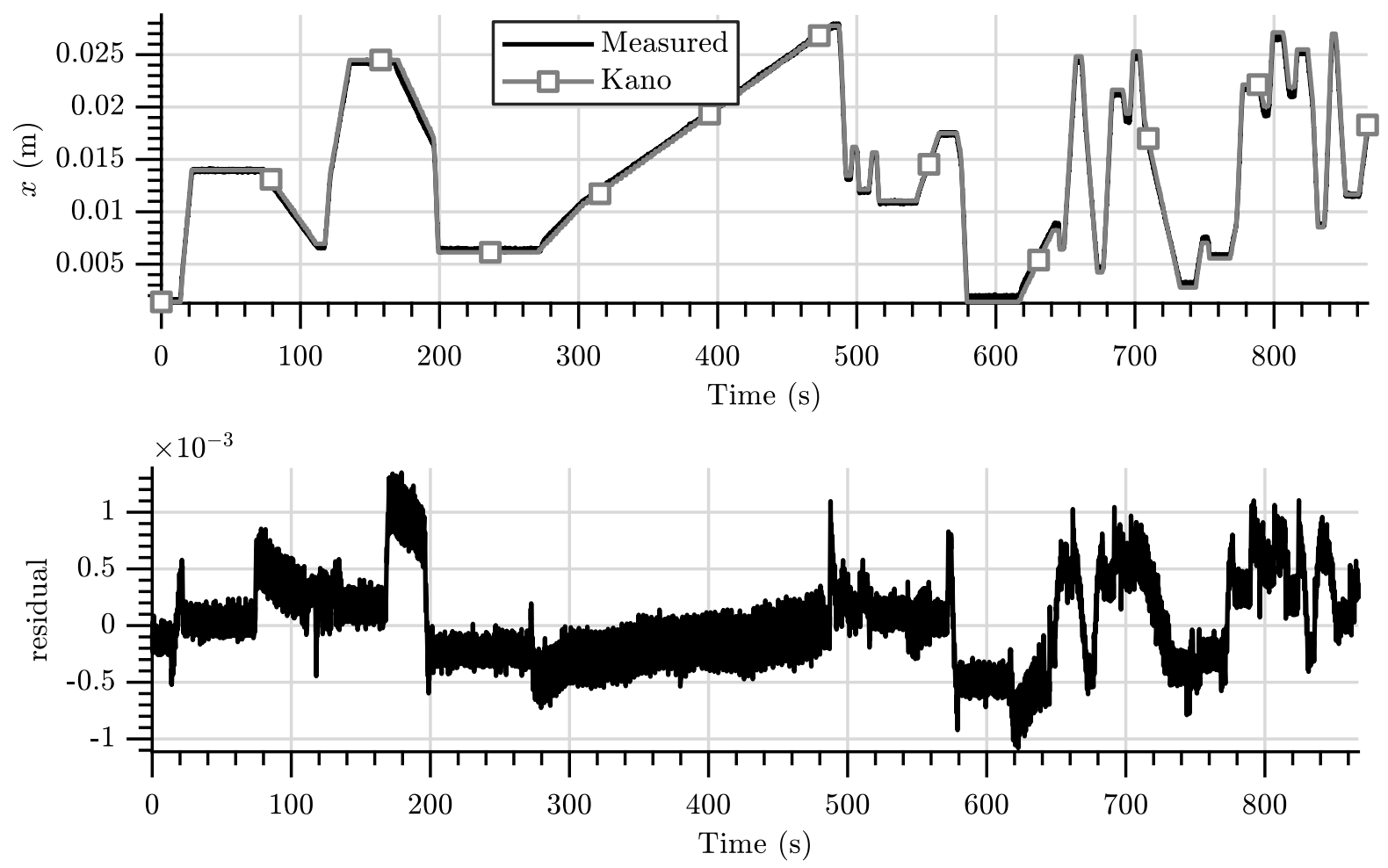

Figure 4.36 - Kano model graphite gasket valve residual using the validation dataset.

Source: from Author.
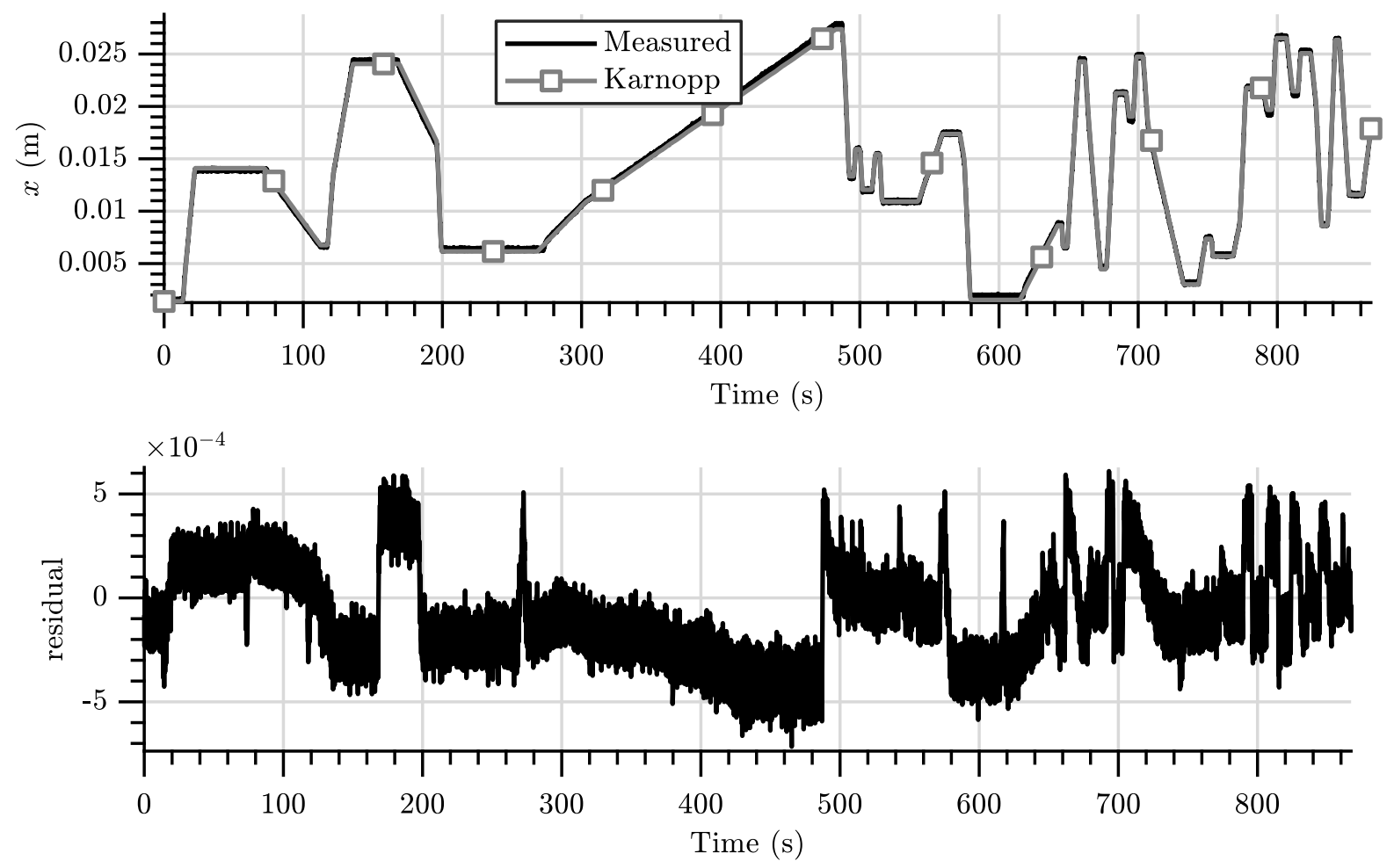

Figure 4.37 - Karnopp model graphite gasket valve residual using the validation dataset.

Source: from Author. 

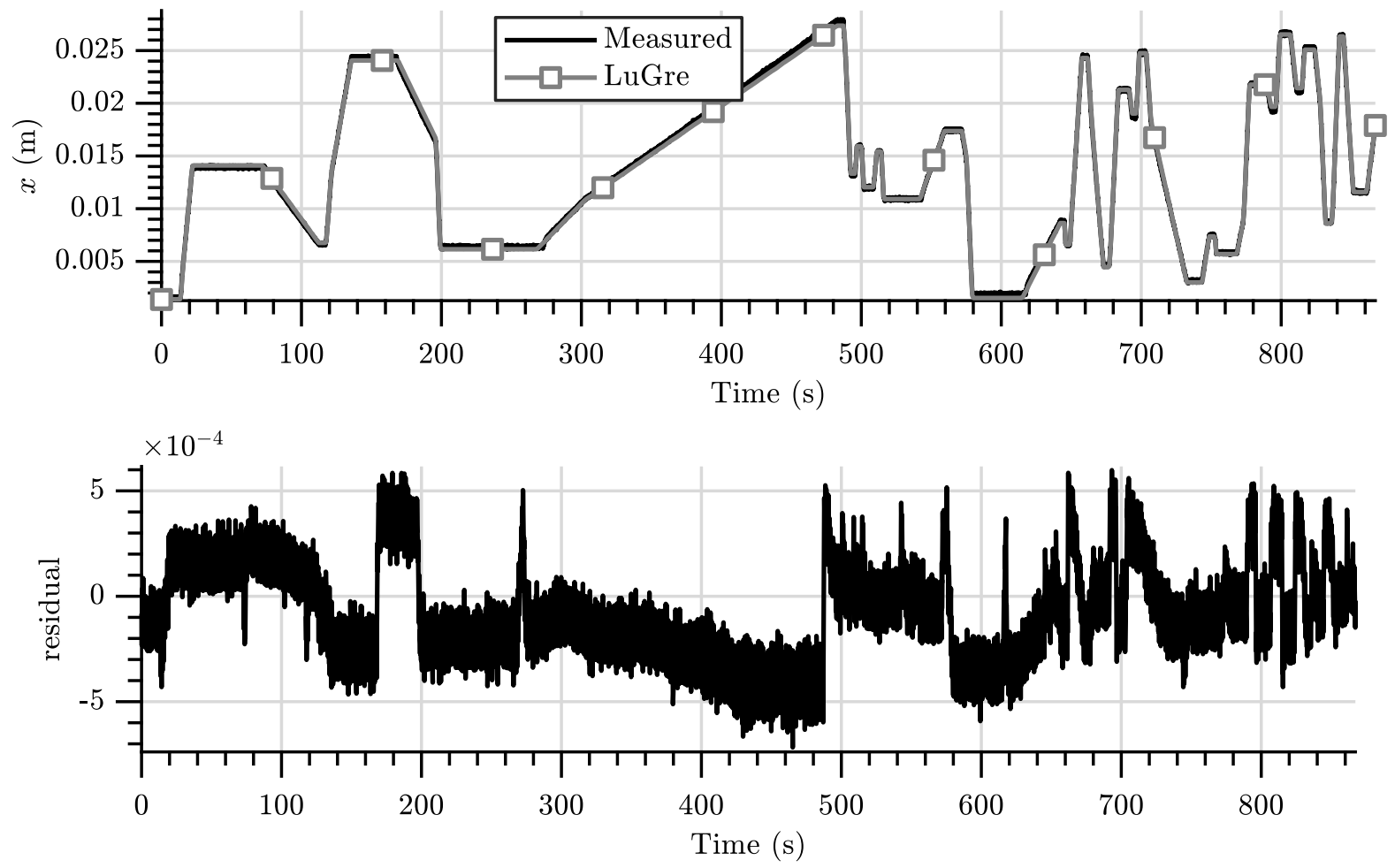

Figure 4.38 - LuGre model graphite gasket valve residual using the validation dataset.

Source: from Author.
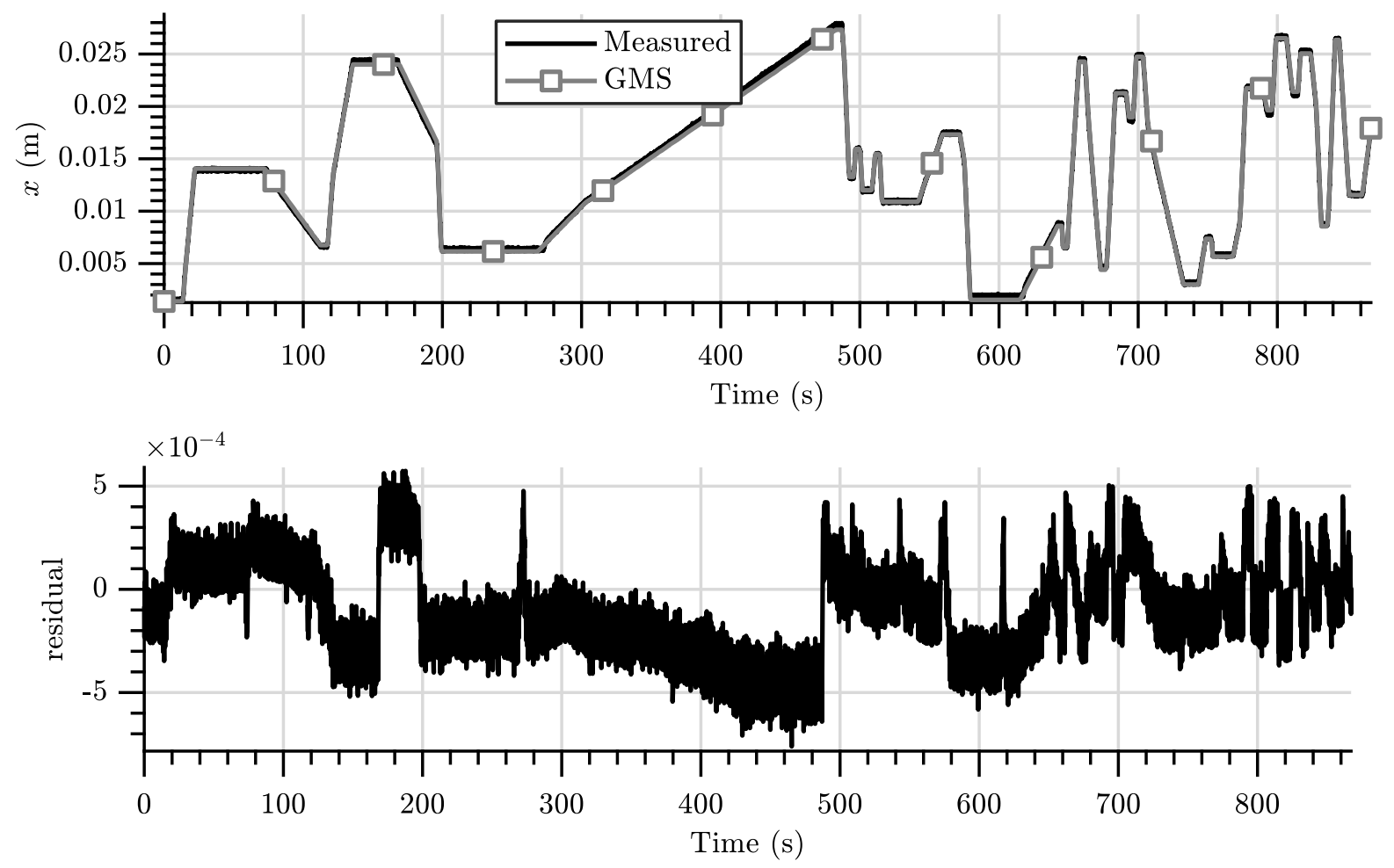

Figure 4.39 - GMS model graphite gasket valve residual using the validation dataset.

Source: from Author. 


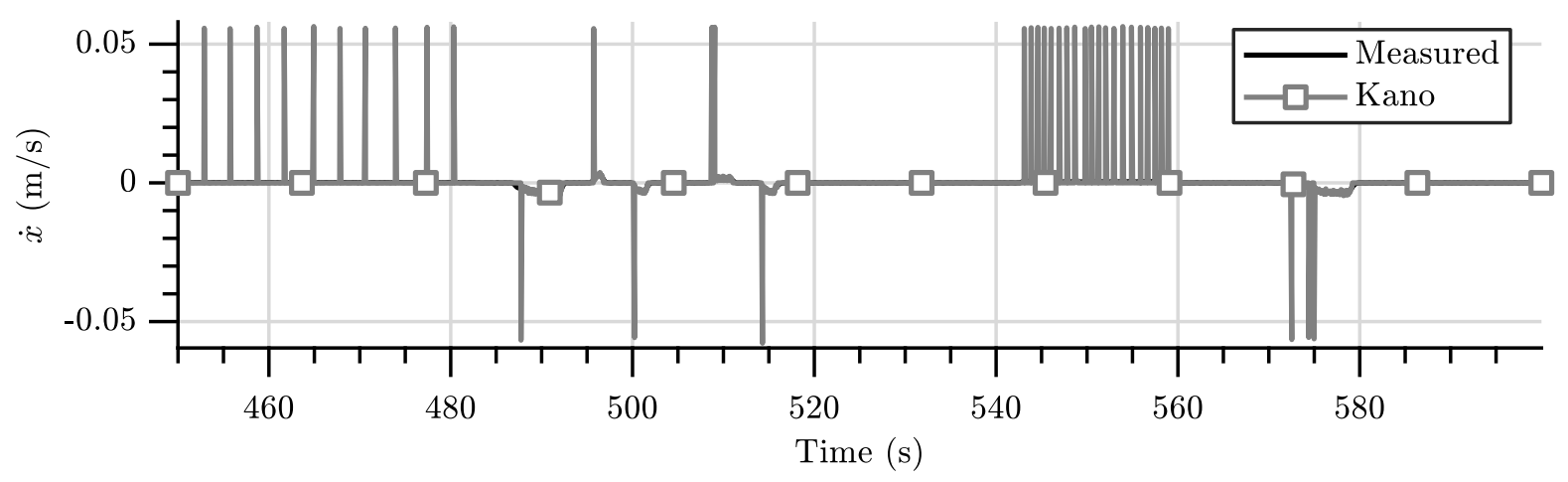

Figure 4.40 - Kano model graphite gasket valve stem velocity prediction (450 s to $600 \mathrm{~s}$ ) using the validation dataset.

Source: from Author.

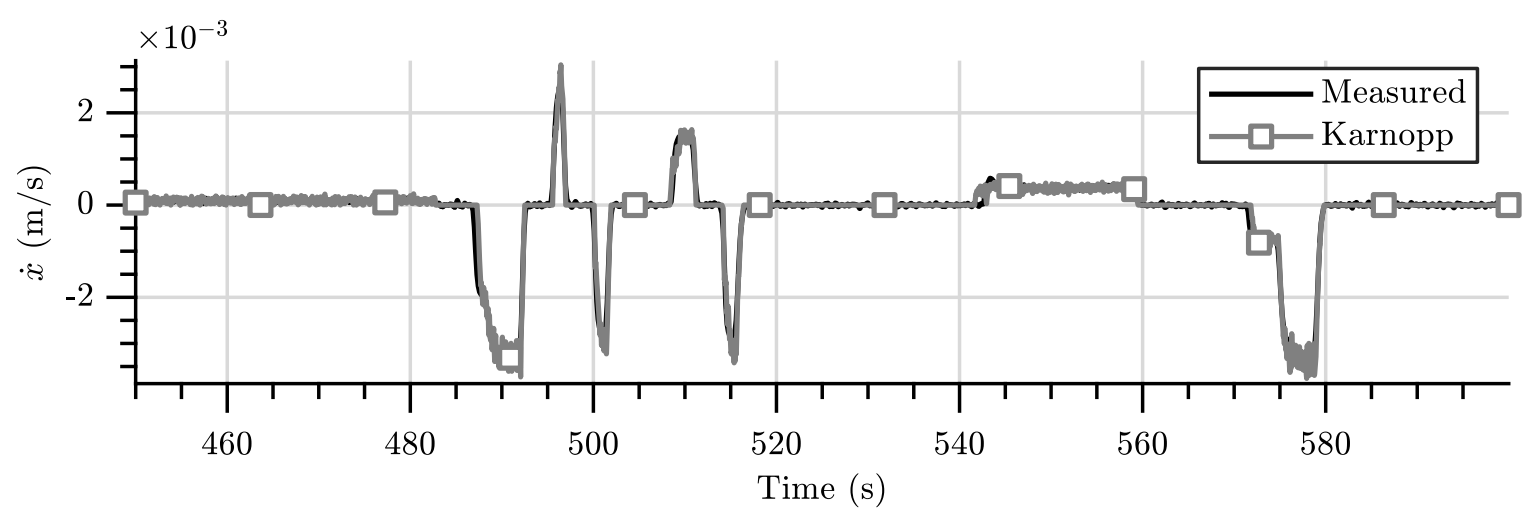

Figure 4.41 - Karnopp model graphite gasket valve stem velocity prediction (450 $\mathrm{s}$ to $600 \mathrm{~s}$ ) using the validation dataset.

Source: from Author.

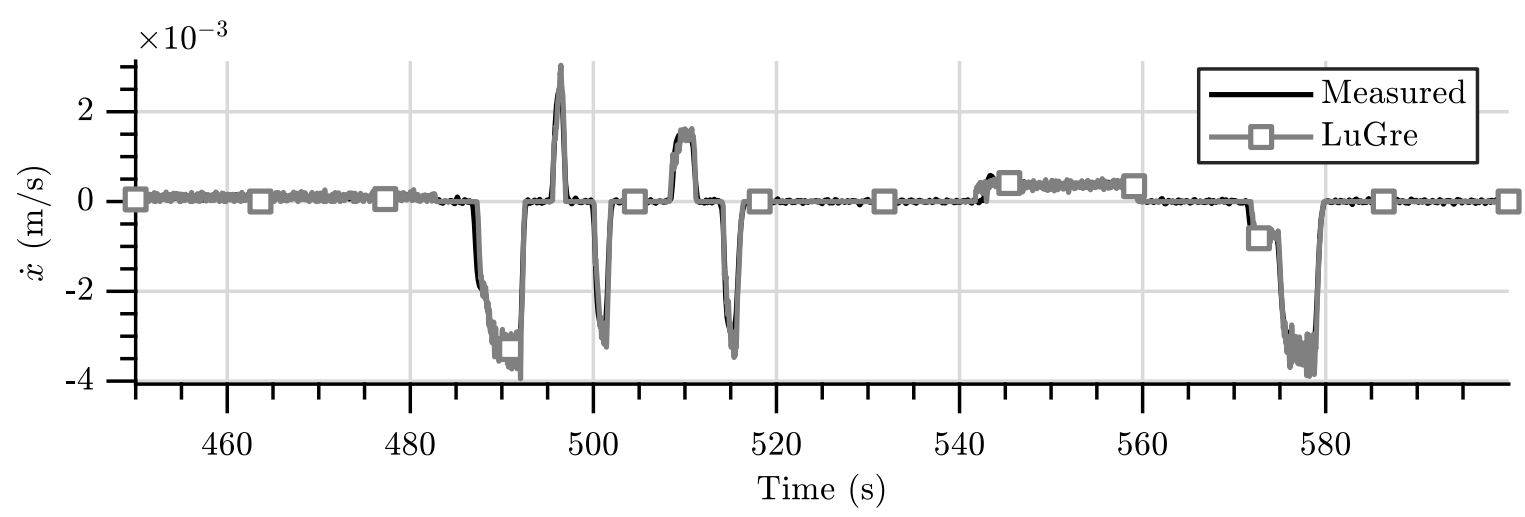

Figure 4.42 - LuGre model graphite gasket valve stem velocity prediction (450 s to $600 \mathrm{~s}$ ) using the validation dataset.

Source: from Author. 


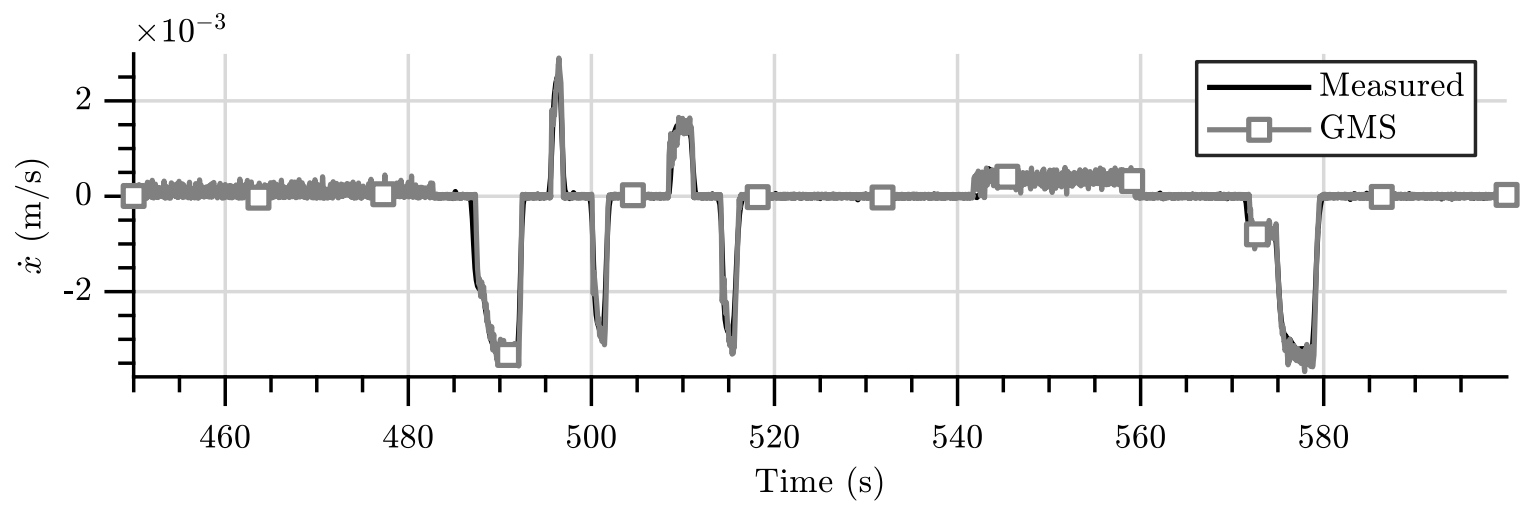

Figure 4.43 - GMS model graphite gasket valve stem velocity prediction (450 s to $600 \mathrm{~s}$ ) using the validation dataset.

Source: from Author.

As another resource for analyzing the graphite gasket valve model prediction efficiency, the residual distribution is presented in Figures 4.44 to 4.46 . From the error distribution with the estimation dataset plots, it is possible to verify that the Karnopp, LuGre and GMS model residuals presented a Gaussian like distribution, which is desirable because it indicates that the residual is approximately an aleatory variable. The distributions show a slight bias, though, which may indicate a constant error, probably due to $k$ or $F_{\text {init }}$ errors. The Kano distribution is not symmetric and presents three modes, which implies that the model is not able to predict the plant behavior perfectly.

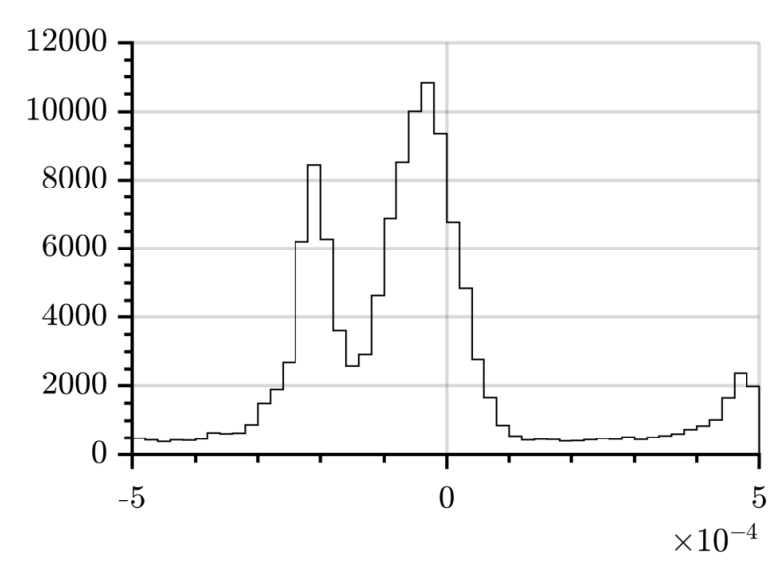

(a) Kano model.

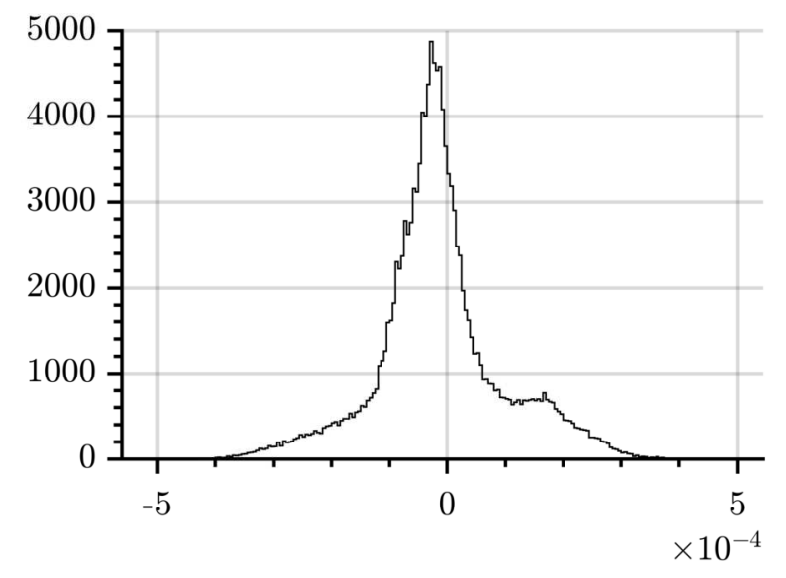

(b) Karnopp model.

Figure 4.44 - Kano and Karnopp graphite gasket valve prediction error distribution using the estimation dataset.

Source: from Author. 


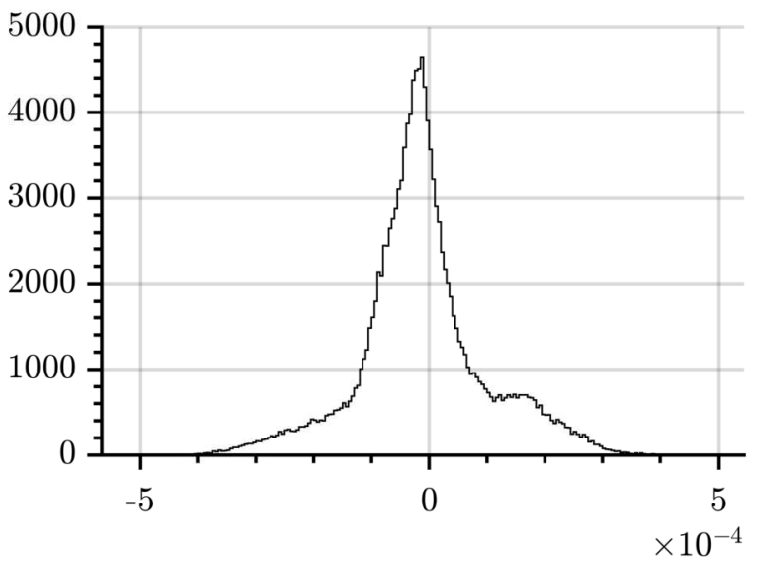

(a) LuGre model.

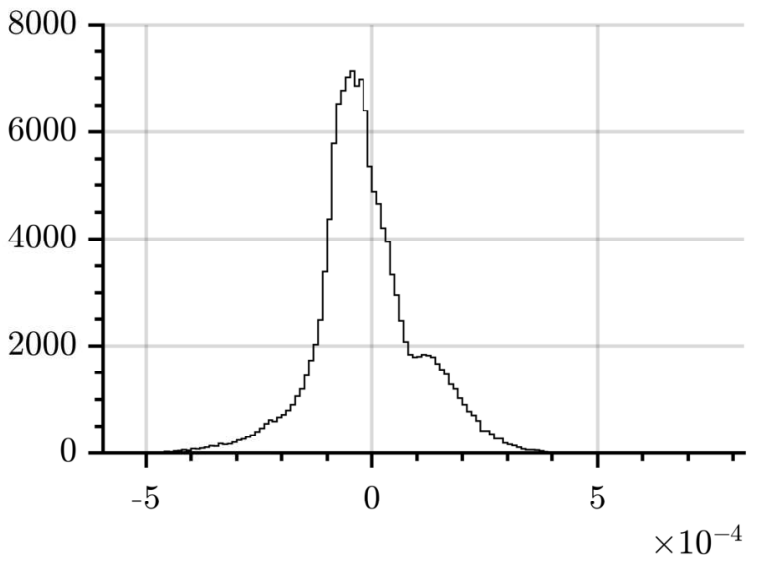

(b) GMS model.

Figure 4.45 - LuGre and GMS graphite gasket valve prediction error distribution using the estimation dataset.

Source: from Author.

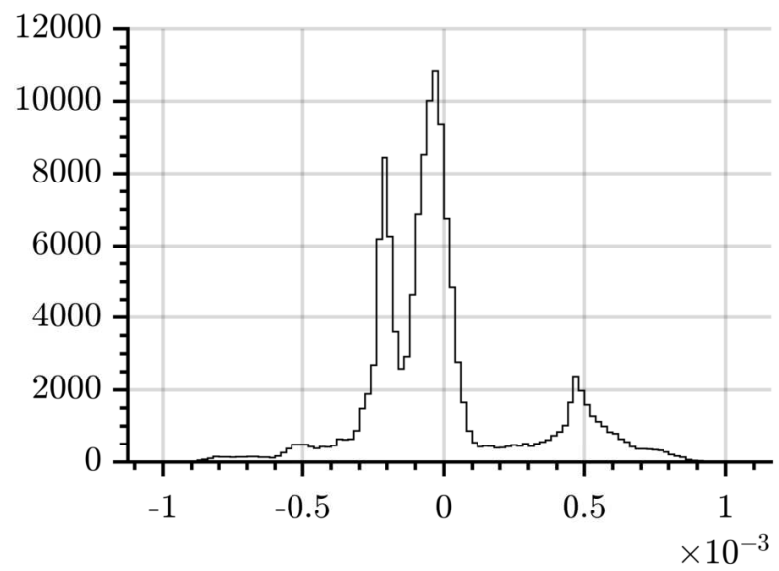

Figure 4.46 - Kano graphite gasket valve prediction error distribution using the estimation dataset, with no scaling in $\mathrm{x}$ axis.

The correlation coefficient, defined as:

$$
r(a, b)=\frac{\sum_{n=1}^{n_{f}}(a[n]-\mu(a))(b[n]-\mu(b))}{\sqrt{\sum_{n=1}^{n_{f}}(a[n]-\mu(a))^{2}} \sqrt{\sum_{n=1}^{n_{f}}(b[n]-\mu(b))^{2}}},
$$

is calculated to determine the correlation of some variable combinations, as shown in Table 4.31. Low values of $r\left(e_{x}, \hat{x}\right)$ and $r\left(e_{x}, \dot{x}\right)$ are indications of successful parameter estimation. As already observed in the visual analysis, the Kano model has the worst correlation coefficients, and $r(\hat{\dot{x}}, \dot{x})$ shows that the Kano model has a poor performance for predicting the stem velocity. The other models presented pretty good and similar correlation coefficients between the measured and simulated stem position and velocity. 
Table 4.31 - Statistical measures of the graphite gasket valve prediction.

\begin{tabular}{lrrrr} 
Model & $r(x, \hat{x})$ & $r\left(e_{x}, \hat{x}\right)$ & $r\left(e_{x}, \hat{\dot{x}}\right)$ & $r(\hat{\dot{x}}, \dot{x})$ \\
\hline Kano & 0.99976 & 0.1859 & 0.2424 & 0.8586 \\
Karnopp & 0.99996 & 0.1484 & -0.1621 & 0.9951 \\
LuGre & 0.99996 & 0.1285 & -0.1615 & 0.9947 \\
GMS & 0.99996 & 0.0543 & 0.0256 & 0.9945 \\
\hline
\end{tabular}

\begin{tabular}{lrrrr} 
Model & $r(x, \hat{x})$ val. & $r\left(e_{x}, \hat{x}\right)$ val. & $r\left(e_{x}, \hat{\dot{x}}\right)$ val. & $r(\hat{\dot{x}}, \dot{x})$ val. \\
\hline Kano & 0.99913 & 0.5905 & 0.0064 & 0.3700 \\
Karnopp & 0.99954 & 0.0345 & -0.2568 & 0.9901 \\
LuGre & 0.99954 & 0.0398 & -0.2544 & 0.9899 \\
GMS & 0.99955 & -0.0203 & -0.1599 & 0.9884
\end{tabular}

val.: indicates to a statistical quantity calculated using the validation dataset.

Source: from Author.

\subsubsection{Teflon Gasket Model Prediction Analysis}

The teflon gasket valve is used only for algorithm benchmark, to check that the proposed algorithms are able to estimate good models when the valve presents low friction index. Analogously to the graphite gasket valve, Figures 4.47 to 4.58 show the teflon gasket valve model prediction accuracy. The Kano model has higher residuals, whereas the other models presented visually similar residuals. The other models presented visually similar residuals and exhibited similar responses, apart from a few localized differences. Regarding the stem velocity prediction, all models are very accurate and present pretty similar results. 

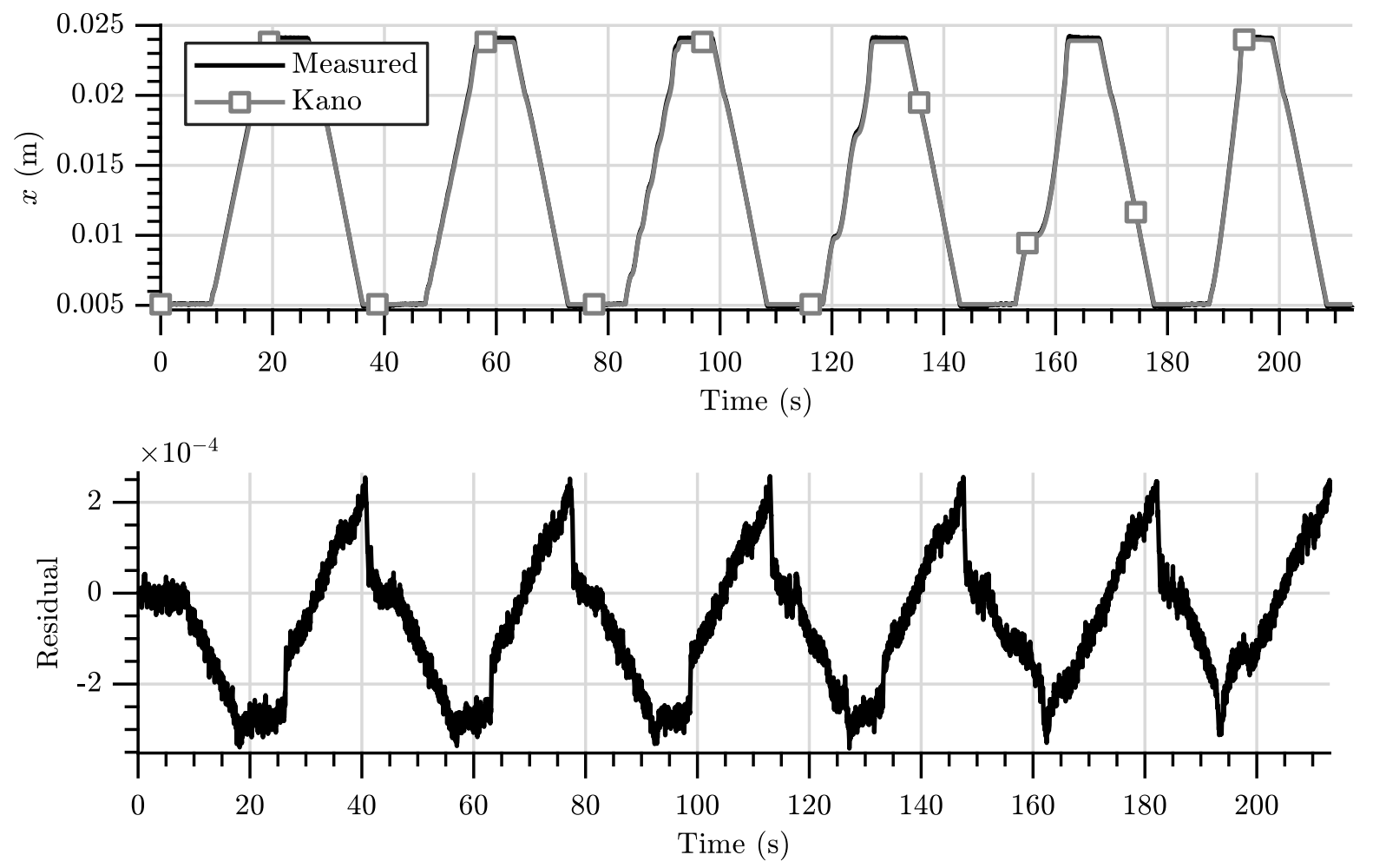

Figure 4.47 - Kano model teflon gasket valve residual using the estimation dataset.

Source: from Author.
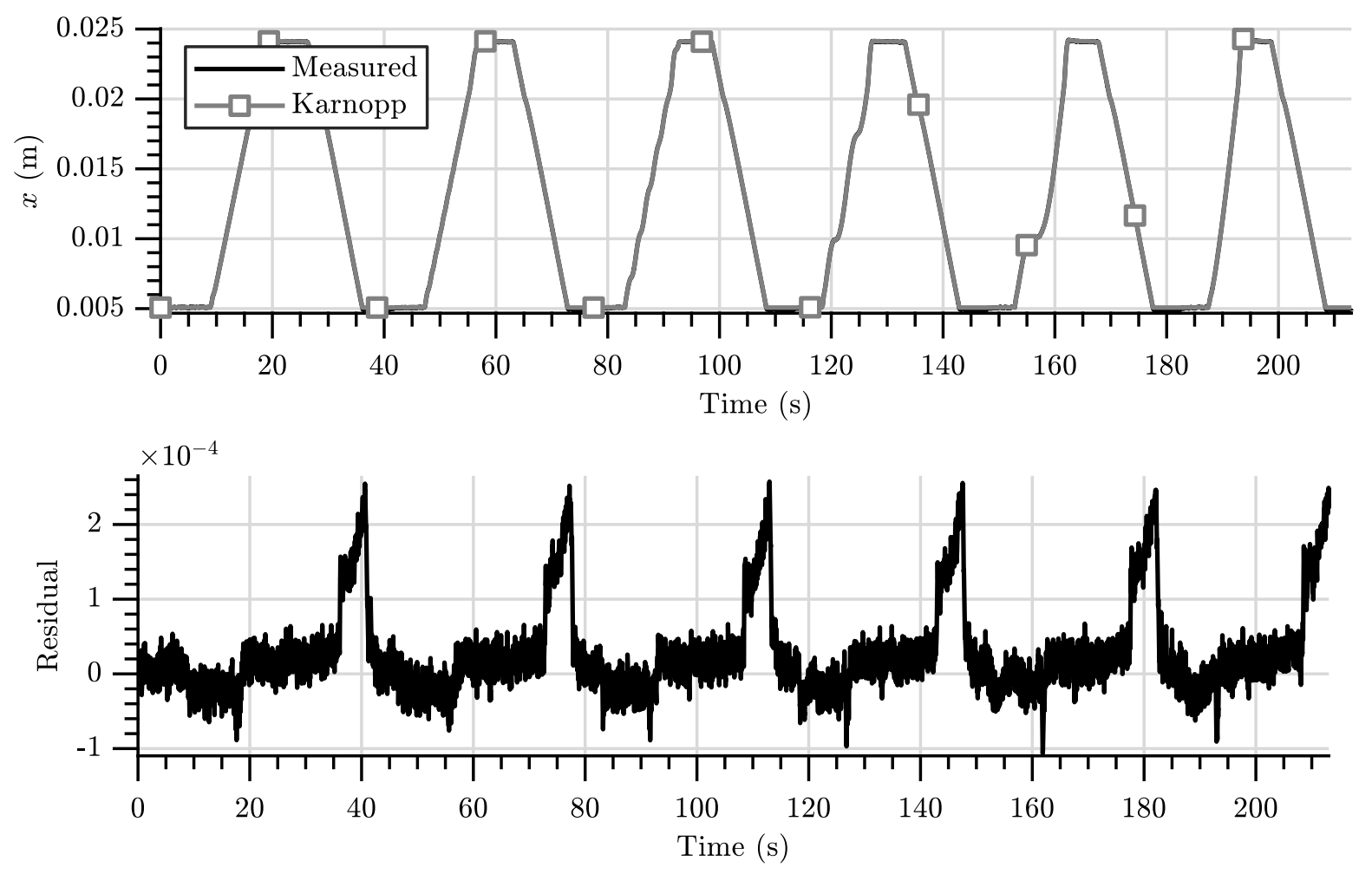

Figure 4.48 - Karnopp model teflon gasket valve residual using the estimation dataset.

Source: from Author. 

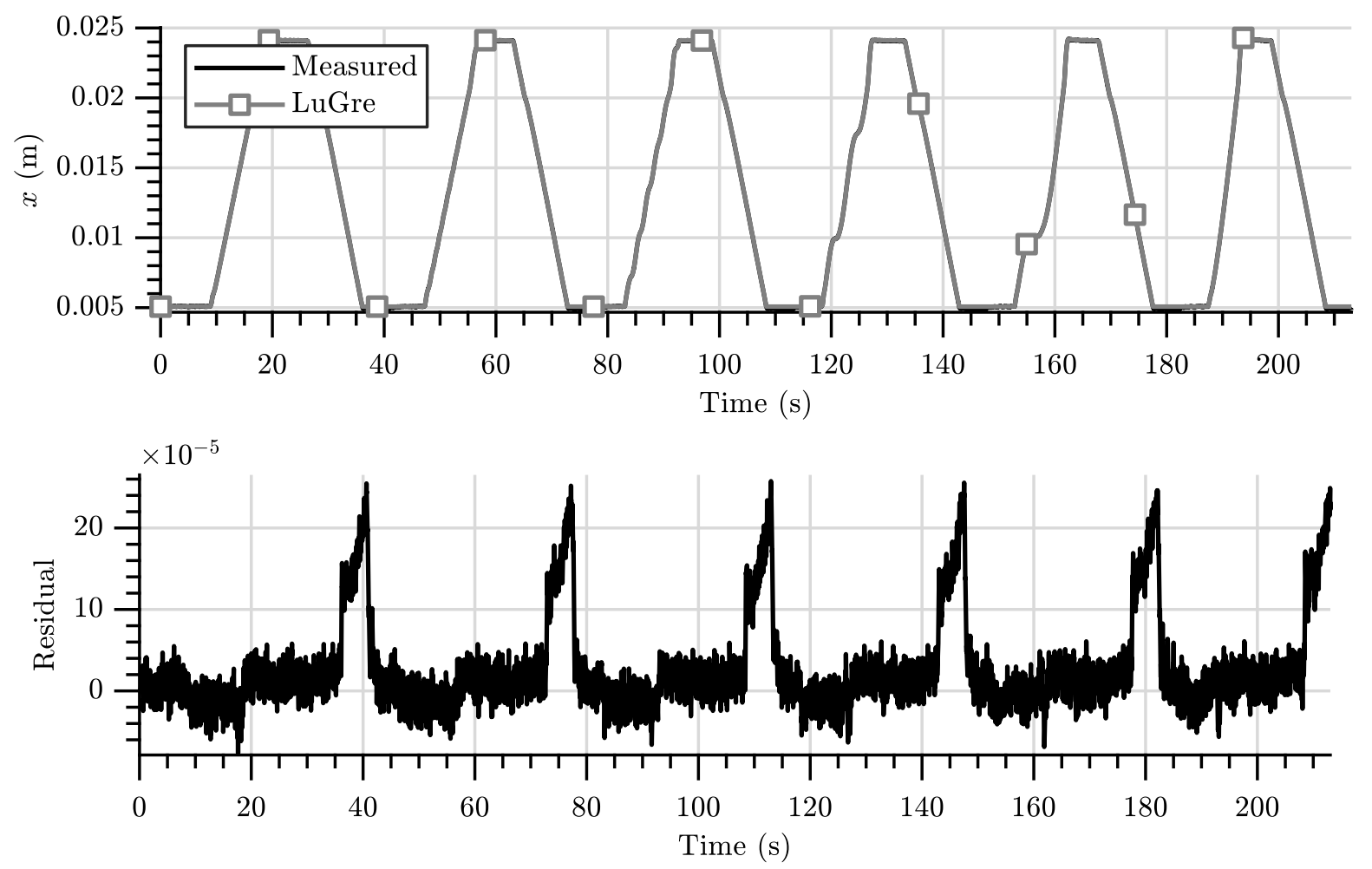

Figure 4.49 - LuGre model teflon gasket valve residual using the estimation dataset.

Source: from Author.
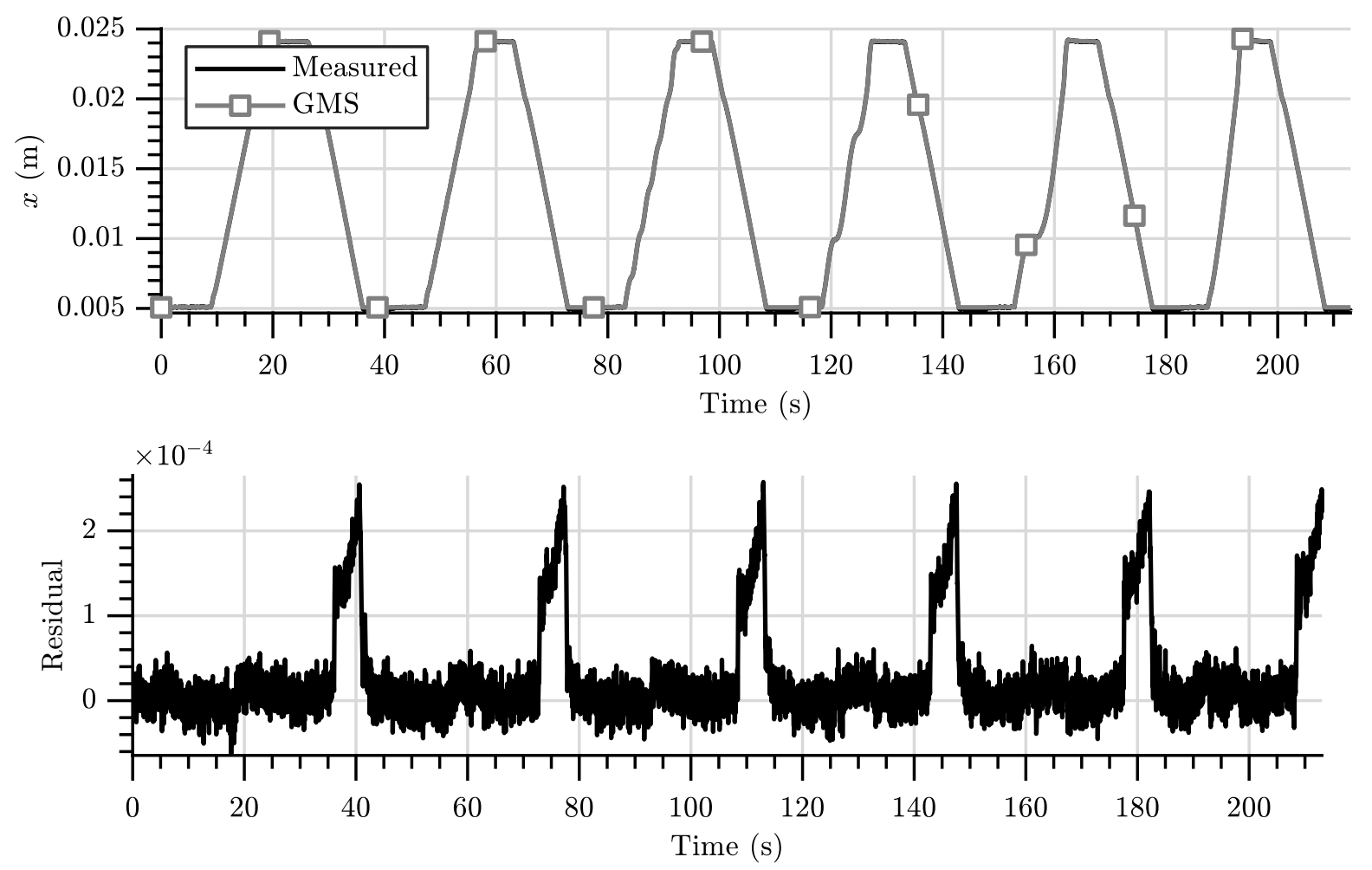

Figure 4.50 - GMS model teflon gasket valve residual using the estimation dataset. Source: from Author. 

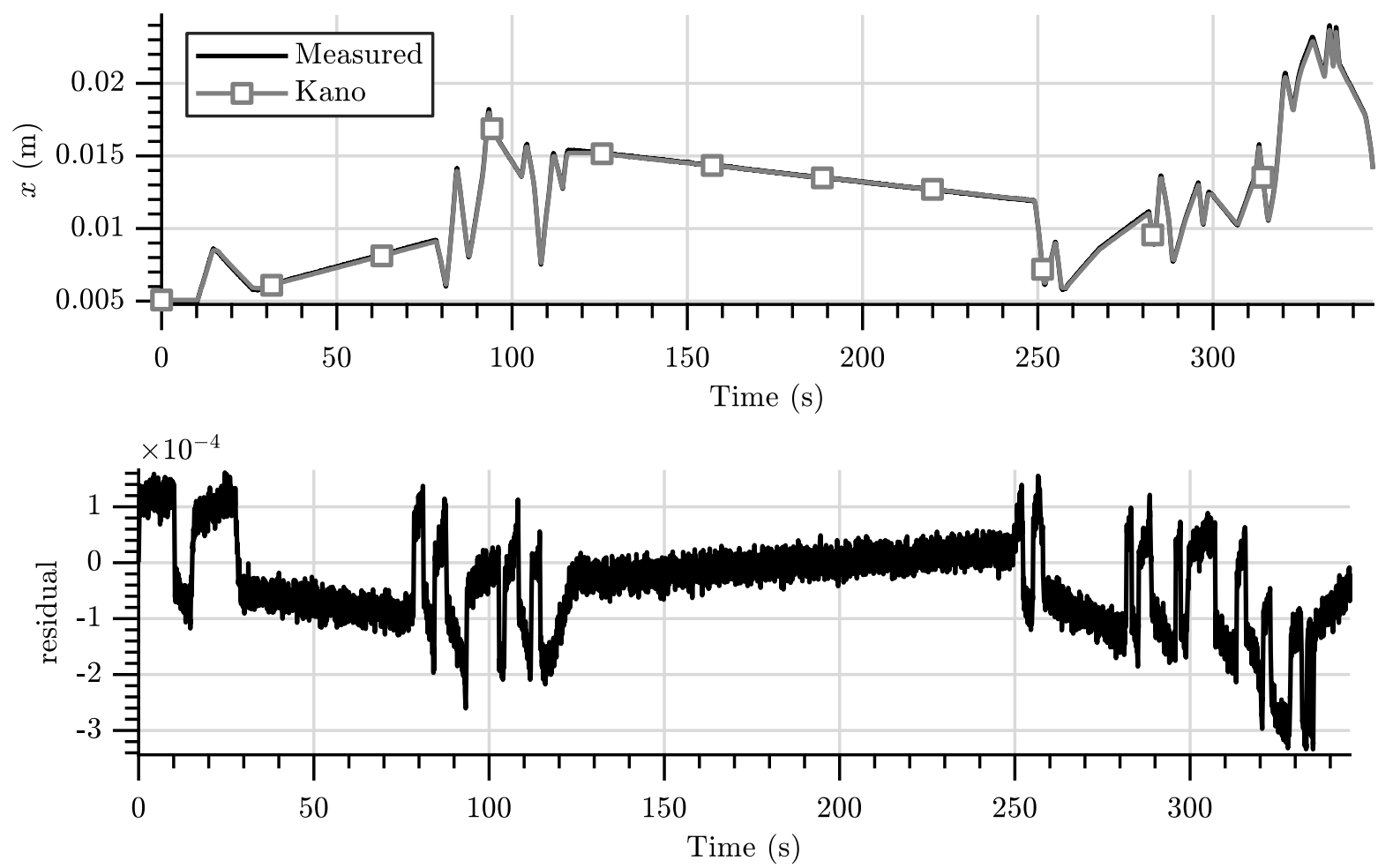

Figure 4.51 - Kano model teflon gasket valve residual using the validation dataset. Source: from Author.
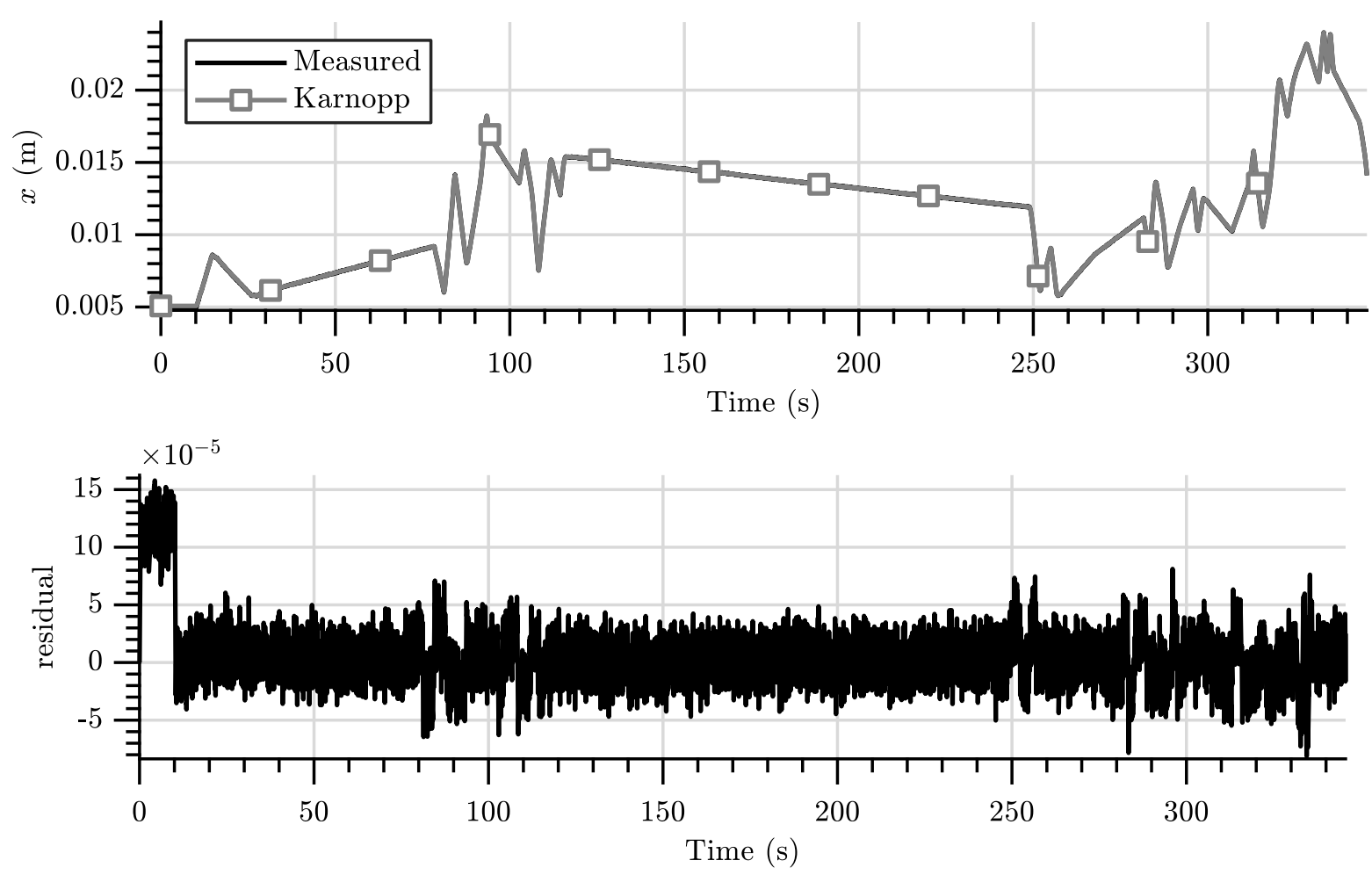

Figure 4.52 - Karnopp model teflon gasket valve residual using the validation dataset.

Source: from Author. 

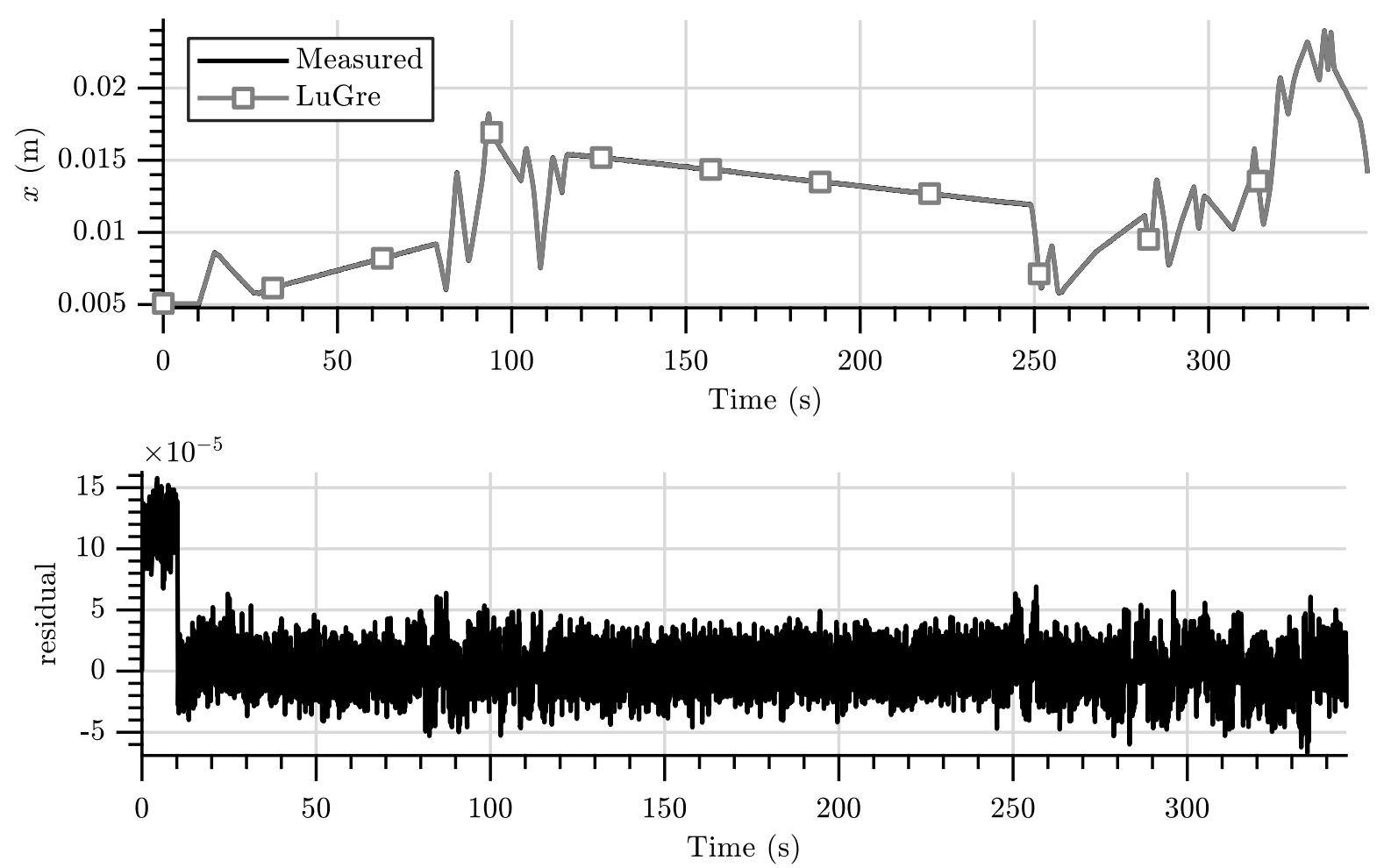

Figure 4.53 - LuGre model teflon gasket valve residual using the validation dataset.

Source: from Author.

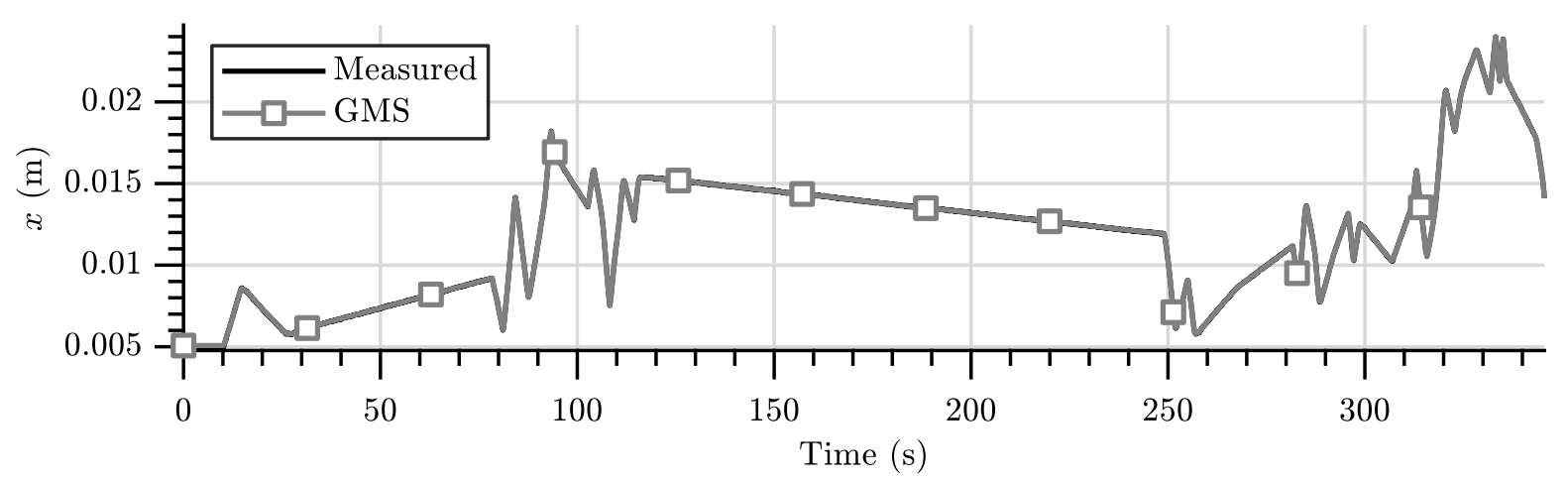

$\times 10^{-5}$

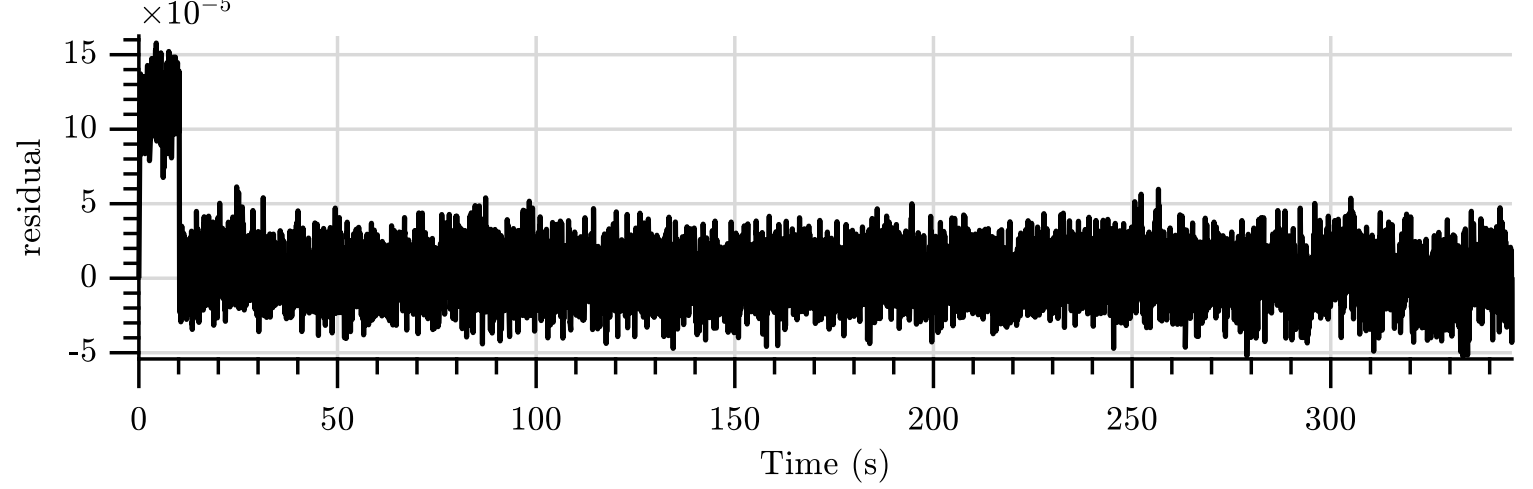

Figure 4.54 - GMS model teflon gasket valve residual using the validation dataset.

Source: from Author. 


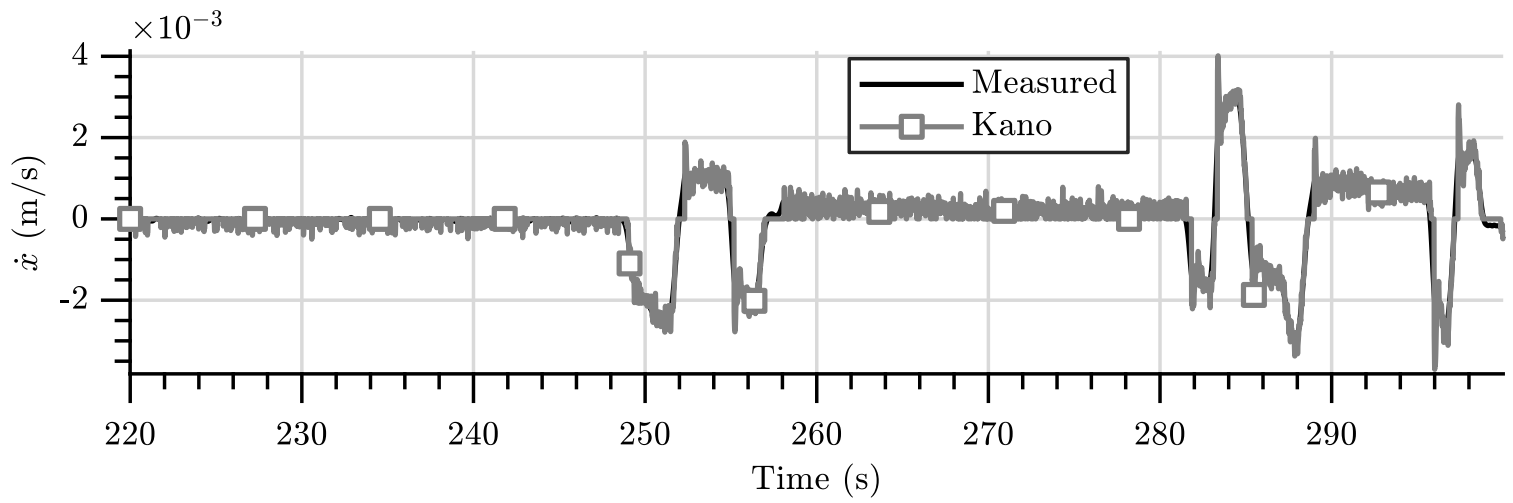

Figure 4.55 - Kano model teflon gasket valve stem velocity prediction (220s to $300 \mathrm{~s})$ using the validation dataset.

Source: from Author.

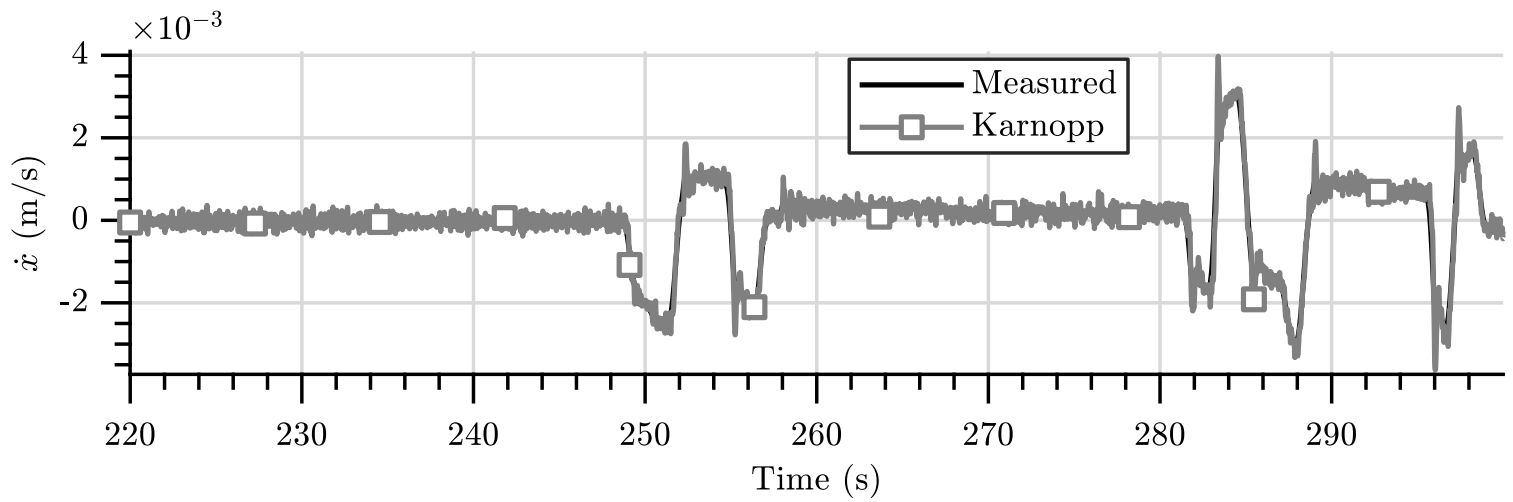

Figure 4.56 - Karnopp model teflon gasket valve stem velocity prediction (220s to $300 \mathrm{~s}$ ) using the validation dataset.

Source: from Author.

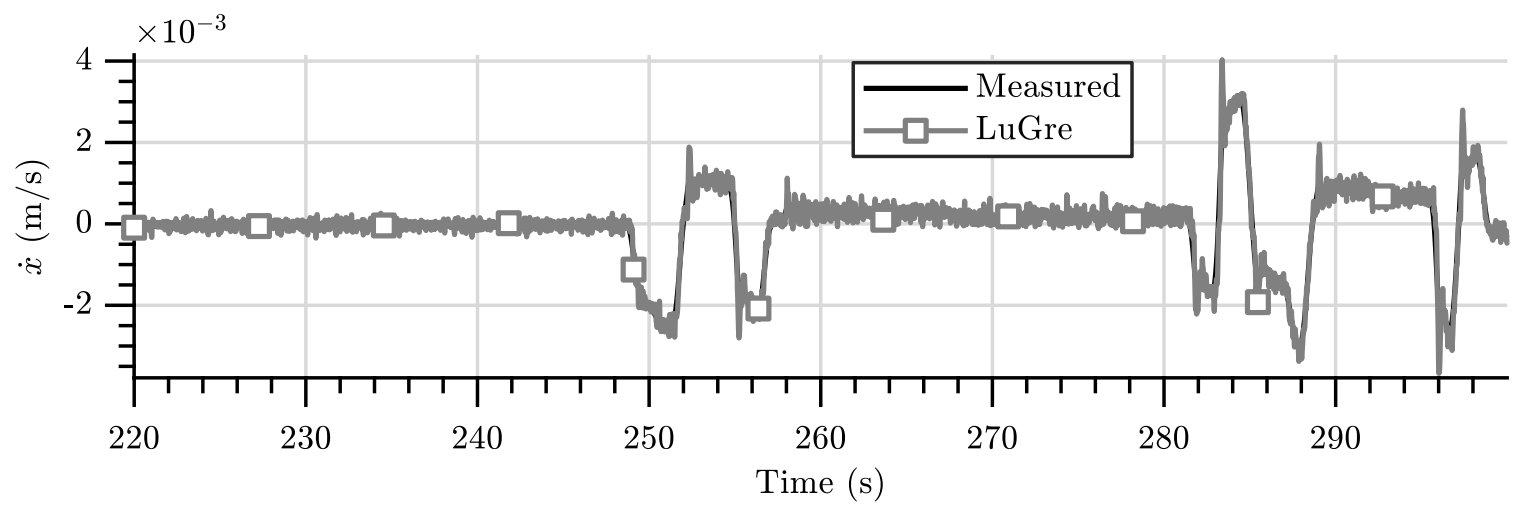

Figure 4.57 - LuGre model teflon gasket valve stem velocity prediction (220s to $300 \mathrm{~s})$ using the validation dataset.

Source: from Author. 


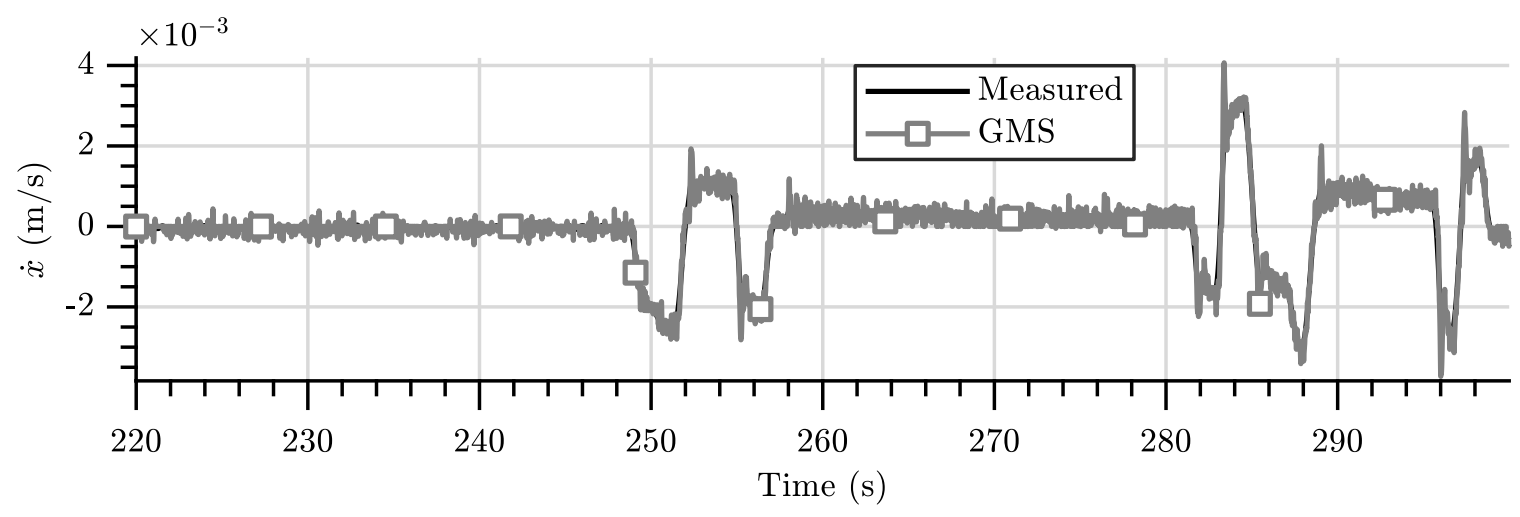

Figure 4.58 - GMS model teflon gasket valve stem velocity prediction (220s to $300 \mathrm{~s})$ using the validation dataset.

Source: from Author.

The error distribution is presented in Figures 4.59 and 4.60. The more well behaved residual distributions are provided by the Karnopp, LuGre and GMS models. Regarding the Kano model, the error distribution deviates from a Gaussian distribution, which indicates that the estimation can be improved. This deviation is likely to occur due to an estimation error regarding the $\hat{x}_{\min _{P}}$ and $\hat{x}_{\max _{P}}$ parameters, as the residual seems to be correlated to the stem position, as seen in Figures 4.47 and 4.51 .

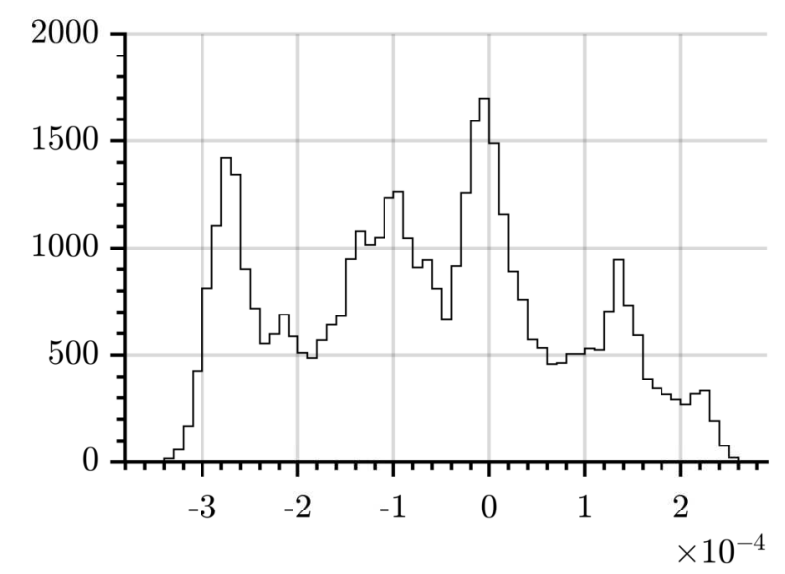

(a) Kano model.

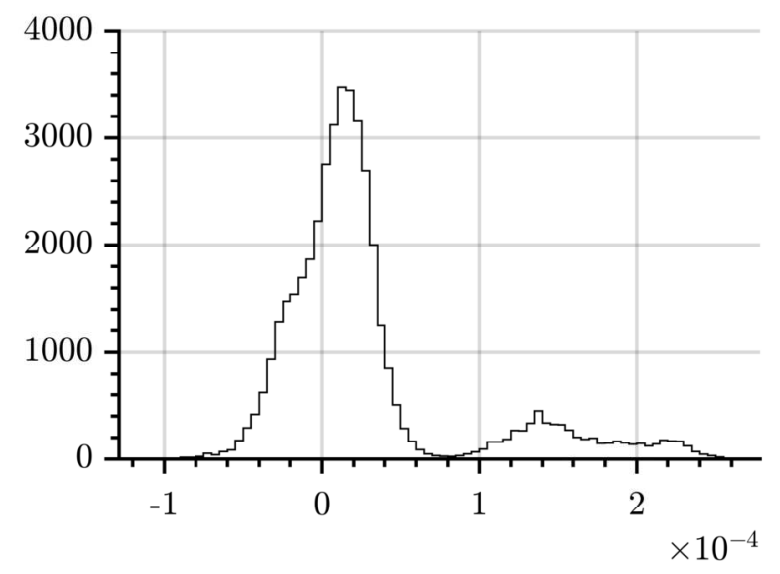

(b) Karnopp model.

Figure 4.59 - Kano and Karnopp teflon gasket valve prediction error distribution using the estimation dataset.

Source: from Author. 


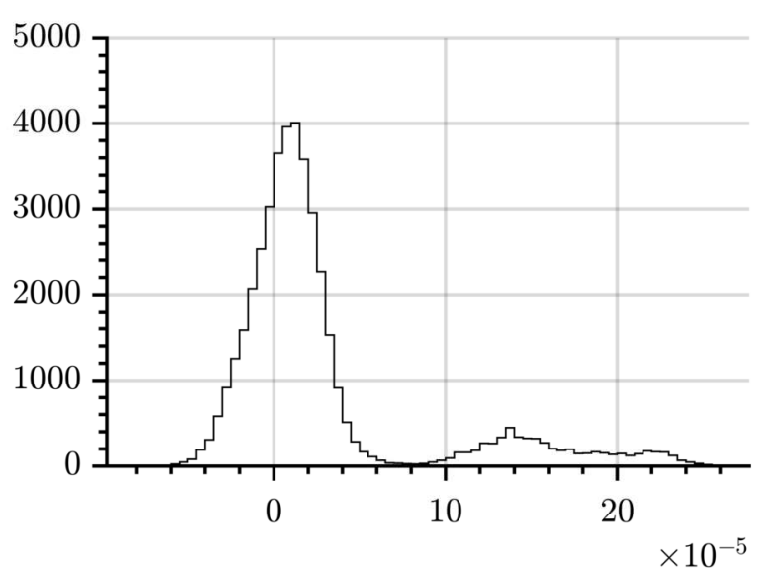

(a) LuGre model.

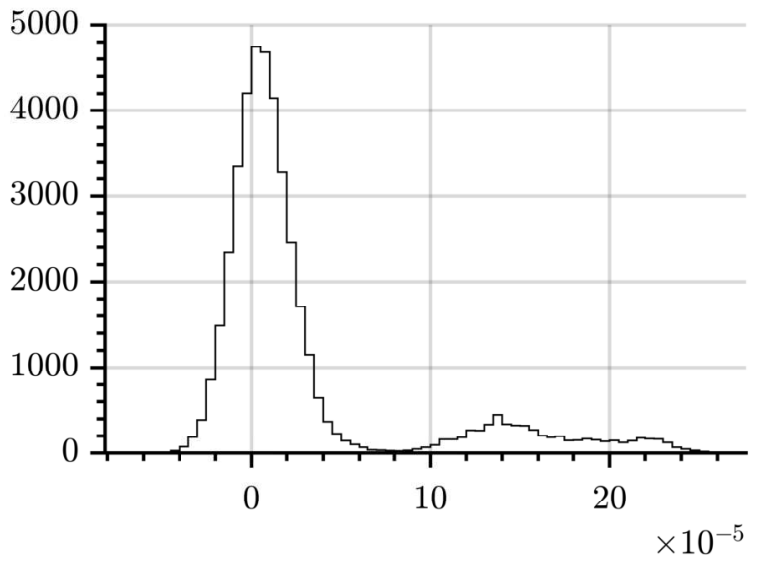

(b) GMS model.

Figure 4.60 - LuGre and GMS teflon gasket valve prediction error distribution using the estimation dataset.

Source: from Author.

The correlation coefficients between some variables of the teflon gasket valve models are presented in Table 4.32. The stem position and velocity prediction correlation coefficient is very high for every model and dataset, which is a good indicator of successful estimations. The Kano model, however, presented high correlation coefficients between residuals and the stem position, which indicates that the estimation could be improved (or that the model does not have a structure that is able to reproduce the plant perfectly).

Table 4.32 - Statistical measures of the teflon gasket valve prediction.

\begin{tabular}{lrrrr} 
Model & $r(x, \hat{x})$ & $r\left(e_{x}, \hat{x}\right)$ & $r\left(e_{x}, \hat{\dot{x}}\right)$ & $r(\hat{\dot{x}}, \dot{x})$ \\
\hline Kano & 0.99995 & -0.8456 & -0.3453 & 0.9909 \\
Karnopp & 0.99997 & -0.3886 & -0.2687 & 0.9911 \\
LuGre & 0.99998 & -0.3929 & -0.1670 & 0.9910 \\
GMS & 0.99998 & -0.3999 & -0.0288 & 0.9904 \\
\hline
\end{tabular}

\begin{tabular}{lrrrr} 
Model & $r(x, \hat{x})$ val. & $r\left(e_{x}, \hat{x}\right)$ val. & $r\left(e_{x}, \hat{\dot{x}}\right)$ val. & $r(\hat{\dot{x}}, \dot{x})$ val. \\
\hline Kano & 0.99983 & -0.3935 & -0.4853 & 0.9752 \\
Karnopp & 0.99998 & -0.3000 & -0.4050 & 0.9812 \\
LuGre & 0.99998 & -0.2750 & -0.2975 & 0.9821 \\
GMS & 0.99998 & -0.2995 & -0.1086 & 0.9808
\end{tabular}

val.: indicates to a statistical quantity calculated using the validation dataset.

Source: from Author. 


\section{Closed Loop Parameter Estimation}

This chapter is devoted to design algorithms to estimate the valve and friction parameters during the normal operation of valves with digital positioners, that is, the valve stem position is controlled by an embedded control system, whose stem position Set Point (SP) is given by the process Digital Control System (DCS).

Figure 5.1 presents a scheme for the diaphragm valve with a digital positioner, where $e x c$ is an external excitation signal that can be used to estimate the valve and friction parameters, $C_{p}$ is the stem position controller and $C_{H}$ is the hydraulic system controller, that is implemented in the DCS.

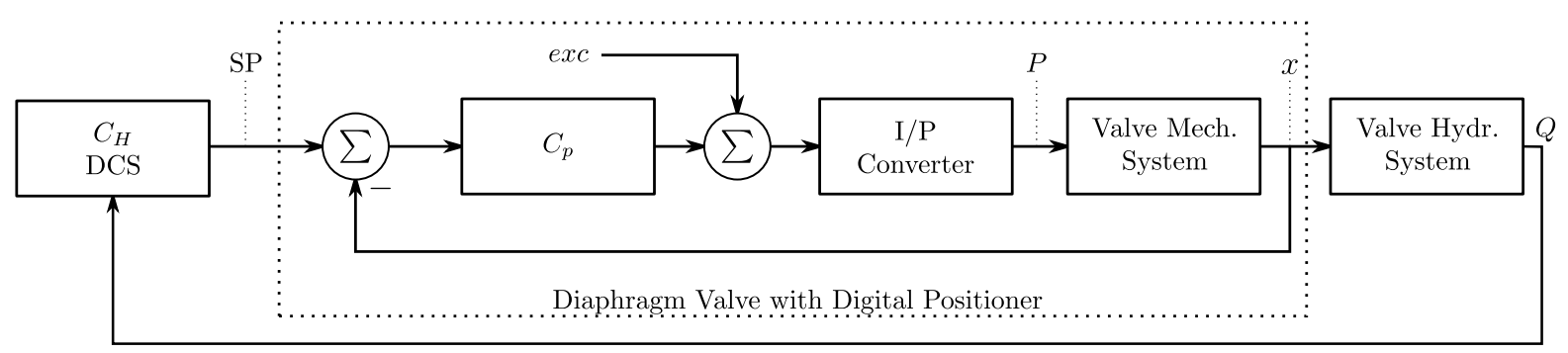

Figure 5.1 - Scheme of the diaphragm valve with digital positioner.

Source: from Author.

In this case, the valve stem is not able to move freely for obtaining the parameter estimates, which increases the estimation difficulty. Solving Eq. (4.5) also is a solution for the closed loop case. However, as in the open loop case, this optimization dimension is large, which demands studying possible simplifications.

\subsection{Valve Diagnostics and Friction Detection}

Assuming that the valve is delivered by the maintenance personal or the valve manufacturer with a good model, estimated in open loop, then identifying problems in the valve is a matter of comparing the measured and simulated signals, as presented in Figure 5.2 . 


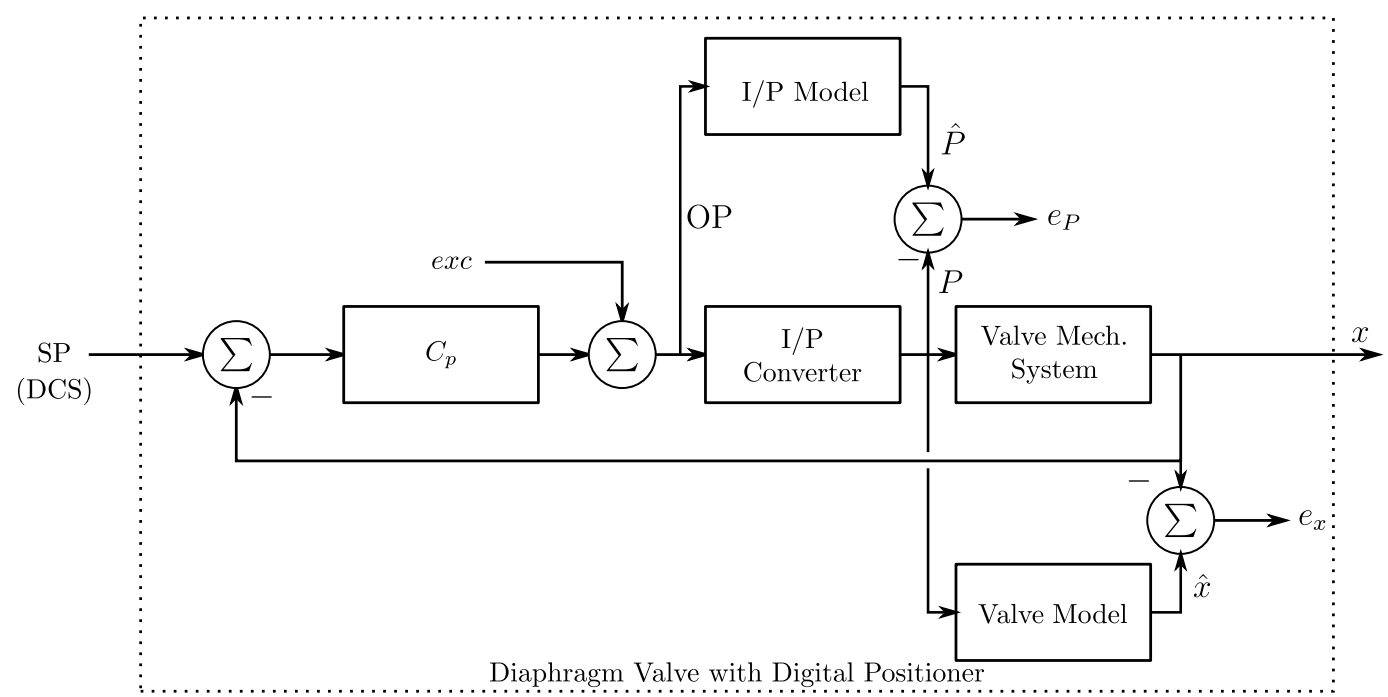

Figure 5.2 - Scheme of valve diagnostics by analyzing the difference between measured and simulated variables.

Source: from Author.

Having the standard $f$ it index for the I/P converter and the valve models, obtained during the valve maintenance procedure or by the manufacturer, the valve signals can be stored in a memory with a long time window, e.g. 20 minutes, and the actual $f$ it indexes can be compared to the standard ones. If a deviation occurs, then a problem in the valve is detected, and it is possible to define if it is in the I/P converter or in the valve mechanical system.

When a problem is detected in the I/P converter or the valve mechanical system, then it is possible to reinitialize the model to check if the problem is related to the model simulation. To do so, one may notice that the models have internal states that have to be initialized accordingly to the valve. This may be achieved by switching $C_{p}$ to manual, increasing $\mathrm{OP}$ so just the stem position increases and waiting $5 \cdot \tau_{\mathrm{I} / \mathrm{P}_{s}} \mathrm{~s}$, to restart the model simulation with new initial conditions and switch the controller to automatic again. In this case, it is possible to determine all initial model states, since the valve is in the presliding state with constant position and zero velocity. For all models, the initial stem position used to restart the simulation is obviously the valve measured position immediately before the controller is switched to automatic again. For the Kano model, it is also necessary to obtain the input when the valve stopped moving, OPs from Algorithm 3.1 and the direction the valve was moving before the stem stopped, which is obviously upward $(\mathrm{d}=1)$. The other first principle models need to be informed of the initial stem velocity, which is zero. Finally, the LuGre and GMS models need the initial internal state position, which is calculated as in Eqs. (4.30) and (4.33), but with reversed signal. The I/P model only needs to be informed of the initial diaphragm pressure, $P[0]$. At every moment during this procedure, the controller logic should switch back to automatic if the DCS SP varies drastically, to avoid affecting the control loop performance. 
If even after restarting the model and evaluating the $f i t$ between measured and simulated variables are still high, then it is likely to have a problem with the $\mathrm{I} / \mathrm{P}$ converter or the valve mechanical system.

As for a problem with the I/P converter, it should not influence the valve mechanical model, as it uses the measured diaphragm pressure as input. However, it is possible to estimate the $\mathrm{I} / \mathrm{P}$ model parameters again and refine the $C_{p}$ tuning. Estimating the $\mathrm{I} / \mathrm{P}$ parameters in closed loop is addressed in Section 5.2 and updating the valve controller is discussed in the next chapter.

The stem position residual can be evaluated as well, to detect defects. As a test, consider the plant as a valve with GMS friction model simulation in closed loop, with model parameters as presented in Tables 3.1 and 3.2, unless said otherwise, controlled by a parallel Proportional Integral Derivative Controller (PID) with $K_{p}=0.72, K_{i}=0.43 \mathrm{rep} / \mathrm{s}$, $K_{d}=6.74 \mathrm{~s}$ and derivative filter time constant $\tau_{d f}=278 \mathrm{~s}$. Then, the signals generated by this plant are used to excite the valve model, with the same parameters as in Tables 3.1 and 3.2. Figure 5.3a shows the residual distribution of a valve with factory performance, i.e. the plant and the model have the same parameters and structure, so the residual can be considered a Gaussian random variable, due to the measurement noise. Figures 5.3b, $5.4 \mathrm{a}$ and $5.4 \mathrm{~b}$ present the test cases when the plant has a problem with a $10 \%$ increase in $k, 10 \%$ increase in $F_{\text {init }}$ and increase in several friction parameters. It is possible to notice that when a deviation occurs, the most apparent modification in the residual distribution is a mean value drift.

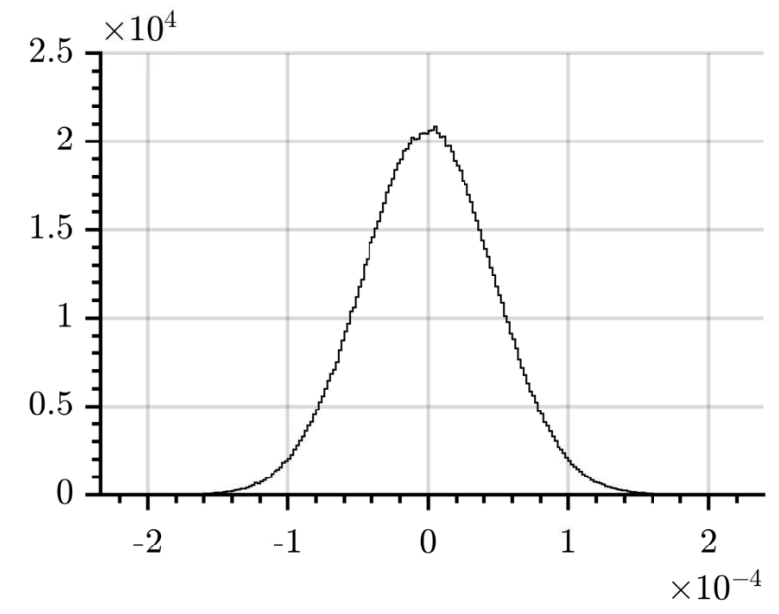

(a) Valve with factory performance.

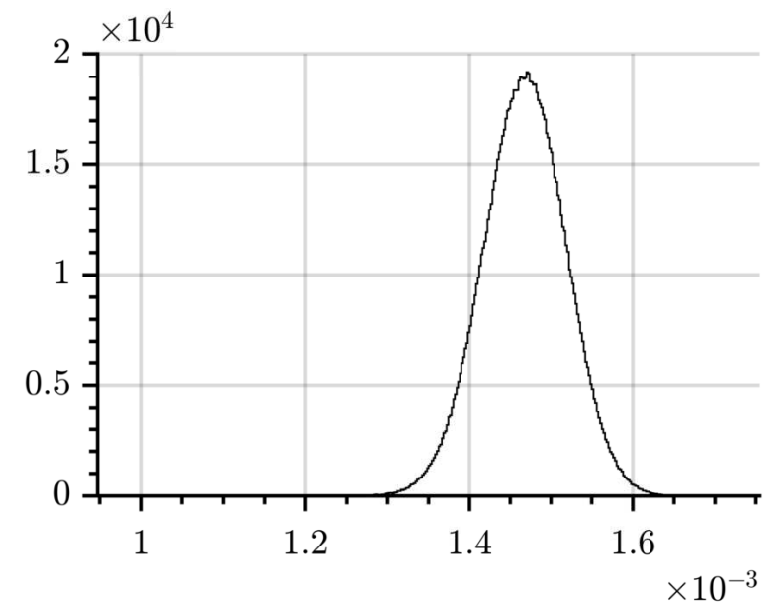

(b) $10 \%$ Increase in $k$ plant parameter.

Figure 5.3 - Residual distribution for plant with factory performance and deviation in $k$.

Source: from Author. 


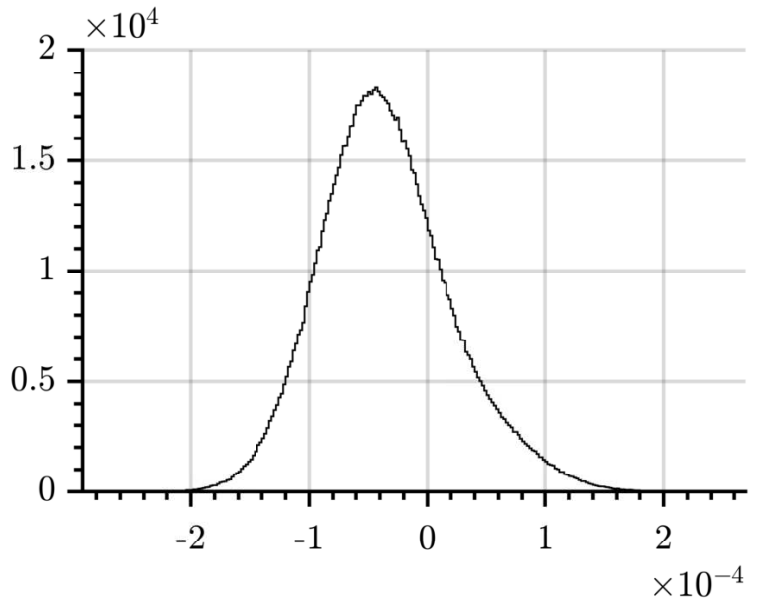

(a) $10 \%$ Increase in $F_{\text {init }}$ plant parameter.

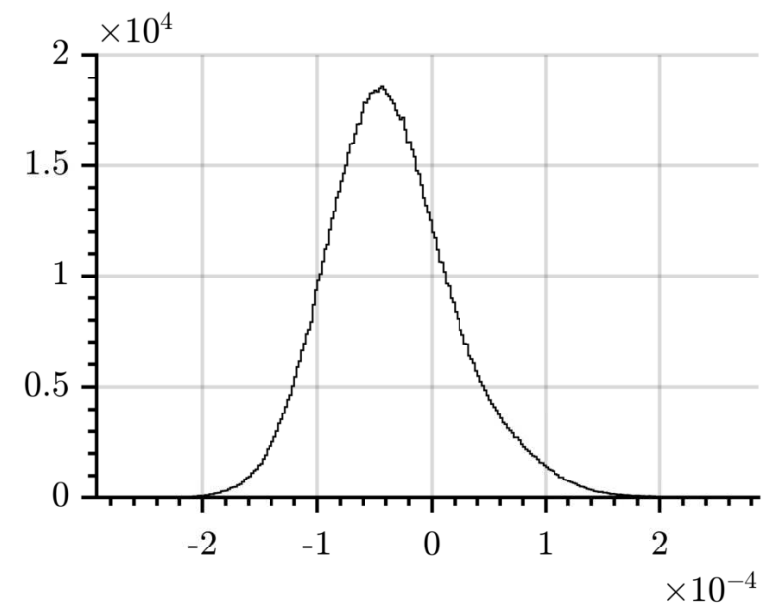

(b) Deviation of $-10 \%$ in $F_{c}, 10 \%$ in $F_{s}, 20 \%$ in $F_{v}$ and $20 \%$ in $v_{s}$ in plant friction parameters.

Figure 5.4 - Residual distribution for plant with deviation in $F_{\text {init }}$ and friction parameters.

Source: from Author.

Table 5.1 presents some statistical measures of the cases of test residuals. When there is a deviation in $k$, the mean value drifts from the ideal case, as well as the correlations between residual and simulated stem position and velocity. Besides these three parameters deviation, when $F_{\text {init }}$ deviates, then there is an increase in the distribution standard deviation and skewness as well, $\mu_{3}\left(e_{x}\right)$, which is defined as:

$$
\mu_{3}\left(e_{x}\right)=\frac{1}{n_{f}} \sum_{n=1}^{n_{f}}\left(e_{x}[n]-\mu\left(e_{x}\right)\right)^{3} .
$$

The friction deviation may affect every statistical measure, depending on which parameter is affected, but in general it should have a less strong correlation between residuals and the stem position, since friction is independent of $x$. Therefore, these statistical measures may be calculated periodically and if drifts are detected, then it may be used to classify which problem is occurring in the valve mechanical system.

Table 5.1 - Statistical measures of simulated defective valves of Figures 5.3a to $5.4 \mathrm{~b}$.

\begin{tabular}{lrrrrr} 
Case & $\mu\left(e_{x}\right)$ & $\operatorname{std}\left(e_{x}\right)$ & $\mu_{3}\left(e_{x}\right)$ & $r\left(e_{x}, \hat{x}\right)$ & $r\left(e_{x}, \hat{\dot{x}}\right)$ \\
\hline No defect & $2.2 \cdot 10^{-8}$ & $4.6 \cdot 10^{-5}$ & $4.6 \cdot 10^{-16}$ & 0.0044 & -0.0058 \\
$k$ deviation & 0.0015 & $9.5 \cdot 10^{-5}$ & $-8.4 \cdot 10^{-12}$ & 0.8707 & -0.6323 \\
$F_{\text {init deviation }}$ & 0.0009 & 0.0012 & $7.9 \cdot 10^{-9}$ & 0.9378 & -0.4519 \\
Friction deviation & $-3.4 \cdot 10^{-5}$ & $5.9 \cdot 10^{-5}$ & $1.8 \cdot 10^{-13}$ & -0.2267 & 0.3102 \\
\hline
\end{tabular}

Source: from Author.

Another measure to determine if the valve mechanical system has deviated, is the valve deadband. If it has changed, when compared to its standard value, then it is 
likely that the friction has deviated over time. Estimating the valve deadband can be accomplished by noting that the valve signature curve tends to present a trapezoidal shape, as already noted by Kano et al. (2004), Choudhury et al. (2006). If two lines are fitted to the valve operational data containing only nonzero stem velocities, then the distance between these lines corresponds to the valve deadband. The procedure for obtaining a $S_{0}$ estimation is presented in Algorithm 5.1. The estimated parameters are calculated easily, with minimal valve deviation from the desired position SP, and may be important for defining which parameters are responsible for the valve performance measure deviation and designing excitation signals.

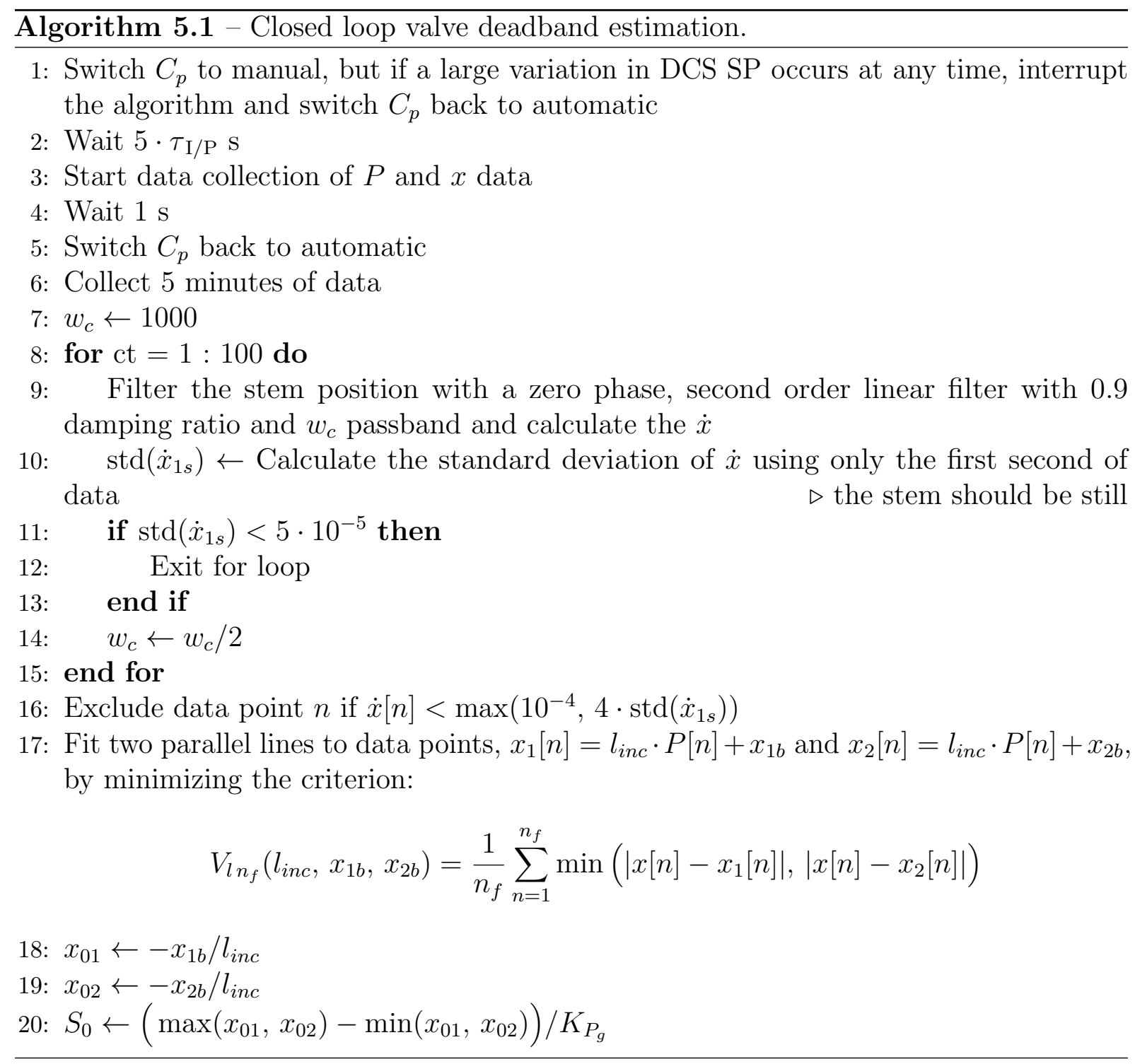

Algorithm 5.2 presents a conceptual framework to detect defects in the valve and start the necessary procedures to estimate new model parameters and update $C_{p}$. If the Kano model is used, then the algorithm should be changed to estimate $x_{\min \mathrm{P}}$ and $x_{\max \mathrm{P}}$, instead of $k$ and $F_{\text {init }}$. 


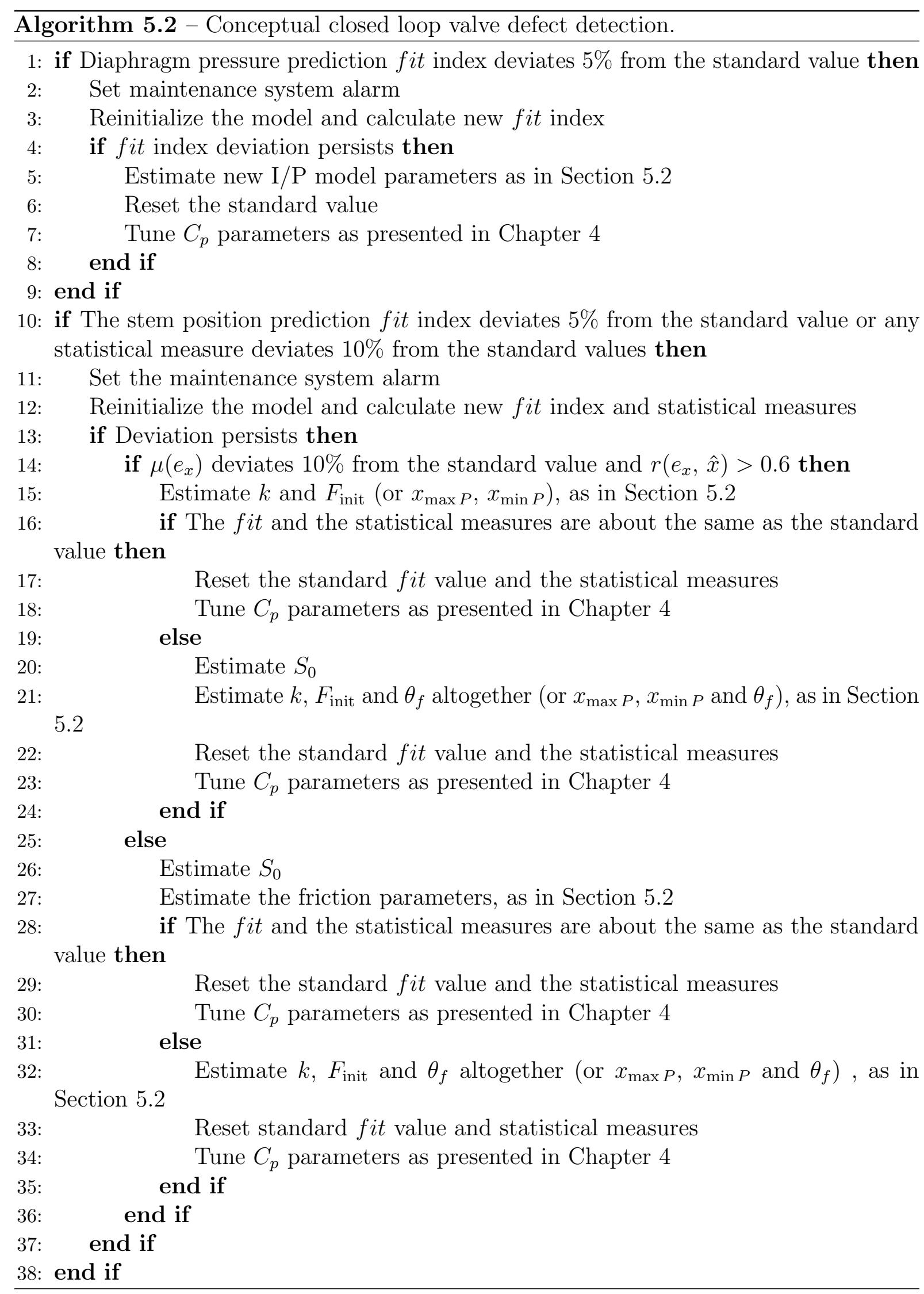




\subsection{Closed Loop Parameter Estimation}

To estimate the I/P converter parameters, it is enough to apply a rectangular excitation signal in exc, with amplitude $1.5 \cdot S_{0}$ and $80 \mathrm{~s}$ period, very similar to the open loop case. Of course, the exc signal will be highly attenuated by $C_{p}$, which is desirable, so to decrease the perturbations in the control loop. However, as the overall signal is not rectangular and the $\mathrm{I} / \mathrm{P}$ converter has nonlinear dynamics, it is not expected that the parameters will be the same as in the open loop estimation, but it is enough to accommodate defective or drifting I/P converter dynamics. The parameters can be estimated exactly as described in Section 4.1.

As the valve and the friction parameter estimation is carried out with an infinite steps ahead simulation of the valve, once again the biggest challenge is to determine the initial states of the valve to initialize the simulation. This can be accomplished with the exact procedure of simulation reinitialization from Section 5.1. After $C_{p}$ is switched to automatic again, the excitation signal exc can be started, as well as the estimation data collection.

With respect to the excitation signal design, the most important aspect for estimating friction is to collect data from a wide range of stem velocities and from presliding and sliding states. This may not be informative enough with just the SP excitation from DCS, therefore the need for the perturbation signal exc. The suggested exc is an aleatory velocity ramp signal, with range from $10^{-5}$ to $3 \cdot 10^{-3} \mathrm{~m} / \mathrm{s}$ and aleatory magnitude from 0.7 to 1.2 times $S_{0}$. The exc signal is corrected by $C_{p}$, so there are no large disturbances in the stem position, which is desirable for maintaining the process controlled, but it is disadvantageous for obtaining valve information. However, it should be enough to obtain fair estimates to update the model if the valve behavior varies with time. Figure 5.5 presents an example of excitation signal with $S_{0}=25.7$.

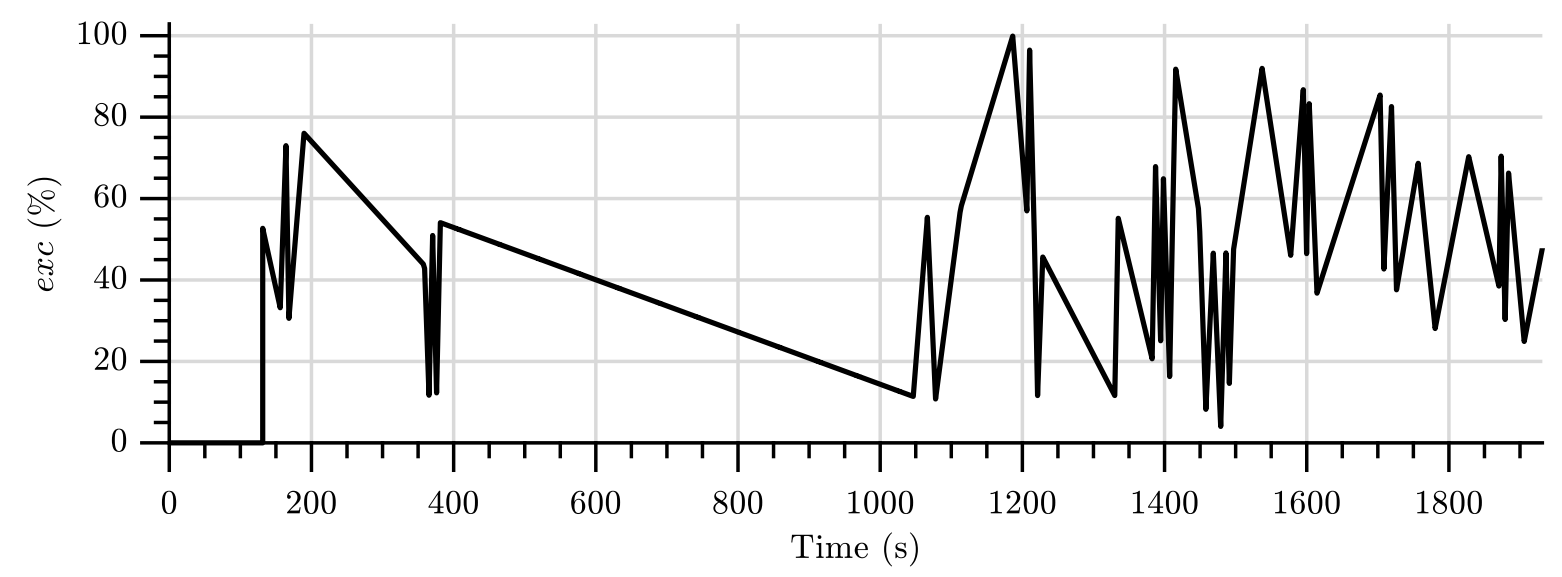

Figure 5.5 - Closed loop excitation signal example.

Source: from Author. 
To estimate $k$ and $F_{\text {init }}$ only, the residual function to be minimized is:

$$
\left\{\hat{k}, \hat{F}_{\text {init }}\right\}=\underset{k, F_{\text {init }}}{\arg \min } \sqrt{\sum_{n=1}^{n_{f}}\left(x[n]-\hat{x}\left(n, \mathrm{OP}[n], x[0], \hat{m}, k, F_{\text {init }}, \hat{\theta}_{f}\right)\right)^{2}} .
$$

To estimate the friction parameters, the residual function is exactly as in Eq. (4.17). Finally, to estimate all parameters it is just a matter of augmenting Eq. (4.17) with $k$ and $F_{\text {init }}$, as suggested in the open loop case. If the Kano model is used in the estimation, then it is necessary to augment Eq. (4.17) with $x_{\max P}$ and $x_{\min P}$, instead.

The search space and penalty functions for the friction parameters can be calculated as described in Section 4.2, using the closed loop $S_{0}$ estimate. The $k$ and $F_{\text {init }}$ search space should be created by allowing a deviation of $\pm 50 \%$ from the previous estimate, which is a very conservative criterion. Regarding the Kano model, the $x_{\max P}$ and $x_{\min P}$ also should be allowed to vary $\pm 50 \%$ from the previous value. To solve the estimation problem, the GDES optimization method is used and the measured variables filtering should be addressed as in the open loop case.

When estimating parameters in closed loop, there is a problem with parameter bias due to correlation between the measurement noise and the system input. This problem is addressed in the following section.

\subsection{Stochastic Parameters Estimation Analysis}

In this section, the residual function stochastic analysis is performed to ensure that the expected values for the estimated parameters are equal to the plant parameters.

The first principle valve model is given by the following difference equation, as seen in Section 3.2:

$$
x[n]=\frac{T_{s}^{2}}{4} \frac{q^{-1}+2 q^{-2}+q^{-3}}{1-2 q^{-1}+q^{-2}} \frac{F_{\text {res }}[n]}{m} .
$$

Expanding the term $F_{\text {res }}$, multiplying both sides by the denominator and rearranging, one obtains:

$$
\begin{aligned}
4 x[n]+\left(\frac{T_{s}^{2} k}{m}-8\right) x[n-1]+ & \left(4+\frac{2 T_{s}^{2} k}{m}\right) x[n-2]+\frac{T_{s}^{2} k}{m} x[n-3]= \\
& \frac{T_{s}^{2}}{m}\left(q^{-1}+2 q^{-2}+q^{-3}\right)\left(S_{a} P[n]-F_{f}[n]-F_{\text {init }}\right) .
\end{aligned}
$$

For a further analysis, it is necessary to expand the term $F_{f}[n]$ for each friction model, which is done in the following sections.

\subsubsection{Karnopp Friction Model}

The Karnopp model calculates the friction force using three equations, that are switched depending on the velocity and resultant force of the system. If the stem velocity 
is zero and $\left|F_{r}[n]\right| \leq F_{s}$, the friction force is:

$$
F_{f}[n]=S_{a} P[n]-k x[n]-F_{\text {init }}
$$

Substituting Eq. (5.5) into Eq. (5.4) and simplifying it, the stem position equation is reduced to:

$$
x[n]=2 x[n-1]-x[n-2] .
$$

However, as in this case the stem velocity is forced to zero, the valve equation is simply:

$$
x[n]=x[n-1],
$$

which means that the stem position does not change.

If the stem velocity is zero and $\left|F_{r}[n]\right|>F_{s}$, then:

$$
F_{f}[n]=F_{s}
$$

Substituting Eq. (5.8) into Eq. (5.4) and rearranging the terms, the valve model is reduced to:

$$
x[n]=\left(\frac{T_{s}^{2}}{4 m}\right) \frac{q^{-1}+2 q^{-2}+q^{-3}}{1+\left(\frac{T_{s}{ }^{2} k}{4 m}-2\right) q^{-1}+\left(1+\frac{T_{s}{ }^{2} k}{2 m}\right) q^{-2}+\frac{T_{s}{ }^{2} k}{4 m} q^{-3}}\left(S_{a} P[n]-F_{s}-F_{\text {init }}\right) .
$$

Note that Eq. (5.9) represents a mechanical system composed of mass and spring and therefore the linear discrete equation tends to be marginally stable, as no damping is active. This equation is used only in one time step each time the valve switches from sliding to presliding state.

Lastly, if the stem velocity is different from zero:

$$
F_{f}[n]=\left[F_{c}+\left(F_{s}-F_{c}\right) \exp \left(-\left(\dot{x}[n] / v_{s}\right)^{2}\right)\right] \operatorname{sgn}(\dot{x}[n])+F_{v} \dot{x}[n] .
$$

Using the concept of Generalized Gradient Theorem (CLARKE et al., 1998), that states that if a function is Lipschitz near the discontinuous point and it is defined over domain subsets, such that the union of these subsets spans the real space, and the intersection of these subsets is null, then the function is differentiable and its derivative lies anywhere in the convex hull formed by the gradient of the function at every subset limit, it is possible to calculate the first order partial differential equation of Eq. (5.10), with respect to $\dot{x}[n]$, around the stem velocity $\dot{x}_{L}$ :

$$
F_{L}\left(\dot{x}_{L}\right)=\left.\frac{\partial F_{f}[n]}{\partial \dot{x}[n]}\right|_{\dot{x}[n]=\dot{x}_{L}}=-2\left(F_{s}-F_{c}\right) \exp \left(-\left(\dot{x}_{L} / v_{s}\right)^{2}\right) \frac{\dot{x}_{i}}{v_{s}^{2}} \operatorname{sgn}\left(\dot{x}_{L}\right)+F_{v} .
$$


Note that the generalized derivative of the signal function is zero. The valve model with linearized friction force model is:

$$
\begin{aligned}
& x[n]\left[4+\left(\frac{T_{s}^{2} k}{m}-8\right) q^{-1}+\left(4+\frac{2 T_{s}^{2} k}{m}\right) q^{-2}+\frac{T_{s}^{2} k}{m} q^{-3}\right]= \\
& \frac{T_{s}^{2}}{m}\left(q^{-1}+2 q^{-2}+q^{-3}\right)\left(S_{a} P[n]-F_{d}\left(\dot{x}_{L}\right)-F_{L}\left(\dot{x}_{L}\right) \frac{2}{T_{s}} \frac{1-q^{-1}}{1+q^{-1}} x[n]-F_{\text {init }}\right) .
\end{aligned}
$$

Rearranging the terms, Eq. (5.12) is rewritten as:

$$
\begin{aligned}
x[n]\left[4+A_{L K 1}\left(\dot{x}_{L}\right) q^{-1}+A_{L K 2} q^{-2}+A_{L K 3}\left(\dot{x}_{L}\right) q^{-3}\right] & = \\
\frac{T_{s}^{2}}{m}\left(q^{-1}+2 q^{-2}+q^{-3}\right) & \left(S_{a} P[n]-F_{d}\left(\dot{x}_{L}\right)-F_{\text {init }}\right),
\end{aligned}
$$

with:

$$
\begin{aligned}
A_{L K 1}\left(\dot{x}_{L}\right) & =\frac{T_{s}^{2} k}{m}+\frac{2 T_{s}}{m} F_{L}\left(\dot{x}_{L}\right)-8 \\
A_{L K 2} & =4+\frac{2 T_{s}^{2} k}{m} \\
A_{L K 3}\left(\dot{x}_{L}\right) & =\frac{T_{s}^{2} k}{m}-\frac{2 T_{s}}{m} F_{L}\left(\dot{x}_{L}\right) .
\end{aligned}
$$

It is clear from Eqs. (5.9) and (5.13) that the valve model, when in sliding state or in presliding with $\left|F_{r}[n]\right|>F_{s}$, can be approximated by a transfer function with variable parameters, given the linearization point:

$$
x[n]=G_{L K}\left(q, \dot{x}_{L}\right)\left(S_{a} P[n]-F_{d}\left(\dot{x}_{L}\right)-F_{\text {init }}\right) .
$$

As already mentioned in Chapter 4, the valve model with Karnopp friction model is a filter with variable gain and poles, given the stem velocity. The friction force decreases or increases the overall system damping when the stem velocity is lower, higher than the Stribeck velocity, respectively.

\subsubsection{LuGre Friction Model}

Given Eq. (3.9), the LuGre model friction force is:

$$
F_{f_{L}}[n]=\sigma_{0} z[n]+\sigma_{1} \dot{z}[n]+F_{v} \dot{x}[n] .
$$

Using the average bristle deflection equations, Eqs. (3.24) and (3.25), it is possible to find the difference equation relationship between $\dot{x}$ and $z$ :

$$
z[n]=\frac{T_{s}}{2}\left[\dot{x}[n-1]+\dot{x}[n-2]+\left(\frac{2}{T_{s}}-\frac{|\dot{x}[n-1]|}{g(\dot{x}[n-1])}\right) z[n-1]+\frac{|\dot{x}[n-2]|}{g(\dot{x}[n-2])} z[n-2]\right] .
$$


Considering that the the generalized derivative of the absolute function at the discontinuous point is zero (it lies in the convex hull $[-1,1]$, however the imprecision is considered tolerable in this work), the first order partial derivative of $z[n]$ with respect to the input variables are:

$$
\begin{aligned}
\frac{\partial z[n]}{\partial \dot{x}[n-1]} & =\frac{T_{s}}{2}\left[1+\left(\frac{|\dot{x}[n-1]|}{g([n-1])^{2}} \frac{\partial g(\dot{x}[n-1])}{\partial \dot{x}[n-1]}-\frac{\operatorname{sgn}(\dot{x}[n-1])}{g(\dot{x}[n-1])}\right) z[n-1]\right] \\
\frac{\partial z[n]}{\partial \dot{x}[n-2]} & =\frac{T_{s}}{2}\left[1+\left(\frac{|\dot{x}[n-2]|}{g([n-2])^{2}} \frac{\partial g(\dot{x}[n-2])}{\partial \dot{x}[n-2]}-\frac{\operatorname{sgn}(\dot{x}[n-2])}{g(\dot{x}[n-2])}\right) z[n-2]\right] \\
\frac{\partial z[n]}{\partial z[n-1]} & =1-\frac{|\dot{x}[n-1]|}{g([n-1])} \frac{T_{s}}{2} \\
\frac{\partial z[n]}{\partial z[n-2]} & =-\frac{|\dot{x}[n-2]|}{g([n-2])} \frac{T_{s}}{2}
\end{aligned}
$$

with:

$$
\frac{\partial g(\dot{x}[n])}{\partial \dot{x}[n]}=-2 \frac{F_{s}-F_{c}}{\sigma_{0}} \exp \left(-\left(\dot{x}[n] / v_{s}\right)^{2}\right) \frac{\dot{x}[n]}{v_{s}{ }^{2}} .
$$

The linearized difference equation that maps the stem velocity into an average bristle deflection can be written as:

$$
\delta z[n]=A_{L L 1} \delta \dot{x}[n-1]+A_{L L 2} \delta \dot{x}[n-2]+A_{L L 3} \delta z[n-1]+A_{L L 4} \delta z[n-2]
$$

where:

$$
\begin{array}{ll}
A_{L L 1}=\left.\frac{\partial z[n]}{\partial \dot{x}[n-1]}\right|_{\substack{\dot{x}[n-1]=\dot{x}_{L 1} \\
z[n-1]=z_{L 1}}} & A_{L L 2}=\left.\frac{\partial z[n]}{\partial \dot{x}[n-2]}\right|_{\substack{\dot{x}[n-2]=\dot{x}_{L 2} \\
z[n-2]=z_{L 2}}}, A_{L L 4}=\left.\frac{\partial z[n]}{\partial z[n-2]}\right|_{\dot{x}[n-2]=\dot{x}_{L 2}}, \\
A_{L L 3}=\left.\frac{\partial z[n]}{\partial z[n-1]}\right|_{\dot{x}[n-1]=\dot{x}_{L 1}} & ,
\end{array}
$$

and $\delta$ denotes the linearization point incremental variable, e.g. $\delta z[n-1]=z[n-1]-z_{L 1}$. Therefore, the average bristle deflection can be rewritten in a transfer function form:

$$
\delta z[n]=G_{z L}\left(q, \dot{x}_{L 1}, \dot{x}_{L 2}, z_{L 1}, z_{L 2}\right) \delta \dot{x}[n] .
$$

The friction force, Eq. (5.16), can be written as a function of $\delta \dot{x}[n]$ and the linearization point (which is omitted in the transfer function for simplicity):

$$
\delta F_{f_{L}}[n]=\left(F_{v}+\sigma_{0} G_{z L}(q)+\sigma_{1} G_{z L}(q) \frac{2}{T_{s}} \frac{1-q^{-1}}{1+q^{-1}}\right) \frac{2}{T_{s}} \frac{1-q^{-1}}{1+q^{-1}} \delta x[n] .
$$

Then, the valve model, Eq. (5.4), is written as:

$$
\begin{aligned}
4 \delta x[n]+\left(\frac{T_{s}^{2} k}{m}-8\right) \delta x[n-1]+ & \left(4+\frac{2 T_{s}^{2} k}{m}\right) \delta x[n-2]+\frac{T_{s}^{2} k}{m} \delta x[n-3]= \\
& \frac{T_{s}^{2}}{m}\left(q^{-1}+2 q^{-2}+q^{-3}\right)\left(S_{a} \delta P[n]-\delta F_{f L}[n]\right) .
\end{aligned}
$$


Eq. (5.24) can be reduced to a transfer function form (omitting the linearization point terms), which tends to be overall stable, as the valve is obviously designed to be bounded input, bounded output stable:

$$
\delta x[n]=G_{L L}(q) \delta P[n] .
$$

\subsubsection{GMS Friction Model}

Given Eq. (3.12), the GMS model friction force is:

$$
F_{f_{G}}[n]=\sum_{i=1}^{N_{M e}} F_{i}[n]+F_{v} \dot{x}[n],
$$

where:

$$
F_{i}[n]=\kappa_{i} \psi_{i}[n]+\nu_{i} \dot{\psi}_{i}[n]
$$

If all Maxwell elements are in presliding state, using the GMS model definition in Eq. (3.26), then Eq. (5.4) is rewritten as:

$$
\begin{aligned}
4 x[n]+\left(\frac{T_{s}^{2} k}{m}-8\right) x[n-1] & +\left(4+\frac{2 T_{s}^{2} k}{m}\right) x[n-2]+\frac{T_{s}^{2} k}{m} x[n-3]= \\
& \frac{T_{s}^{2}}{m}\left(q^{-1}+2 q^{-2}+q^{-3}\right)\left(S_{a} P[n]-F_{f_{G p}}[n]-F_{\text {init }}\right),
\end{aligned}
$$

with:

$$
F_{f_{G p}}[n]=\sum_{i=1}^{N_{M e}} \kappa_{i} x[n]+\nu_{i} x[n] \frac{2}{T_{s}} \frac{1-q^{-1}}{1+q^{-1}} .
$$

Eq. (5.28) can be reduced to a transfer function form:

$$
\delta x[n]=G_{L G p}(q) \delta P[n],
$$

that is defined used incremental variables only to guarantee the notation consistency with the following analysis, since Eq. (5.28) is already linear.

With regard to the sliding state, using Eq. (3.26), the Maxwell elements virtual position are governed by the following difference equation:

$$
\frac{2}{T_{s}} \frac{1-q^{-1}}{1+q^{-1}} \psi_{i}[n+1]=\frac{C \alpha_{i}}{\kappa_{i}}\left(\operatorname{sgn}(\dot{x}[n])-\frac{\kappa_{i} \psi[n]+\nu_{i} \psi_{i}[n] \frac{2}{T_{s}} \frac{1-q^{-1}}{1+q^{-1}}}{\alpha_{i} s(\dot{x}[n])}\right) .
$$

Rearranging the terms of Eq. (5.31), one obtains:

$$
\begin{aligned}
\psi_{i}[n+1]=\frac{T_{s}}{2} \frac{C \alpha_{i}}{\kappa_{i}} \operatorname{sgn}(\dot{x}[n]) & +\frac{T_{s}}{2} \frac{C \alpha_{i}}{\kappa_{i}} \operatorname{sgn}(\dot{x}[n-1])+ \\
& \left(1-\frac{T_{s}}{2} \frac{C}{s(\dot{x}[n])}-\frac{C \nu_{i}}{\kappa_{i} s(\dot{x}[n])}\right) \psi_{i}[n]-\frac{C \nu_{i}}{\kappa_{i} s(\dot{x}[n-1])} \psi_{i}[n-1] .
\end{aligned}
$$


By applying the generalized derivative theorem, the first order partial derivatives of $\psi_{i}$ with respect to the input variables are:

$$
\begin{aligned}
\frac{\partial \psi_{i}[n]}{\partial \psi_{i}[n-1]} & =1-\frac{T_{s}}{2} \frac{C}{s(\dot{x}[n-1])}-\frac{C \nu_{i}}{\kappa_{i} s(\dot{x}[n-1])} \\
\frac{\partial \psi_{i}[n]}{\partial \psi_{i}[n-2]} & =-\frac{C \nu_{i}}{\kappa_{i} s(\dot{x}[n-2])} \\
\frac{\partial \psi_{i}[n]}{\partial \dot{x}[n-1]} & =\left(\frac{T_{s}}{2} \frac{C}{s(\dot{x}[n-1])^{2}} \frac{\partial s(\dot{x}[n-1])}{\partial \dot{x}[n-1]}+\frac{C \nu_{i}}{\kappa_{i} s(\dot{x}[n-1])^{2}} \frac{\partial s(\dot{x}[n-1])}{\partial \dot{x}[n-1]}\right) \psi_{i}[n-1] \\
\frac{\partial \psi_{i}[n]}{\partial \dot{x}[n-2]} & =\frac{C \nu_{i}}{\kappa_{i} s(\dot{x}[n-2])^{2}} \frac{\partial s(\dot{x}[n-2])}{\partial \dot{x}[n-2]} \psi_{i}[n-2] .
\end{aligned}
$$

Thus, the linearized difference equation that maps the stem velocity into a Maxwell element $i$ displacement is:

$$
\delta \psi_{i}[n]=A_{L G 1} \delta \dot{x}[n-1]+A_{L G 2} \delta \dot{x}[n-2]+A_{L G 3} \delta \psi_{i}[n-1]+A_{L G 4} \delta \psi_{i}[n-2],
$$

where:

$$
\begin{aligned}
& A_{L G 1}=\left.\frac{\partial \psi_{i}[n]}{\partial \dot{x}[n-1]}\right|_{\begin{array}{c}
\dot{x}[n-1]=\dot{x}_{L 1} \\
\psi_{i}[n-1]=\psi_{i L 1}
\end{array}} \quad A_{L G 2}=\left.\frac{\partial \psi_{i}[n]}{\partial \dot{x}[n-2]}\right|_{\begin{array}{c}
\dot{x}[n-2]=\dot{x}_{L 2} \\
\psi_{i}[n-2]=\psi_{i L 2}
\end{array}} \\
& A_{L G 3}=\left.\frac{\partial \psi_{i}[n]}{\partial \psi_{i}[n-1]}\right|_{\dot{x}[n-1]=\dot{x}_{L 1}} \quad A_{L G 4}=\left.\frac{\partial \psi_{i}[n]}{\partial z[n-2]}\right|_{\dot{x}[n-2]=\dot{x}_{L 2}} .
\end{aligned}
$$

As in the LuGre model case, the Maxwell elements displacement in sliding state is a nonlinear filter of stem velocity, with variable gain and poles, depending on the previous stem velocities and Maxwell elements displacement. Therefore, it is possible to define a linearized transfer function to calculate the Maxwell element displacement, given the stem velocity:

$$
\delta \psi[n]=G_{\psi_{i} G}\left(q, \dot{x}_{L 1}, \dot{x}_{L 2}, \psi_{i L 1}, \psi_{i L 2}\right) \delta \dot{x}[n] .
$$

With Eqs. (5.36) and (5.27), the linearized valve model with GMS friction model in sliding state is:

$$
\begin{aligned}
4 \delta x[n]+\left(\frac{T_{s}^{2} k}{m}-8\right) \delta x[n-1]+ & \left(4+\frac{2 T_{s}^{2} k}{m}\right) \delta x[n-2]+\frac{T_{s}^{2} k}{m} \delta x[n-3]= \\
& \frac{T_{s}^{2}}{m}\left(q^{-1}+2 q^{-2}+q^{-3}\right)\left(S_{a} \delta P[n]-\delta F_{f_{G s}}[n]\right),
\end{aligned}
$$

with:

$$
\delta F_{f_{G s}}[n]=\sum_{i=1}^{N_{M e}}\left(\kappa_{i}+\nu_{i} \frac{2}{T_{s}} \frac{1-q^{-1}}{1+q^{-1}}\right) \frac{2}{T_{s}} \frac{1-q^{-1}}{1+q^{-1}} G_{\psi_{i} G}(q) \delta x[n] .
$$

Note that the linearization point of $G_{\psi_{i} G}(q)$ is omitted for simplicity. Eq. (5.37) can be rearranged into a transfer function form:

$$
\delta x[n]=G_{L G s}(q) \delta P[n]
$$




\subsubsection{Kano Valve Model}

This model is rather simple, as there is no dynamics, as seen in Algorithm 3.1. If the valve is in the presliding state, then:

$$
x[n]=x[n-1] .
$$

If the valve is in the sliding state, then the stem position is the scaled valve input, depending on previous values of $x$ (the movement direction):

$$
x[n]=G_{K}(q) P[n-1] .
$$

\subsubsection{A Generalized Valve Model}

From the previous sections it can be noted that the valve model maps the diaphragm pressure into stem position using nonlinear filters. It may be assumed that the valve model can be approximated by allowing time variations of the difference equation coefficients, reflecting a linearized valve model, with updated linearization point at each time step:

$$
\left\{\begin{array}{l}
\zeta[n+1]=\Theta[n] \zeta[n]+\Gamma[n] P[n] \\
x[n]=\Omega \zeta[n]
\end{array}\right.
$$

where $\zeta[n] \in \mathbb{R}^{d_{\zeta} \times 1}$ is the internal state vector whose dimension depends on the valve model, $P[n] \in \mathbb{R}$ is the diaphragm pressure, $\Theta[n] \in \mathbb{R}^{d_{\zeta} \times d_{\zeta}}, \Gamma[n] \in \mathbb{R}$ are bounded operator sequences (e.g. $\sup \{\|\Theta[n]\|<\infty \mid n \in \mathbb{N}\})$ and $\Omega \in \mathbb{R}^{1 \times d_{\zeta}}$.

Solving Eq. (5.42), one obtains (HALANAY; IONESCU, 1994):

$$
x[n]=\Omega S_{m}(n-1,0) \zeta[0]+\sum_{i=1}^{n} \Omega S_{m}(n-i, 0) \Gamma[n-i] P[n-i],
$$

where $S_{m}: \mathbb{R}^{d_{\zeta} \times 1} \mapsto \mathbb{R}^{d_{\zeta} \times 1}$ is defined as:

$$
S_{m}(i, j)=\left\{\begin{array}{l}
I, \quad \text { if } i=j \\
\Theta[i] \Theta[i-1] \ldots \Theta[j], \quad \text { if } i>j \\
0, \quad \text { if } i<j,
\end{array}\right.
$$

here $I$ is the identity matrix. Therefore, from Eq. (5.43), the stem position can be approximated by a time varying function that depends on the friction parameter vector:

$$
x[n]=T\left(n, \theta_{f}, P[n]\right) .
$$

\subsubsection{Open Loop Parameter Estimation Analysis}

Even though estimating the parameters of an open loop system usually does not lead to estimation bias, its stochastic analysis is carried out as an introduction for the 
closed loop systems analysis. In Chapter 4 the measured variables are considered perfectly known, which is an assumption that does not hold in practical cases. Frequently, the measured variables are considered corrupted by measurement noise that is usually modeled as an additive white noise with zero mean and a given variance:

$$
\begin{array}{rlrl}
x_{m}[n] & =x[n]+w_{x}[n], & & w_{x} \sim \mathcal{N}\left(0,{\sigma_{w_{x}}}^{2}\right) \\
P_{m}[n]=P[n]+w_{P}[n], & & w_{P} \sim \mathcal{N}\left(0,{\sigma_{w_{P}}}^{2}\right) .
\end{array}
$$

The random variables $w_{x}$ and $w_{P}$ are considered independent, as the measurement systems are not coupled in any way. Using Eq. (5.46), the mean squared prediction error, defined in Eqs. (4.2) and (4.3), can be updated (omitting some parameters for simplification):

$$
V_{n_{f}}\left(\hat{\theta}_{f}\right)=\frac{1}{n_{f}} \sum_{n=1}^{n_{f}}\left(\hat{x}\left(n, \hat{\theta}_{f}\right)-x_{m}[n]\right)^{2}=\frac{1}{n_{f}} \sum_{n=1}^{n_{f}} e_{x_{m}}[n]^{2} .
$$

Assuming that the plant and model have the same structure, the residual, $e_{x_{m}}[n]$, is calculated as:

$$
e_{x_{m}}[n]=T\left(n, \hat{\theta}_{f}, P[n]+w_{P}[n]\right)-\left(T\left(n, \theta_{f}, P[n]\right)+w_{x}[n]\right) .
$$

Expanding the squared residual, one obtains:

$$
e_{x_{m}}[n]^{2}=\Delta T[n]^{2}-2 \Delta T[n]+w_{x}[n]^{2}
$$

with:

$$
\Delta T[n]=T\left(n, \hat{\theta}_{f}, P[n]+w_{P}[n]\right)-T\left(n, \theta_{f}, P[n]\right)
$$

Let $E$ denote the expectation and averaging operator (LJUNG, 1999), then:

$$
V_{n_{f}}\left(\hat{\theta}_{f}\right)=E\left(\Delta T[n]^{2}\right)-2 E\left(\Delta T[n] w_{x}\right)+E\left(w_{x}^{2}\right) .
$$

By using the $T$ definition, from Eq. (5.43), the first term of Eq. (5.51) can be expanded:

$$
E\left(\Delta T[n]^{2}\right)=E\left((\Delta x[n])^{2}\right)+2 E\left(\Delta x[n] G_{S}[n] w_{P}[n]\right)+E\left(\left(G_{S}[n] w_{P}[n]\right)^{2}\right),
$$

with:

$$
\begin{array}{r}
\Delta x[n]=\hat{\Omega} \hat{S}(n-1,0) \hat{\zeta}[0]+\sum_{i=1}^{n} \hat{\Omega} \hat{S}(n-i, 0) \hat{\Gamma}[n-i] P[n-i]- \\
\quad \Omega S(n-1,0) \zeta[0]-\sum_{i=1}^{n} \Omega S(n-i, 0) \Gamma[i] P[n-i]
\end{array}
$$

and

$$
G_{S}[n] w_{P}[n]=\sum_{i=1}^{n} \hat{\Omega} \hat{S}(n-i, 0) \hat{\Gamma}[n-i] w_{P}[n-i]
$$


From Eq. (5.54), one may note that $E\left(G_{S}[n] w_{P}[n]\right)=E\left(w_{P}[n]\right)=0$, as the term $G_{S}[n]$ acts as a linear filter of the random variable (assuming the stability of $\hat{S}$ ). The variance, however, is affected by $G_{S}[n]$ transformation. If the state-transition operator, $\hat{S}$, is stable, then it follows that $E\left(\Delta x[n] G_{S}[n] w_{P}[n]\right)=0$. It is reasonable to assume that the statetransition operator is stable, since the valves are designed to be stable and an unstable $\hat{S}$ would readily be discarded by the estimator, due to its high mean squared prediction error. Yet, perfect valve and friction parameters estimation lead to $E\left(\Delta x[n] G_{S}[n] w_{P}[n]\right)=0$, as well. The second term can be expanded as well:

$$
\begin{array}{r}
E\left(\Delta T[n] w_{x}[n]\right)=E\left(\left[\hat{\Omega} \hat{S}(n-1,0) \hat{\zeta}[0]+\sum_{i=1}^{n} \hat{\Omega} \hat{S}(n-i, 0) \hat{\Gamma}[n-i]\left(P[n-i]+w_{P}[n]\right)-\right.\right. \\
\left.\left.\quad \Omega S(n-1,0) \zeta[0]-\sum_{i=1}^{n} \Omega S(n-i, 0) \Gamma[n-i] P[n-i]\right] w_{x}\right) .
\end{array}
$$

By noting that the random variables have zero mean and are independent, then Eq. (5.55) reduces to:

$$
E\left(\Delta T[n] w_{x}[n]\right)=\hat{\Omega} \hat{S}(n, 0) \hat{\zeta}[0]-\Omega S(n, 0) \zeta[0] .
$$

The term $E\left(\Delta T[n] w_{x}\right)=0$, given the conditions already discussed (state-transition operators stability and a perfect estimation of valve and friction parameters).

From the previous analysis, Eq. (5.51) is reduced to:

$$
V_{n_{f}}\left(\hat{\theta}_{f}\right)=E\left(\Delta x[n]^{2}\right)+{\sigma_{w_{x}}}^{2}+E\left(G_{S}[n] w_{P}[n]^{2}\right) .
$$

Eq. (5.57) shows that when $\hat{\theta}_{f}=\theta_{f}, V_{n_{f}}\left(\hat{\theta}_{f}\right)$ is at its minimal value, which means that the open loop estimation method is unbiased, even in presence of measurement noise.

\subsubsection{Closed Loop Parameter Estimation Analysis}

From Figure 5.1, it is possible to calculate the functions that map deviations from the desired set point to the position controller output. The OP can be calculated from SP and $x_{m}$ :

$$
\mathrm{OP}[n]=C_{p}\left(q, x_{m}[n-1], \mathrm{SP}[n-1]\right)
$$

A generic function can be used to map OP into $P$ :

$$
P[n]=f_{\mathrm{I} / \mathrm{P}}\left(\mathrm{OP}[n], \theta_{\mathrm{I} / \mathrm{P}}, q\right) .
$$

The analysis is essentially the same as in Subsection 5.3.6, however the diaphragm pressure is now correlated to the stem position measurement noise.

The difference from the open loop case lies on the terms $E\left(\Delta T[n] w_{x}[n]\right)$ and $E\left(\Delta x[n] G_{S}[n] w_{P}[n]\right)$, which are not zero, because $E\left(P[n] w_{x}[n]\right) \neq 0$. The state-transition 
operator acts as a filter and may even amplify the correlation between these variables. In closed loop, the resulting mean squared prediction error is:

$$
\begin{aligned}
V_{n_{f}}\left(\hat{\theta}_{f}\right)=E\left(\Delta x[n]^{2}\right)-2 E\left(\Delta T[n] w_{x}[n]\right)+2 E\left(\Delta x[n] G_{S}[n] w_{P}[n]\right)+ \\
\sigma_{w_{x}}{ }^{2}+E\left[\left(G_{S}[n] w_{P}[n]\right)^{2}\right] .
\end{aligned}
$$

From Eq. (5.60) it is possible to conclude that in the closed loop case, the parameter estimation is biased, since when $\hat{\theta}_{f}=\theta_{f}, V_{n_{f}}\left(\hat{\theta}_{f}\right)$ is not at its minimal value, as shown by Hagenblad, Ljung and Wills (2008).

A solution for this problem is to lower the correlation between $P$ and $w_{x}$, by using a position controller with low gain and no integrator during the parameter estimation experiment. By doing so, the term $2 E\left(\Delta T[n] w_{x}[n]\right)$ tends to be minimized, improving the estimation performance.

Assume that the stem position and SP are normalized to $0-100 \%$. Then, if $C_{p}=0.1$ during the excitation period, a $10 \%$ correction is applied in OP when the stem position error is $100 \%$, which is not acceptable. To ensure that the overall valve performance is fair, the SP may be modified to:

$$
\mathrm{SP}[n]=20\left(\mathrm{SP}_{H}[n]-50\right)
$$

where $\mathrm{SP}_{H}$ is the output from the hydraulic system controller, $C_{H}$. Therefore, $\mathrm{SP} \in$ $[-1000,1000]$ and OP $\in[-100,100]$. Note that OP has to compensate the exc signal, the reason why its lower range is -100 .

\subsection{Simulated Tests}

In this test, $C_{p}$ is a discrete $\mathrm{P}$ controller with $10^{-3} \mathrm{~s}$ sampling time and proportional gain, $K_{p}=0.1$. The hydraulic system is considered the same as presented by Hidalgo and Garcia (2017), which corresponds to a Wiener system with a linear dynamic system:

$$
\frac{x_{w}(s)}{x(s)}=\frac{1}{3.375 s+1}
$$

and nonlinear static function:

$$
Q\left(x_{w}(t)\right)=-100442.87 x_{w}(t)^{2}-630.57 x_{w}(t)+101.54
$$

Note that the flow rate $Q$ is normalized. The hydraulic system controller $\left(C_{H}\right)$ is a parallel digital PI controller with $10^{-3}$ s sampling time, proportional gain $K_{H}=0.675$ and $K_{i H}=0.2 \mathrm{rep} / \mathrm{s}$, which is calculated to result in a closed loop time constant $50 \%$ higher than the process time constant, which represents a system with low natural excitation. The SP (output of $C_{H}$ ) is modified as presented in Section 5.3. 
The valve stem position and diaphragm pressure measurements are considered corrupted by white noise, resulting in $25 \mathrm{~dB}$ SNR. The valve flow rate set point, $\mathrm{SP}_{Q}$, is considered a white noise signal with $60 \%$ mean, $0.25 \%$ standard deviation and a sampling time of $120 \mathrm{~s}$, which represents a valve that does not exhibit rich information during normal operation (the worst case). The valve and friction parameters (plant) are the ones shown in Tables 3.1 and 3.2.

The valve deadband is estimated as presented in Algorithm 5.1 and the I/P model parameters are estimated as suggested in Section 5.2, using the valve with Karnopp friction model simulated data as the plant. The signals used for the estimation are presented in Figures 5.6 and 5.7, and the results are shown in Table 5.2. It is possible to verify that the $\mathrm{I} / \mathrm{P}$ model parameter estimation performance is excellent. Despite the fact that the valve deadband estimation is much less precise, it is still tolerable, since the search space admits an error of $30 \%$ in this estimation.

Table 5.2 - Closed loop I/P and valve signature curve parameters estimation in the simulated case.

\begin{tabular}{lr} 
Parameter & Est. Error (\%) \\
\hline$S_{0}$ & 4.84 \\
$\hat{P}_{\min }$ & -0.12 \\
$\hat{K}_{P_{g}}$ & -0.02 \\
$\hat{\tau}_{\mathrm{I} / \mathrm{P}}$ & 0.02 \\
\hline
\end{tabular}

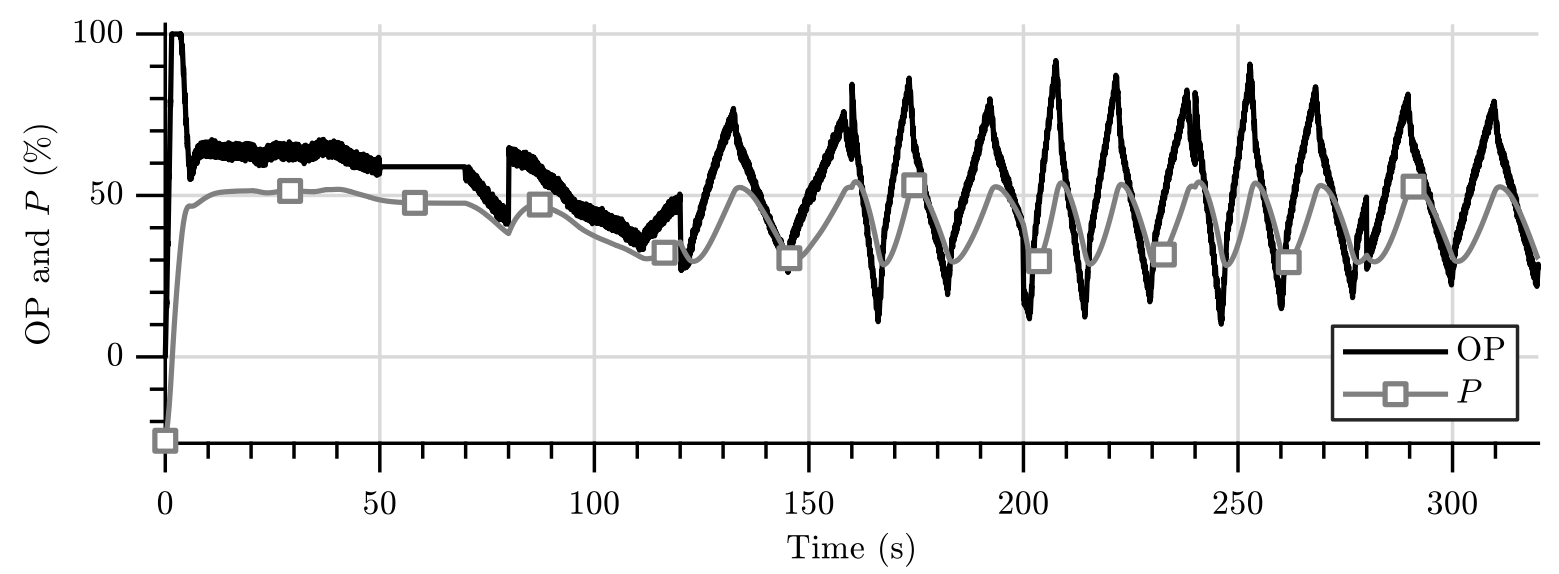

Figure 5.6 - Signals used to estimate the I/P model parameters and $S_{0}$ in the simulated closed loop case.

Source: from Author. 


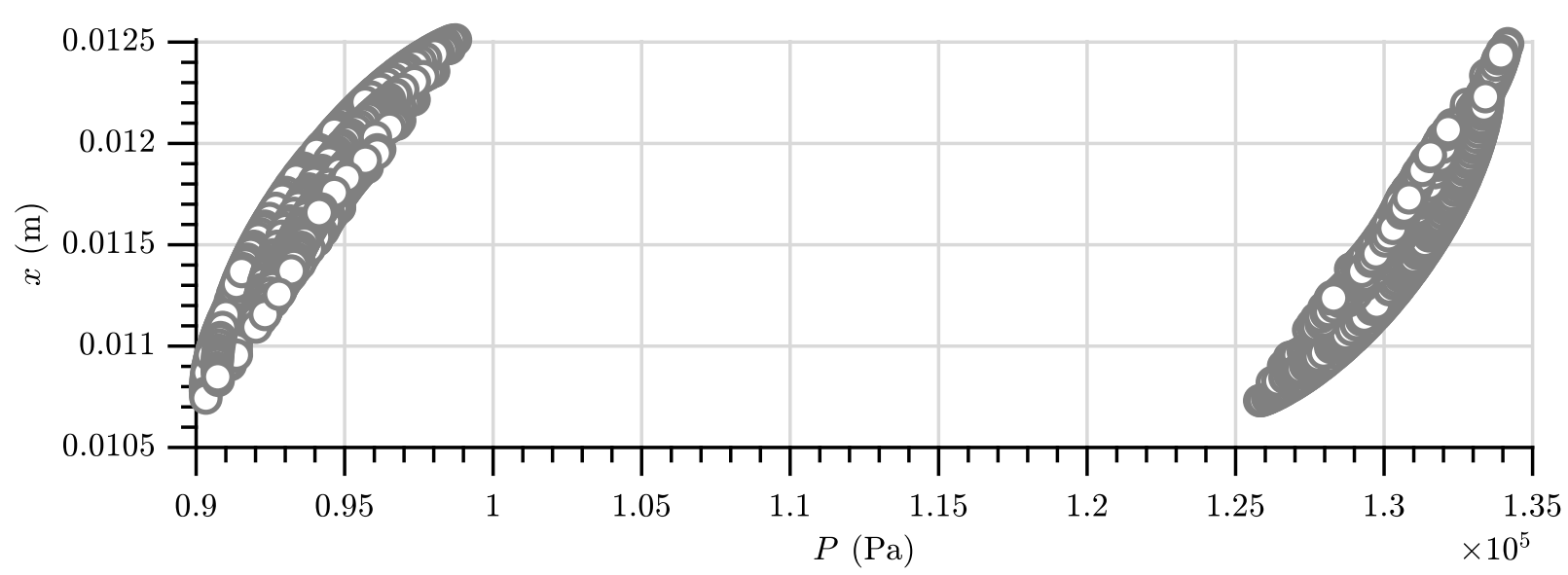

Figure 5.7 - Valve signature curve with only nonzero stem velocities used to estimate $S_{0}$ in the simulated closed loop case.

Source: from Author.

The signals used to estimate $k, F_{\text {init }}$ and $\theta_{f}$ are shown in Figure 5.8. Note that the valve stem position does not deviate substantially.

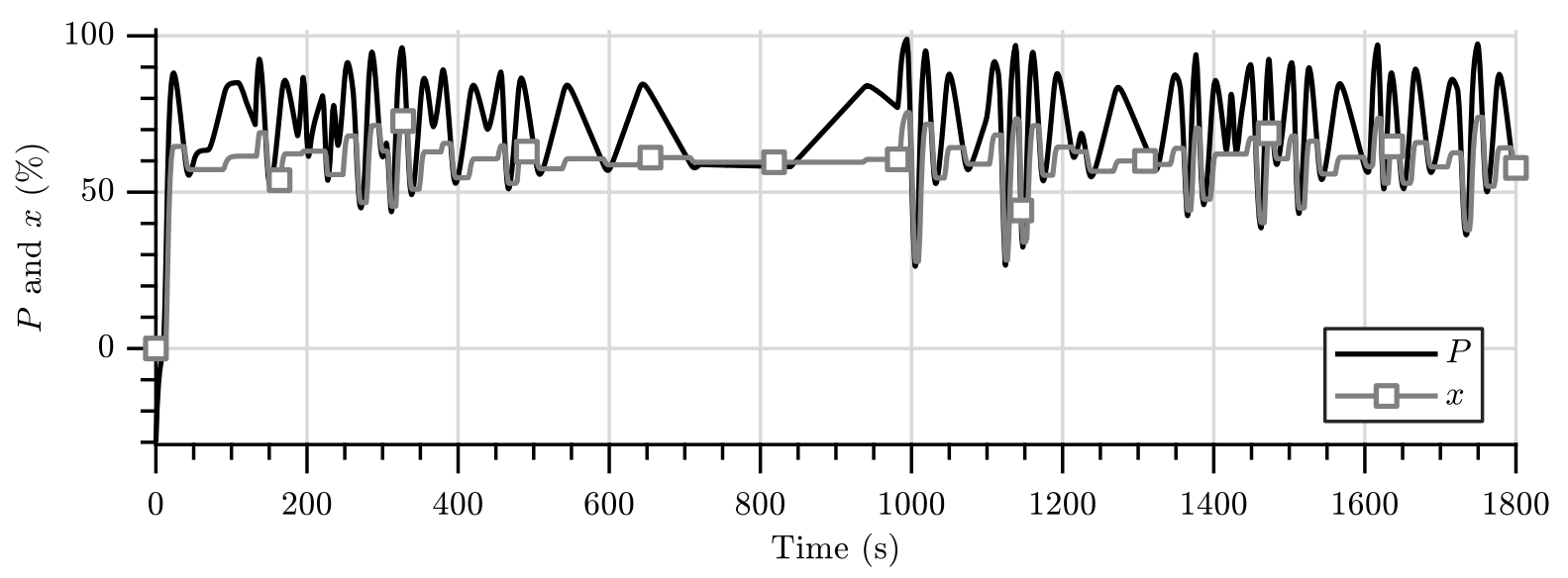

Figure 5.8 - Signals used to estimate $k, F_{\text {init }}$ and $\theta_{f}$ in the simulated closed loop case.

Source: from Author.

To estimate $k$ and $F_{\text {init }}$, it is assumed that the parameters have deviated $10 \%$ from the original value and the friction parameters are perfectly known. The results are satisfactory, as can be seen in Table 5.3.

Table 5.3 - Friction parameters estimation test with simulated data results.

\begin{tabular}{lrr} 
Model & \multicolumn{2}{c}{ Est. error $(\%)$} \\
& $\hat{k}$ & $\hat{F}_{\text {init }}$ \\
\hline Karnopp & -0.029 & 0.026 \\
LuGre & -0.219 & 0.156 \\
GMS & -0.139 & 0.132 \\
\hline
\end{tabular}

Source: from Author. 
Finally, to estimate $\theta_{f}$, the $k$ and $F_{\text {init }}$ parameters are considered known. The estimation results are presented in Tables 5.4 to 5.8. The best performance is provided by the GMS model, even though the parameter estimation errors were high, which may indicate that the model is over-parameterized, since even the Karnopp model, with almost perfect estimates, is not able to reach the same efficiency. As for the LuGre model, the performance is fair and the estimation error is similar to the open loop simulated case. Finally, the Kano model has the lower prediction efficiency and presented high parameter errors, specially regarding $J$ parameter, which is a pattern already observed, with less severity, in the open loop simulated case.

Table 5.4 - Closed loop friction parameter estimation test results with simulated data.

\begin{tabular}{lrrr} 
Model & fit $(\%)$ & Run Time $(\mathrm{h})$ & Func. Evals. \\
\hline Kano & 98.19 & 0.43 & 7885 \\
Karnopp & 99.10 & 42.7 & 21922 \\
LuGre & 98.55 & 217.9 & 34887 \\
GMS & 99.49 & 312.7 & 97614
\end{tabular}

Func. Evals.: refers to the number of objective function evaluations.

Source: from Author.

Table 5.5 - Closed loop Kano parameter estimation error in a test with simulated data.

$$
\begin{array}{ccccc}
\hat{S}(\%) & \hat{J}(\%) & \hat{D}(\%) & \hat{x}_{\min P}(\%) & \hat{x}_{\max P}(\%) \\
\hline-4.41 & -98.12 & -6.31 & -0.03 & -0.49 \\
\hline \multicolumn{5}{c}{\text { Source: from Author. }}
\end{array}
$$

Table 5.6 - Closed loop Karnopp parameter estimation error in a test with simulated data.

\begin{tabular}{cccc}
$\hat{F}_{c}(\%)$ & $\hat{F}_{s}(\%)$ & $\hat{F}_{v}(\%)$ & $\hat{v}_{s}(\%)$ \\
\hline-0.01 & -0.01 & -0.41 & 0.41 \\
\hline \multicolumn{4}{c}{ Source: from Author. }
\end{tabular}

Table 5.7 - Closed loop LuGre parameter estimation error in a test with simulated data.

$$
\begin{array}{cccccc}
\hat{F}_{c}(\%) & \hat{F}_{s}(\%) & \hat{F}_{v}(\%) & \hat{v}_{s}(\%) & \hat{\sigma}_{0}(\%) & \hat{\sigma}_{1}(\%) \\
\hline-0.32 & -0.16 & 0.64 & 1.73 & 17.60 & -97.5 \\
\hline \multicolumn{6}{c}{\text { Source: from Author. }}
\end{array}
$$


Table 5.8 - Closed loop GMS parameters estimation error, test with simulated data.

\begin{tabular}{ccccccc}
$\hat{F}_{c}(\%)$ & $\hat{F}_{s}(\%)$ & $\hat{F}_{v}(\%)$ & $\hat{v}_{s}(\%)$ & $\hat{\kappa}_{1}(\%)$ & $\hat{\kappa}_{2}(\%)$ & $\hat{\kappa}_{3}(\%)$ \\
\hline-16.83 & -0.26 & 1.58 & $1.55 \cdot 10^{4}$ & -30.16 & 60.83 & 44.99 \\
\hline$\hat{\nu}_{1}(\%)$ & $\hat{\nu}_{2}(\%)$ & $\hat{\nu}_{3}(\%)$ & $\hat{\alpha}_{1}(\%)$ & $\hat{\alpha}_{2}(\%)$ & $\hat{\alpha}_{3}(\%)$ & $\hat{C}(\%)$ \\
\hline $2.00 \cdot 10^{3}$ & $7.61 \cdot 10^{3}$ & 70.96 & -27.53 & 112.48 & -36.92 & -88.17 \\
\hline \multicolumn{7}{c}{ Source: from Author. }
\end{tabular}

\subsection{Experimental Tests}

The experimental data is obtained using the FPP presented in Chapter 2. The valve position controller, $C_{p}$, is a proportional gain with $K_{p}=0.1$, as already discussed in Sections 5.4 and 5.3.

The hydraulic system dynamics is approximated by a first order plus dead time transfer function, estimated with data obtained using rectangular signal excitation, resulting in:

$$
\frac{Q(s)}{x(s)}=\frac{-1.124}{1.815 s+1} \mathrm{e}^{-0.14 s}
$$

The hydraulic system controller, $C_{H}$, is calculated using the Direct Synthesis (DS) method, to result in a closed loop time constant response $50 \%$ slower than the open loop time constant, which is a PI controller with proportional gain $K_{H}=0.564$ and integral gain $K_{i H}=0.31$. The flow rate set point $\mathrm{SP}_{Q}$ is the same shown in Section 5.4.

The I/P model parameters and the valve deadband are estimated using the proposed method shown in Section 5.2 and Algorithm 5.1. The results are summarized in Table 5.9 and the signals used for the estimations are shown in Figures 5.9 to 5.11. Once again, the $S_{0}$ estimation precision is poor, however the search space is wide enough to accommodate such errors (note that the teflon deadband is lower than $2 \%$ and its error is negative). Note the different pattern in deviations from the open loop I/P model parameter estimation, regarding the graphite and teflon gasket valves, as the former presents better estimates for $P_{\min }$ and $K_{P_{g}}$, while the latter shows a better accuracy for $\tau_{\mathrm{I} / \mathrm{P}_{r}}$. This happens because the graphite gasket takes longer to overcome the exc signal, resulting in an excitation signal that is similar to ramps, with only a few high velocity variations, whereas the teflon gasket valve is able to readily compensate exc and therefore the resulting excitation signal is approximately constant with a few spikes. These imprecisions do not influence significantly the friction estimation, as it is used only for designing the signal filters in the closed loop method. As the I/P converter is highly nonlinear, it is suggested that the filters use $\tau_{\mathrm{I} / \mathrm{P}_{s}}$ obtained in the open loop case, regardless of the new closed loop estimates, as the passband is wide enough. Also, the experimental findings indicate that the fit threshold has to be low in order to detect I/P model defects (e.g. lower than 50\%). 
Table 5.9 - Closed loop I/P model and $S_{0}$ parameter estimation deviation from the open loop case.

\begin{tabular}{lrr} 
Parameter & \multicolumn{3}{c}{$\begin{array}{c}\text { Dev. Open Loop (\%) } \\
\text { Graphite }\end{array}$} & Teflon \\
\hline$S_{0}$ & 10.53 & -32.31 \\
$\hat{P}_{\min }$ & 0.34 & -14.64 \\
$\hat{K}_{P_{g}}$ & -2.98 & 9.96 \\
$\hat{\tau}_{\mathrm{I} / \mathrm{P}_{r}}$ & -81.66 & -11.76 \\
\hline \multicolumn{3}{c}{ Source: from Author. }
\end{tabular}

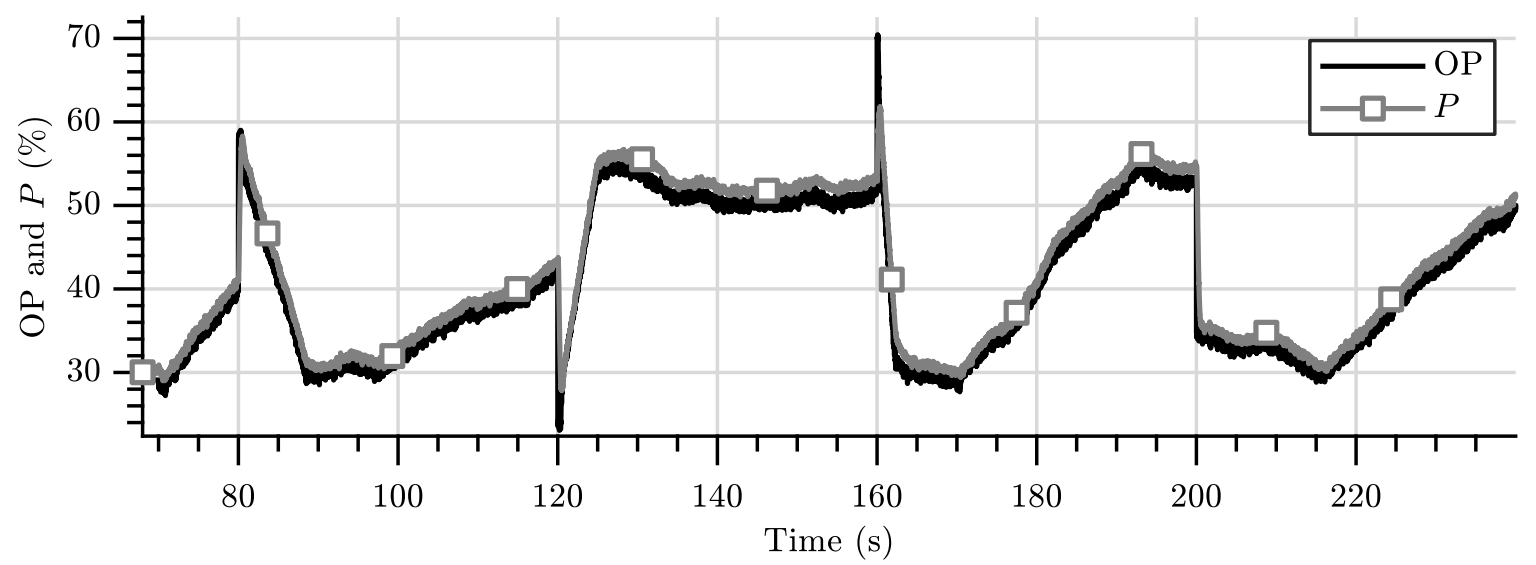

Figure 5.9 - Signals used to estimate the graphite gasket valve I/P model parameters and $S_{0}$.

Source: from Author.

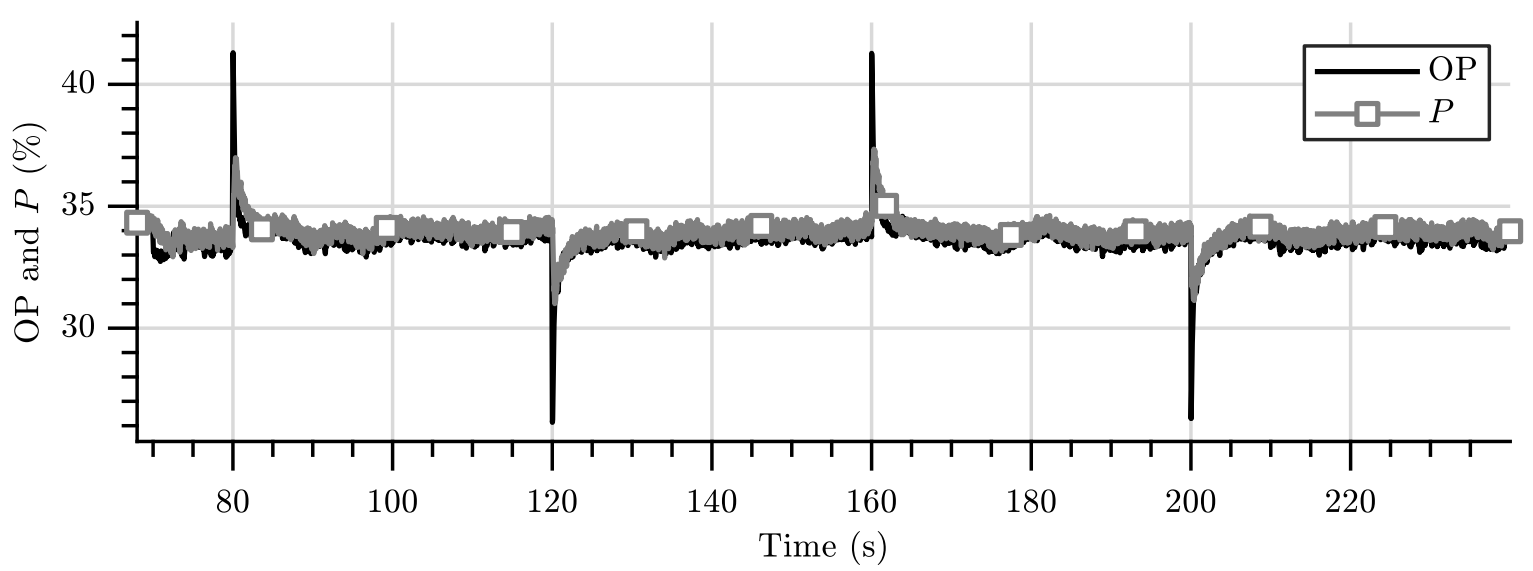

Figure 5.10 - Signals used to estimate the teflon gasket valve I/P model parameters and $S_{0}$.

Source: from Author.

Contrary to the closed loop parameter estimation in the simulated tests, in the experimental case, $k$ and $F_{\text {init }}$ parameters were estimated together with $\theta_{f}$, as this scenario was not tested in the simulated tests.

The estimation performance and comparison to the open loop estimated parameters are presented in Tables 5.10 to 5.15. With respect to the graphite gasket valve, in general, 


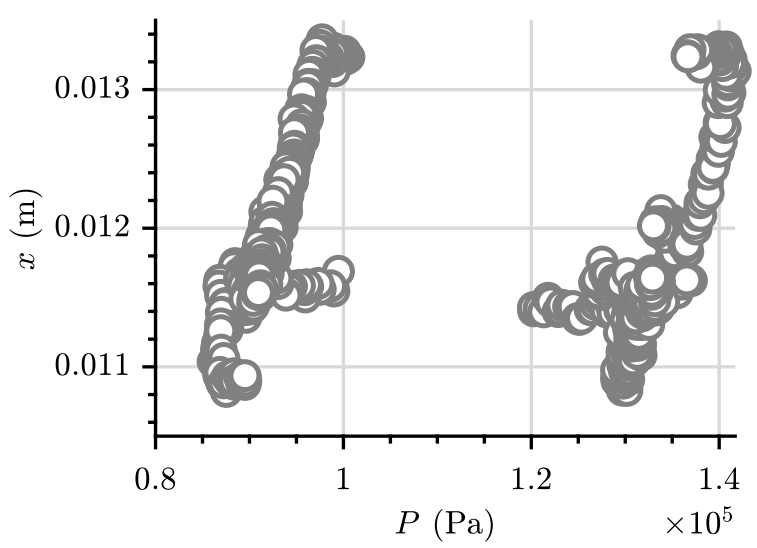

(a) Graphite gasket valve.

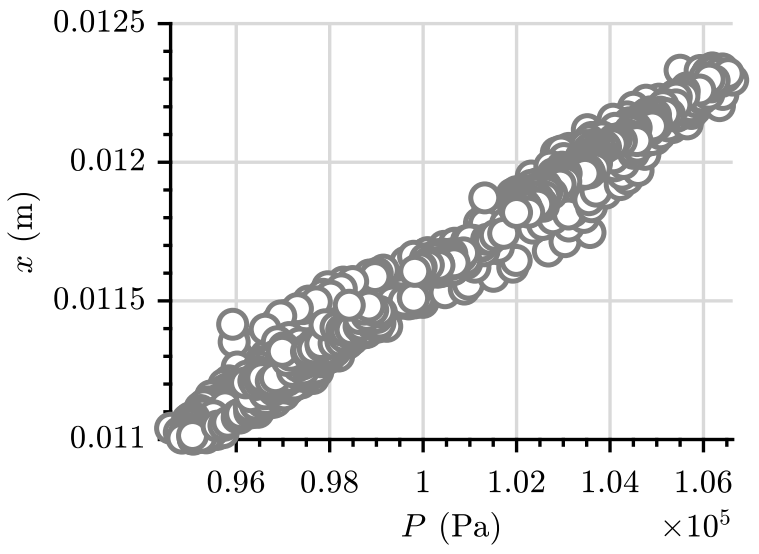

(b) Teflon gasket valve.

Figure 5.11 - Valves signature curves with only nonzero stem velocities used to estimate $S_{0}$.

Source: from Author.

the $f$ it value calculated using the validation dataset decreased roughly $2 \%$ for every model, when compared to the open loop estimated parameters, except for the Kano model, which has a similar $f i t$ index. It is interesting to note that the validation dataset has a $f$ it index higher than the estimation dataset. This characteristic occurred because the fit index penalizes more rigorously the prediction errors that are close to the dataset mean value, which is the case of the estimation dataset, since it presents a low stem position standard deviation, as can be seen in the following subsection. The spring constant and the initial force were pretty consistent when compared to the open loop case. The Karnopp, LuGre and GMS model, presented a consistent decrease in the $F_{s}$ value, whereas $F_{c}$ and $v_{s}$ varied without a visible pattern. It must be pointed out that pretty similar results can be obtained with several different combinations of $F_{c}$ and $v_{s}$, as can be inferred from the Stribeck curve. The $\sigma_{1}$ from LuGre model, and $\kappa_{i}, \nu_{i}$ and $\alpha_{i}$, from GMS model, also presented high deviations, however, these parameters have low influence in the overall valve behavior (as already discussed in Section 4.3). As previously observed in the open loop test, the Kano model presents a relevant underestimation of $J$ parameter, as well as high deviations in $S$ and $D$ parameters. Note that a high $x_{\min \mathrm{P}}$ deviation is not so relevant, as this value is close to zero.

Regarding the teflon gasket valve, the estimation performance in closed loop is excellent. The fit index using the validation dataset is very close to those obtained when the parameters are estimated in open loop, except for the Kano model, in which its fit index increased. This increase is explained by the fact that in closed loop, the maximum and minimum observed stem positions are estimated altogether with the friction parameters, improving the overall estimation performance. The huge closed loop parameter deviations from the open loop estimates are not alarming, as the teflon gasket valve has numerically small friction parameters, that do not affect substantially the overall stem 
position prediction.

Table 5.10 - Closed loop graphite gasket valve model estimation performance.

\begin{tabular}{lrrrr} 
Model & fit (\%) & fit val. (\%) & Run Time (h) & Func. Evals. \\
\hline Kano & 92.18 & 94.29 & 0.02 & 7037 \\
Karnopp & 93.29 & 94.80 & 56.21 & 32728 \\
LuGre & 93.16 & 94.90 & 93.51 & 45878 \\
GMS & 93.48 & 94.89 & 138.43 & 38193
\end{tabular}

fit val.: indicates to the fit index evaluated using the open loop validation dataset and Func. Evals.: refers to the number of objective function evaluations.

Source: from Author.

Table 5.11 - Closed loop teflon gasket valve model estimation performance.

\begin{tabular}{lrrrr} 
Model & fit $(\%)$ & fit val. (\%) & Run Time $(\mathrm{h})$ & Func. Evals. \\
\hline Kano & 98.18 & 98.69 & 0.04 & 15834 \\
Karnopp & 98.95 & 99.39 & 44.81 & 26288 \\
LuGre & 98.95 & 99.39 & 60.96 & 33862 \\
GMS & 98.95 & 99.39 & 161.40 & 49553
\end{tabular}

fit val.: indicates to the fit index evaluated using the validation dataset and Func. Evals.: refers to the number of objective function evaluations.

Source: from Author.

Table 5.12 - Closed loop Kano parameter estimation deviation from open loop tests.

\begin{tabular}{lccccc} 
& $\hat{S}$ & $\hat{J}$ & $\hat{D}$ & $\hat{x}_{\min \mathrm{P}}$ & $\hat{x}_{\max \mathrm{P}}$ \\
\hline Graphite (\%) & -11.23 & -99.89 & -24.3 & -42.86 & -1.11 \\
Teflon (\%) & -96.17 & -97.95 & 100.00 & 3.41 & 1.37 \\
\hline \multicolumn{6}{c}{ Source: from Author. }
\end{tabular}

Table 5.13 - Closed loop Karnopp parameter estimation deviation from open loop tests.

\begin{tabular}{lcccccc} 
& $\hat{k}$ & $\hat{F}_{\text {init }}$ & $\hat{F}_{c}$ & $\hat{F}_{s}$ & $\hat{F}_{v}$ & $\hat{v}_{s}$ \\
\hline Graphite (\%) & 0.17 & -1.91 & -40.89 & -7.2 & -13.56 & 82.34 \\
Teflon (\%) & -0.06 & -49.86 & 100.00 & 100.00 & -83.77 & -98.23 \\
\hline \multicolumn{7}{c}{ Source: from Author. }
\end{tabular}


Table 5.14 - Closed loop LuGre parameter estimation deviation from open loop tests.

\begin{tabular}{|c|c|c|c|c|c|c|}
\hline & $\hat{k}$ & $\hat{F}_{\text {init }}$ & $\hat{F}_{c}$ & $\hat{F}_{s}$ & $\hat{F}_{v}$ & $\hat{v}_{s}$ \\
\hline Graphite (\%) & -0.13 & -1.48 & -24.73 & -6.87 & -10.19 & 27.71 \\
\hline Teflon (\%) & -0.06 & -49.87 & -27.61 & -41.82 & -99.49 & $6.29 \cdot 10^{5}$ \\
\hline & \multicolumn{2}{|c|}{ Model } & $\hat{\sigma}_{0}$ & $\hat{\sigma}_{1}$ & & \\
\hline & \multicolumn{2}{|c|}{ Graphite (\%) } & 5.58 & 147.1 & & \\
\hline & \multicolumn{2}{|c|}{ Teflon (\%) } & -25.50 & $7.02 \cdot 10^{3}$ & & \\
\hline
\end{tabular}

Table 5.15 - Closed loop GMS parameter estimation deviation from open loop tests.

\begin{tabular}{lcccccc} 
Model & $\hat{k}$ & $\hat{F}_{\text {init }}$ & $\hat{F}_{c}$ & $\hat{F}_{s}$ & $\hat{F}_{v}$ & $\hat{v}_{s}$ \\
\hline Graphite (\%) & 0.33 & -2.31 & 4.12 & -7.61 & 41.45 & -26.08 \\
Teflon (\%) & -0.06 & -47.47 & -3.36 & 11.41 & -95.09 & -65.48 \\
\hline
\end{tabular}

\begin{tabular}{lcccccc} 
Model & $\hat{\kappa}_{1}$ & $\hat{\kappa}_{2}$ & $\hat{\kappa}_{3}$ & $\hat{\nu}_{1}$ & $\hat{\nu}_{2}$ & $\hat{\nu}_{3}$ \\
\hline Graphite (\%) & 13.13 & -35.65 & 2.11 & -90.77 & -43.43 & 179.1 \\
Teflon (\%) & $1.81 \cdot 10^{3}$ & -77.36 & -96.03 & $1.01 \cdot 10^{3}$ & 138.91 & 1.25 \\
\hline
\end{tabular}

\begin{tabular}{lcccc} 
Model & $\hat{\alpha}_{1}$ & $\hat{\alpha}_{2}$ & $\hat{\alpha}_{3}$ & $\hat{C}$ \\
\hline Graphite (\%) & -58.15 & 12767.66 & 20959.04 & 0.56 \\
Teflon (\%) & $2.79 \cdot 10^{5}$ & $2.67 \cdot 10^{3}$ & -4.26 & -0.07 \\
\hline \multicolumn{5}{c}{ Source: from Author. }
\end{tabular}

\subsubsection{Graphite Gasket Model Prediction Analysis}

The model predictions, using the closed loop estimated parameters, are compared to the estimation dataset (closed loop) and validation dataset (open loop), as presented in Figures 5.12 to 5.23. Visually, the Kano model has a slight higher residuals and the worst stem velocity prediction accuracy, as it presented stick and slip behavior, where it is not visible in the measured dataset. The other models presented pretty similar efficiency. 

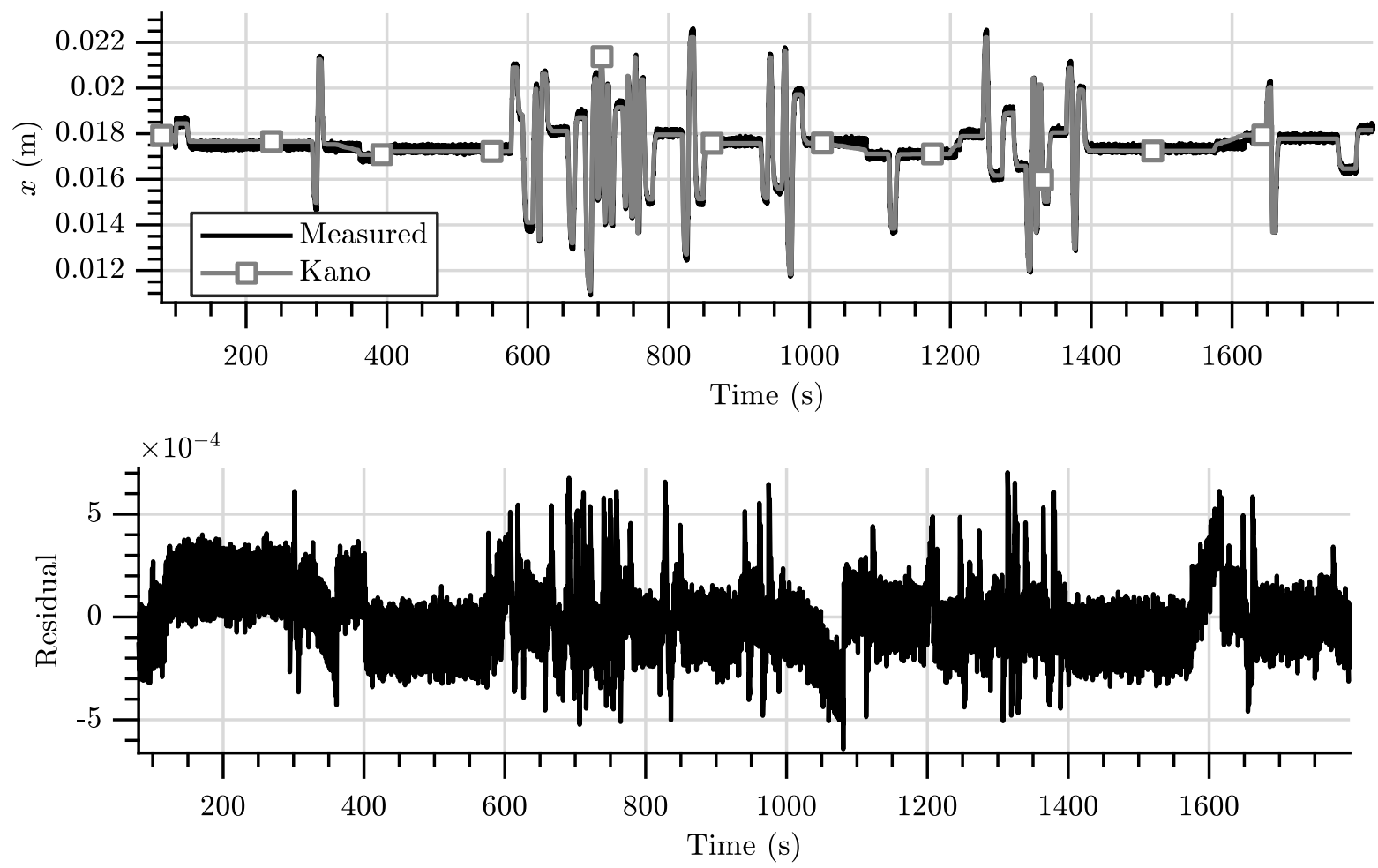

Figure 5.12 - Kano model graphite gasket valve residual with model parameters estimated in closed loop using the estimation dataset.

Source: from Author.
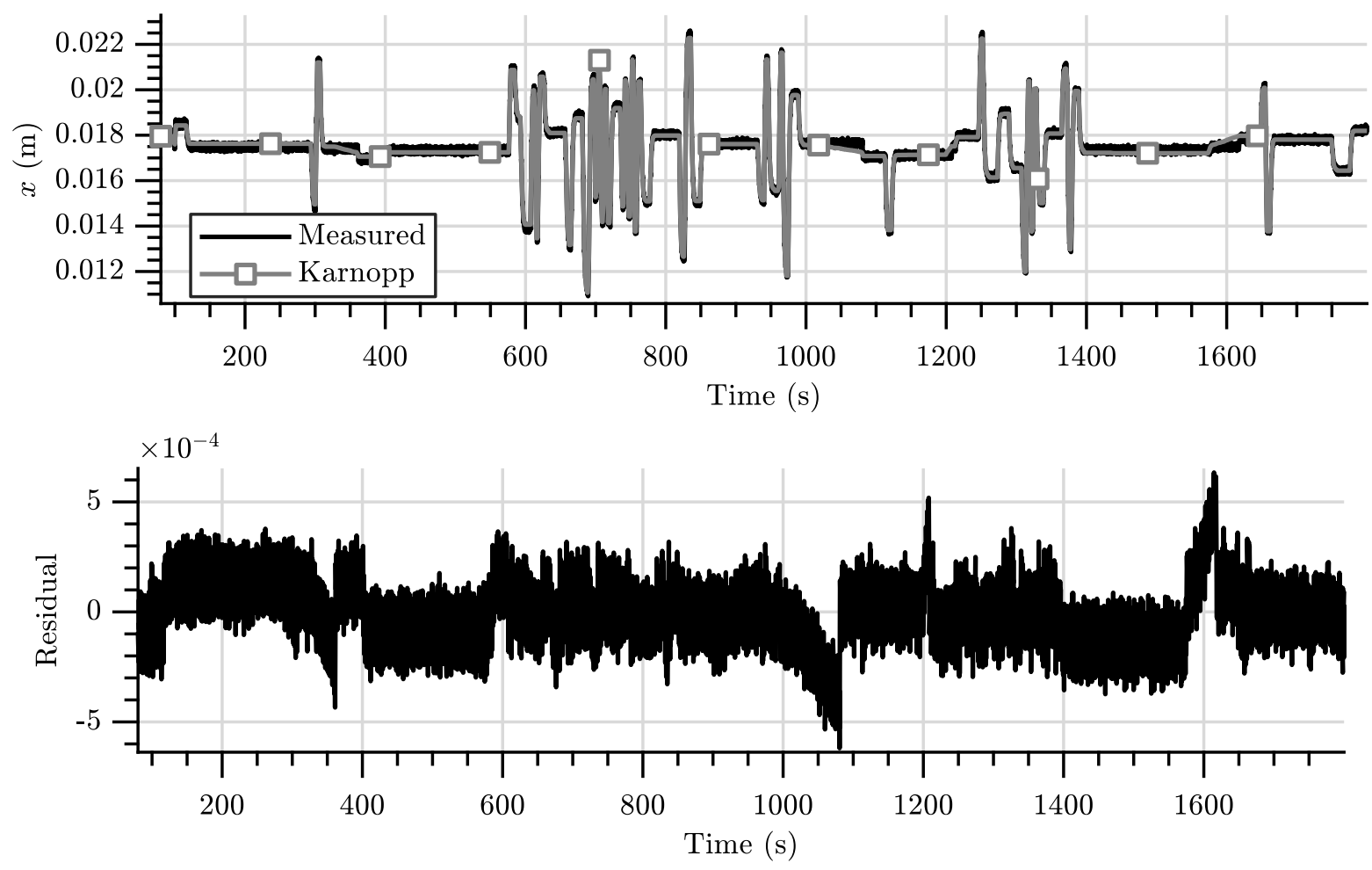

Figure 5.13 - Karnopp model graphite gasket valve residual with model parameters estimated in closed loop using the estimation dataset.

Source: from Author. 

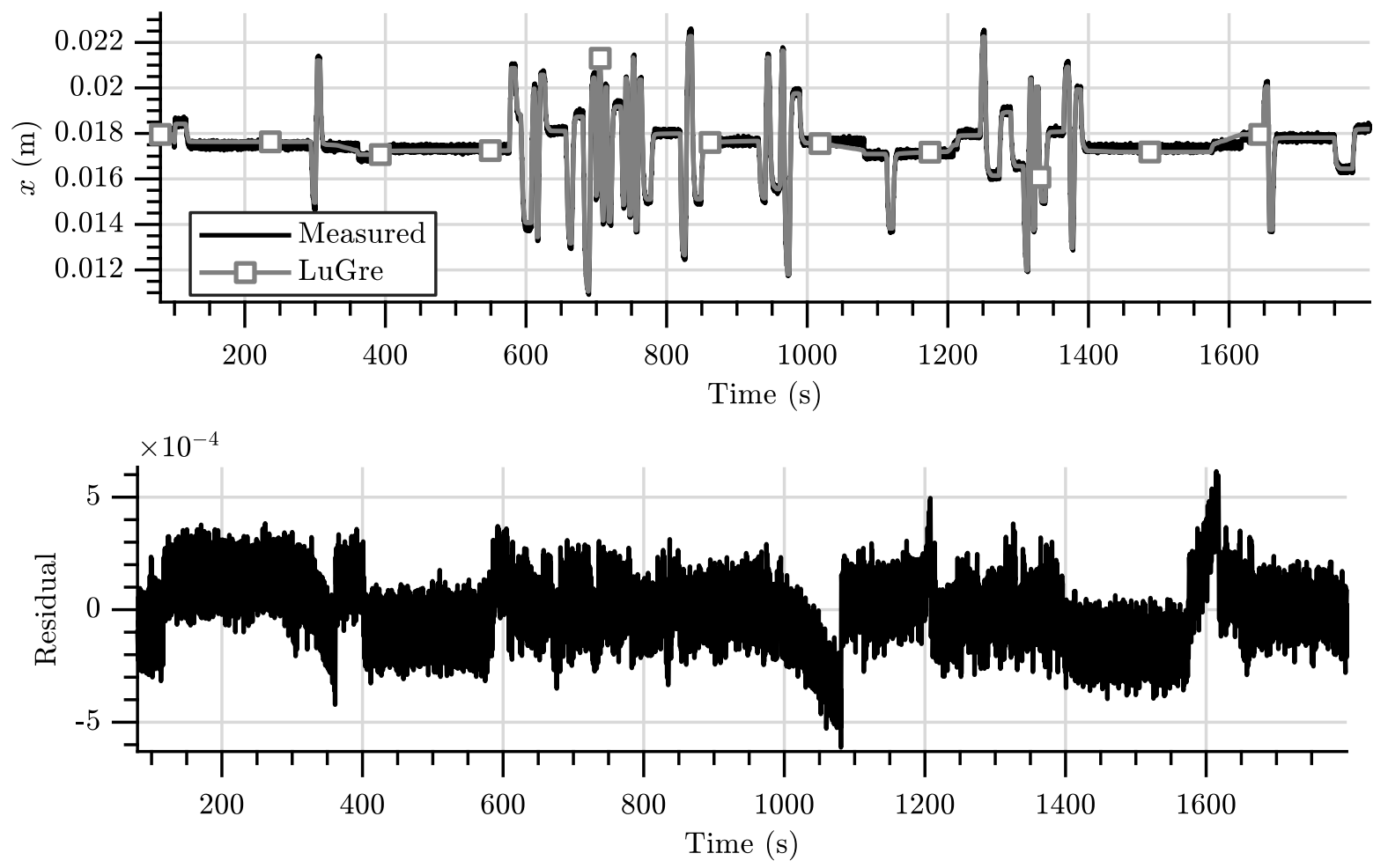

Figure 5.14 - LuGre model graphite gasket valve residual with model parameters estimated in closed loop using the estimation dataset.

Source: from Author.
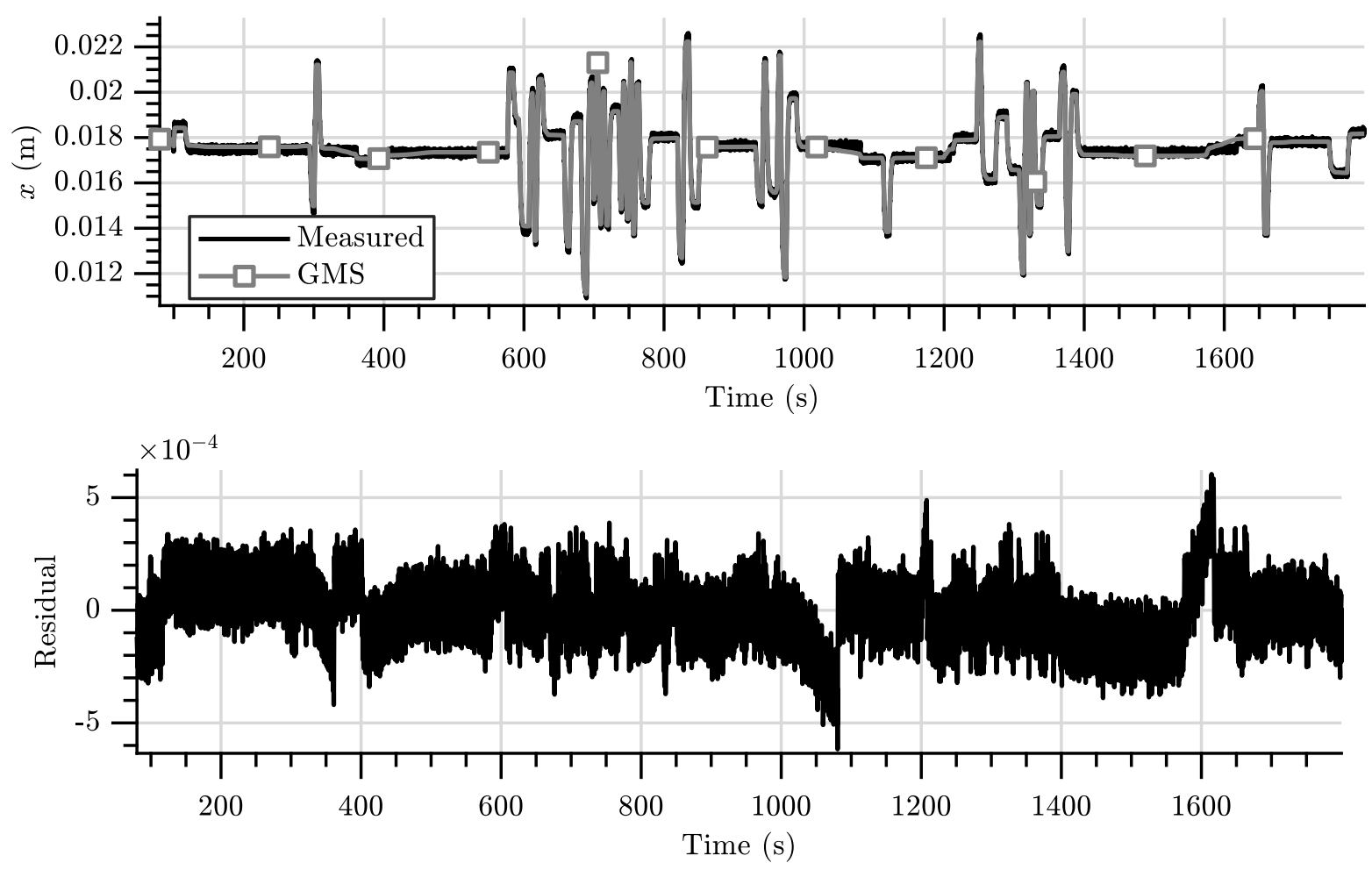

Figure 5.15 - GMS model graphite gasket valve residual with model parameters estimated in closed loop using the estimation dataset.

Source: from Author. 

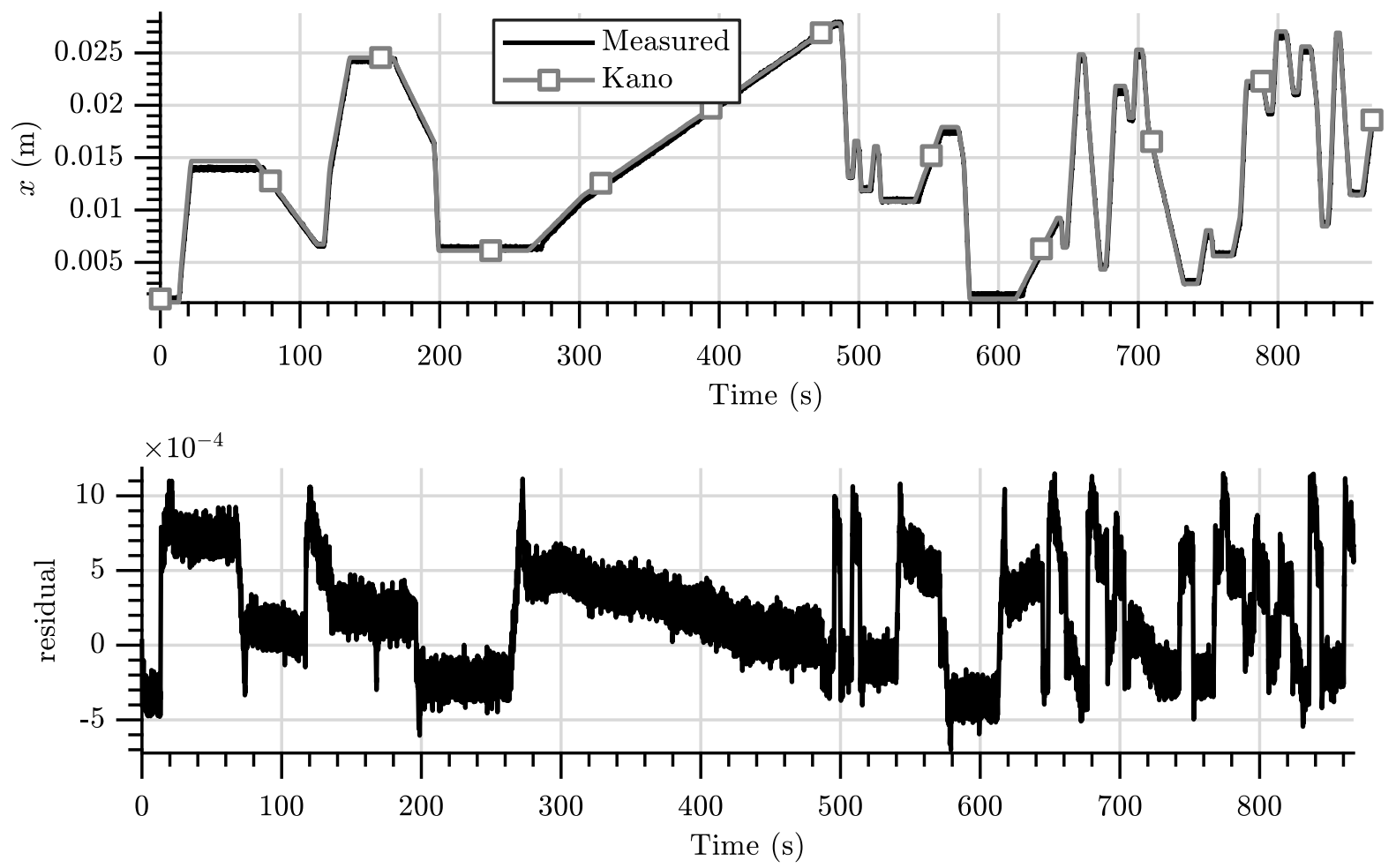

Figure 5.16 - Kano model graphite gasket valve residual with model parameters estimated in closed loop using the validation dataset.

Source: from Author.
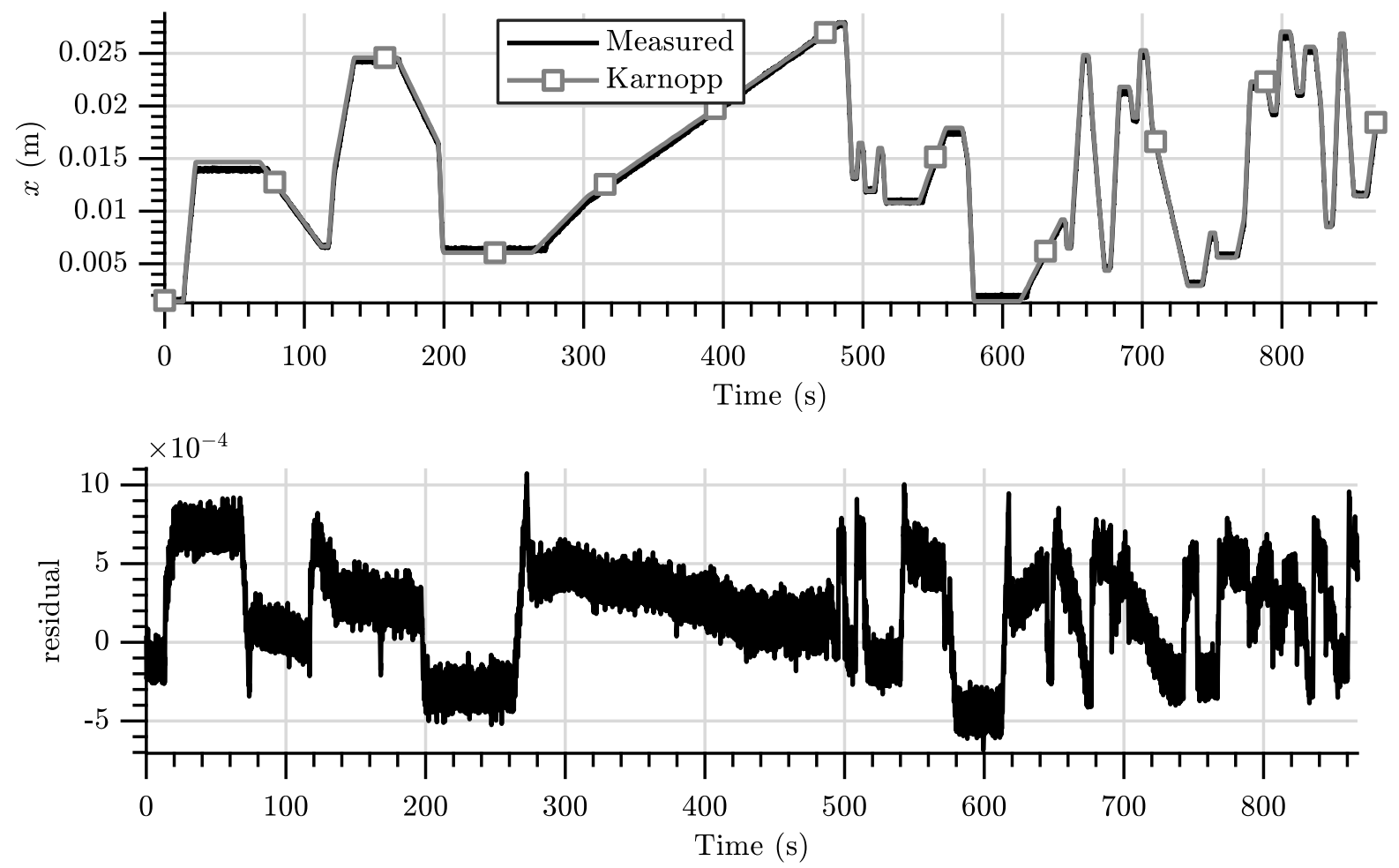

Figure 5.17 - Karnopp model graphite gasket valve residual with model parameters estimated in closed loop using the validation dataset.

Source: from Author. 

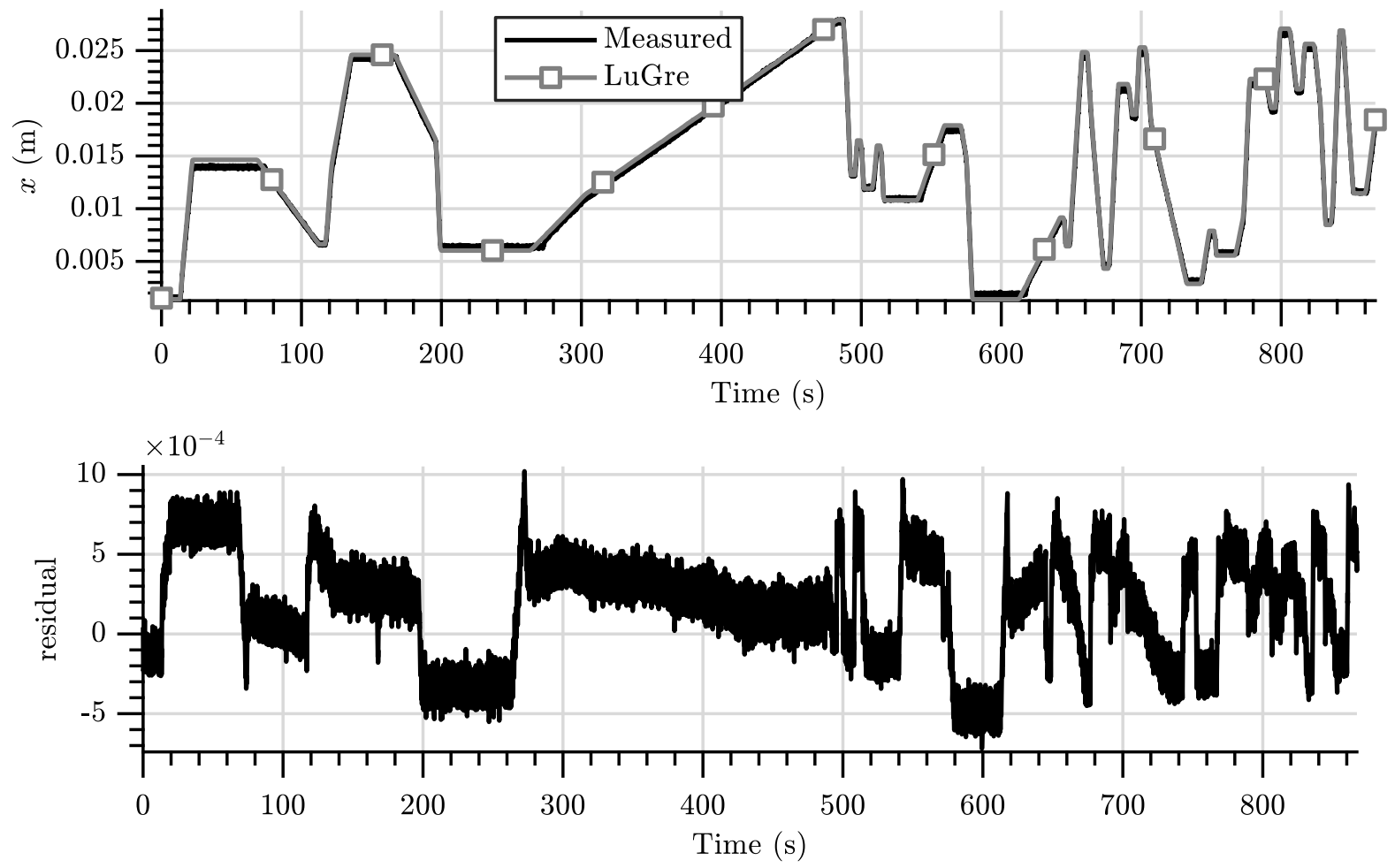

Figure 5.18 - LuGre model graphite gasket valve residual with model parameters estimated in closed loop using the validation dataset.

Source: from Author.
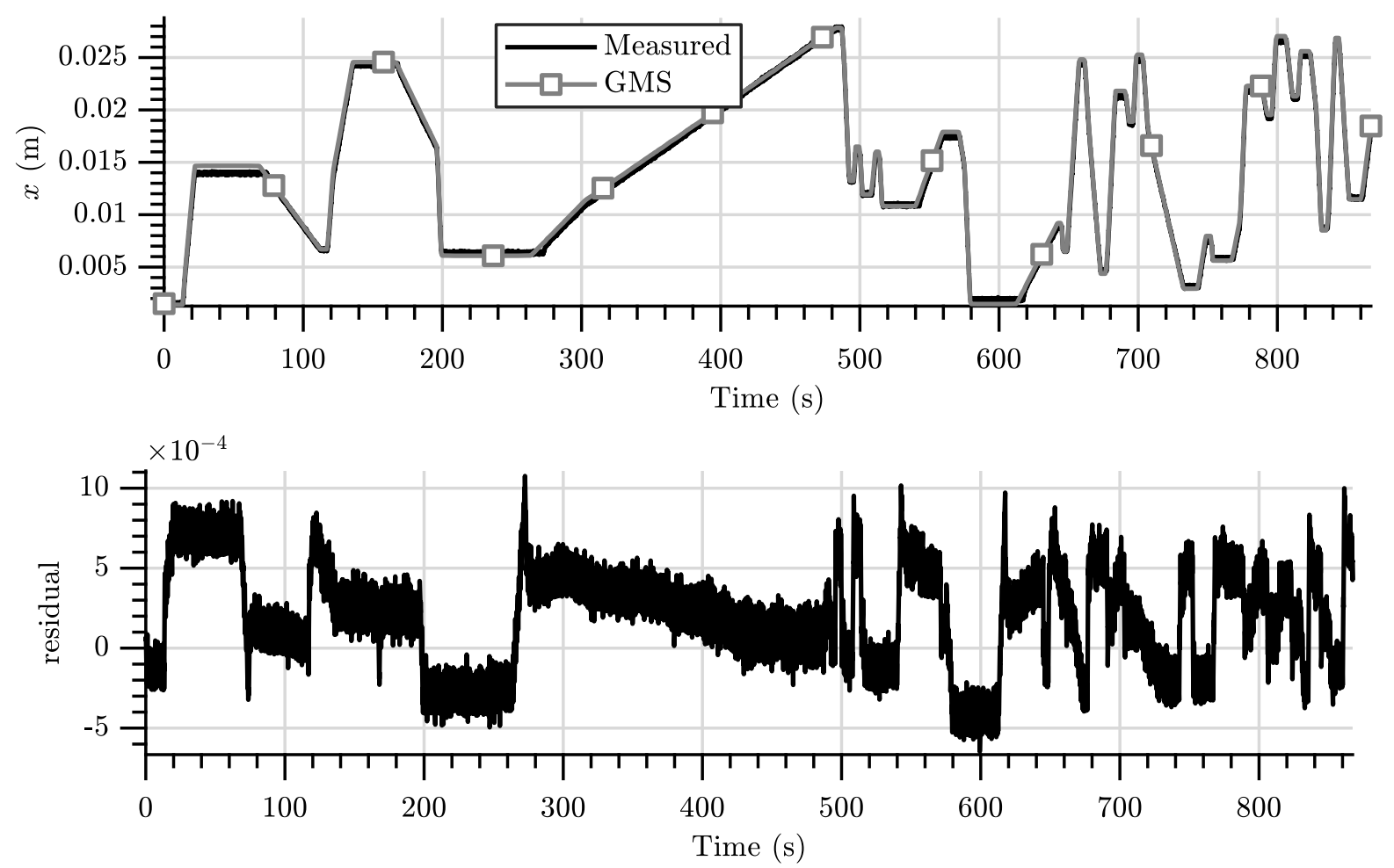

Figure 5.19 - GMS model graphite gasket valve residual with model parameters estimated in closed loop using the validation dataset.

Source: from Author. 


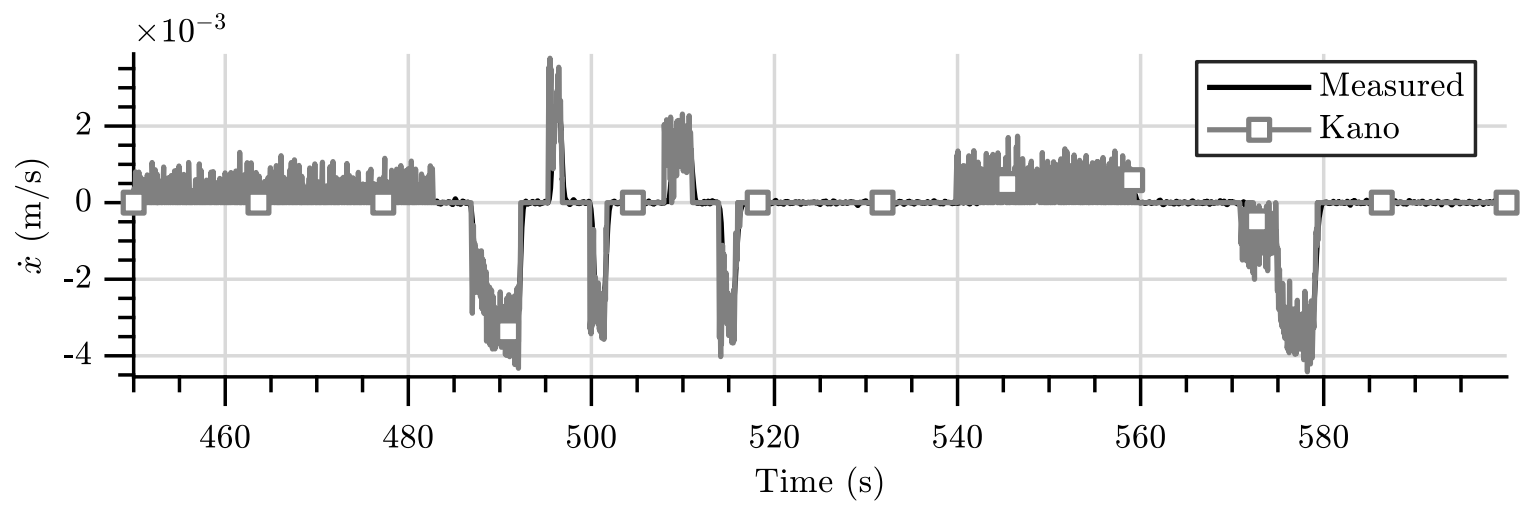

Figure 5.20 - Kano model graphite gasket valve stem velocity prediction (450 s to $600 \mathrm{~s}$ ) with model parameters estimated in closed loop using the open loop validation dataset.

Source: from Author.

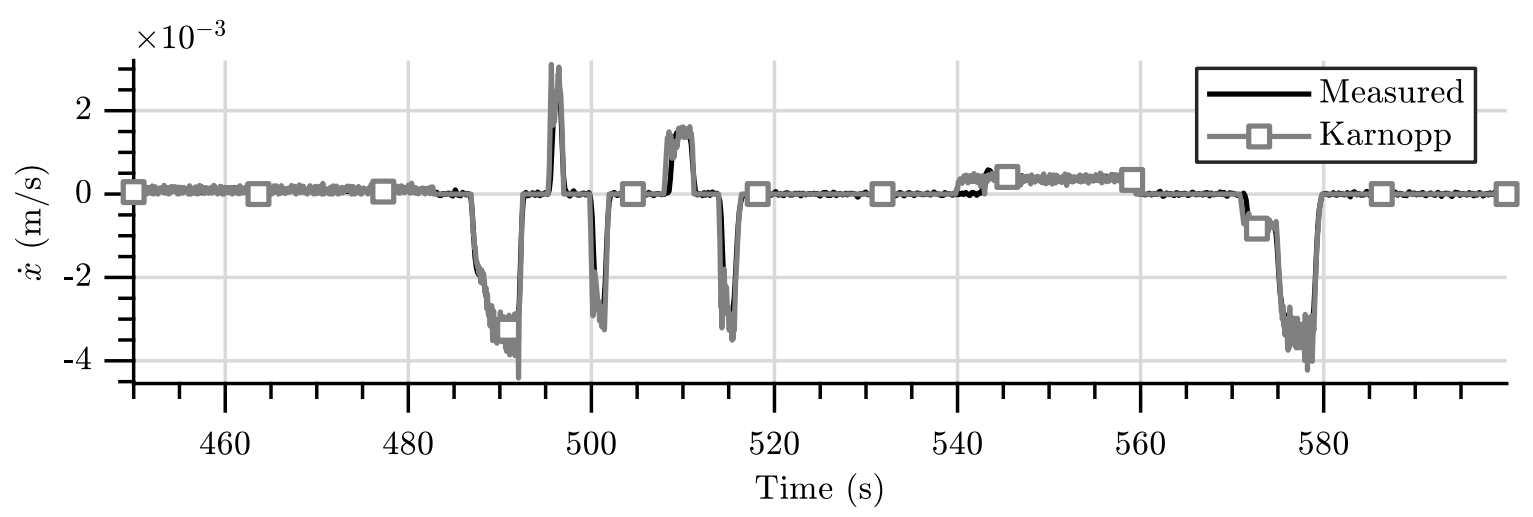

Figure 5.21 - Karnopp model graphite gasket valve stem velocity prediction (450 s to $600 \mathrm{~s}$ ) with model parameters estimated in closed loop using the open loop validation dataset.

Source: from Author.

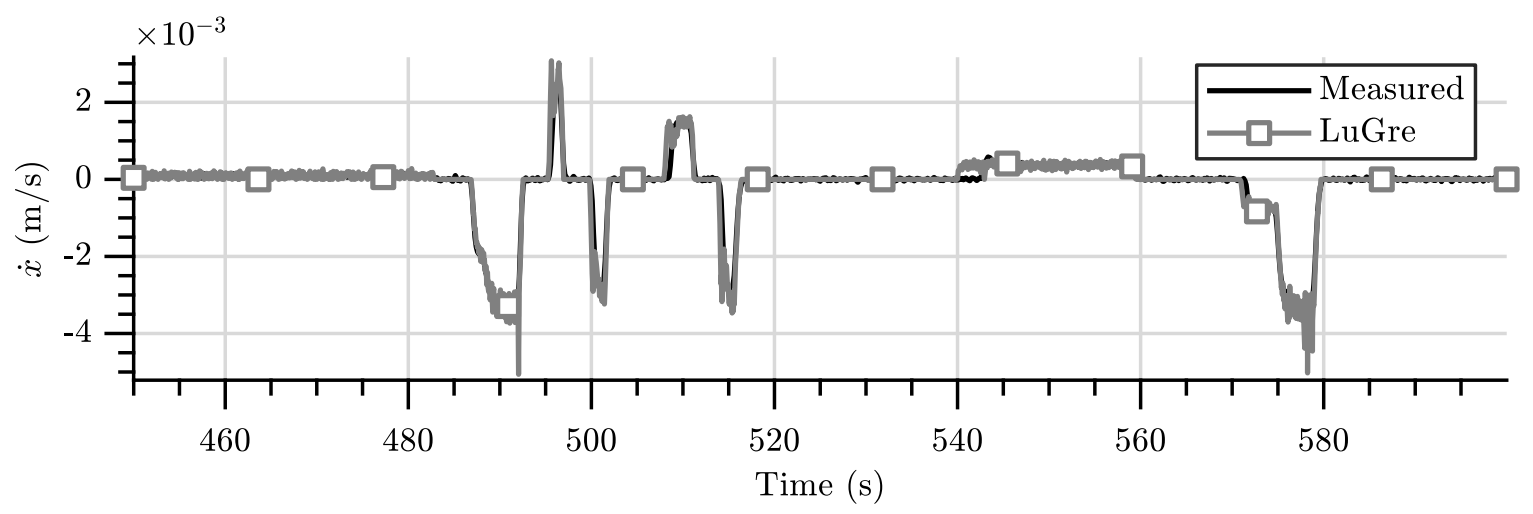

Figure 5.22 - LuGre model graphite gasket valve stem velocity prediction (450 s to $600 \mathrm{~s}$ ) with model parameters estimated in closed loop using the open loop validation dataset.

Source: from Author. 


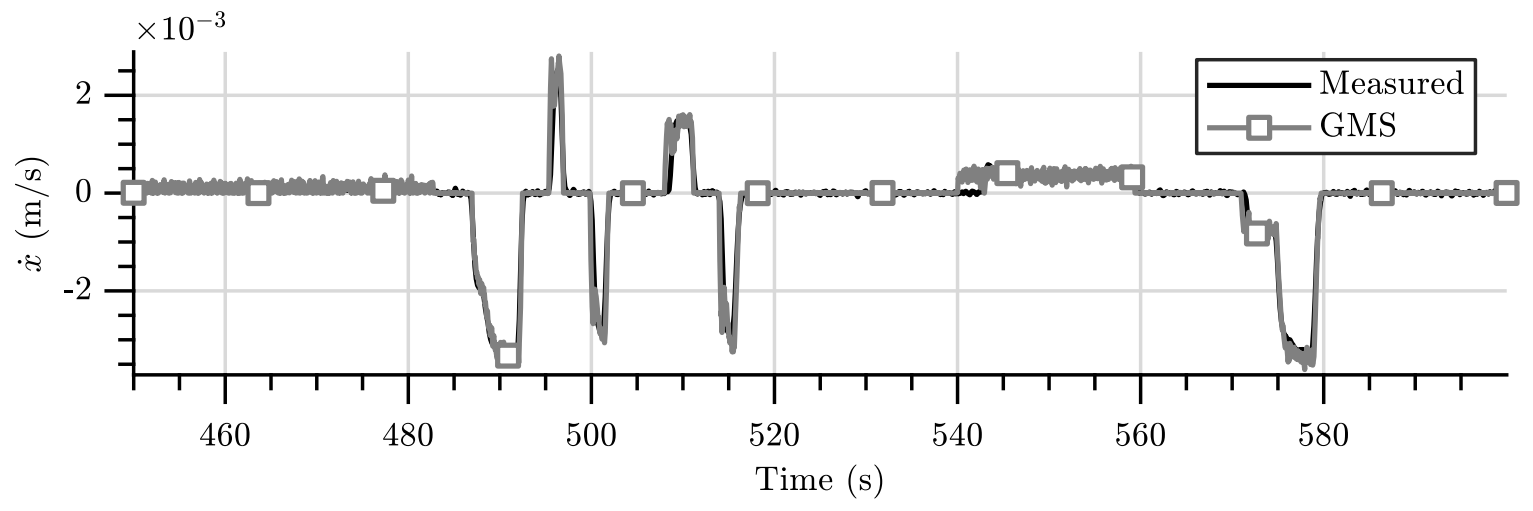

Figure 5.23 - GMS model graphite gasket valve stem velocity prediction (450 s to $600 \mathrm{~s}$ ) with model parameters estimated in closed loop using the open loop validation dataset.

Source: from Author.

The correlation coefficient of some variables are presented in Table 5.16. The correlation coefficients between measured and simulated stem position and velocity are slightly lower for the Kano model, while the other models presented pretty similar results. In general, the stem position and velocity prediction accuracies are very good, for all models. The correlation coefficient between the residual and the simulated stem position and velocity can be considered appropriate, even though some models present higher values in certain cases.

Table 5.16 - Statistical measures of the graphite gasket valve prediction using parameters estimated in closed loop.

\begin{tabular}{lrrrr} 
Model & $r(x, \hat{x})$ & $r\left(e_{x}, \hat{x}\right)$ & $r\left(e_{x}, \hat{\dot{x}}\right)$ & $r(\hat{\dot{x}}, \dot{x})$ \\
\hline Kano & 0.9971 & -0.2145 & 0.4949 & 0.9481 \\
Karnopp & 0.9979 & -0.2856 & 0.0146 & 0.9876 \\
LuGre & 0.9978 & -0.2741 & 0.0111 & 0.9897 \\
GMS & 0.9981 & -0.3216 & -0.0165 & 0.9899 \\
\hline
\end{tabular}

\begin{tabular}{lrrrr} 
Model & $r(x, \hat{x})$ val. & $r\left(e_{x}, \hat{x}\right)$ val. & $r\left(e_{x}, \hat{\dot{x}}\right)$ val. & $r(\hat{\dot{x}}, \dot{x})$ val. \\
\hline Kano & 0.9989 & 0.3259 & 0.4978 & 0.9236 \\
Karnopp & 0.9993 & 0.4603 & 0.2781 & 0.9817 \\
LuGre & 0.9993 & 0.5044 & 0.2735 & 0.9820 \\
GMS & 0.9992 & 0.3993 & 0.2789 & 0.9812
\end{tabular}

val.: indicates to a statistical quantity calculated using the validation dataset.

Source: from Author.

\subsubsection{Teflon Gasket Model Prediction Analysis}

The model predictions, using the closed loop estimated parameters, are compared to the estimation dataset (closed loop) and validation dataset (open loop), as presented in Figures 5.24 to 5.35. It is possible to verify visually that the Kano model presented higher residuals at the beginning of the validation dataset, whereas the other models presented 
very similar stem position prediction precisions. The Karnopp and LuGre models are the less precise for predicting the stem velocity, as the former has a stick-slip behavior and the latter has some spikes, that are not visible in the dataset.
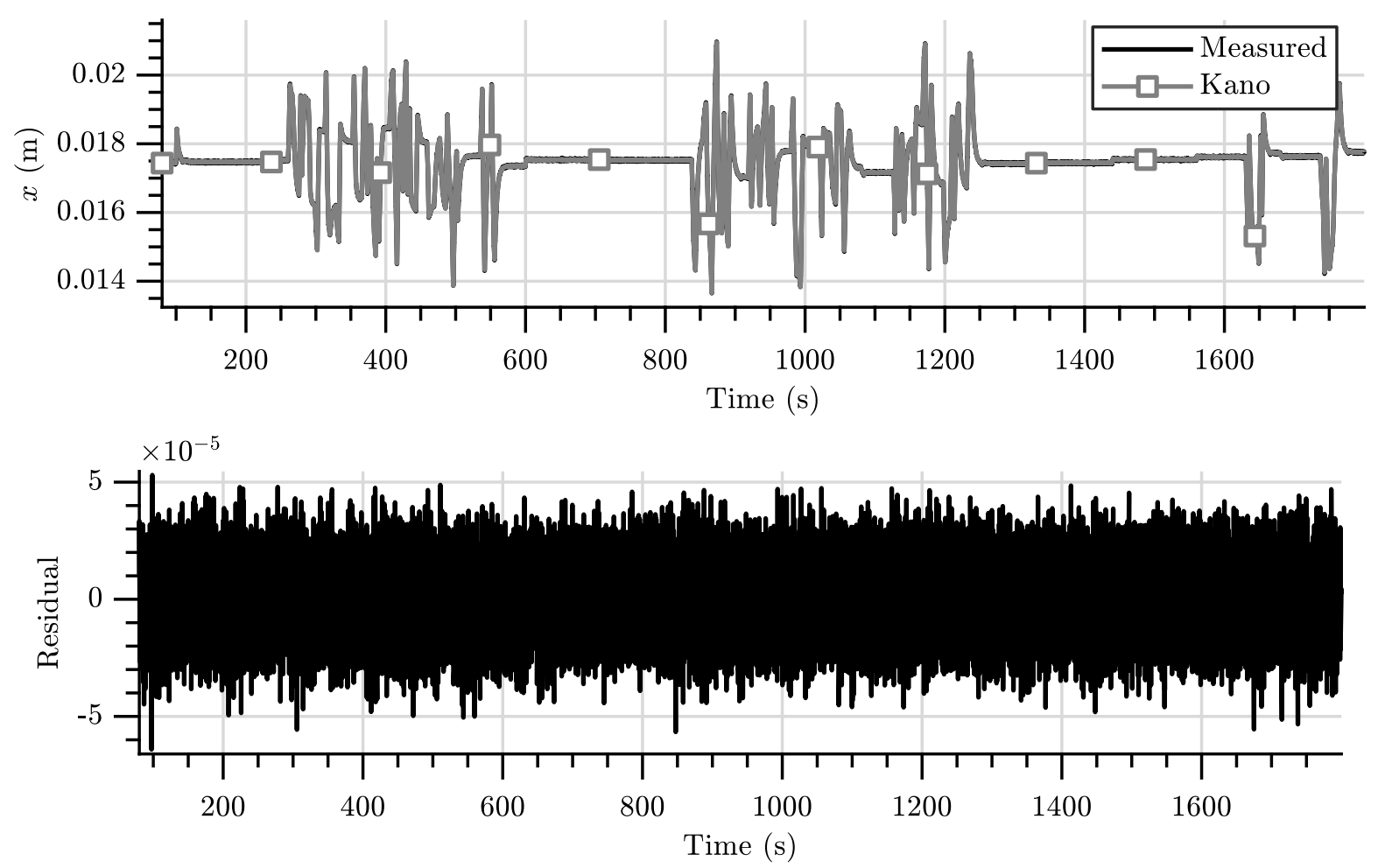

Figure 5.24 - Kano model teflon gasket valve residual with model parameters estimated in closed loop using the estimation dataset.

Source: from Author. 

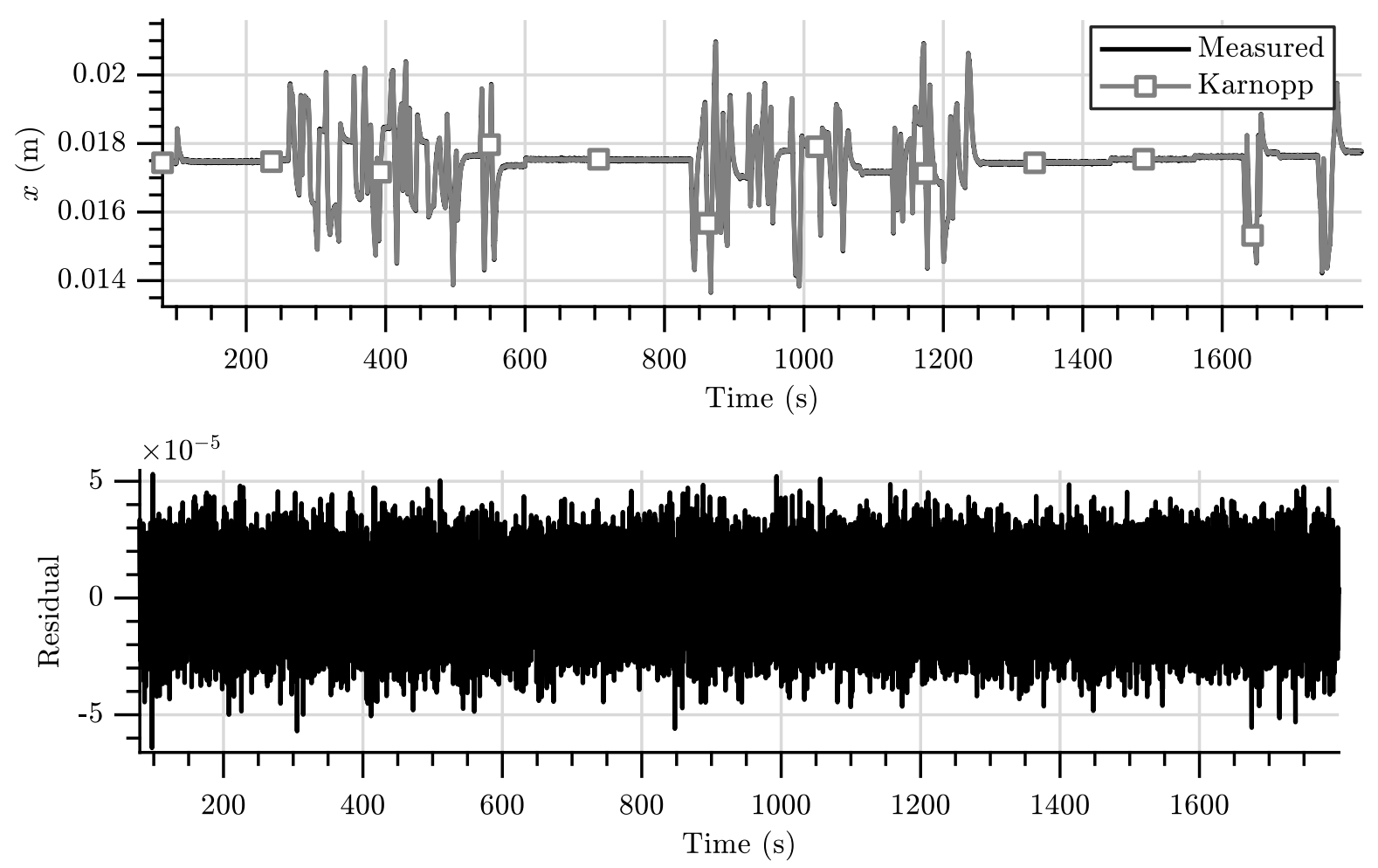

Figure 5.25 - Karnopp model teflon gasket valve residual with model parameters estimated in closed loop using the estimation dataset.

Source: from Author.
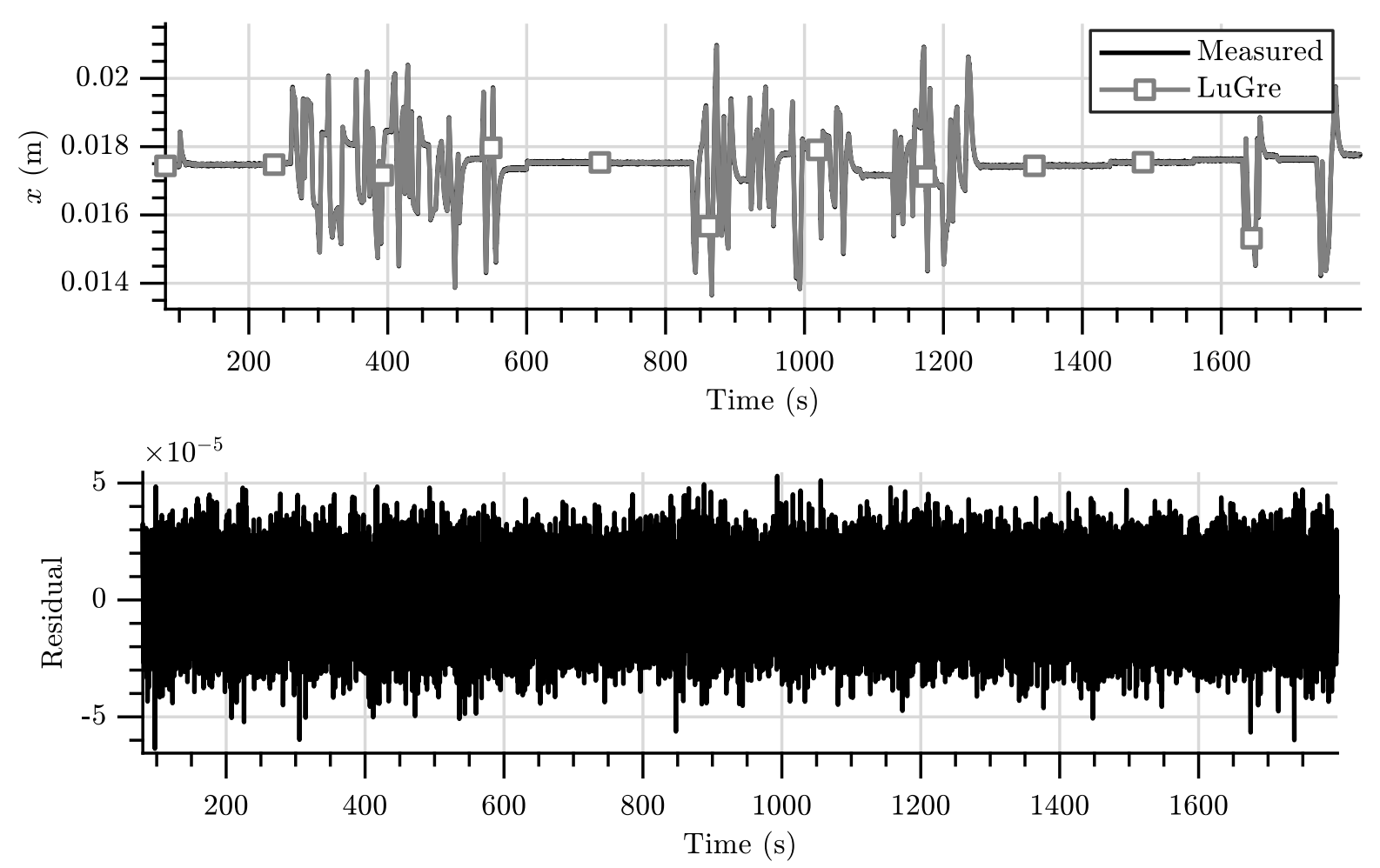

Figure 5.26 - LuGre model teflon gasket valve residual with model parameters estimated in closed loop using the estimation dataset. 

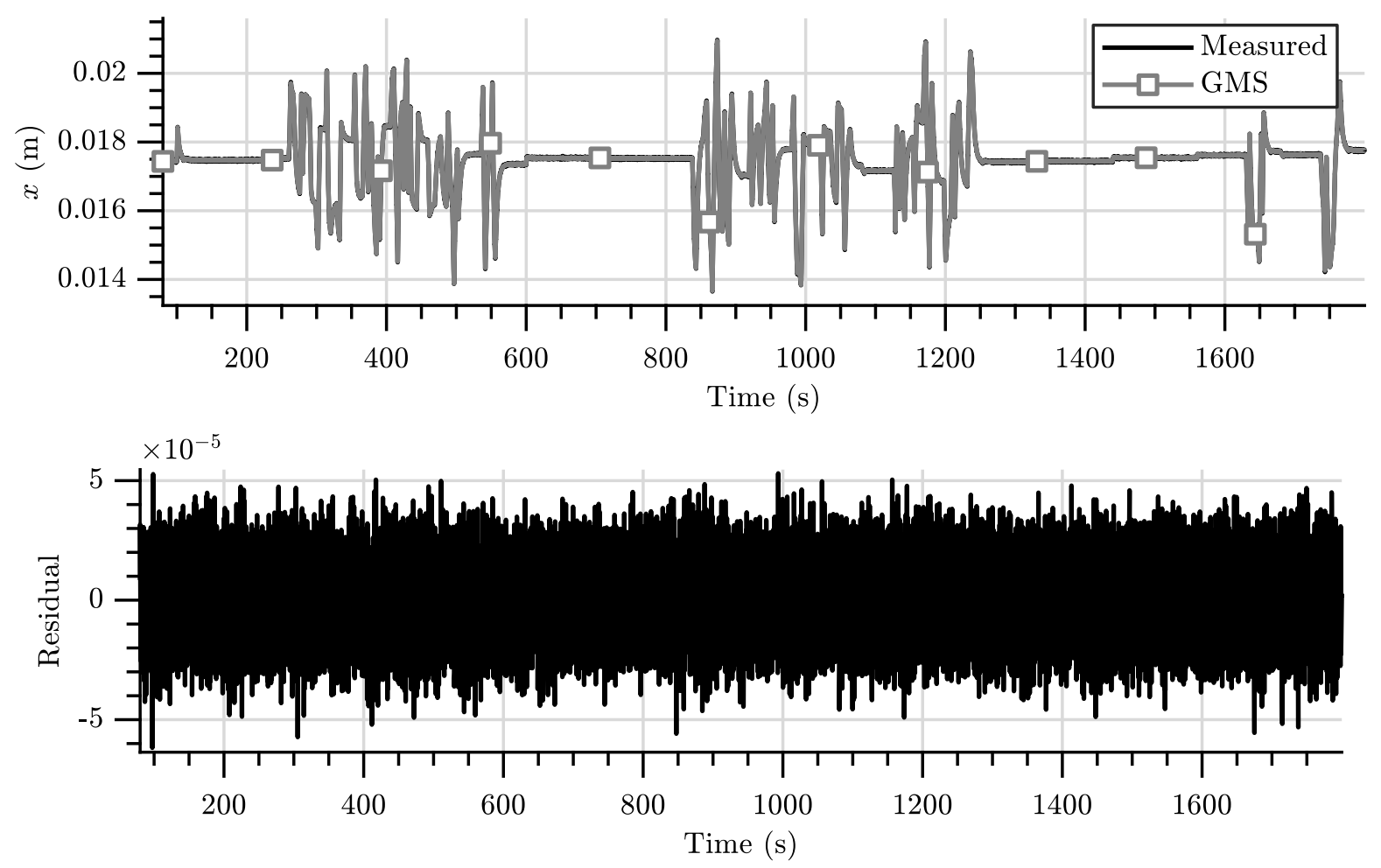

Figure 5.27 - GMS model teflon gasket valve residual with model parameters estimated in closed loop using the estimation dataset.

Source: from Author.
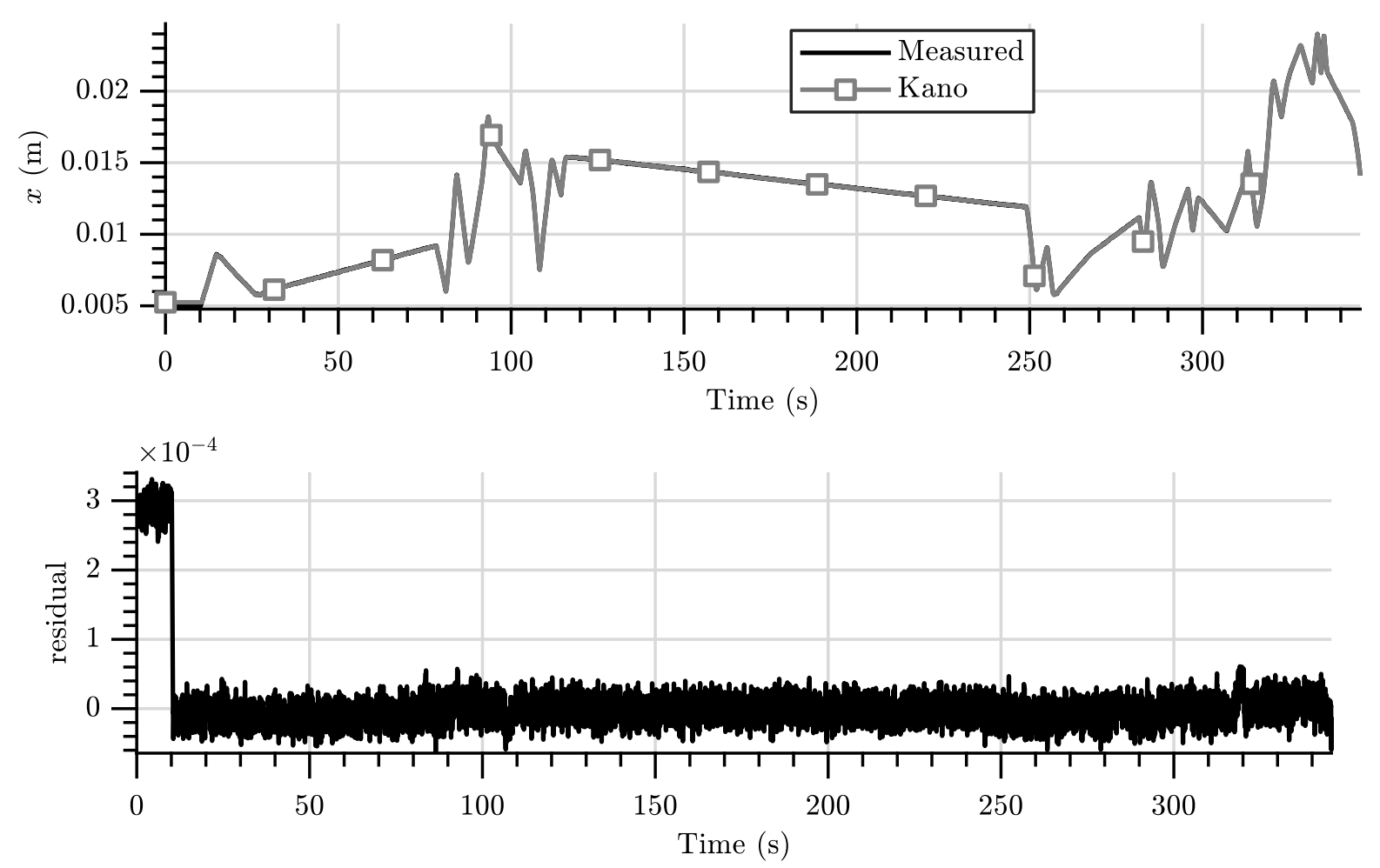

Figure 5.28 - Kano model teflon gasket valve residual with model parameters estimated in closed loop using the validation dataset.

Source: from Author. 

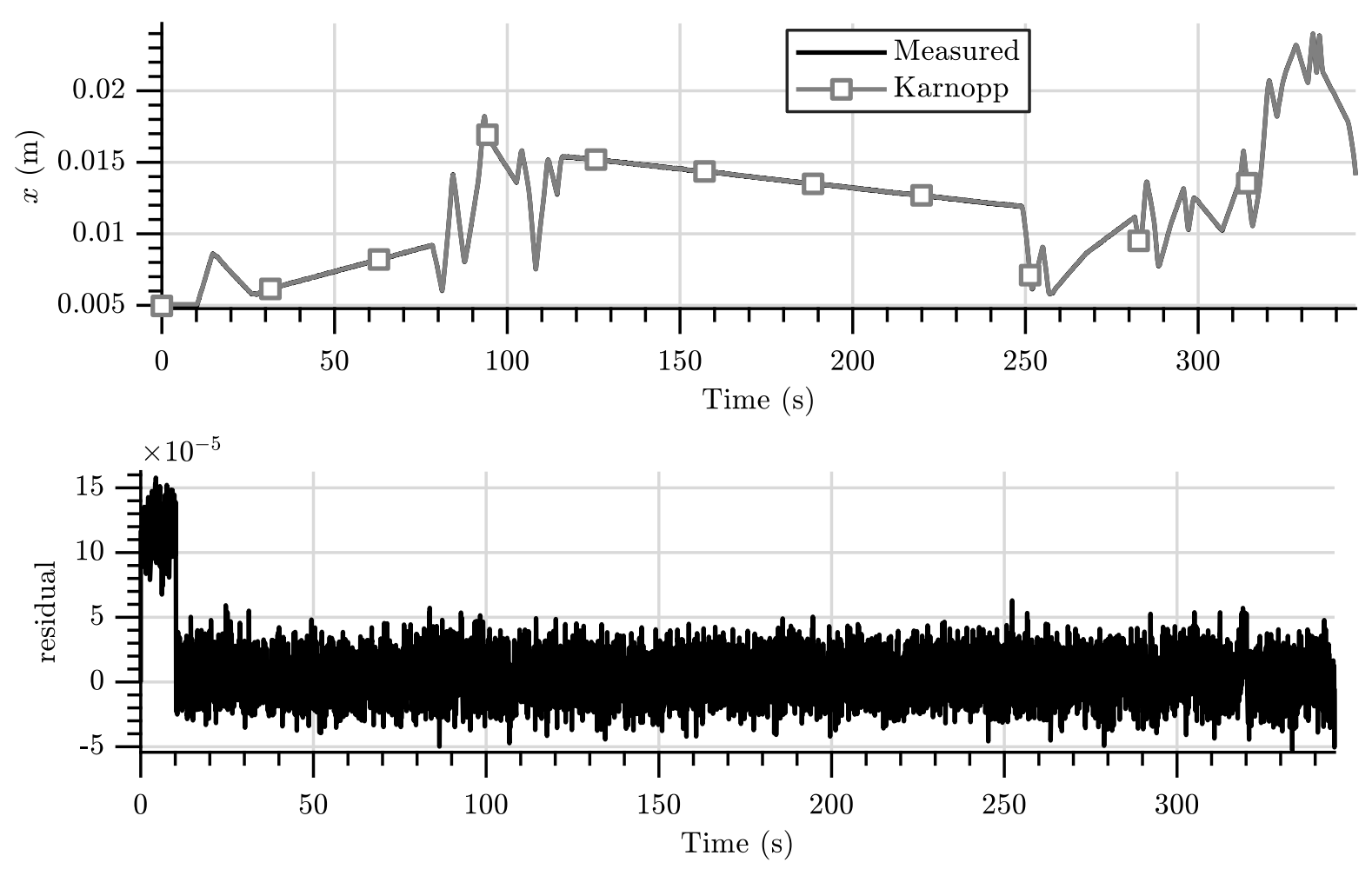

Figure 5.29 - Karnopp model teflon gasket valve residual with model parameters estimated in closed loop using the validation dataset.

Source: from Author.
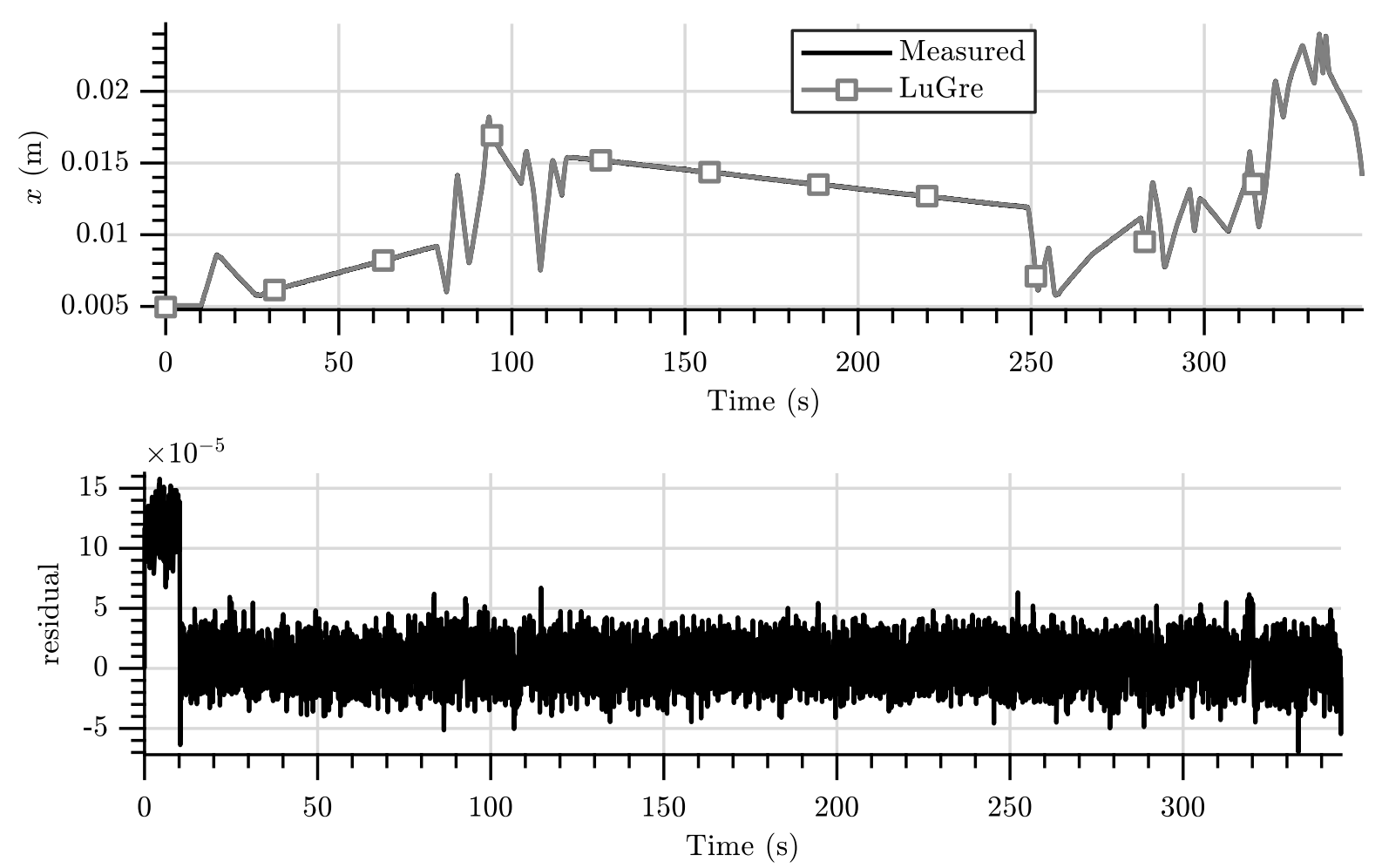

Figure 5.30 - LuGre model teflon gasket valve residual with model parameters estimated in closed loop using the validation dataset.

Source: from Author. 

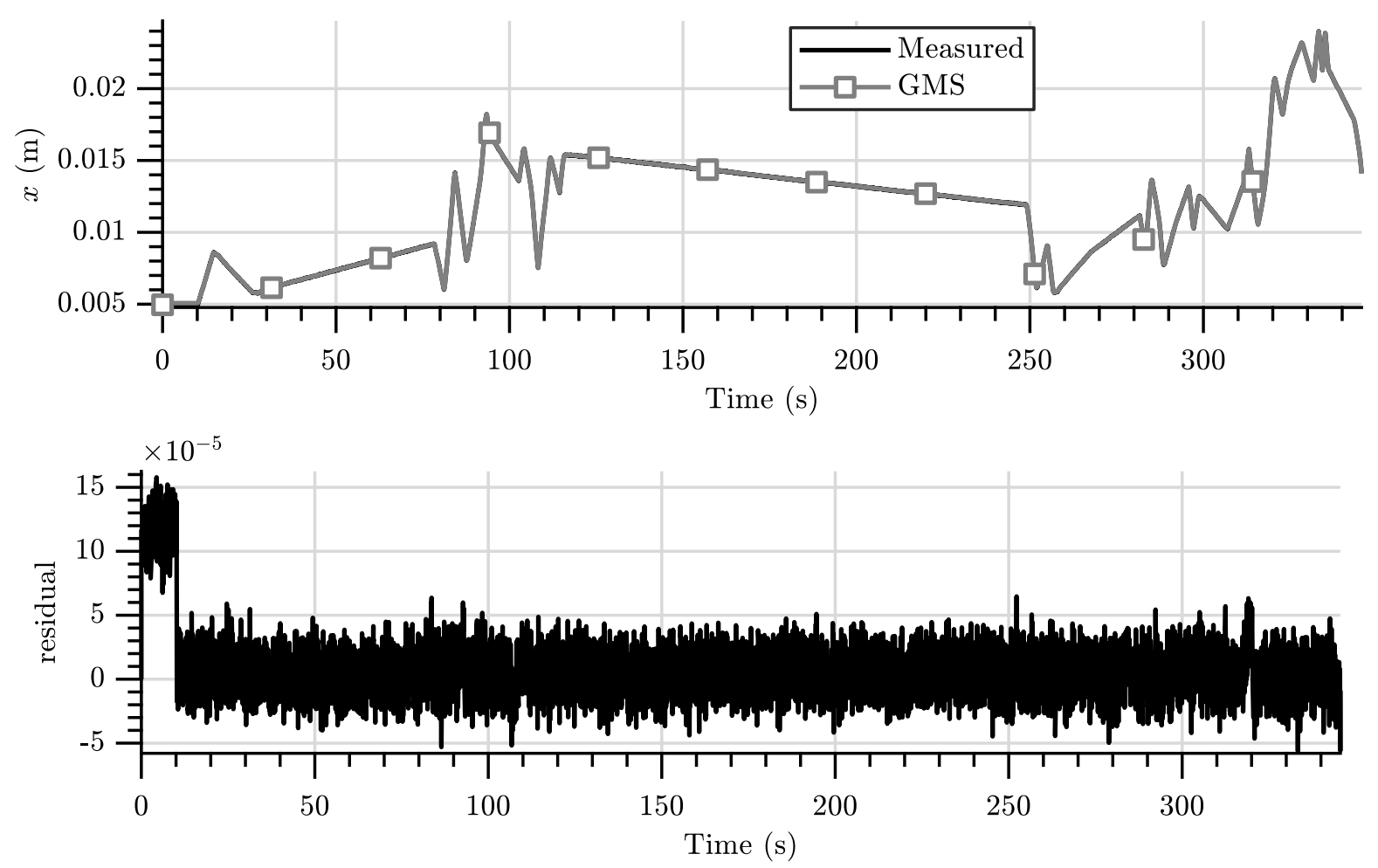

Figure 5.31 - GMS model teflon gasket valve residual with model parameters estimated in closed loop using the validation dataset.

Source: from Author.

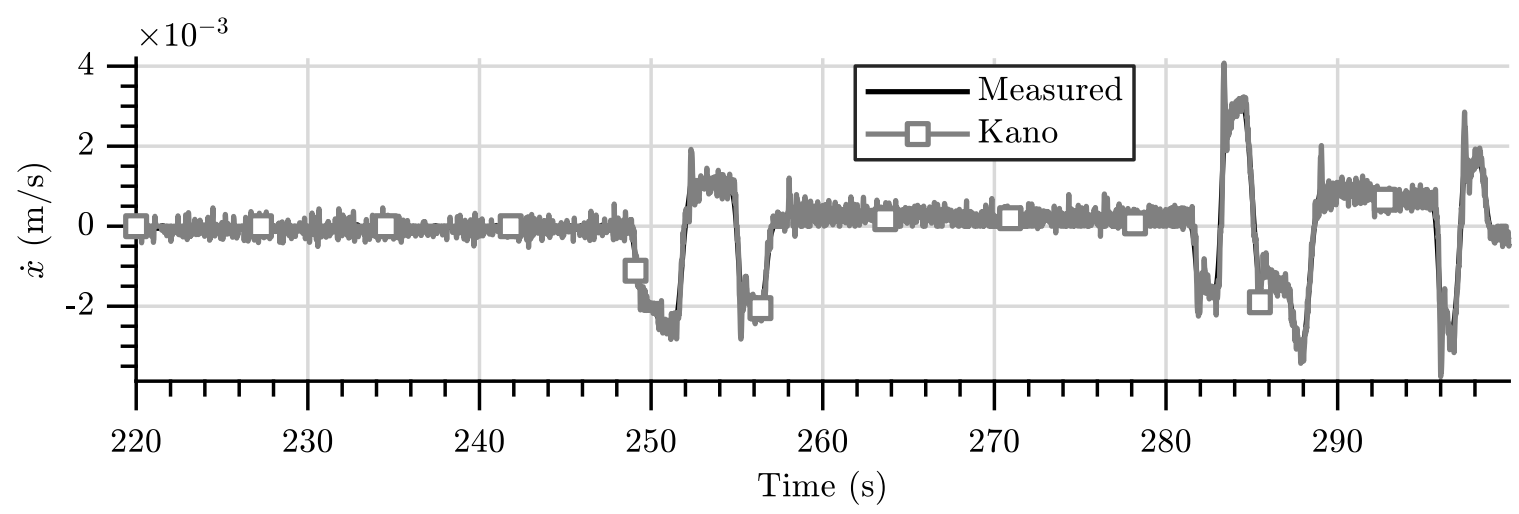

Figure 5.32 - Kano model teflon gasket valve stem velocity prediction (220 s to $300 \mathrm{~s}$ ) with model parameters estimated in closed loop using the open loop validation dataset.

Source: from Author. 


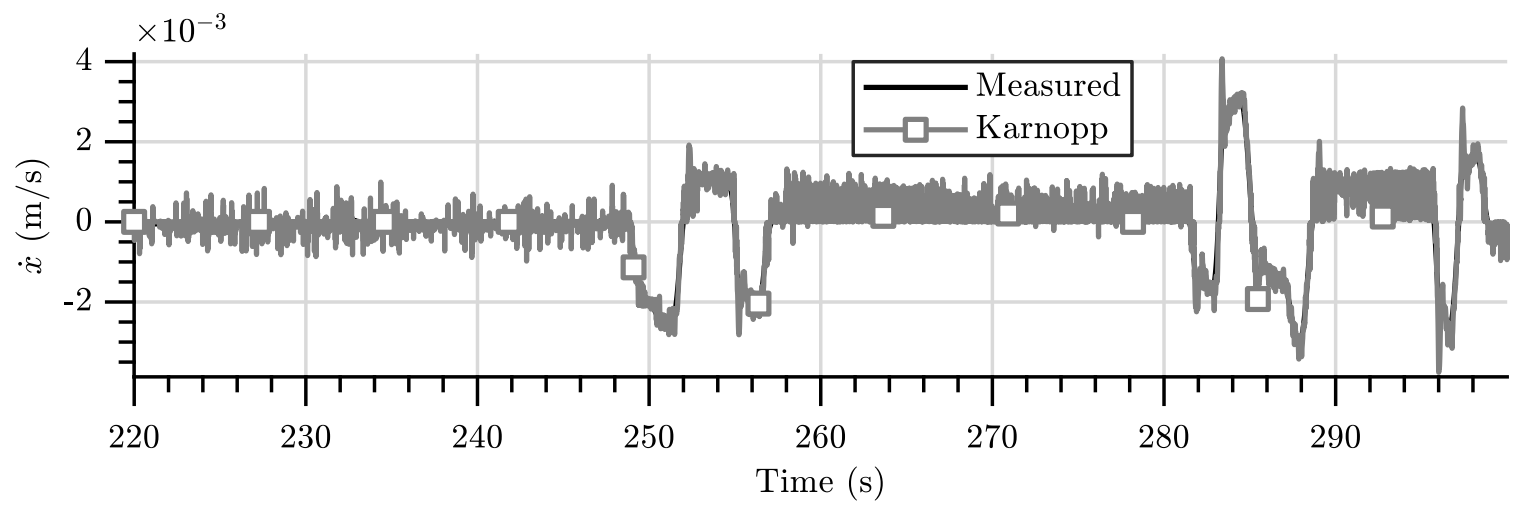

Figure 5.33 - Karnopp model teflon gasket valve stem velocity prediction $(220 \mathrm{~s}$ to $300 \mathrm{~s}$ ) with model parameters estimated in closed loop using the open loop validation dataset.

Source: from Author.

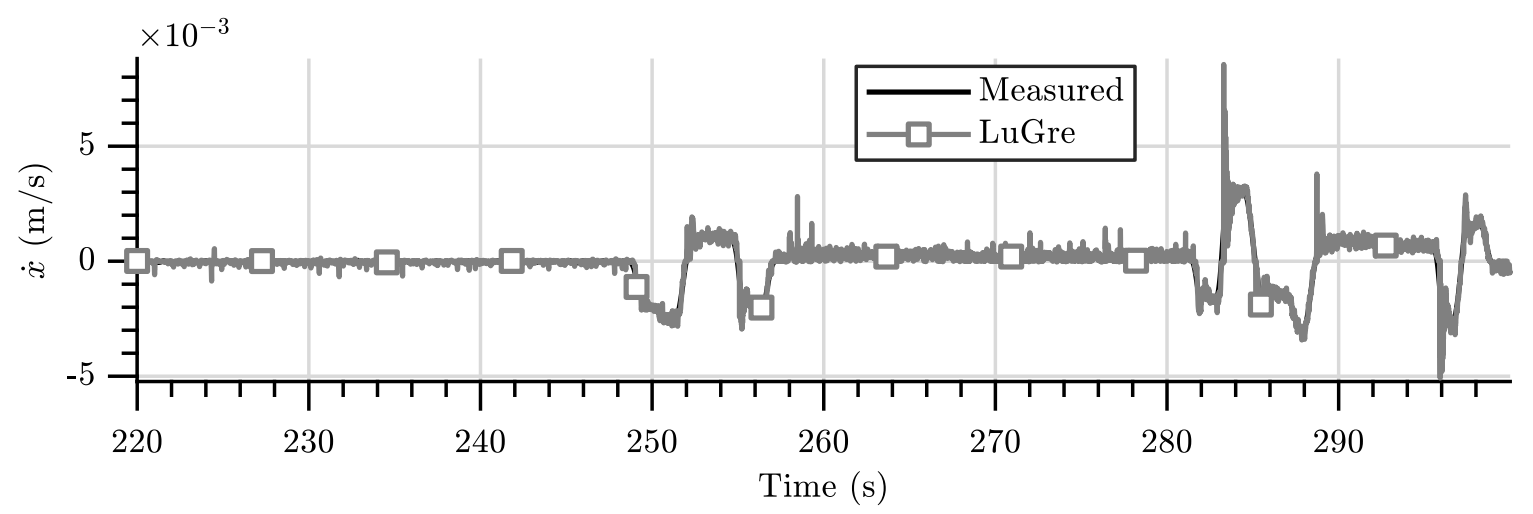

Figure 5.34 - LuGre model teflon gasket valve stem velocity prediction (220 s to $300 \mathrm{~s}$ ) with model parameters estimated in closed loop using the open loop validation dataset.

Source: from Author.

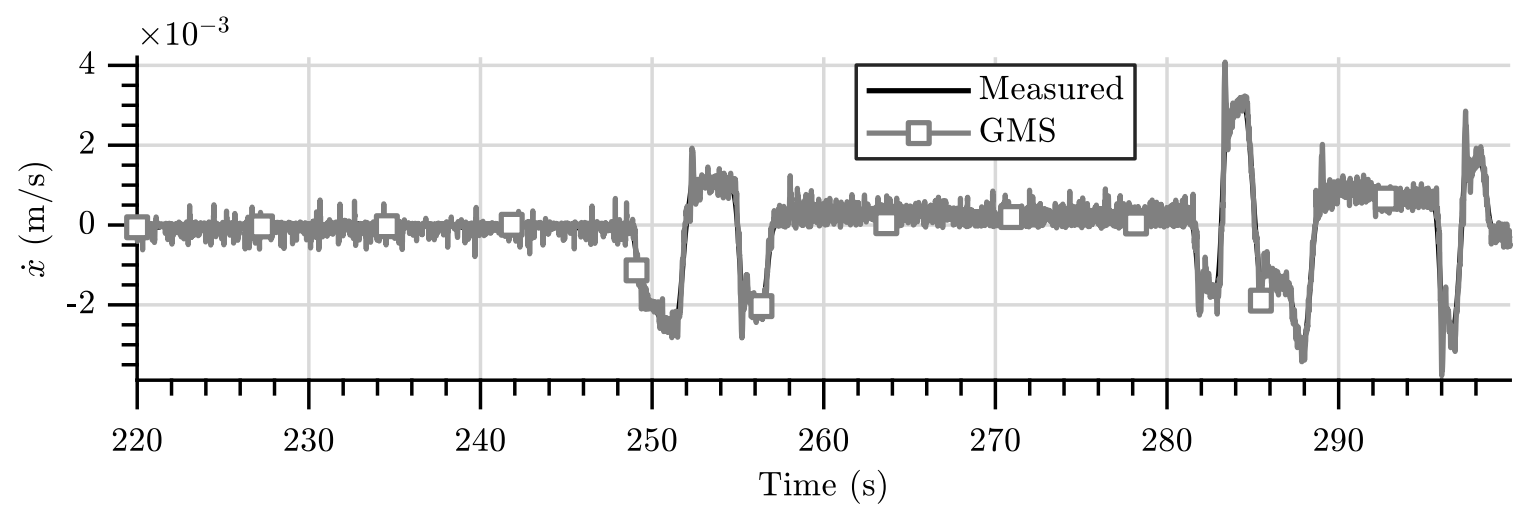

Figure 5.35 - GMS model teflon gasket valve stem velocity prediction (220 s to $300 \mathrm{~s}$ ) with model parameters estimated in closed loop using the open loop validation dataset.

Source: from Author. 
Table 5.17 presents some statistical measures that are used to analyze the prediction precision of the teflon gasket valve model, when its parameters are estimated in closed loop. It is possible to verify that the stem prediction is excellent for every model and dataset. In the estimation dataset, one may note an increase in the correlation coefficient between the residuals and stem position, indicating that the estimation performance could be improved. The most precise models, with respect to the stem velocity prediction, are the Kano and GMS.

Table 5.17 - Statistical measures of the graphite gasket valve prediction using parameters estimated in closed loop.

\begin{tabular}{lrrrr} 
Model & $r(x, \hat{x})$ & $r\left(e_{x}, \hat{x}\right)$ & $r\left(e_{x}, \hat{\dot{x}}\right)$ & $r(\hat{\dot{x}}, \dot{x})$ \\
\hline Kano & 0.99990 & -0.6206 & -0.0208 & 0.9596 \\
Karnopp & 0.99996 & -0.4717 & -0.0252 & 0.9329 \\
LuGre & 0.99996 & -0.4720 & -0.0264 & 0.9333 \\
GMS & 0.99996 & -0.4748 & 0.0229 & 0.9595 \\
\hline
\end{tabular}

\begin{tabular}{lrrrr} 
Model & $r(x, \hat{x})$ val. & $r\left(e_{x}, \hat{x}\right)$ val. & $r\left(e_{x}, \hat{\dot{x}}\right)$ val. & $r(\hat{\dot{x}}, \dot{x})$ val. \\
\hline Kano & 0.99992 & -0.1880 & 0.0258 & 0.9797 \\
Karnopp & 0.99998 & -0.2665 & 0.0353 & 0.9702 \\
LuGre & 0.99998 & -0.2514 & 0.0686 & 0.9632 \\
GMS & 0.99998 & -0.2598 & 0.1025 & 0.9792 \\
\hline
\end{tabular}

val.: indicates to a statistical quantity calculated using the validation dataset.

Source: from Author.

\subsection{Valve Model Selection}

The models presents very similar overall characteristics to predict the valve stem position, but each have singularities. In this section the results obtained so far are discussed and a valve model is selected for the rest of this work.

The Kano model is very sensitive to noise, because it has a flat passband, responding to every excitation frequency (limited by the model sampling time), as seen in Figure 4.20. If the Kano model has slip band, then it is inevitable that it presents stick-slip behavior if the excitation signal has noise. This is undesirable, since it may affect negatively the $C_{p}$ design, as the slip band is a major player in inducing control loops limit cycles (MOHAMMAD; HUANG, 2012). The great advantage of using Kano model is the computational efficiency.

The Karnopp model presents an overall good stem position prediction, but it may predict a stick-slip behavior when it is not present in the plant. Although the Karnopp model holds the good stem velocity prediction indexes, visually some imprecisions can be seen even in simple cases (with low friction index), as shown in Figure 5.21. Therefore, the same drawback regarding $C_{p}$ design with Kano model is applied to the Karnopp model. This drawback, however, has less severity for the Karnopp model, since there are parameter combinations that may eliminate the stick-slip behavior. One Karnopp model advantage 
is the possibility to use a higher time step than LuGre and GMS models, improving the computational efficiency, however, it would deteriorate even more the stick-slip prediction accuracy.

The LuGre model is very precise to model pneumatic diaphragm valves when the excitation signal is filtered and with high signal to noise ratio. However, this model has a high risk of presenting terrible predictions if the input signal is deteriorated, as seen in Figure 4.21. It is prohibitive to use a model which has an increased mathematical complexity, when compared to the Kano and Karnopp models, that may fail if some combination of events happen.

The GMS model is very precise in every experimental tests that were carried out, but its high number of parameters, some of them with extremely low sensitivity for the stem position prediction, prevents a perfect estimation even in the ideal case. Also, estimating this model parameters is the most time consuming, among the tested models. It is worth mentioning, though, that the computational performance can be greatly improved by using Field Programmable Gate Array (FPGA) and fixed point calculations. It is possible to simulate $1 \mathrm{~ms}$ with one FPGA clock, which is around $100 \mathrm{MHz}$. Therefore, a 30 min simulation would take $0.018 \mathrm{~s}$, which is significantly lower than the needed $50 \mathrm{~s}$ to simulate using a personal computer.

The selected models, to be used for the automatic friction compensation part of this thesis, are the Kano, Karnopp and GMS models. The Kano model certainly has its limitations, however, so does the Karnopp and LuGre models, therefore it is reasonable to select the most computationally efficient model among these three. The Karnopp model is selected as it has consistent estimation performance and has better predictions than the Kano model. With respect to the GMS model, it is selected as it has pretty good stem position and velocity prediction precision and it is robust with respect to diaphragm pressure measurement noise. In the following developments of this thesis, it will be possible to verify if the added precision of the GMS model produces more efficient valve position controllers, when compared to the Kano and Karnopp models. 


\section{Automatic Friction Compensation}

In this chapter, the estimated valve models obtained in Chapters 4 and 5 are used in an Adaptive Inverse Control (AIC) framework, where the control system parameters are tuned using an optimization algorithm that runs offline, as presented in Figure 6.1. The idea is to tune the $C_{p}$ parameters so that the plant has the same closed loop response as the reference model.

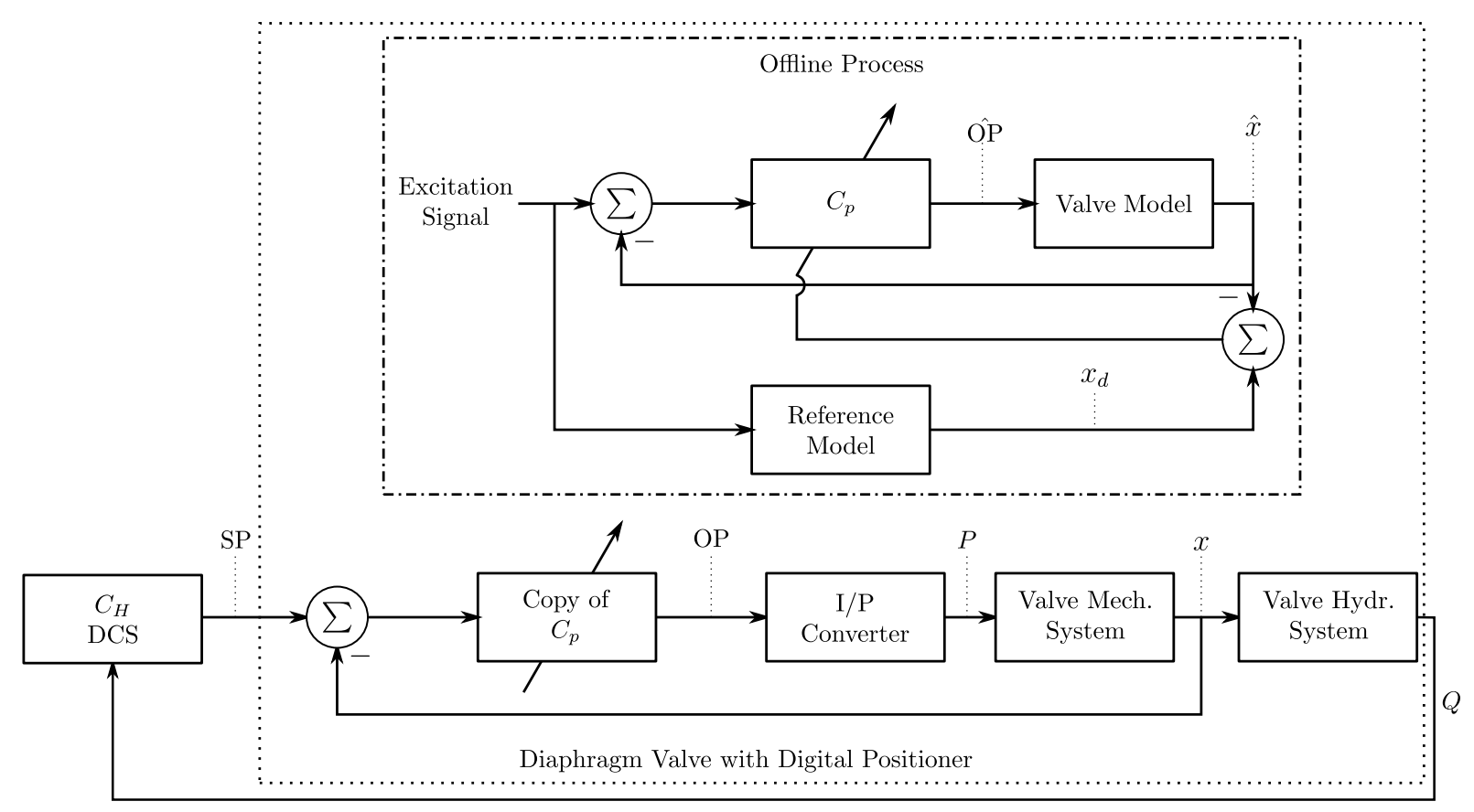

Figure 6.1 - Adaptive Inverse Control framework example for the diaphragm valve position controller tuning.

Source: based on Widrow and Walach (2008).

If the plant corresponds to a stable linear time invariant system, then Widrow and Walach (2008) show that it is possible to invert the plant dynamics using a Finite Impulse Response Filter, with parameters tuned using the Recursive Least Squares algorithm. However, if the plant is nonlinear, then there is not a fixed generic structure that is able to deliver a controller that can approximate any plant to the desired closed loop response $\left(x_{d}\right)$. Widrow and Walach (2008) suggest that, for nonlinear systems, the controller can be a Volterra filter or a neural network, however, as there are many control structures that were suggested in the friction compensation literature, some of these compensators are employed instead. 


\subsection{The AIC framework}

One aspect that is very important to construct a good AIC framework is to select a proper reference model, in a sense that the valve model is able to exhibit such output response with $C_{p}$. In the diaphragm control valve case, the reference model is easily derived, as the plant does not present any substantial dead time and it is a stable system. The reference model is designed to be a first order filter of the excitation signal, as it is not desirable that the valve presents any movement reversal, since it would mean that $C_{p}$ would have to overcome the friction force more than once to reach the desired response. The first order filter time constant is chosen to be $50 \%$ lower than the I/P model time constant. This choice is based on the trade-off between a fast valve response and the control effort, as faster desired valve responses mean higher control efforts.

The excitation signal is also very important to obtain a good $C_{p}$ tuning. It has to present the characteristics in which the controller is expected to perform during its regular operation. As the diaphragm valve with digital positioner is a cascade control loop, the excitation signal is rather stochastic and depends on how the hydraulic system and $C_{H}$ are designed. Therefore, the excitation signal is a series of random ramps (commonly found in integrative controllers) and steps. The ramp velocities, $v_{e x c C_{p}}$, is given by a Gaussian random variable, with a standard deviation of:

$$
\operatorname{std}\left(v_{e x c C_{p}}\right)=\frac{50}{5 \hat{\tau}_{\mathrm{I} / \mathrm{P}}}
$$

with zero mean and a sampling time of:

$$
T_{v_{e x c} C_{p}}=\frac{S_{0} / 2}{\operatorname{std}\left(v_{e x c} C_{p}\right)} .
$$

The steps have a mean value of $50 \%$ and a standard deviation of $0.1 S_{0}$. Additionally, the excitation signal is kept constant for 200 seconds at the end of this aleatory excitation signal, so that the limit cycles can be mitigated by $C_{p}$. Figure 6.2 presents an example of excitation signal for $S_{0}=24$ and the respective desired stem position $x_{d}$. Notice that this excitation signal forces many movement reversals, which is the worst case that has to be compensated by $C_{p}$. 


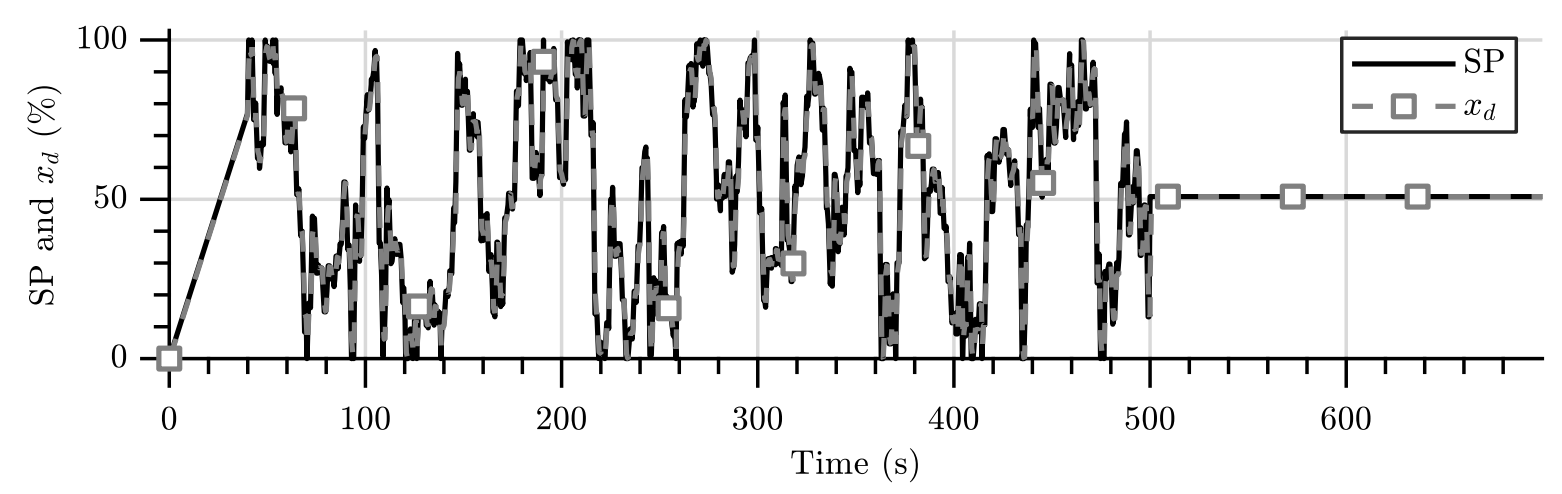

Figure 6.2 - Example of the excitation signal used to obtain the optimal parameters of $C_{p}$.

Source: from Author.

Another important characteristic regarding the AIC framework is to select a cost function. Even though Figure 6.1 shows that only the difference between the simulated and the desired stem positions is used to tune $C_{p}$, in practice this is not desirable, since it may lead to extremely high control efforts or an attempt to vigorously correct any measurement noise that the sensors may present. Therefore, the AIC cost function used in this work encompass the control effort as well, which is usual for many optimal control techniques:

$$
J_{C}\left(\theta_{p}\right)=\sum_{n=1}^{n_{f}} g_{x}\left(\hat{x}\left(n, \theta_{p}\right)-x_{d}[n]\right)^{2}+g_{u}(\hat{\mathrm{OP}}[n-1]-\hat{\mathrm{OP}}[n])^{2}+\operatorname{pen}\left(\theta_{p}\right)
$$

where $\theta_{p}$ is the $C_{p}$ parameter vector, $g_{x}$ is the stem position deviation gain, $g_{u}$ is the controller output difference gain and pen $\left(\theta_{p}\right)$ is a function used to force the optimization to be inside a predefined search space, that is implemented as presented in Chapter 4 . The values of $g_{x}$ and $g_{u}$ were selected empirically as 1 and 0.1 , respectively, which tends to result in a controller tuning that follows the desired dynamics regardless the control effort. The optimal controller tuning, $\theta_{p}{ }^{*}$, is calculated as:

$$
\theta_{p}^{*}=\underset{\theta_{p}}{\arg \min } J_{C}\left(\theta_{p}\right)
$$

The optimization algorithm used to obtain the minimum of $J_{C}\left(\theta_{p}\right)$ is the same used to estimate the friction parameters in Chapter 4, the GDES.

Additionally, the stem position measurement noise is simulated in the AIC framework as an additive Gaussian random variable with zero mean and a variance that results in a $\mathrm{SNR}$ of $25 \mathrm{~dB}$.

The controller sampling time is potentially different from the integration time of the valve model, therefore, the AIC framework has to account for this difference, by using a zero order hold in the $C_{p}$ output. In this work, the controller sampling time is $20 \mathrm{~ms}$, as it is not possible to obtain lower values with the current hardware configuration of the FPP. 


\subsection{A First Test with PID control}

The PID controller is defined as:

$$
\begin{aligned}
P_{C_{p}}[n] & =K_{p} \hat{e}[n] \\
I_{C_{p}}[n] & =K_{i} \frac{T_{s_{C_{p}}}}{2}(\hat{e}[n]+\hat{e}[n-1])+I_{C_{p}}[n-1] \\
D_{C_{p}}[n] & =\frac{K_{d}(\hat{x}[n]-\hat{x}[n-1])+\tau_{d f} D_{C_{p}}[n-1]}{\tau_{d f}+T_{s_{C_{p}}}} \\
\hat{\mathrm{OP}}[n] & =P_{C_{p}}[n]+I_{C_{p}}[n]+D_{C_{p}}[n],
\end{aligned}
$$

where $\hat{e}[n]=\mathrm{ES}[n]-\hat{x}[n], \mathrm{ES}$ is the AIC framework excitation signal (or SP in the practical applications), $T_{s_{C_{p}}}=20 \mathrm{~ms}$ is the controller sampling time and a back calculation technique is used to ensure that the integral action, $I_{C_{p}}[n]$, stops integrating errors outside the actuator range. The PID controller parameter search space is presented in Table 6.1.

Table 6.1 - PID controller parameter search space.

\begin{tabular}{ccc} 
Parameter & $u b_{d}$ & $l b_{d}$ \\
\hline$K_{p}$ & 50 & 0.1 \\
$K_{i}$ & $10 / \hat{\tau}_{\mathrm{I} / \mathrm{P}}$ & 0 \\
$K_{d}$ & $10 \hat{\tau}_{\mathrm{I} / \mathrm{P}}$ & 0 \\
$\tau_{d f}$ & $100 \hat{\tau}_{\mathrm{I} / \mathrm{P}}$ & 0 \\
\hline
\end{tabular}

This test is performed using the graphite gasket valve with estimated valve model as presented in Chapters 4 and 5. Unlike the algorithms to estimate the parameters of valve and friction models, the I/P model time constant is very important when tuning the valve position controller, as it may be the system dominant time constant. Thus, in this test, the controllers are tuned using open and closed loop estimated Kano, Karnopp and GMS valve models. Even though the closed loop estimated valve model has only one numerical value for the I/P model time constant, the open loop estimated models have the possibility to use the step or $\operatorname{ramp}\left(\hat{\tau}_{\mathrm{I} / \mathrm{P}_{s}}\right.$ and $\left.\hat{\tau}_{\mathrm{I} / \mathrm{P}_{r}}\right) \mathrm{I} / \mathrm{P}$ model estimated time constants. Table 6.2 presents the optimal PID parameter tuning for each estimated valve model. Notice that the optimal tuning varies greatly, depending on the estimated valve model and the I/P model time constant used in the AIC framework. 
Table 6.2 - PID tunings for each estimated model used in the AIC framework.

\begin{tabular}{lrrrr} 
Model & $K_{p}{ }^{*}$ & \multicolumn{1}{c}{$K_{i}{ }^{*}$} & $K_{d}{ }^{*}$ & $\tau_{d f}{ }^{*}$ \\
\hline Kano OL, $\hat{\tau}_{\mathrm{I} / \mathrm{P}_{r}}$ & 5.9 & 11.3 & 4.2 & 2.9 \\
Kano OL, $\hat{\tau}_{\mathrm{I} / \mathrm{P}_{s}}$ & 5.8 & 9.4 & 9.1 & 3.1 \\
Kano CL & 4.0 & 14.2 & 2.3 & 1.9 \\
Karnopp OL, $\hat{\tau}_{\mathrm{I} / \mathrm{P}_{r}}$ & 8.8 & 22.7 & 4.2 & 1.3 \\
Karnopp OL, $\hat{\tau}_{\mathrm{I} / \mathrm{P}_{s}}$ & 6.9 & 8.7 & 9.3 & 3.4 \\
Karnopp CL & 7.0 & 11.9 & 0.6 & 0.1 \\
GMS OL, $\hat{\tau}_{\mathrm{I} / \mathrm{P}_{r}}$ & 7.1 & 15.5 & 4.2 & 2.5 \\
GMS OL, $\hat{\tau}_{\mathrm{I} / \mathrm{P}_{s}}$ & 6.3 & 7.0 & 8.8 & 3.9 \\
GMS CL 5.2 & 16.3 & 2.3 & 1.3 \\
\hline \multicolumn{4}{c}{ Source: from Author. }
\end{tabular}

OL: model with open loop estimated parameters and CL: model with closed loop estimated parameters.

The hydraulic circuit controller, $C_{H}$, is a PI, with proportional gain $K_{H}=0.564$ and integral gain $K_{i H}=0.31$, calculated as presented in Section 5.5.

For each PID tuning, three tests are performed: a servo, a regulatory and an aleatory. The servo test consists in maintaining $\mathrm{SP}_{Q}$ constant at a given value and modifying it using a step function at $300 \mathrm{~s}$ and $600 \mathrm{~s}$. In the regulatory test, the $\mathrm{SP}_{Q}$ is kept constant during the test, but the perturbation valve (FY-13) position is modified at $300 \mathrm{~s}$ and $600 \mathrm{~s}$, using a step function. In the aleatory test, $\mathrm{SP}_{Q}$ is modified using a stream of aleatory velocity ramps, similar to the excitation signal used to obtain the optimal $C_{p}$ parameters, and it is maintained constant at the last 300 seconds of the test. Some examples are presented in Figures 6.3 to 6.5 .

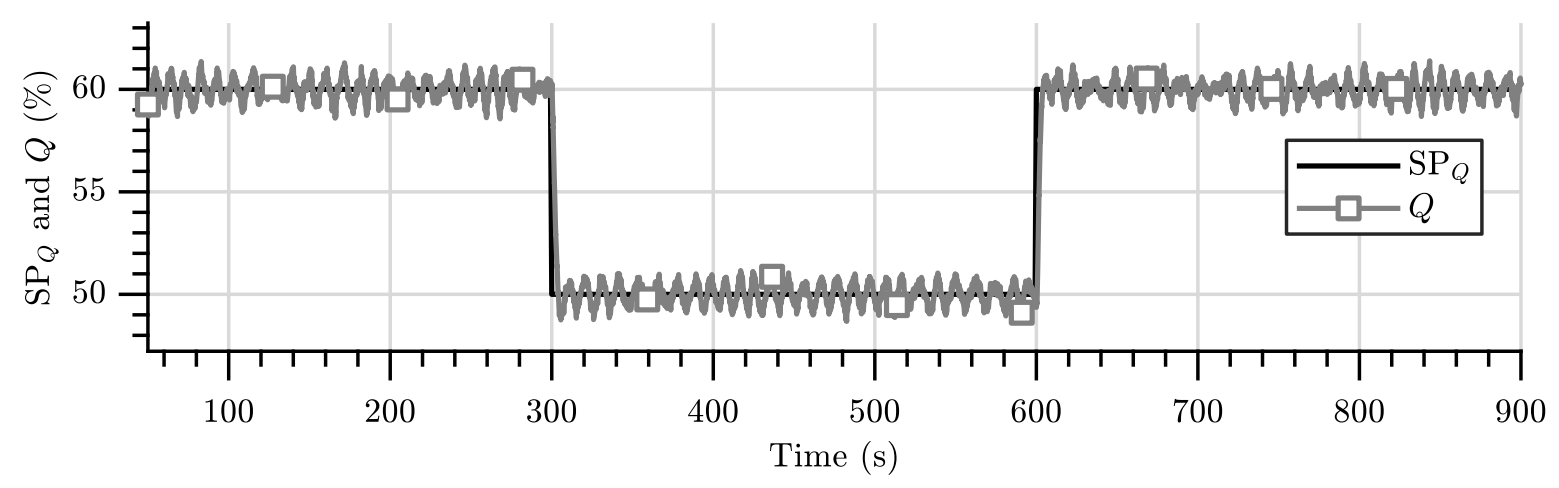

Figure 6.3 - Servo test example, using the PID controller tuned with the Kano OL and $\tau_{\mathrm{I} / \mathrm{P}_{r}}$ estimated model.

Source: from Author. 


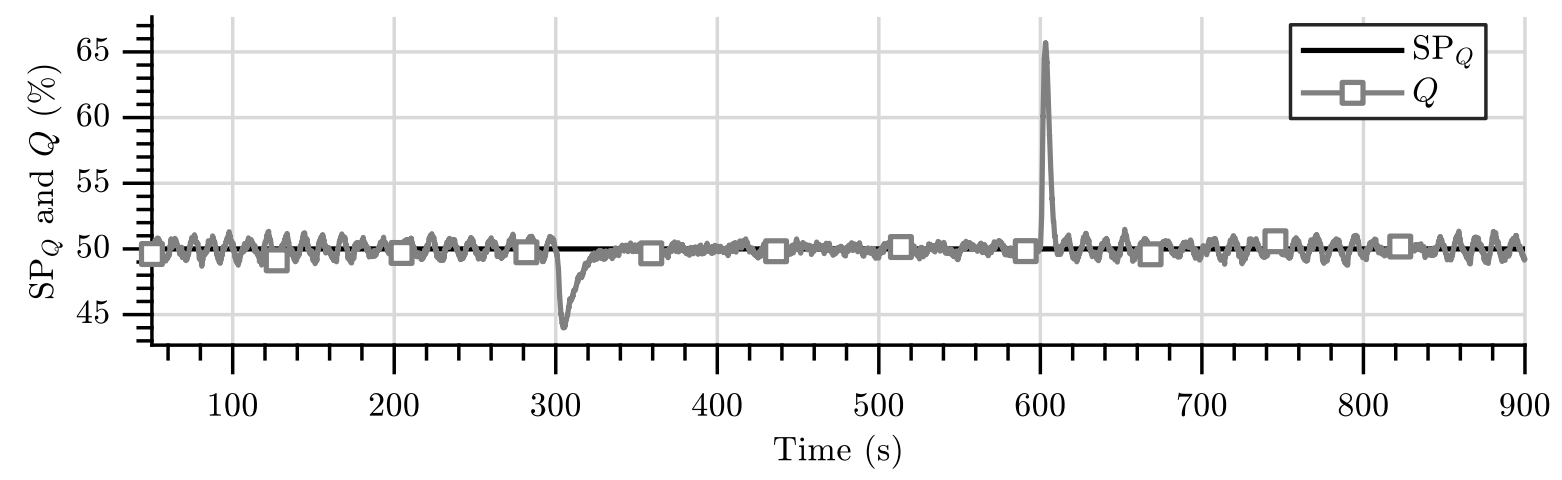

Figure 6.4 - Regulatory test example, using the PID controller tuned with the Karnopp OL and $\tau_{\mathrm{I} / \mathrm{P}_{s}}$ estimated model.

Source: from Author.

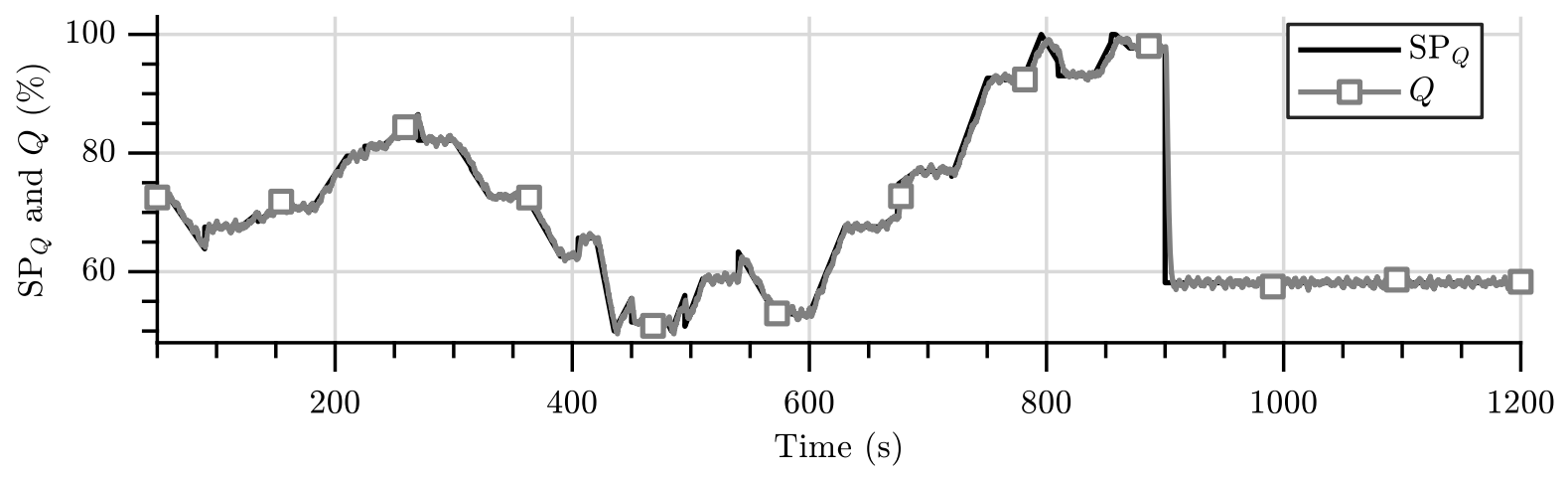

Figure 6.5 - Aleatory test example, using the PID controller tuned with the GMS CL estimated model.

Source: from Author.

Note that in every test it is possible to observe that the hydraulic circuit flow rate $(Q)$ presents limit cycle. When using cascaded control loops, which is the case when using a digital positioner, the system tends to be more oscillatory in presence of high friction, as can be seen in the works of Hidalgo and Garcia (2017) and Baeza and Garcia (2018). This happens due to an accumulation of errors between the cascaded controller integrators, which makes it very difficult to eliminate the limit cycles in such systems by tuning $C_{p}$ only.

For comparison purposes, a first order plus dead time model is estimated for the valve, using a step excitation signal, resulting in the following transfer function, in the Laplace domain:

$$
\frac{\mathrm{OP}(s)}{x(s)}=\frac{0.8243}{1.073 s+1} \mathrm{e}^{-0.56 s} .
$$

Applying the DS method to obtain a closed loop time constant $50 \%$ faster than the model time constant, a PI controller is calculated as $K_{p}=1.2$ and $K_{i}=1.1$. 
To compare the tuning performance, it is possible to calculate the integral absolute flow rate error $\left(\operatorname{iae}_{Q}\right)$ and stem position error $\left(\right.$ iae $\left._{x}\right)$ indexes, that are defined as:

$$
\begin{aligned}
\operatorname{iae}_{Q} & =\sum_{n=0}^{n_{f}}\left|\operatorname{SP}_{Q}[n]-Q[n]\right| \\
\operatorname{iae}_{x} & =\sum_{n=0}^{n_{f}}|\operatorname{SP}[n]-x[n]|
\end{aligned}
$$

Grouping the experiment performance indexes into types of estimated models used in the AIC framework, it is possible to create a box plot, as presented in Figure 6.6. It is possible to verify that it is more likely to obtain better results when the open loop estimated valve model with $\hat{\tau}_{\mathrm{I} / \mathrm{P}_{r}}$ ortthe closed loop estimated valve model are used in the AIC framework. When $\hat{\tau}_{\mathrm{I} / \mathrm{P}_{s}}$ is used in the open loop estimated valve model, the tunings tend to be worst. The DS tuned PI controller performances are not shown, as its lower indexes are $\operatorname{iae}_{Q}=110421$ and iae $_{x}=107265$, much higher than any PID tuned using the AIC framework.

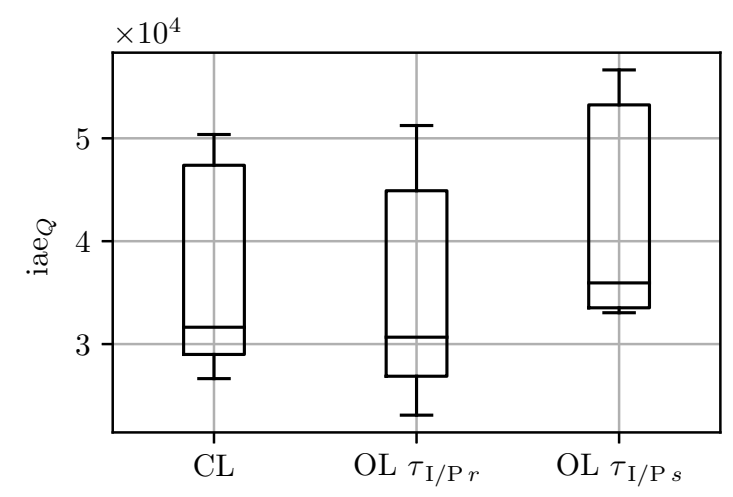

(a) Comparison of the $\operatorname{iae}_{Q}$.

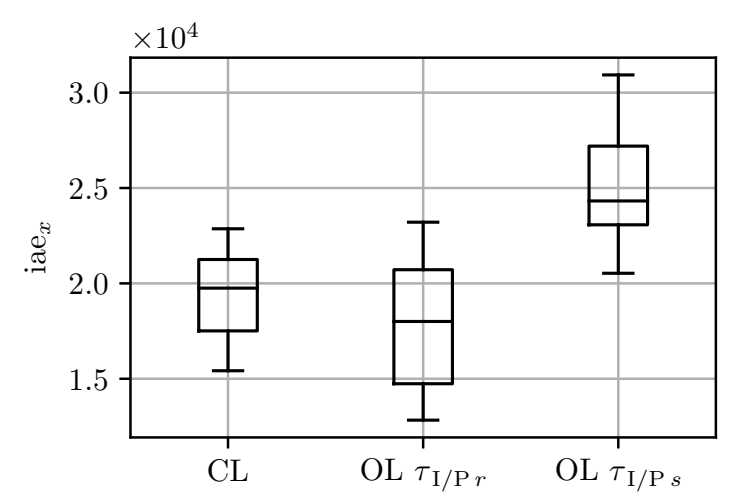

(b) Comparison of the iae .

Figure 6.6 - Comparison of the tuning performance of the AIC method in the initial PID test, grouping data by model estimation type.

Source: from Author.

The comparison of performance between the estimated valve models used in the AIC framework are presented in Figures 6.7 to 6.9, where the controller output standard deviation index, $\operatorname{std}(\mathrm{OP})$, is introduced, which is calculated over periods of the experiment in which the $\mathrm{SP}_{Q}$ is maintained stable, therefore it indicates whether the control loop has a limit cycle, as this index should be close to zero when $\mathrm{SP}_{Q}$ is kept constant. It is possible to verify that the PID tuning has the best overall performance when open loop estimated Karnopp model is used together with $\hat{\tau}_{\mathrm{I} / \mathrm{P}_{r}}$, in the AIC framework. The GMS model, however, holds the best indexes when the valve model parameters are estimated in closed loop. From the performance indexes, it is possible to verify that the GMS model is pretty consistent with the friction parameters estimated either in closed or open loop, provided that the $\hat{\tau}_{\mathrm{I} / \mathrm{P}_{r}}$ time constant is used in the open loop valve model. 


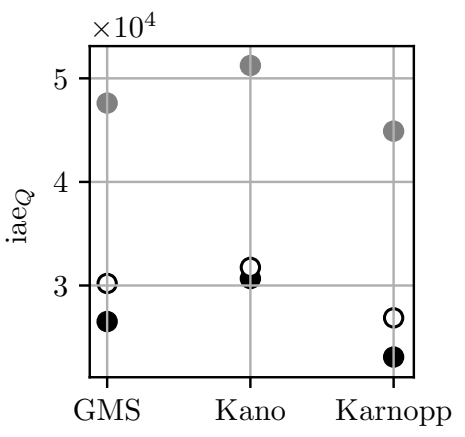

(a) Open loop with $\tau_{\mathrm{I} / \mathrm{P}_{r}}$.

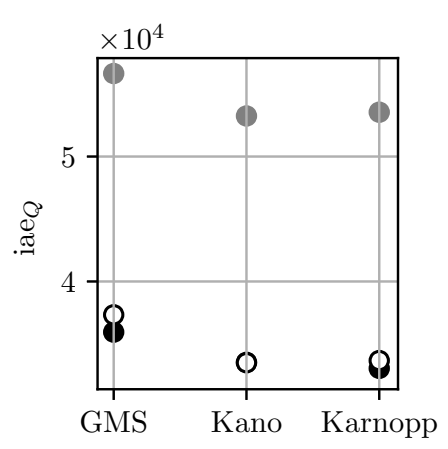

(b) Open loop with $\tau_{\mathrm{I} / \mathrm{P}_{s}}$.

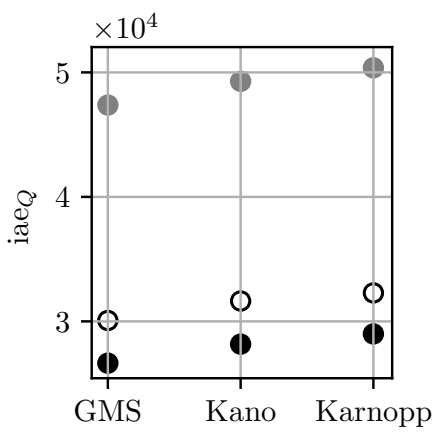

(c) Closed loop.

Figure 6.7 - Comparison of iae $_{Q}$, among the estimated friction models. Legend: regulatory (white), servo (black) and aleatory (gray) experiments. Source: from Author.

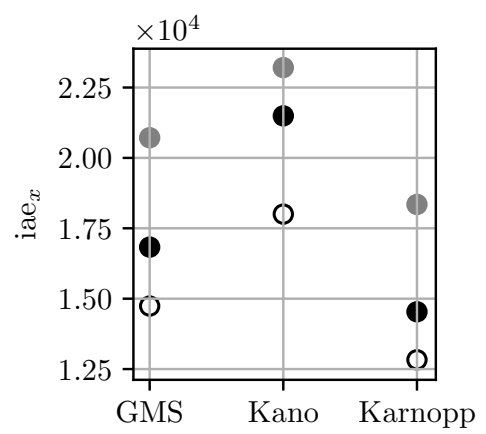

(a) Open loop with $\tau_{\mathrm{I} / \mathrm{P}_{r}}$.

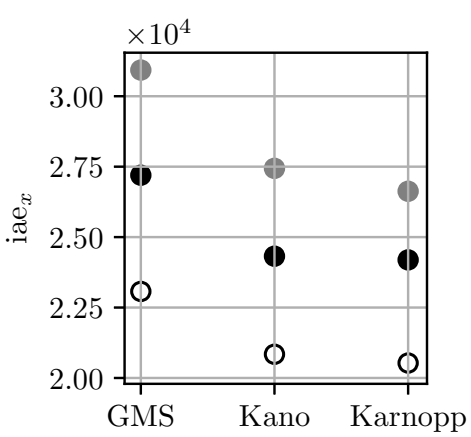

(b) Open loop with $\tau_{\mathrm{I} / \mathrm{P}_{s}}$.

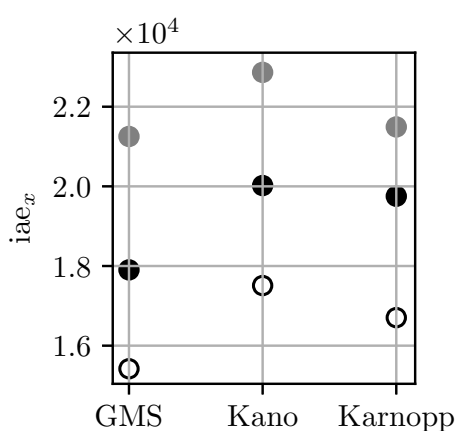

(c) Closed loop.

Figure 6.8 - Comparison of iae $_{x}$, among the estimated friction models.

Legend: regulatory (white), servo (black) and aleatory (gray) experiments.

Source: from Author.

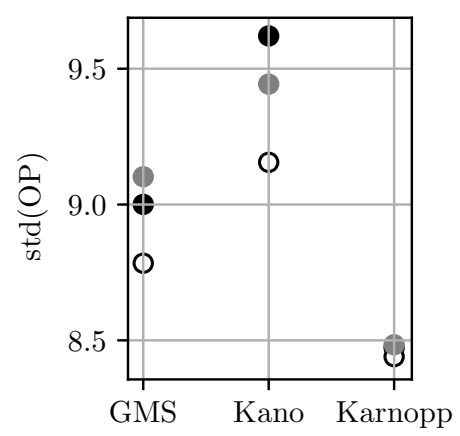

(a) Open loop with $\tau_{\mathrm{I} / \mathrm{P}_{r}}$.

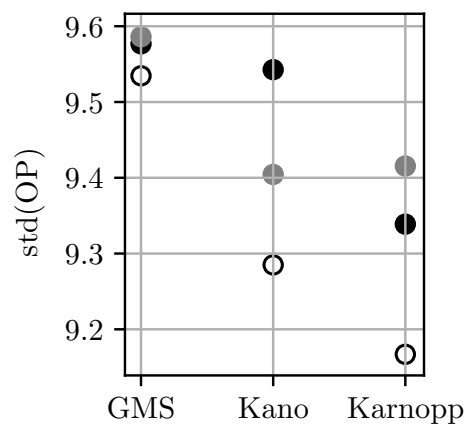

(b) Open loop with $\tau_{\mathrm{I} / \mathrm{P}_{s}}$.

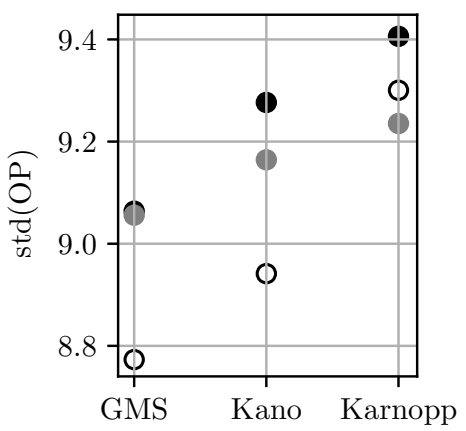

(c) Closed loop.

Figure 6.9 - Comparison of std (OP), among the estimated friction models. Legend: regulatory (white), servo (black) and aleatory (gray) experiments. Source: from Author.

For a time domain performance comparison, an extract of the aleatory experiment 
is presented in Figures 6.10 and 6.11. Note that even though the numerical performance indexes indicate differences among the tunings, the time domain comparison presents very similar responses, with a few visual differences.

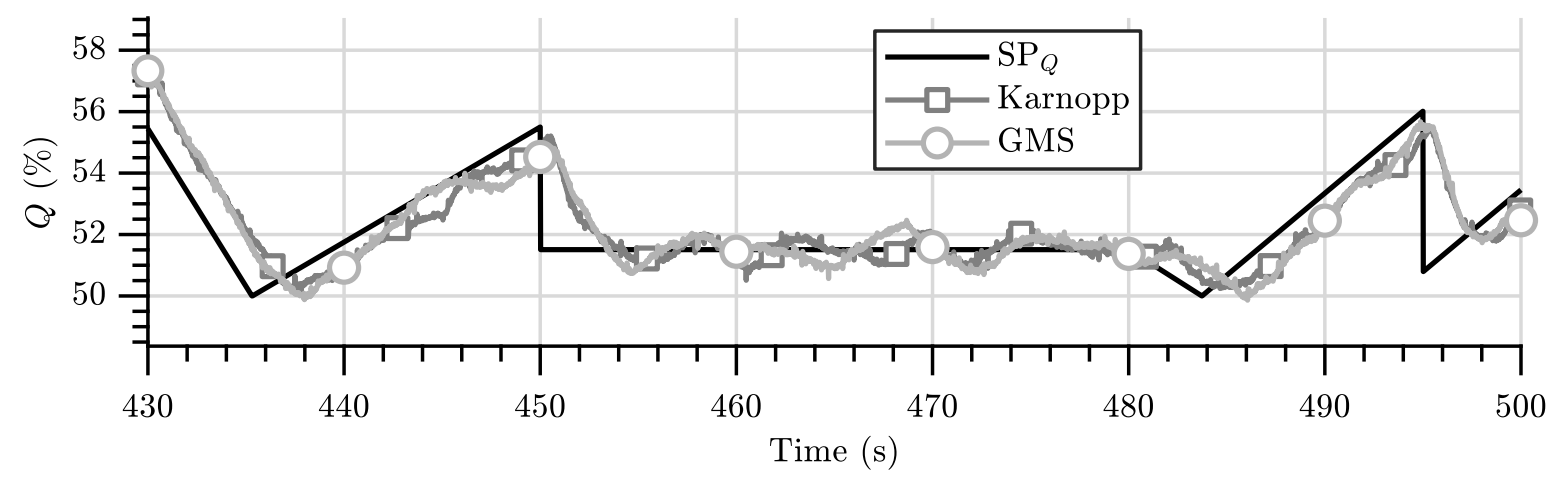

Figure 6.10 - Aleatory experiment extract using PID tuned with open loop estimated Karnopp and GMS models in the AIC framework (with $\hat{\tau}_{\mathrm{I} / \mathrm{P}_{r}}$ as the I/P model time constant).

Source: from Author.

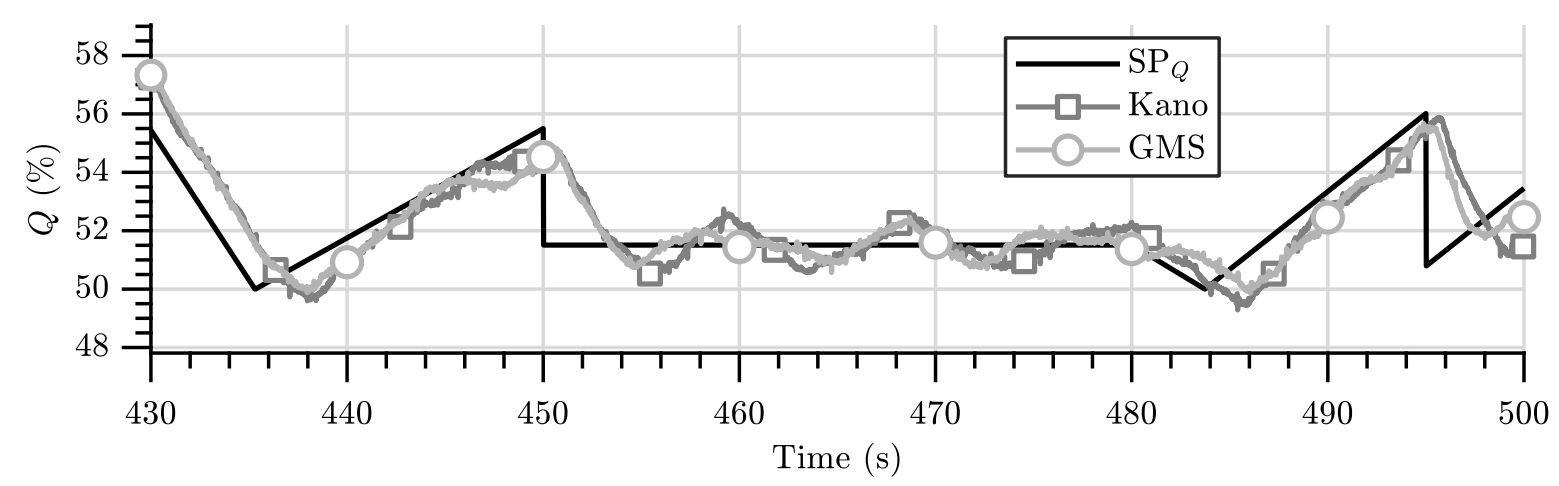

Figure 6.11 - Aleatory experiment extract using PID tuned with open loop estimated Kano and GMS models in the AIC framework (with $\hat{\tau}_{\mathrm{I} / \mathrm{P}_{r}}$ as the I/P model time constant).

Source: from Author.

\subsection{A Robustness Test}

As the I/P model time constant seems to play a major role regarding the controller performance, some tests are carried out to verify if the proposed AIC framework is able to deliver good controllers even if $\tau_{\mathrm{I} / \mathrm{P}_{s}}$ is used for the I/P model time constant, and yet it is assumed that this parameter is estimated with a $+100 \%$ error.

In this test, besides the PID controller, some other usual friction compensation techniques are used as well, such as the control freezing, the knocker, the constant reinforcement and the fuzzy PID controller, which are described in the following subsections. 
The DS calculated PI controller is adjusted accordingly, to simulate a $+100 \%$ error in the valve transfer function time constant estimation, resulting in: $K_{p}=1.59$ and $K_{i}=0.741$.

\subsubsection{The Control Freezing Algorithm (CF)}

In this method, the controller output is kept equal to the last value if the absolute error $(|\hat{e}[n]|)$ is lower than $e_{l}$ for $t_{e_{l}}$ seconds and the controller starts to update its output again if the absolute error is higher than $e_{h}$ for $t_{e_{h}}$ seconds (SILVA; GARCIA, 2014). In this work, the algorithm is slightly modified, as only the integral action and the friction compensator algorithm of the controller are disabled, instead of freezing the controller output. This method is used together with a regular PID controller and it is intended to eliminate the control loop limit cycles.

These parameters are suggested to be tuned as $e_{l}=e_{h}=0.5, t_{e_{l}}=10 \mathrm{~s}$ and $t_{e_{h}}=2 \mathrm{~s}$ (SILVA; GARCIA, 2014). However, after some empirical tests, it was verified that for the FPP, the best results are obtained by using $e_{l}=e_{h}=1$.

The search space of the CF algorithm parameters, used in the AIC framework, is defined in Table 6.3. The negative lower bound of the $e_{l}$ and $e_{h}$ parameters is used to allow searching for tunings in which the CF algorithm is disabled.

Table 6.3 - CF method parameter search space.

\begin{tabular}{ccc} 
Parameter & $u b_{d}$ & $l b_{d}$ \\
\hline$e_{l}$ & 10 & -1 \\
$e_{h}$ & 10 & -1 \\
$t_{e_{l}}$ & 20 & 0 \\
$t_{e_{h}}$ & 20 & 0 \\
\hline
\end{tabular}

\subsubsection{The Knocker Compensator}

This compensator idea is to add or subtract a high frequency signal to the PID output, so that the friction force can be overcome without influencing significantly the control loop. The Knocker compensator signal has a period, $t_{k_{p}}$, a duty cycle, $d_{k_{p}}$ and an amplitude, $a_{k n}$. The suggested values for these parameters are $t_{k_{p}}=5 T_{s_{C_{p}}} \mathrm{~s}, d_{k_{p}}=0.4$ and $a_{k n}=S_{0} / 2$ (SILVA; GARCIA, 2014). In this work, the Knocker compensator is used combined with the PID and the CF algorithm.

The selection between adding or subtracting the Knocker signal to the controller output is determined by the equation:

$$
\operatorname{sig}_{k}=\operatorname{sgn}\left(\hat{\mathrm{OP}}[n]-\hat{\mathrm{OP}}\left[n-t_{k_{p}} / T_{s_{C_{p}}}\right]\right),
$$

such that if $\operatorname{sig}_{k}$ is positive, then the compensator signal is added to the PID output and if $\operatorname{sig}_{k}$ is negative, the compensator signal is subtracted, instead. 
The search space of the Knocker compensator, used in the AIC framework, is presented in Table 6.4 .

Table 6.4 - CF method parameter search space.

\begin{tabular}{ccc} 
Parameter & $u b_{d}$ & $l b_{d}$ \\
\hline$t_{k_{p}}$ & $\hat{\tau}_{\mathrm{I} / \mathrm{P}}$ & 0 \\
$d_{k_{p}}$ & 1 & 0 \\
$a_{k n}$ & 100 & 0 \\
\hline
\end{tabular}

\subsubsection{The Constant Reinforcement Compensator (CR)}

This compensator is simpler than the Knocker, as it simply adds or subtracts a constant from the PID controller output, such that the valve deadband can be neglected when tuning the PID controller. The only parameter of this algorithm is a constant, which is usually suggested as $a_{C R}=S_{0} / 2$ (SILVA; GARCIA, 2014). Similarly to the Knocker, this compensator is used together with the PID and the CF algorithm.

The addition or subtraction of the CR signal constant to the PID output is calculated as:

$$
\operatorname{sig}_{C R}=\operatorname{sgn}\left(\hat{\mathrm{OP}}[n]-\hat{\mathrm{OP}}\left[n-1 / T_{s_{C_{p}}}\right]\right),
$$

where $a_{C R}$ is added to the PID output if $\operatorname{sig}_{C R}$ is positive, and subtracted if $\operatorname{sig}_{C R}$ is negative.

The search space of this compensator parameter is: $a_{C R} \in[0,100]$.

\subsubsection{The Fuzzy PID Controller}

This method idea is to have a PID controller with an integral gain that varies using fuzzy logic, as developed by Mishra, Kumar and Rana (2014). Since the limit cycles are generated by the integral action of the controllers, then the fuzzy logic may mitigate the limit cycle amplitude, by reducing the integral gain when the control loop error is low. This controller has two membership functions, to determine whether the error and its rate of change are zero:

$$
\begin{aligned}
& m_{e z}(\hat{e})=\exp \left(-\frac{\hat{e}^{2}}{2 s d_{\hat{e}}^{2}}\right) \\
& m_{r z}(\dot{\hat{e}})=\exp \left(-\frac{\dot{\hat{e}}^{2}}{2 s d_{\hat{\hat{e}}}^{2}}\right) .
\end{aligned}
$$

The corresponding membership functions that determine whether the variables are nonzero are calculated as:

$$
\begin{gathered}
m_{e n z}(\hat{e})=1-m_{e z}(\hat{e}) \\
m_{r n z}(\dot{\hat{e}})=1-m_{r z}(\dot{\hat{e}})
\end{gathered}
$$


The integral gain is defined by the following rules:

Rule 1: If $\hat{e}$ is nonzero and $\dot{\hat{e}}$ is nonzero, then $K_{f i}=K_{i_{1}}$;

Rule 2: If $\hat{e}$ is zero and $\dot{\hat{e}}$ is nonzero, then $K_{f i}=K_{i_{2}}$;

Rule 3: If $\hat{e}$ is nonzero and $\dot{\hat{e}}$ is zero, then $K_{f i}=K_{i_{3}}$ and

Rule 4: If $\hat{e}$ is zero and $\dot{\hat{e}}$ is zero, then $K_{f i}=K_{i_{4}}$.

The crisp integral gain is calculated using a T-norm:

$$
K_{f i}=\frac{m_{e n z} m_{r n z} K_{i_{1}}+m_{e z} m_{r n z} K_{i_{2}}+m_{e n z} m_{r z} K_{i_{3}}+m_{e z} m_{r z} K_{i_{4}}}{m_{e n z} m_{r n z}+m_{e z} m_{r n z}+m_{e n z} m_{r z}+m_{e z} m_{r z}},
$$

and the PID output is calculated as presented in Eq. (6.5), but with an integral gain that varies $\left(K_{f i}\right)$.

The suggested parameters of this controller are $s d_{\hat{e}}=1, s d_{\hat{\hat{e}}}=0.15, K_{i_{1}}=K_{i}$, $K_{i_{2}}=K_{i_{4}}=K_{i} / 1000$ and $K_{i_{3}}=1.1 K_{i}$. To avoid noise differentiation, the error rate of change is calculated using a first order filter with $\hat{\tau}_{\mathrm{I} / \mathrm{P}}$ time constant.

The search space of the fuzzy PID compensator, used in the AIC framework, is presented in Table 6.5. Note that the derivative and proportional terms search space is the same presented in Table 6.1.

Table 6.5 - Fuzzy PID controller parameter search space.

\begin{tabular}{ccc} 
Parameter & $u b_{d}$ & $l b_{d}$ \\
\hline$s d_{\hat{e}}$ & 50 & 0 \\
$s d_{\hat{\hat{e}}}$ & 50 & 0 \\
$K_{i_{1}}, K_{i_{2}}, K_{i_{3}}$ and $K_{i_{4}}$ & $10 / \hat{\tau}_{\mathrm{I} / \mathrm{P}}$ & 0 \\
\hline
\end{tabular}

\subsubsection{Robustness Test Results}

For each controller, the AIC tuning is performed using each open loop estimated valve model, considering that the time constant of the I/P model is $2 \hat{\tau}_{\mathrm{I} / \mathrm{P}_{s}}$. The tunings for each model and control structure are presented in Tables 6.6 to 6.10. Regarding the PID with CF control structure, note that when using the Karnopp model for the tuning, $e_{h}$ is negative, which in practice disables the CF algorithm after $t_{e_{h}}$ seconds, whatever is the controller error. With respect to the PID with CF and Knocker controller structure, it is interesting to note that the AIC framework tunes the $a_{k n}$ parameter with a much lower value than what is suggested in the literature, which means that the Knocker compensation algorithm is almost disabled. In regard to the PID with CF and CR controller structure, when the Kano and Karnopp models are used, the proportional gain of the controllers is almost disabled and the CR amplitude is higher than what is suggested in the literature, 
whereas when the GMS model is used, the CR action is almost disabled. As for the Fuzzy PID control structure, the proportional and derivative parameters are similar to the tunings obtained for the PID control structure, but the integral terms differ substantially when a different estimated valve model is used in the AIC framework.

Table 6.6 - PID tunings in the robustness test.

\begin{tabular}{lcccc} 
Model & $K_{p}{ }^{*}$ & $K_{i}{ }^{*}$ & $K_{d}{ }^{*}$ & $\tau_{d f}{ }^{*}$ \\
\hline Kano & 3.5 & 1.8 & 23.4 & 13.5 \\
Karnopp & 3.5 & 2.0 & 23.5 & 12.8 \\
GMS & 3.3 & 1.9 & 23.5 & 12.8 \\
\hline \multicolumn{5}{c}{ Source: from Author. }
\end{tabular}

Table 6.7 - PID with CF tunings in the robustness test.

\begin{tabular}{lcccccrcc} 
Model & $K_{p}{ }^{*}$ & $K_{i}{ }^{*}$ & $K_{d}{ }^{*}$ & $\tau_{d f}{ }^{*}$ & $e_{l}{ }^{*}$ & $e_{h}{ }^{*}$ & $t_{e_{l}}{ }^{*}$ & $t_{e_{h}}{ }^{*}$ \\
\hline Kano & 4.1 & 3.0 & 23.4 & 11.1 & 9.7 & 0.2 & 1.8 & 1.0 \\
Karnopp & 3.2 & 2.5 & 23.1 & 15.1 & 7.7 & -0.2 & 1.4 & 1.1 \\
GMS & 5.3 & 3.8 & 22.8 & 12.3 & 2.6 & 2.4 & 0.2 & 1.9 \\
\hline \multicolumn{6}{c}{ Source: from Author. }
\end{tabular}

Table 6.8 - PID with CF and Knocker friction compensation tunings in the robustness test.

\begin{tabular}{lcrrrrrrrrrr} 
Model & $K_{p}{ }^{*}$ & $K_{i}{ }^{*}$ & $K_{d}{ }^{*}$ & $\tau_{d f}{ }^{*}$ & $e_{l}{ }^{*}$ & $e_{h}{ }^{*}$ & $t_{e_{l}}{ }^{*}$ & $t_{e_{h}}{ }^{*}$ & $t_{k_{p}}{ }^{*}$ & $d_{k_{p}}{ }^{*}$ & $a_{k n}{ }^{*}$ \\
\hline Kano & 3.0 & 1.1 & 3.3 & 9.4 & 3.1 & 1.8 & 0.002 & 0.1 & 1.7 & 0.4 & 4.9 \\
Karnopp & 5.8 & 4.2 & 21.2 & 11.9 & 9.4 & 1.1 & 2.0 & 2.2 & 1.0 & 0.01 & 1.0 \\
GMS & 5.0 & 4.2 & 23.1 & 14.3 & 2.2 & 2.5 & 0.08 & 2.2 & 0.2 & 0.5 & 1.7 \\
\hline \multicolumn{1}{c}{ Source: from Author. }
\end{tabular}

Table 6.9 - PID with CF and CR friction compensation tunings in the robustness test.

\begin{tabular}{lrrrrrrrrr} 
Model & $K_{p}{ }^{*}$ & $K_{i}{ }^{*}$ & $K_{d}{ }^{*}$ & $\tau_{d f}{ }^{*}$ & $e_{l}{ }^{*}$ & $e_{h}{ }^{*}$ & $t_{e_{l}}{ }^{*}$ & $t_{e_{h}}{ }^{*}$ & $a_{C R}{ }^{*}$ \\
\hline Kano & 0.1 & 0.4 & 1.8 & 101.8 & 0.9 & 0.8 & 0.09 & 0.2 & 22.9 \\
Karnopp & 0.4 & 0.4 & 1.1 & 37.0 & 2.9 & 0.8 & 9.9 & 0.3 & 19.6 \\
GMS & 5.44 & 4.2 & 23.1 & 11.6 & 3.2 & 3.2 & 0.5 & 2.0 & 0.001 \\
\hline \multicolumn{8}{c}{ Source: from Author. }
\end{tabular}

Table 6.10 - Fuzzy PID tunings in the robustness test.

\begin{tabular}{lccccccccr} 
Model & $K_{p}{ }^{*}$ & $K_{d}{ }^{*}$ & $\tau_{d f}{ }^{*}$ & $K_{i_{1}}{ }^{*}$ & $K_{i_{2}}{ }^{*}$ & $K_{i_{3}}{ }^{*}$ & $K_{i_{4}}{ }^{*}$ & $s d_{\hat{e}}{ }^{*}$ & $s d_{\hat{\hat{e}}}{ }^{*}$ \\
\hline Kano & 3.85 & 21.9 & 11.6 & 0.3 & 0.4 & 1.9 & 0.02 & 0.9 & 13.5 \\
Karnopp & 3.24 & 23.3 & 16.0 & 3.2 & 1.6 & 4.2 & 0.9 & 9.7 & 0.7 \\
GMS & 3.51 & 23.4 & 14.7 & 1.0 & 4.1 & 2.9 & 0.01 & 2.4 & 1.3 \\
\hline \multicolumn{8}{c}{ Source: from Author. }
\end{tabular}

The performed tests are the same ones presented in Section 6.2. Figure 6.12 shows the performance comparison, using box plot, grouping the results of the methods used to 
tune the controllers parameters. In general, the AIC tuning provides better performances than the DS method. However, there are two outliers, with excellent results, when using the DS method, that are given by the PID with CF and CR controller structure.

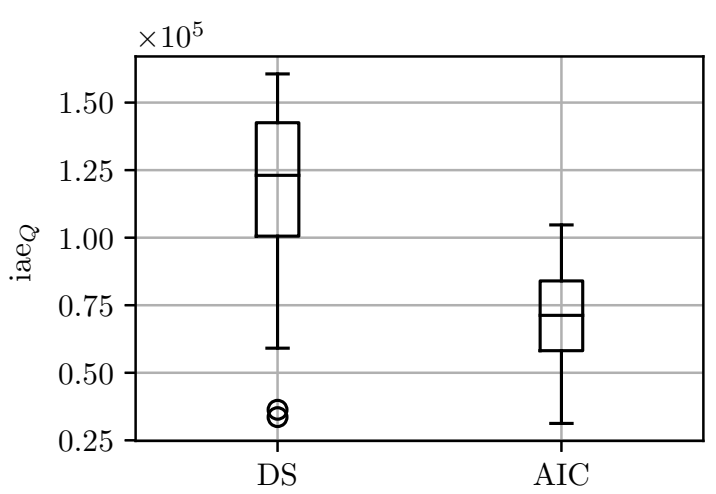

(a) Comparison of the $\operatorname{iae}_{Q}$.

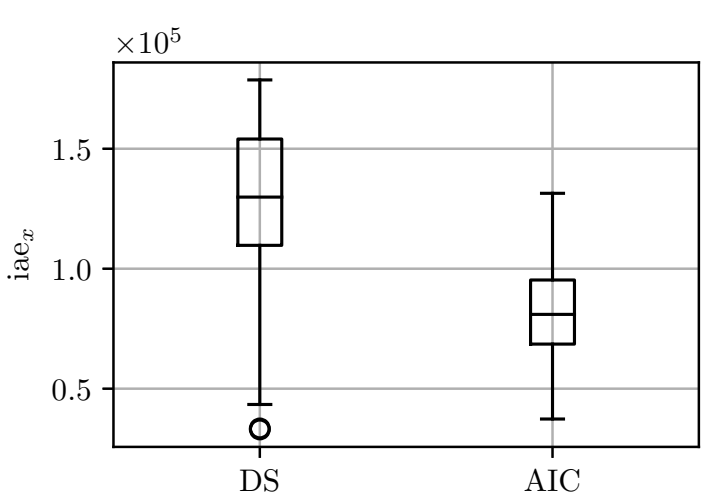

(b) Comparison of the iae ${ }_{x}$.

Figure 6.12 - Comparison of tuning performance using DS or AIC methods, in the robustness test.

Source: from Author.

A performance comparison among the estimated valve models used in the AIC framework is presented in Figure 6.13. The GMS model tends to present better performances regarding the $\mathrm{iae}_{Q}$ and $\operatorname{std}(\mathrm{OP})$ indexes, whereas the Kano and Karnopp models tend to perform better regarding iae $_{x}$. The Karnopp std(OP) index indicates that the tuned controllers using the Karnopp friction model are more likely to result in loops that are more oscillatory than with the other models.

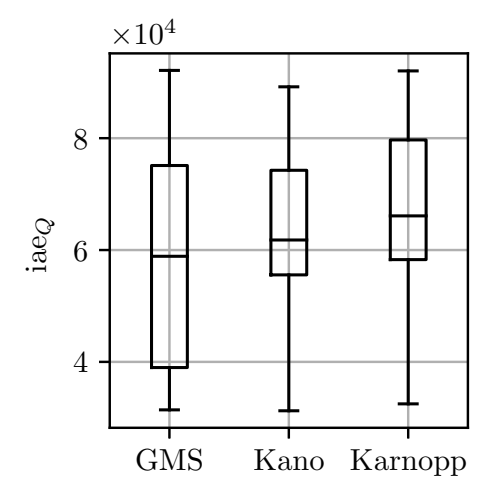

(a) Comparison of the iae $_{Q}$.

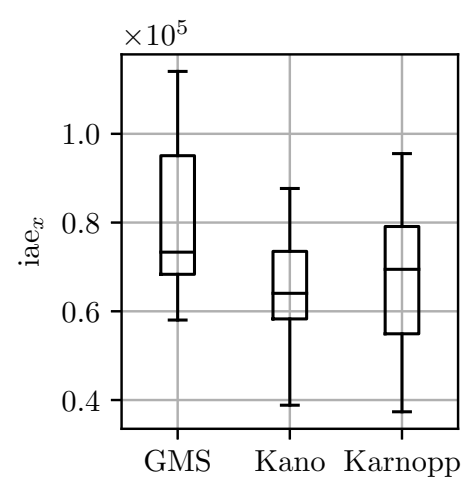

(b) Comparison of the iae $_{x}$.

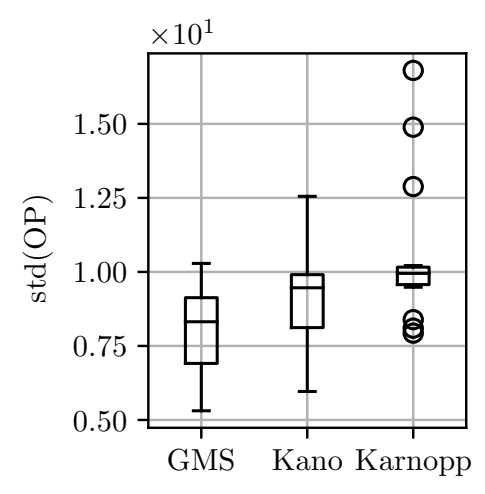

(c) Comparison of the std( $\mathrm{OP})$.

Figure 6.13 - Comparison of the AIC tuning performance when using different estimated models, in the robustness test.

Source: from Author.

The iae $_{Q}$ index comparison when using different friction models in the AIC framework, grouped by experiment type, is shown in Figure 6.14. The controller tunings are 
more likely to present better results when using the GMS friction model in AIC framework, however, the Kano and Karnopp result in very good tunings (shown as outliers), even better than when the GMS model is used, when the $C_{p}$ is the PID with CF and CR.

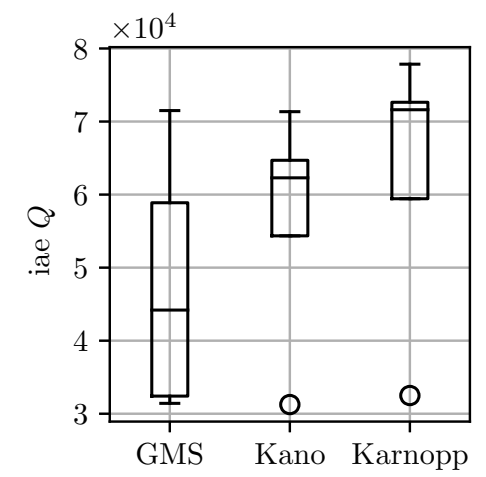

(a) Comparison of the $\operatorname{iae}_{Q}$, in the servo experiment.

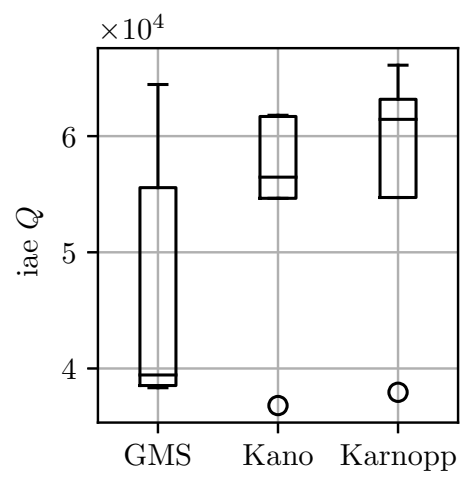

(b) Comparison of the $\operatorname{iae}_{Q}$, in the regulatory experiment.

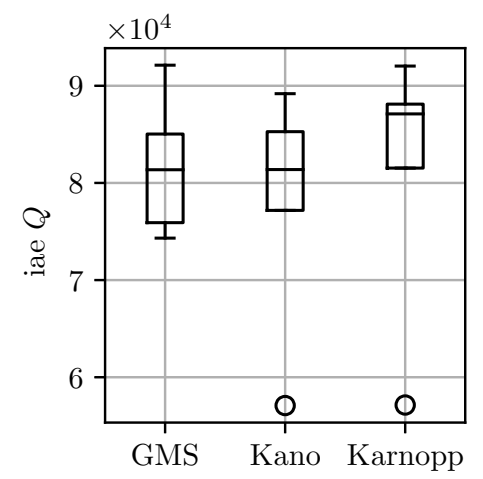

(c) Comparison of the $\operatorname{iae}_{Q}$, in the aleatory experiment.

Figure 6.14 - Comparison, for each experiment, of the friction models tuning performance, in the robustness test.

Source: from Author.

A performance comparison among the different control structures and compensators used in this test, for each estimated valve model used in the AIC framework, is presented in Figures 6.15 to 6.20. Once more, it is possible to verify that when the GMS model is used for tuning the controllers in the AIC framework, the results are more consistent, even though the best overall performances are from the Kano and Karnopp models, using the PID with CF and CR friction compensation. When the Karnopp model is used, it results in the highest $\operatorname{std}(\mathrm{OP})$ indexes. In general, the PID with $\mathrm{CF}$ and $\mathrm{CR}$ controller presents very good performance indexes.

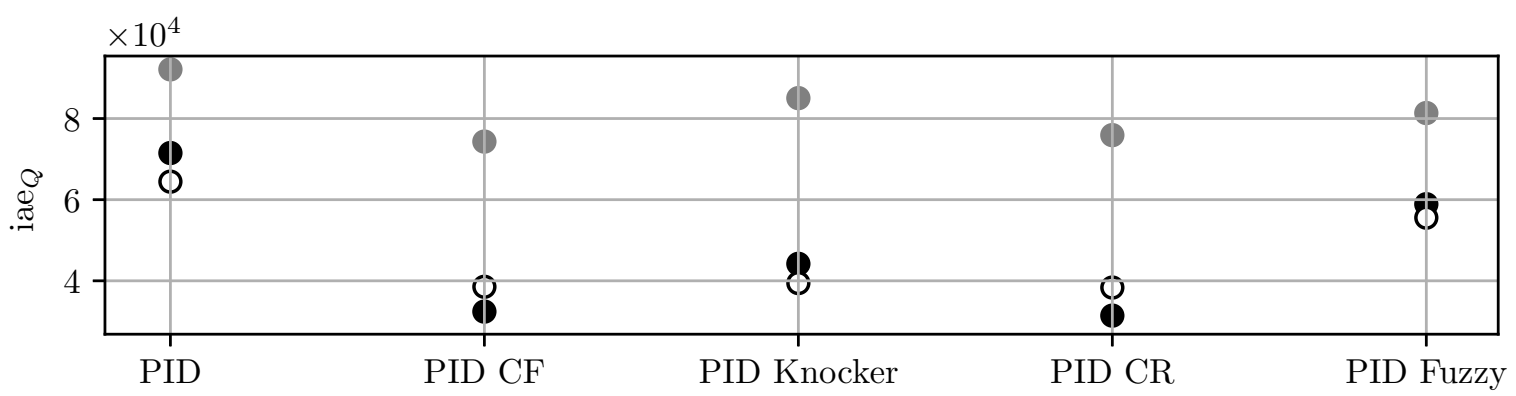

Figure 6.15 - Comparison of the iae $_{Q}$ index, using the GMS model in the AIC framework, for each controller, in the robustness test.

Legend: regulatory (white), servo (black) and aleatory (gray) experiments.

Source: from Author. 


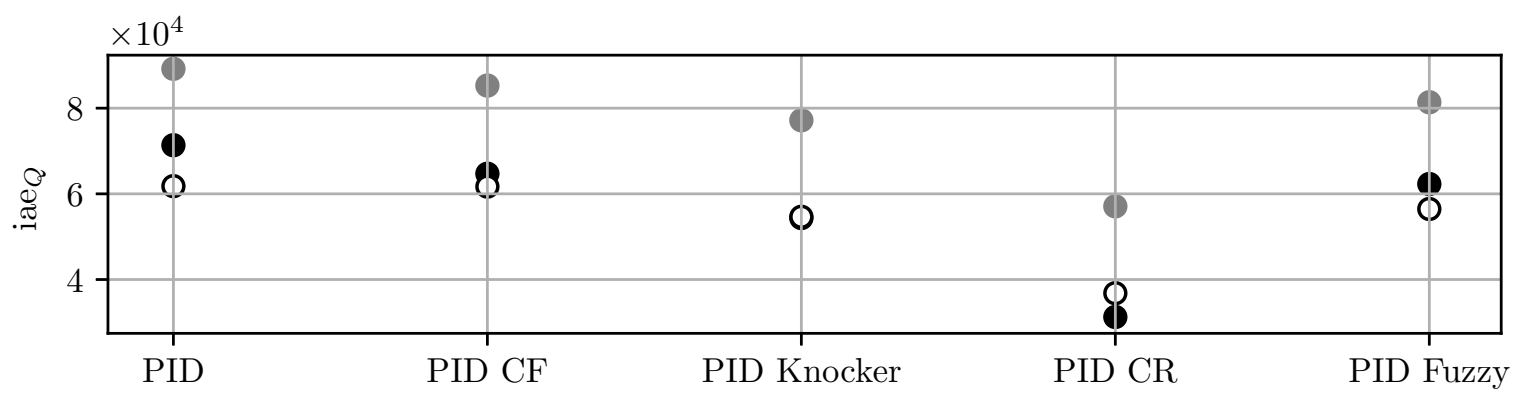

Figure 6.16 - Comparison of the iae $_{Q}$ index, using the Kano model in the AIC framework, for each controller, in the robustness test.

Legend: regulatory (white), servo (black) and aleatory (gray) experiments.

Source: from Author.

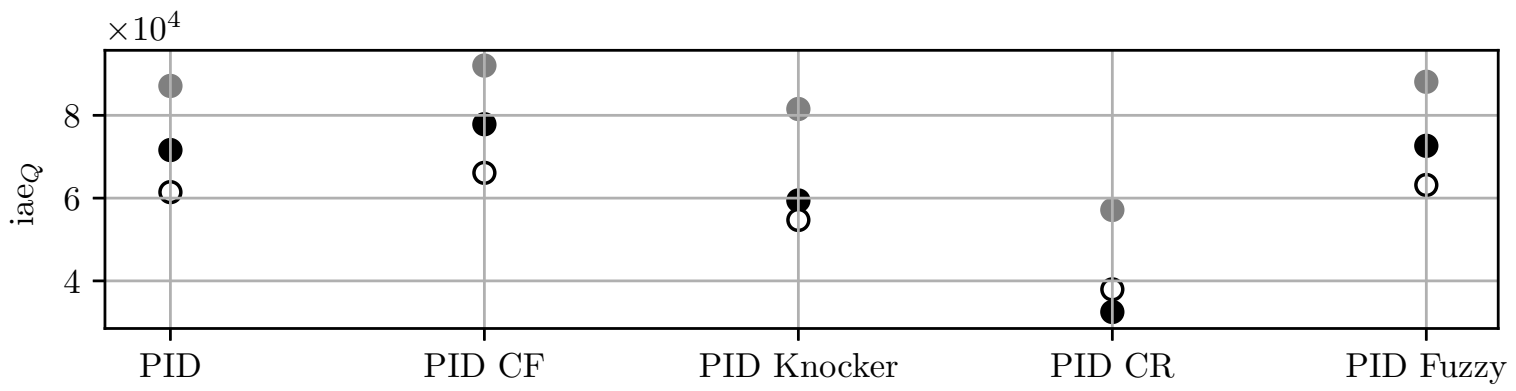

Figure 6.17 - Comparison of the iae $_{Q}$ index, using the Karnopp model in the AIC framework, for each controller, in the robustness test.

Legend: regulatory (white), servo (black) and aleatory (gray) experiments.

Source: from Author.

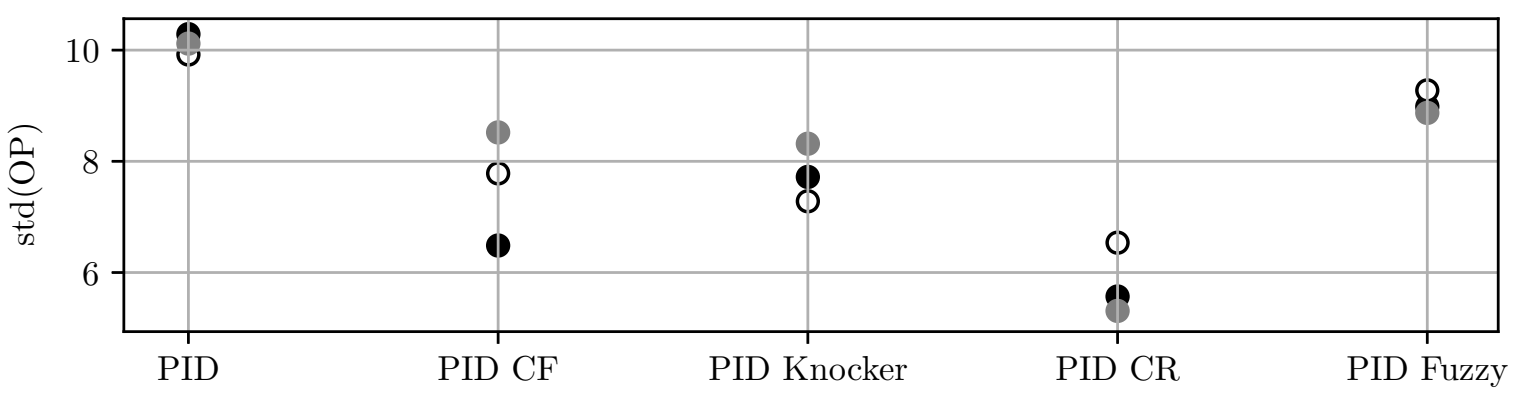

Figure 6.18 - Comparison of the std(OP) index, using the GMS model in the AIC framework, for each controller, in the robustness test.

Legend: regulatory (white), servo (black) and aleatory (gray) experiments.

Source: from Author. 


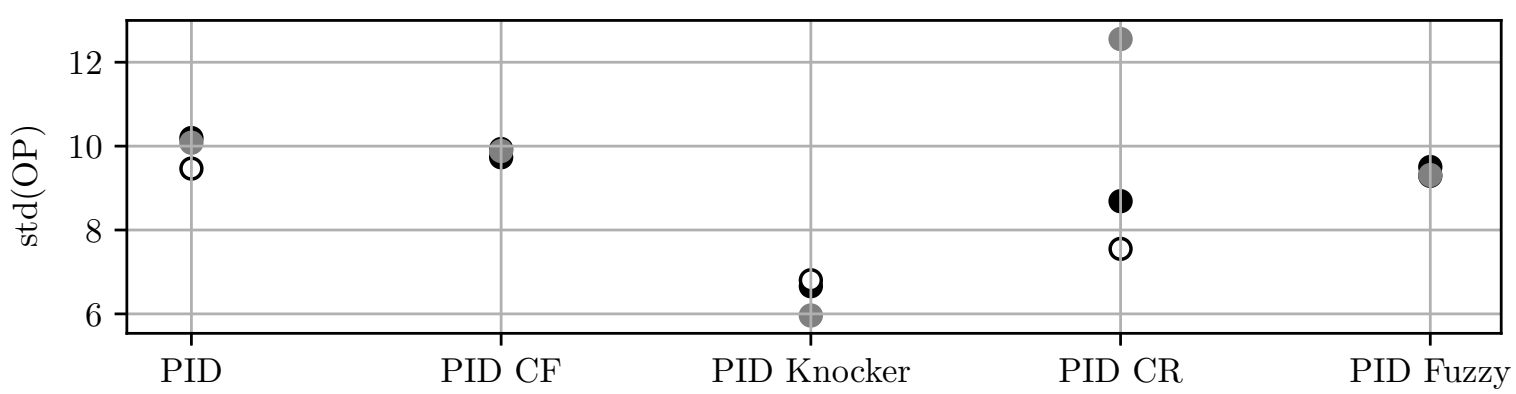

Figure 6.19 - Comparison of the std(OP) index, using the Kano model in the AIC framework, for each controller, in the robustness test.

Legend: regulatory (white), servo (black) and aleatory (gray) experiments.

Source: from Author.

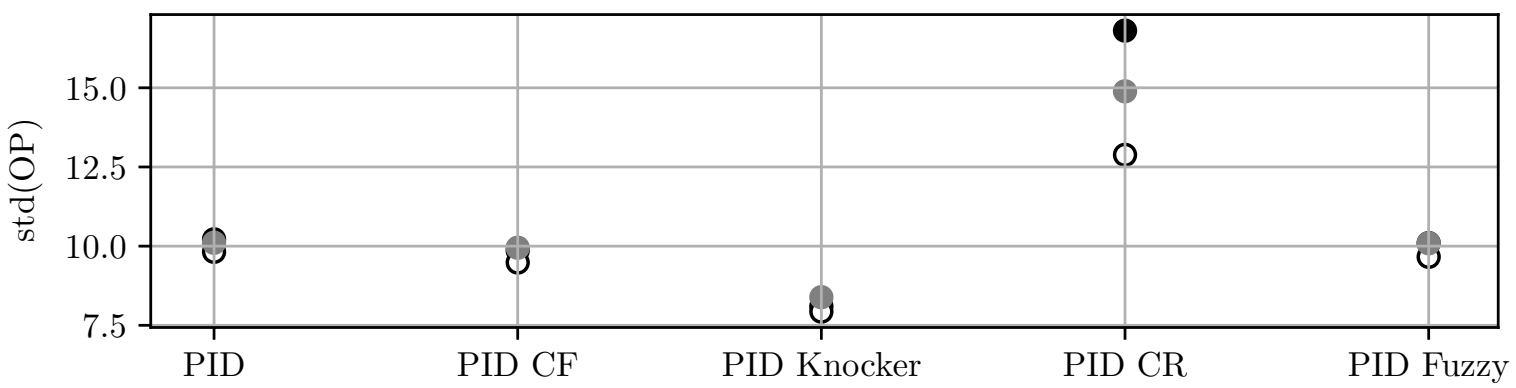

Figure 6.20 - Comparison of the std(OP) index, using the Karnopp model in the AIC framework, for each controller, in the robustness test.

Legend: regulatory (white), servo (black) and aleatory (gray) experiments.

Source: from Author.

A comparison between the AIC and DS tuning methods are presented in Figures 6.21 to 6.23 . In general, the DS method produces tunings that perform poorer when compared to the AIC tuning method, however, the PID with CF and CR is an exception, as the DS tuning method outperforms the AIC method in the regulatory experiment. Notice that even though using the GMS model produces very consistent tunings, with better overall indexes in many experiments, it is outperformed by the Karnopp and Kano models when the PID with CF and CR controller structure is used. 


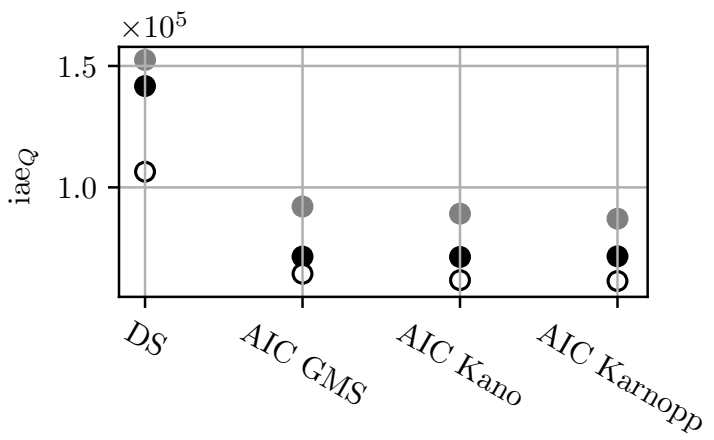

(a) PID.

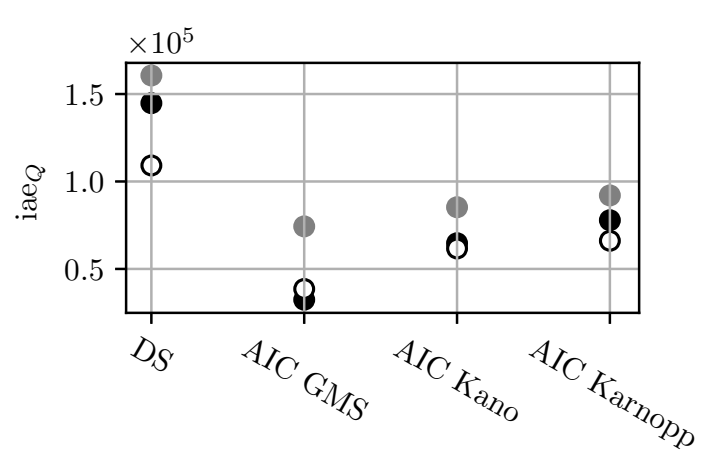

(b) PID with CF.

Figure 6.21 - AIC and DS tuning performance comparison of the PID and PID with $\mathrm{CF}$ control structures, in the robustness test.

Legend: regulatory (white), servo (black) and aleatory (gray) experiments.

Source: from Author.

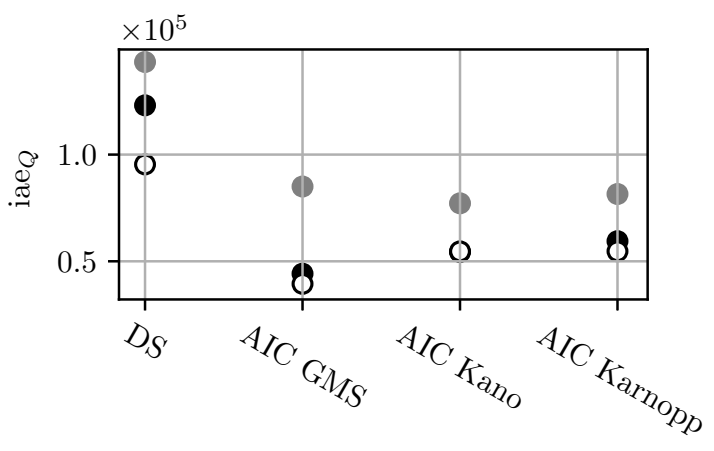

(a) PID with CF and Knocker.

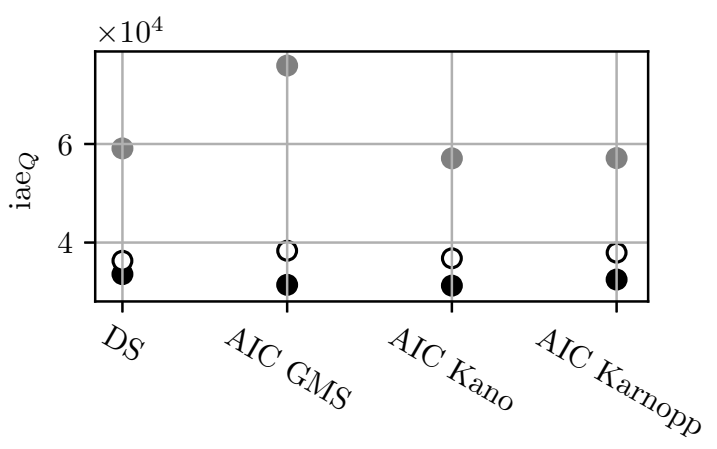

(b) PID with CF and CR.

Figure 6.22 - AIC and DS tuning performance comparison of the PID with CF and Knocker, and the PID with CF and CR, in the robustness test.

Legend: regulatory (white), servo (black) and aleatory (gray) experiments.

Source: from Author.

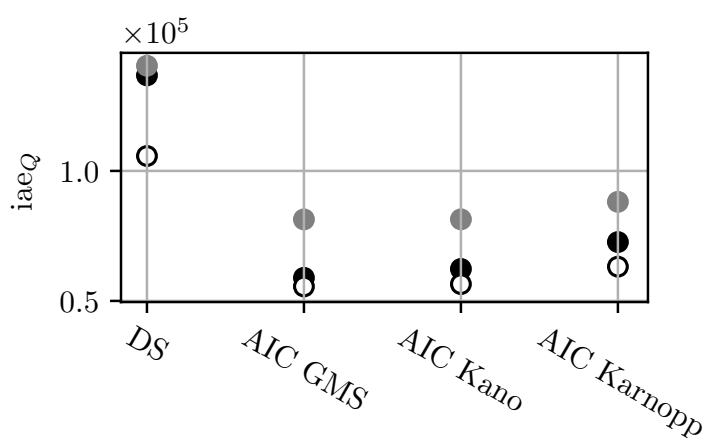

Figure 6.23 - AIC and DS tuning performance comparison of the fuzzy PID control structure, in the robustness test.

Legend: regulatory (white), servo (black) and aleatory (gray) experiments.

Source: from Author. 
An extract of the aleatory experiment, using the PID with CF and CR controller structure, is presented in Figures 6.24 and 6.25. Note that this is the controller structure in which when the Kano and Karnopp friction models are used in the AIC framework, the performance is better than when the GMS friction model is used.

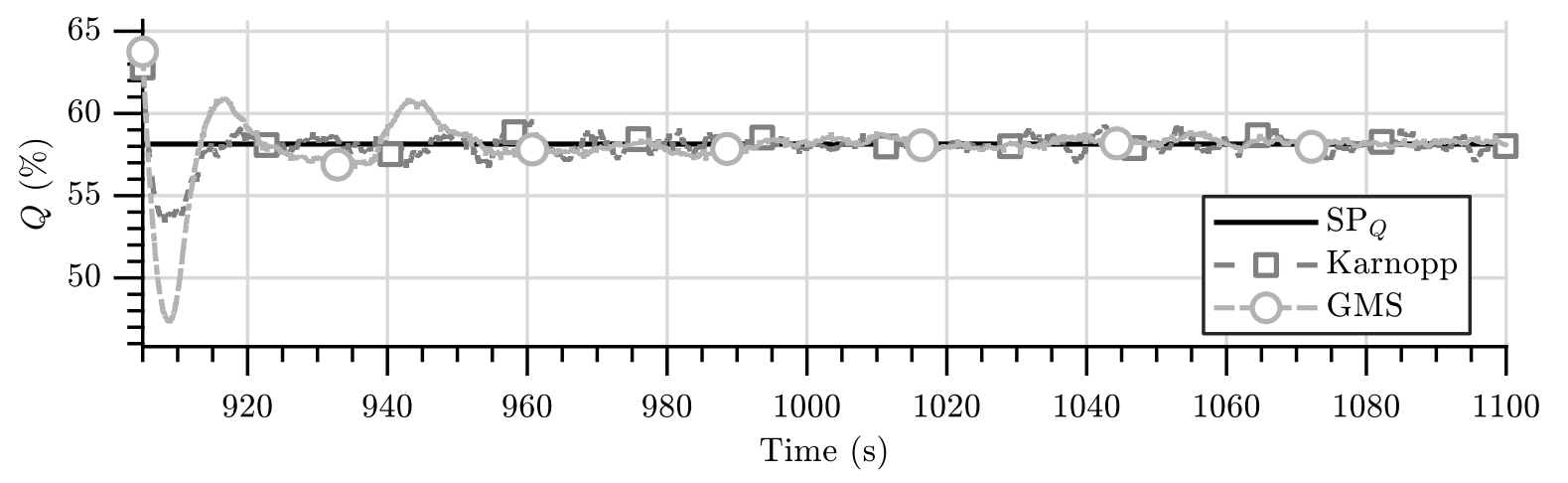

Figure 6.24 - Extract of the aleatory experiment using the PID with CF and CR friction compensation, tuned with the Karnopp and GMS models.

Source: from Author.

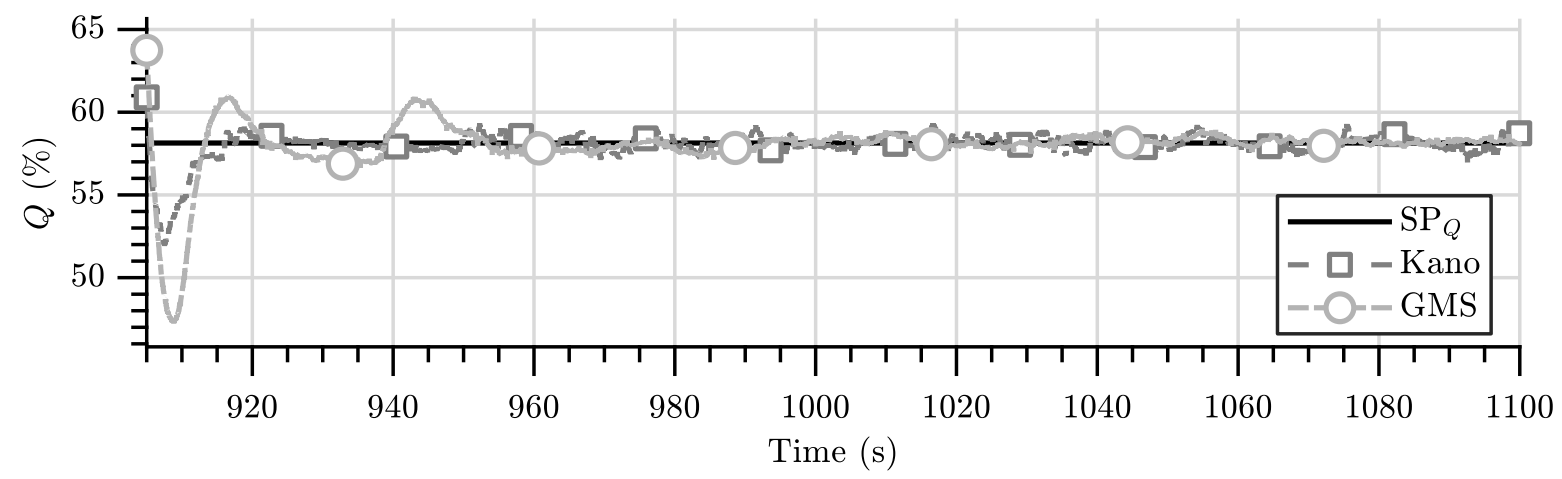

Figure 6.25 - Extract of the aleatory experiment using the PID with CF and CR friction compensation, tuned with the Kano and GMS models.

Source: from Author.

\subsection{Test of the Sliding Mode Control and of the Feedforward PID with Integral Deadband Structure}

In this test, the best control structure so far, the PID with CF and CR, is compared to two other control structures, the Integrative Sliding Mode Control (ISMC) and the Feedforward PID with Integral Deadband controller, which are defined in the following subsections.

\subsubsection{The Integrative Sliding Mode Control (ISMC)}

The ISMC control design starts by the definition of a sliding surface, that in the case of the diaphragm control valve, considering that the I/P converter can be modeled by 
a gain, is:

$$
s_{i s m c}(t)=\dot{\tilde{x}}(t)+2 \lambda_{i s m c} \tilde{x}(t)+\lambda_{i s m c}^{2} \int_{t_{0}}^{t} \tilde{x}(t) \mathrm{d} t,
$$

where $\tilde{x}(t)=\hat{x}(t)-\mathrm{ES}(t), \mathrm{ES}(t)$ is the excitation signal of the AIC framework (or SP for the real valve control system) and $\lambda_{i s m c}$ is a control parameter that defines how fast the stem position tracking error, $\tilde{x}$, decays to zero, given that the system reaches the sliding surface. Taking the time derivative of Eq. (6.14), using the valve model from Eq. (3.1) and neglecting the spring initial force, one obtains:

$$
\dot{s}_{i s m c}(t)=\left(\frac{S_{a} K_{P_{g}}}{m} \hat{\mathrm{OP}}(t)-\frac{k}{m} \hat{x}(t)-\frac{F_{f}(t)}{m}-\ddot{\mathrm{ES}}(t)\right)+2 \lambda_{i s m c} \dot{\tilde{x}}(t)+\lambda_{i s m c}{ }^{2} \tilde{x}(t) .
$$

Then, a control law can be defined to ensure that the sliding surface is attractive. The most straightforward structure that can be used is the feedback linearization:

$$
\begin{aligned}
\hat{\mathrm{OP}}(t)=\frac{\hat{k} \hat{x}(t)+\hat{F}_{f}(t)}{\hat{S}_{a} \hat{K}_{P_{g}}}+\left[\ddot{\mathrm{ES}}(t)-2 \lambda_{i s m c} \dot{\tilde{x}}(t)+\lambda_{i s m c}{ }^{2} \tilde{x}(t)\right] \frac{\hat{m}}{\hat{S}_{a} \hat{K}_{P_{g}}}- \\
k_{i s m c}(\hat{x}, \dot{\hat{x}}) \operatorname{sat}\left(\frac{s_{i s m c}}{\Phi_{\text {ismc }}}\right),
\end{aligned}
$$

where $k_{i s m c}(\hat{x}, \dot{\hat{x}})$ is a gain that may vary with the stem position and velocity, $\Phi_{i s m c}$ is used to build a dead zone around the sliding surface, to avoid chattering, and the saturation function is defined as:

$$
\operatorname{sat}\left(\frac{s_{i s m c}}{\Phi_{i s m c}}\right)= \begin{cases}1, & \text { if } s_{i s m c} / \Phi_{i s m c}>1 \\ -1, & \text { if } s_{i s m c} / \Phi_{i s m c}<-1 \\ s_{i s m c} / \Phi_{i s m c}, & \text { otherwise. }\end{cases}
$$

To ensure that the created sliding surface is an attractor, the following equation has to be true:

$$
\frac{1}{2} \frac{\mathrm{d} s_{i s m c}^{2}}{\mathrm{~d} t}<-\nu_{i s m c}\left|s_{i s m c}\right|
$$

where $\nu_{i s m c}$ defines how fast the system reaches the sliding surface. Using Eq. (6.18) criterion, the sliding surface time derivative, Eq. (6.15), the control law, Eq. (6.16), and selecting a proper value for $\nu_{i s m c}$, one can derive analytically the value of $k_{i s m c}(\hat{x}, \dot{\hat{x}})$, to ensure that the sliding surface is an attractor. Yet, if the mismatch between the plant and the model can be estimated $\left(\max \left(\left|F_{f}-\hat{F}_{f}\right|\right)\right.$, for example), it can be used to ensure that the controller is robust with respect to the model inaccuracies. However, the AIC framework is used to tune the controller, instead of calculating the ISMC parameters using mathematical analysis, as the valve model, in the AIC framework, is considered a very good approximation of the real valve behavior. Yet, in this work, the ISMC gain, $k_{i s m c}$, is considered constant. 
The valve model parameters used in the ISMC control law are the ones estimated in Chapters 4 and 5. The friction model, however, is a simplified Stribeck curve, which is frequently employed to compensate diaphragm control valve friction, defined as (KAYIHAN; DOYLE III, 2000; HIDALGO; GARCIA, 2017; BAEZA; GARCIA, 2018):

$$
\hat{F}_{f}(\dot{\hat{x}})=\left[\hat{F}_{c}+\left(\hat{F}_{s}-\hat{F}_{c}\right) \exp \left\{-\left(\dot{\hat{x}} / \hat{v}_{s}\right)^{2}\right\}\right] \tanh (10000 \dot{\hat{x}})+\hat{F}_{v} \dot{\hat{x}} .
$$

The Stribeck curve parameters are given by the estimated friction model used in the AIC framework, when the Karnopp and GMS models are used. Instead, if the Kano model is used, then $\hat{F}_{v}=0 \mathrm{Ns} / \mathrm{m}$ and $\hat{v}_{s}=10^{-6} \mathrm{~m} / \mathrm{s}$ and the Coulomb and static friction parameters are calculated using the Kano parameters, as presented in Section 3.3.

Besides the ISMC parameters, $k_{i s m c}, \lambda_{i s m c}$ and $\Phi_{i s m c}$, the control law relies on estimating the stem velocity, as shown in Eq. (6.16), which is calculated using a backward differentiation of the filtered stem position using a low pass first order filter, whose passband $\left(1 / \tau_{\text {ismc }} \mathrm{rad} / \mathrm{s}\right)$ is also tuned using the AIC framework. It is worth mentioning that the valve set point has to be twice differentiable, therefore, it is filtered using a second order low pass filter, with unitary relative damping and natural frequency equal to $2 / \hat{\tau}_{\mathrm{I} / \mathrm{P}} \mathrm{rad} / \mathrm{s}$. The search space of the ISMC controller structure is presented in Table 6.11.

Table 6.11 - ISMC parameter search space.

\begin{tabular}{ccc} 
Parameter & $u b_{d}$ & $l b_{d}$ \\
\hline$k_{\text {ismc }}$ & 2000 & 1 \\
$\lambda_{\text {ismc }}$ & 2000 & $10^{-3}$ \\
$\Phi_{\text {ismc }}$ & 1 & $10^{-4}$ \\
$\tau_{\text {ismc }}$ & $\hat{\tau}_{\mathrm{I} / \mathrm{P}}$ & $\hat{\tau}_{\mathrm{I} / \mathrm{P}} / 100$ \\
\hline
\end{tabular}

\subsubsection{The Feedforward PID with Integral Deadband (FFPID) Controller}

This control structure uses the same PID defined in Section 6.2, however, its integral action has a deadband, where only absolute errors higher than a certain threshold $\left(e_{I_{t h}}\right)$ are integrated. The integral deadband is widely used in the industry to reduce the control loop variability. Therefore, the integral term of the PID, defined in Eq. (6.5), is redefined to:

$$
I_{C_{p}}[n]= \begin{cases}K_{i} \frac{T_{s}}{2}(\hat{e}[n]+\hat{e}[n-1])+I_{C_{p}}[n-1] & , \text { if }|\hat{e}[n]|>e_{I_{t h}} \\ I_{C_{p}}[n-1] & , \text { otherwise }\end{cases}
$$

The feedforward term is a function of ES, that is added directly to the PID output. As the valve model is known, it is possible to calculate the diaphragm pressure that is necessary to reach the desired $\operatorname{ES}[n]$ :

$$
\hat{P}_{f f}[n]=\frac{1}{\hat{S}_{a}}\left[\hat{k} \mathrm{ES}[n]+\hat{F}_{f}(n, \dot{\mathrm{ES}})+\hat{F}_{\mathrm{init}}+\hat{m} \ddot{\mathrm{ES}}[n]\right] .
$$


As the friction models have some internal states that are hard to be estimated in practice, once more the friction force is modeled using the Stribeck curve:

$$
\hat{F}_{f}(\operatorname{ES}[n])=\left[\hat{F}_{c}+\left(\hat{F}_{s}-\hat{F}_{c}\right) \exp \left\{-\left(\operatorname{ES}[n] / \hat{v}_{s}\right)^{2}\right\}\right] \operatorname{sgn}(\operatorname{ES}[n])+\hat{F}_{v} \operatorname{ES}[n] .
$$

As in the practical application the set point is given by $C_{H}$, it may propagate noise from the hydraulic controlled variable measurement system, then ES is filtered using a first order low pass filter with $5 / \hat{\tau}_{\mathrm{I} / \mathrm{P}} \mathrm{rad} / \mathrm{s}$ passband. The normalized feed forward value, $\hat{\mathrm{OP}}_{f f}[n]$, is easily derived from $\hat{P}_{f f}[n]$, considering that the I/P converter has instantaneous response:

$$
\hat{\mathrm{OP}}_{f f}[n]=\left(\frac{\hat{P}_{f f}[n]}{\hat{S}_{a}}-\hat{P}_{\mathrm{min}}\right) \frac{1}{\hat{K}_{P_{g}}} k_{f f},
$$

where $k_{f f}$ is the feedforward gain, that is used to compensate some inaccuracies that the feedforward model has, such as the assumptions that the friction force can be modeled by the Stribeck curve and the I/P model instantaneous response.

The search space of the FFPID parameters is presented in Table 6.12, and as this structure is based on the PID controller, the search space shown in Table 6.1 is also applicable to the FFPID.

Table 6.12 - FFPID parameter search space.

\begin{tabular}{ccc} 
Parameter & $u b_{d}$ & $l b_{d}$ \\
\hline$e_{I_{t h}}$ & 0.5 & 0 \\
$k_{f f}$ & 1 & 0.5 \\
\hline
\end{tabular}

\subsubsection{Test Results}

For each controller, the parameters are tunned using the AIC framework, using the open and closed loop estimated valve model. Unlike the PID tests, only $\hat{\tau}_{\mathrm{I} / \mathrm{P}_{r}}$ is used in the open loop valve model, as it has the best performance so far. The AIC tunings, for each control structure and valve model, are presented in Tables 6.13 to 6.15. Regarding the PID with CF and CR tunings, notice that when the closed loop estimated valve model in the AIC framework is used, the $a_{C R}$ is closer to the analytical value that is suggested in the literature, whereas when the open loop estimated valve model is used instead, the CR action is almost inexistent. It is interesting to note the large controller tuning variance among the estimated models used in the AIC framework, for the ISMC and FFPID control structures. 
Table 6.13 - PID with CF and CR friction compensation tunings in the ISMC and FFPID tests.

\begin{tabular}{lcrccrcrrr} 
Model & $K_{p}{ }^{*}$ & \multicolumn{1}{c}{$K_{i}{ }^{*}$} & $K_{d}{ }^{*}$ & $\tau_{d f}{ }^{*}$ & $e_{l}{ }^{*}$ & $e_{h}{ }^{*}$ & $t_{e_{l}}{ }^{*}$ & $t_{e_{h}}{ }^{*}$ & $a_{C R}{ }^{*}$ \\
\hline Kano OL & 5.78 & 11.89 & 4.23 & 2.51 & 3.00 & 1.76 & 0.13 & 0.04 & 2.13 \\
Kano CL & 2.63 & 10.94 & 2.33 & 2.17 & 2.31 & 1.58 & 9.16 & 0.75 & 11.40 \\
Karnopp OL & 8.34 & 23.48 & 4.23 & 1.16 & 6.43 & 0.11 & 0.10 & 0.08 & 0.01 \\
Karnopp CL & 4.24 & 7.32 & 0.36 & 0.09 & 9.99 & 1.05 & 0.14 & 0.01 & 11.60 \\
GMS OL & 7.48 & 19.57 & 4.02 & 1.65 & -0.84 & 0.06 & 3.59 & 0.85 & 0.50 \\
GMS CL & 4.33 & 9.06 & 0.36 & 0.08 & 7.62 & 0.44 & 16.59 & 0.93 & 7.15 \\
\hline \multicolumn{7}{c}{ Source: from Author. }
\end{tabular}

OL: model with open loop estimated parameters and CL: model with closed loop estimated parameters.

Table 6.14 - ISMC tunings in the ISMC and FFPID tests.

\begin{tabular}{lrrrr} 
Model & $k_{i s m c}{ }^{*}$ & $\lambda_{i s m c}{ }^{*}$ & $\Phi_{i s m c}{ }^{*}$ & $\tau_{\text {ismc }}{ }^{*}$ \\
\hline Kano OL & 5.28 & 1911.97 & 0.58 & 0.42 \\
Kano CL & 1.75 & 1353.64 & 0.07 & 0.22 \\
Karnopp OL & 1991.28 & 0.19 & 0.61 & 0.004 \\
Karnopp CL & 4.27 & 1994.28 & 0.81 & 0.23 \\
GMS OL & 1783.40 & 0.87 & 0.53 & 0.004 \\
GMS CL & 3.28 & 1950.57 & 0.99 & 0.22 \\
\hline \multicolumn{5}{c}{ Source: from Author. }
\end{tabular}

OL: model with open loop estimated parameters and CL: model with closed loop estimated parameters.

Table 6.15 - FFPID tunings in the ISMC and FFPID tests.

\begin{tabular}{lcccccc} 
Model & $K_{p}{ }^{*}$ & $K_{i}{ }^{*}$ & $K_{d}{ }^{*}$ & $\tau_{d f}{ }^{*}$ & $e_{I_{t h}}{ }^{*}$ & $k_{f f}{ }^{*}$ \\
\hline Kano OL & 5.48 & 0.77 & 0.80 & 0.14 & 0.32 & 0.60 \\
Kano CL & 2.47 & 0.10 & 0.17 & 0.03 & 0.01 & 0.76 \\
Karnopp OL & 5.06 & 0.40 & 0.80 & 0.15 & 0.22 & 0.94 \\
Karnopp CL & 2.40 & 0.00 & 0.15 & 0.16 & 0.18 & 0.99 \\
GMS OL & 4.80 & 0.31 & 0.64 & 0.08 & 0.45 & 0.89 \\
GMS CL & 5.29 & 2.30 & 0.33 & 0.04 & 0.39 & 0.57 \\
\hline \multicolumn{6}{c}{ Source: from Author. }
\end{tabular}

OL: model with open loop estimated parameters and CL: model with closed loop estimated parameters.

The same tests described in Section 6.2 are performed. A performance comparison between the open and closed loop estimated model parameters used in the AIC framework is presented in Figure 6.26. Using the open loop estimated parameters in the AIC framework, in general, results in slightly better performance indexes. However, this improved performance has a drawback, which is a higher control effort. 


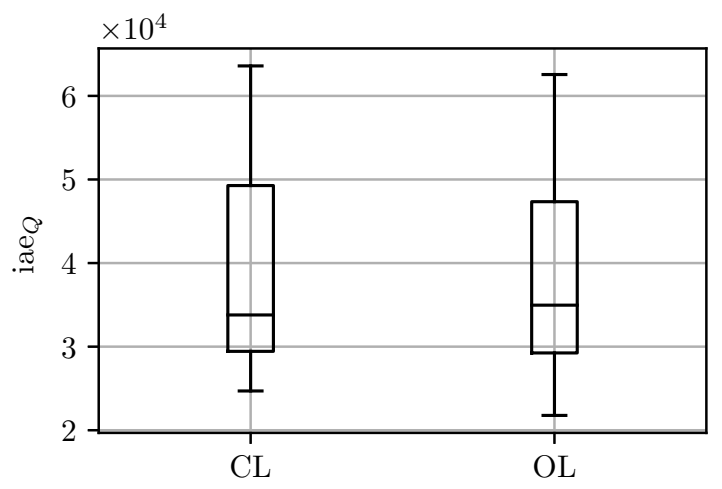

(a) Comparison of the $\operatorname{iae}_{Q}$.

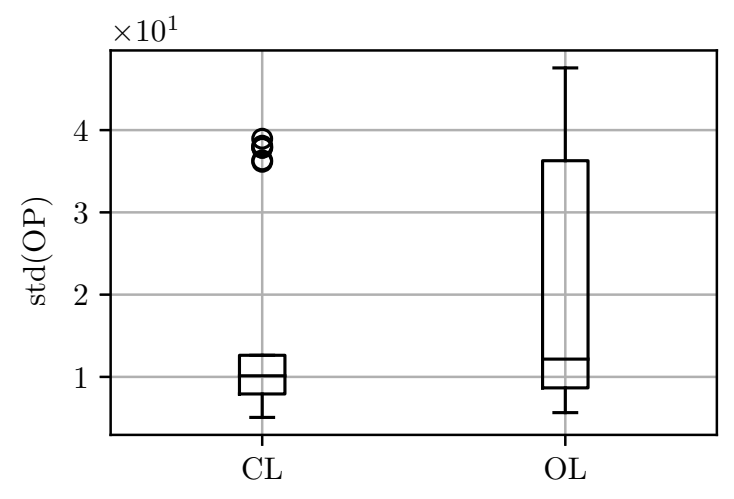

(b) Comparison of the $\operatorname{std}(\mathrm{OP})$.

Figure 6.26 - Comparison of tuning performance when the open and closed loop estimated valve models are used in the AIC framework.

Source: from Author.

A comparison among the AIC tuning performance, for when different estimated valve models are used, is presented in Figures 6.27 and 6.28. The GMS holds the best overall performance among the models, regarding the $i_{Q} e_{Q}$ index, whether the open or closed loop estimated valve models are used, however, in general, using the GMS and Karnopp models in the AIC framework result in similar performance indexes, as the first and third quartiles are close to each other. When the Kano model is used in the AIC framework, the hydraulic control performance tends to decrease.

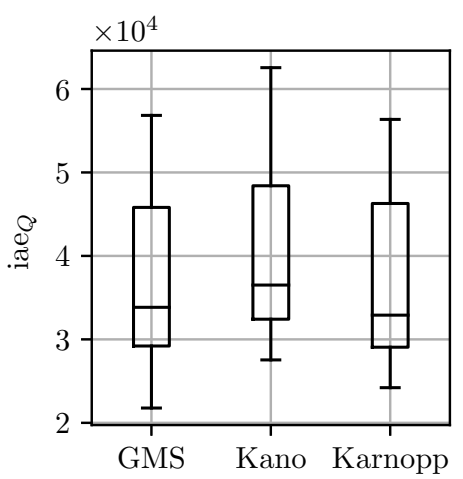

(a) Comparison of the $\operatorname{iae}_{Q}$.

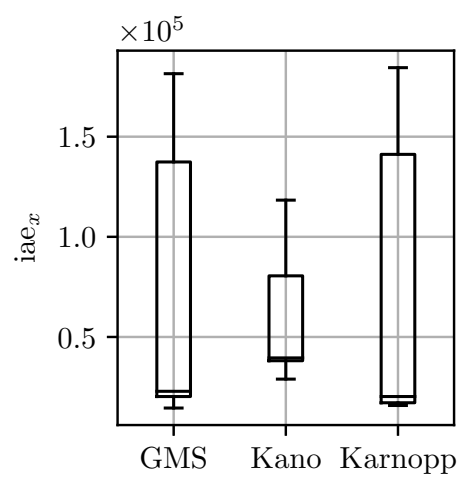

(b) Comparison of the iae $_{x}$.

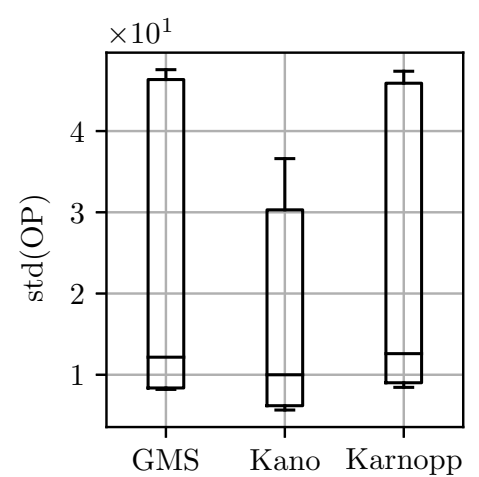

(c) Comparison of the $\operatorname{std}(\mathrm{OP})$.

Figure 6.27 - Comparison of the controller tuning performance, for each open loop estimated friction model used in the AIC framework.

Source: from Author. 


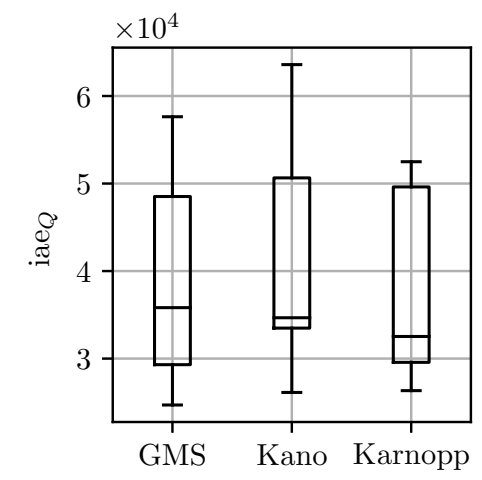

(a) Comparison of the $\operatorname{iae}_{Q}$.

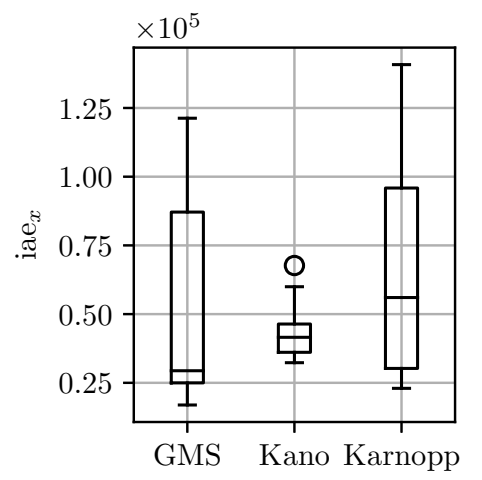

(b) Comparison of the iae ${ }_{x}$.

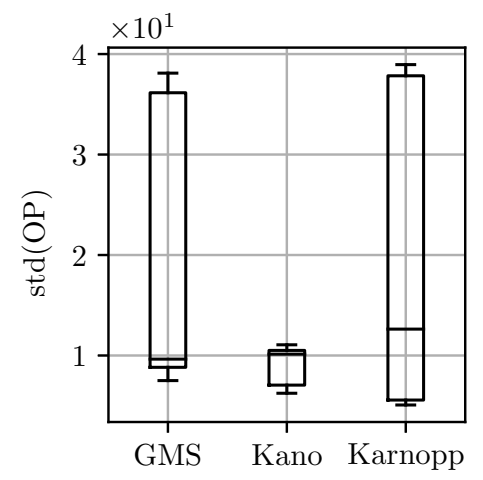

(c) Comparison of the std(OP).

Figure 6.28 - Comparison of the controller tuning performance, for each closed loop estimated friction model used in the AIC framework.

Source: from Author.

The AIC tuning performance, for each experiment type, when the open loop estimated valve models are used, is presented in Figure 6.29. The data shows that it is more likely to obtain better values of the iae $_{Q}$ index when the GMS friction model is used in the AIC framework, specially regarding the servo and regulatory experiments.

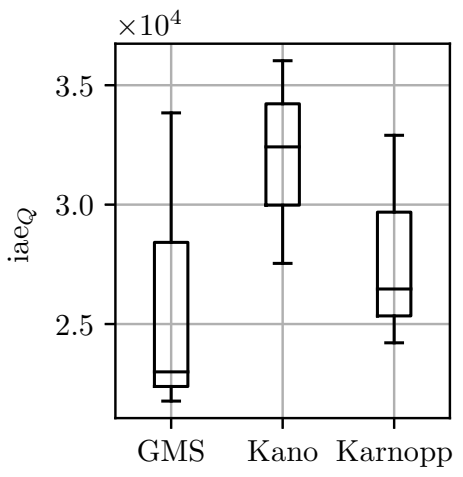

(a) Comparison of the $\operatorname{iae}_{Q}$, in the servo experiment.

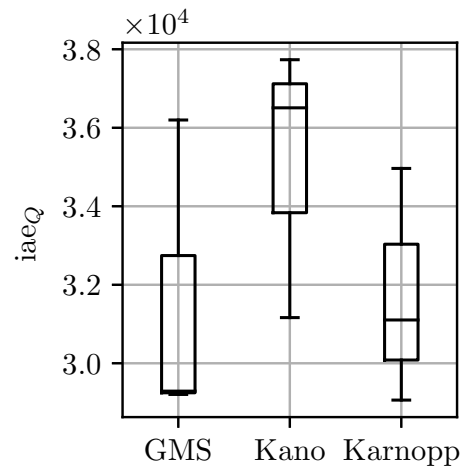

(b) Comparison of the iae $_{Q}$, in the regulatory experiment.

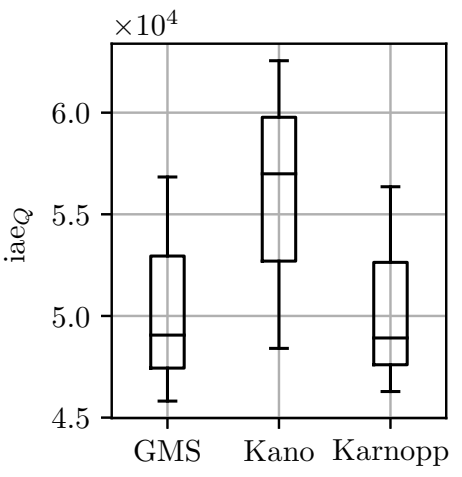

(c) Comparison of the iae $_{Q}$, in the aleatory experiment.

Figure 6.29 - Comparison of the controller tuning performance, separated by experiment type, for each open loop estimated friction model used in the AIC.

Source: from Author.

Figures 6.30 to 6.35 present performance comparisons of the controller structures, for each estimated friction model used in the AIC framework. The ISMC structure has the higher control effort, whichever the friction model is used to tune its parameters and this increased control effort does not necessarily corresponds to a better hydraulic system control performance. When the GMS model is used, the best performance indexes are obtained using the PID with CF and CR control structure, even though the FFPID outperforms in the servo experiment when using the open loop estimated GMS model and 
the ISMC outperforms in the servo experiment when using the closed loop estimated GMS model. When the Karnopp model is used, the best results are obtained with the FFPID and ISMC control structures, when the open or closed loop estimated valve model is used in the AIC framework, respectively. In general, the GMS and Karnopp models present similar patterns regarding the FFPID and ISMC controller structures, as when the open loop estimated friction models are used, the FFPID outperforms the ISMC, whereas when the closed loop estimated friction models are used, the ISMC outperforms the FFPID. The Kano model, however, presents a different pattern, since when the open loop estimated friction model is used, the best performance is given by the ISMC control structure, while when the closed loop estimated friction model is used, the best performance is given by the PID with CF and CR. What is impressive regarding the PID with CF and CR control structure is its high consistency, specially when the GMS and Karnopp estimated models are used in the AIC framework. It presents a very similar performance index than the more complex control structures, however its control effort is much lower than the other structures.

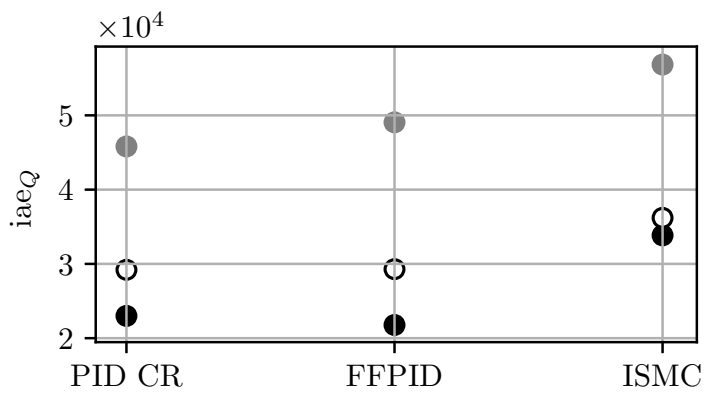

(a) Comparison of the $\operatorname{iae}_{Q}$.

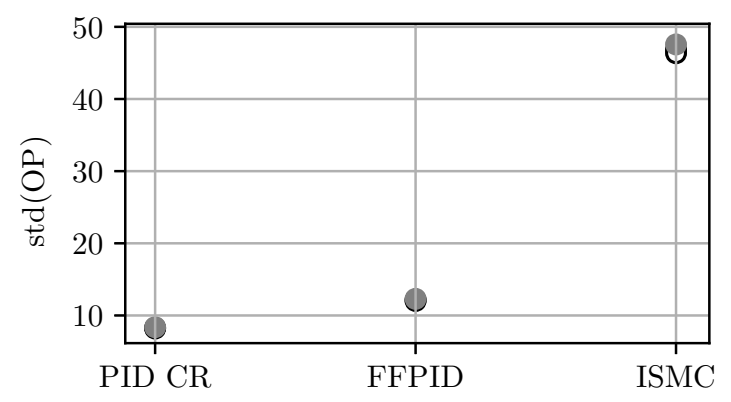

(b) Comparison of the std(OP).

Figure 6.30 - Comparison of the tuning performance when the open loop estimated GMS model is used in the AIC framework.

Legend: regulatory (white), servo (black) and aleatory (gray) experiments. Source: from Author. 


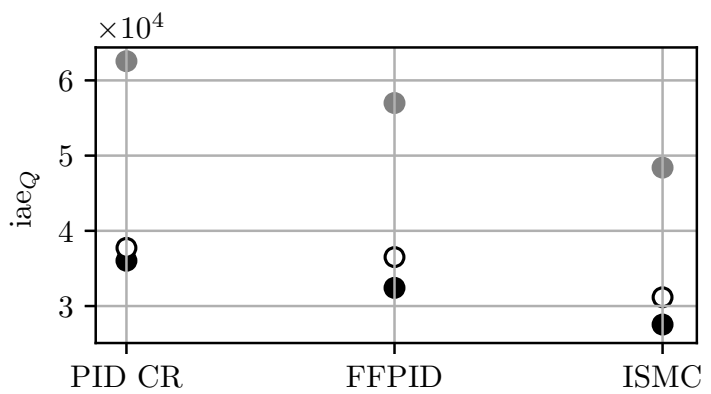

(a) Comparison of the $\operatorname{iae}_{Q}$.

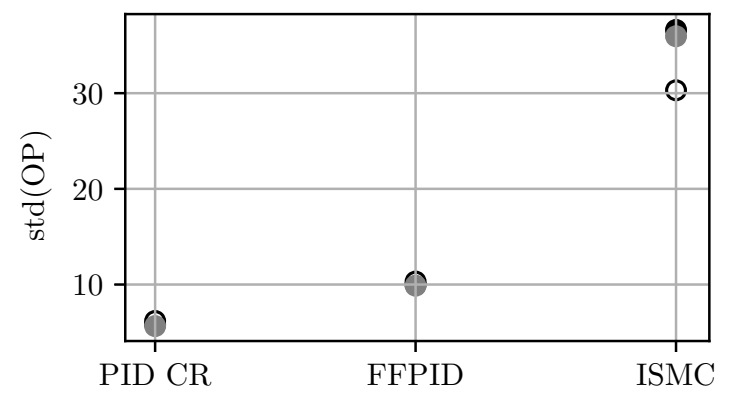

(b) Comparison of the $\operatorname{std}(\mathrm{OP})$.

Figure 6.31 - Comparison of the tuning performance when the open loop estimated Kano model is used in the AIC framework.

Legend: regulatory (white), servo (black) and aleatory (gray) experiments.

Source: from Author.

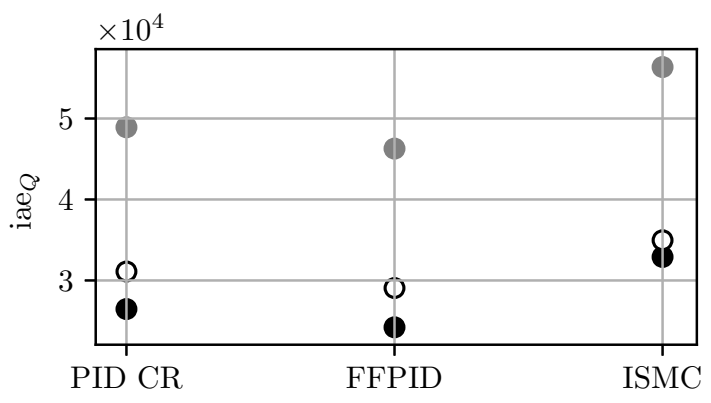

(a) Comparison of the $\operatorname{iae}_{Q}$.

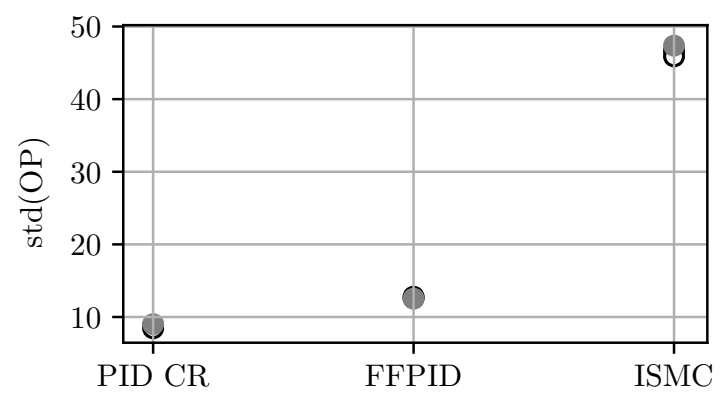

(b) Comparison of the $\operatorname{std}(\mathrm{OP})$.

Figure 6.32 - Comparison of the tuning performance when the open loop estimated Karnopp model is used in the AIC framework.

Legend: regulatory (white), servo (black) and aleatory (gray) experiments.

Source: from Author.

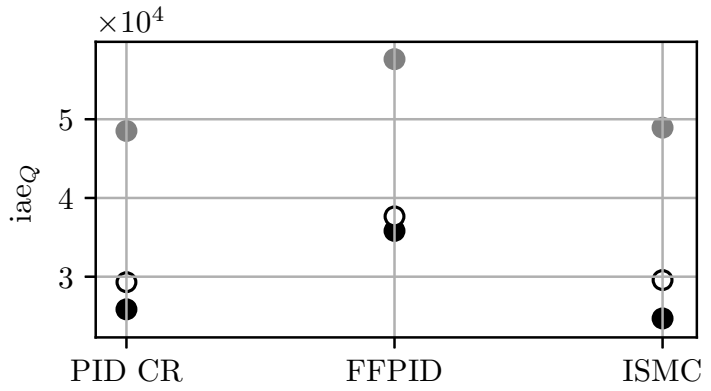

(a) Comparison of the $\operatorname{iae}_{Q}$.

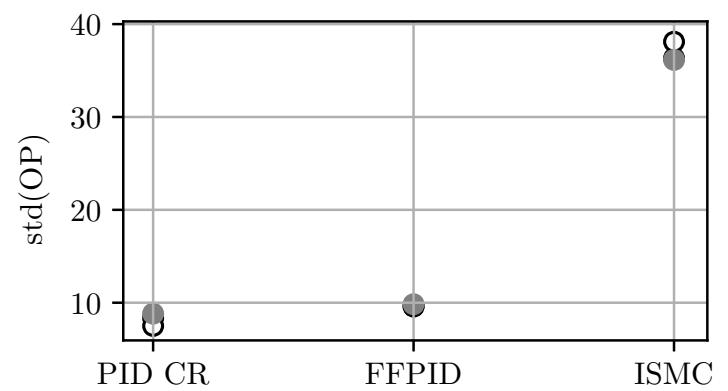

(b) Comparison of the std(OP).

Figure 6.33 - Comparison of the tuning performance when the closed loop estimated GMS model is used in the AIC framework.

Legend: regulatory (white), servo (black) and aleatory (gray) experiments.

Source: from Author. 


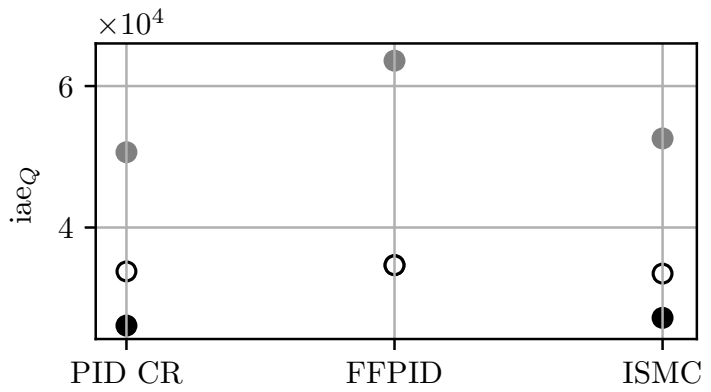

(a) Comparison of the $\operatorname{iae}_{Q}$.

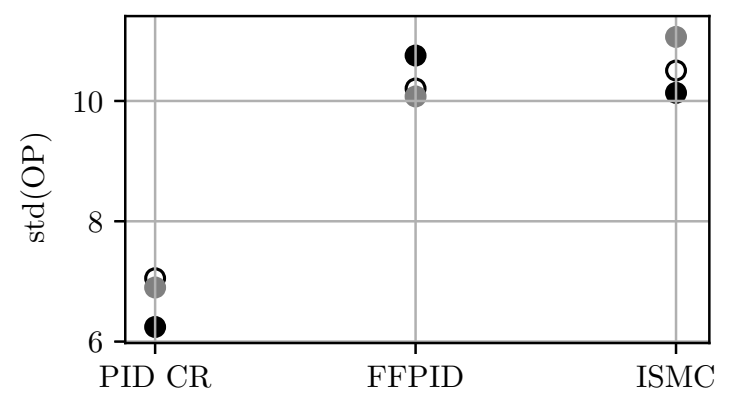

(b) Comparison of the $\operatorname{std}(\mathrm{OP})$.

Figure 6.34 - Comparison of the tuning performance when the closed loop estimated Kano model is used in the AIC framework.

Legend: regulatory (white), servo (black) and aleatory (gray) experiments.

Source: from Author.

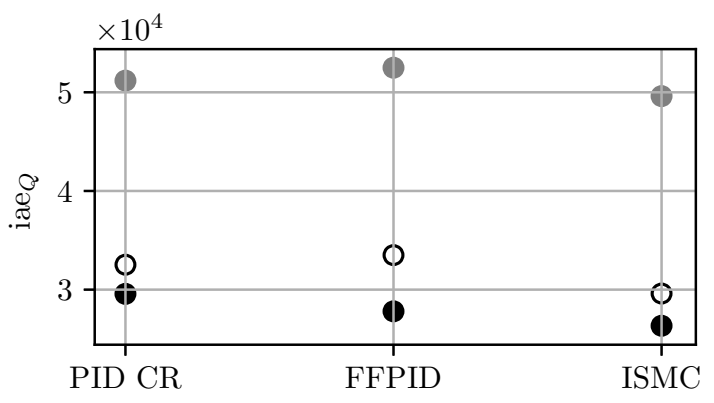

(a) Comparison of the $\operatorname{iae}_{Q}$.

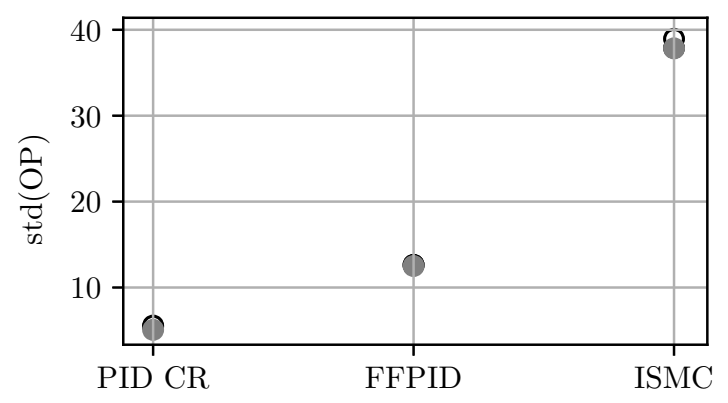

(b) Comparison of the $\operatorname{std}(\mathrm{OP})$.

Figure 6.35 - Comparison of the tuning performance when the closed loop estimated Karnopp model is used in the AIC framework.

Legend: regulatory (white), servo (black) and aleatory (gray) experiments.

Source: from Author.

To study whether there are differences among the estimated valve models used in the AIC framework, a performance comparison, grouping the results by controller structure, is presented in Figures 6.36 and 6.37. Regarding the PID with CF and CR control structure, the best performance in each experiment is given by tuning the controller using the GMS model in the AIC framework, independently whether the model is estimated in open or closed loop. With respect to the FFPID control structure, except for the servo experiment using open loop estimated models in the AIC framework, the best results are obtained when the controller is tuned using the Karnopp model in the AIC framework. As for when the ISMC control structure is used, the best performance occurs when the open loop estimated Kano model or the closed loop estimated GMS model is used. In summary, when the GMS, Kano and Karnopp models are used in the AIC framework, it results in 10, 3 and 5 best performance indexes, when comparing the results by experiment type. 


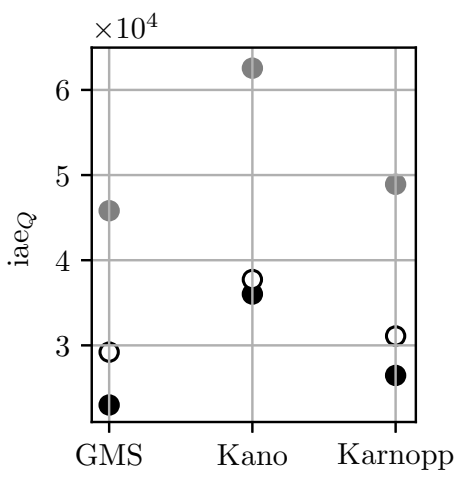

(a) Comparison of the $\operatorname{iae}_{Q}$, for the PID with CF and CR control structure.

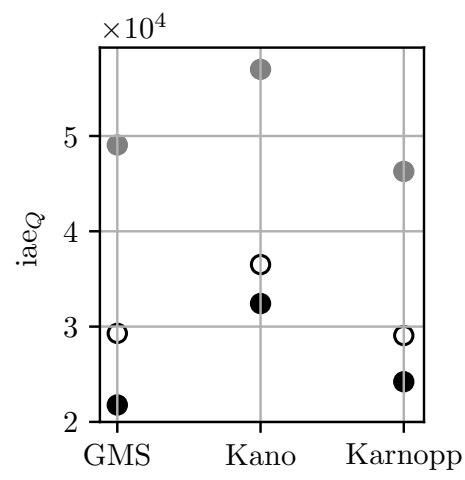

(b) Comparison of the $\operatorname{iae}_{Q}$, for the FFPID control structure.

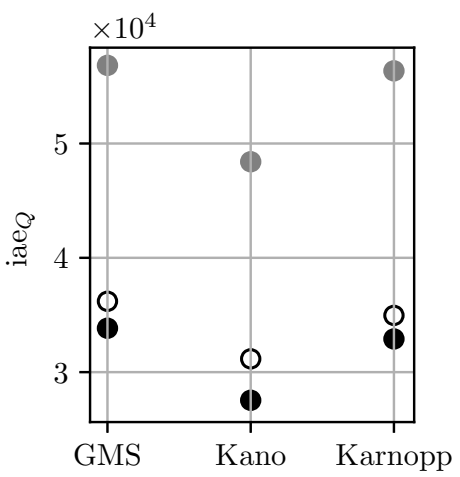

(c) Comparison of the $\operatorname{iae}_{Q}$, for the ISMC control structure.

Figure 6.36 - Comparison of the controller tuning performance when different open loop estimated friction models are used in the AIC framework.

Legend: regulatory (white), servo (black) and aleatory (gray) experiments.

Source: from Author.

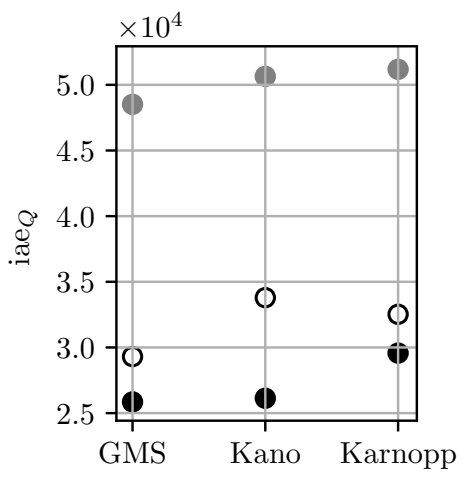

(a) Comparison of the $\operatorname{iae}_{Q}$, for the PID with CF and CR control structure.

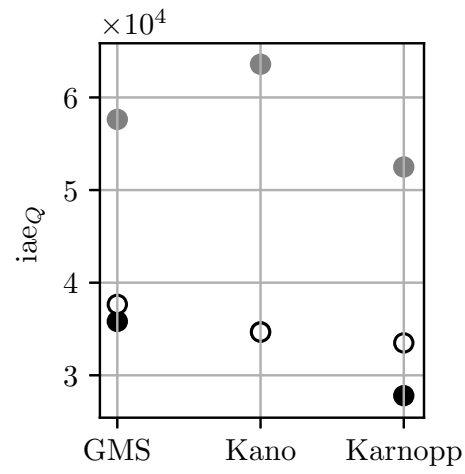

(b) Comparison of the $\operatorname{iae}_{Q}$, for the FFPID control structure.

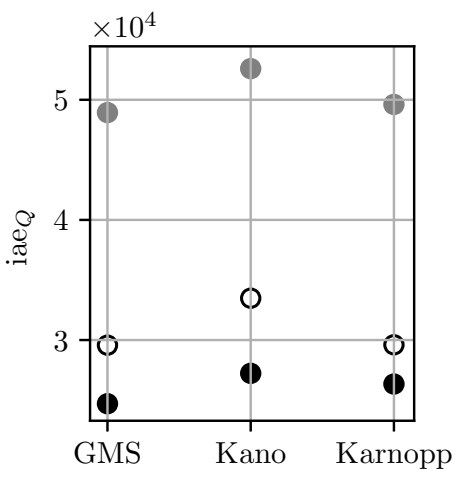

(c) Comparison of the $\operatorname{iae}_{Q}$, for the ISMC control structure.

Figure 6.37 - Comparison of the controller tuning performance when different closed loop estimated friction models are used in the AIC framework.

Legend: regulatory (white), servo (black) and aleatory (gray) experiments.

Source: from Author.

As an example of how the controllers perform in the time domain, an extract of the servo experiment is presented in Figures 6.38 to 6.43. Note that the ISMC has an aggressive tuning, as it tries to compensate even minor deviations from the set point. This characteristic tends to be minimized when using a lower sampling time for the ISMC, which is not possible with the current available FPP hardware. 


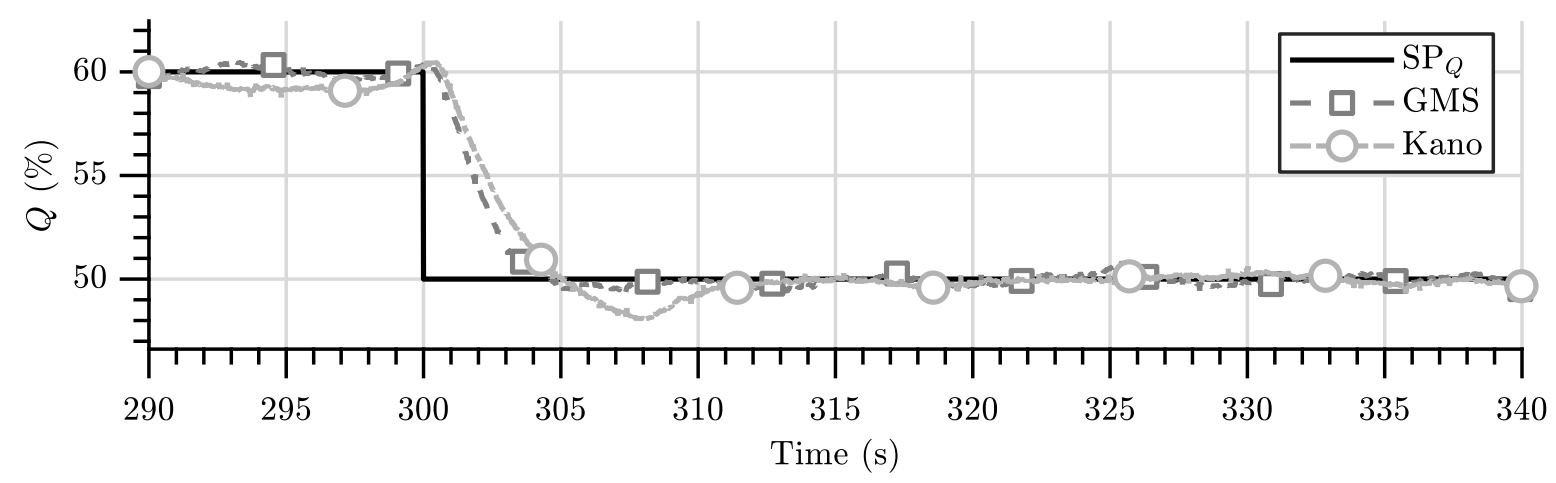

Figure 6.38 - Extract of the servo experiment using the PID with CF and CR, tuned using the open loop estimated GMS and Kano models in the AIC framework.

Source: from Author.

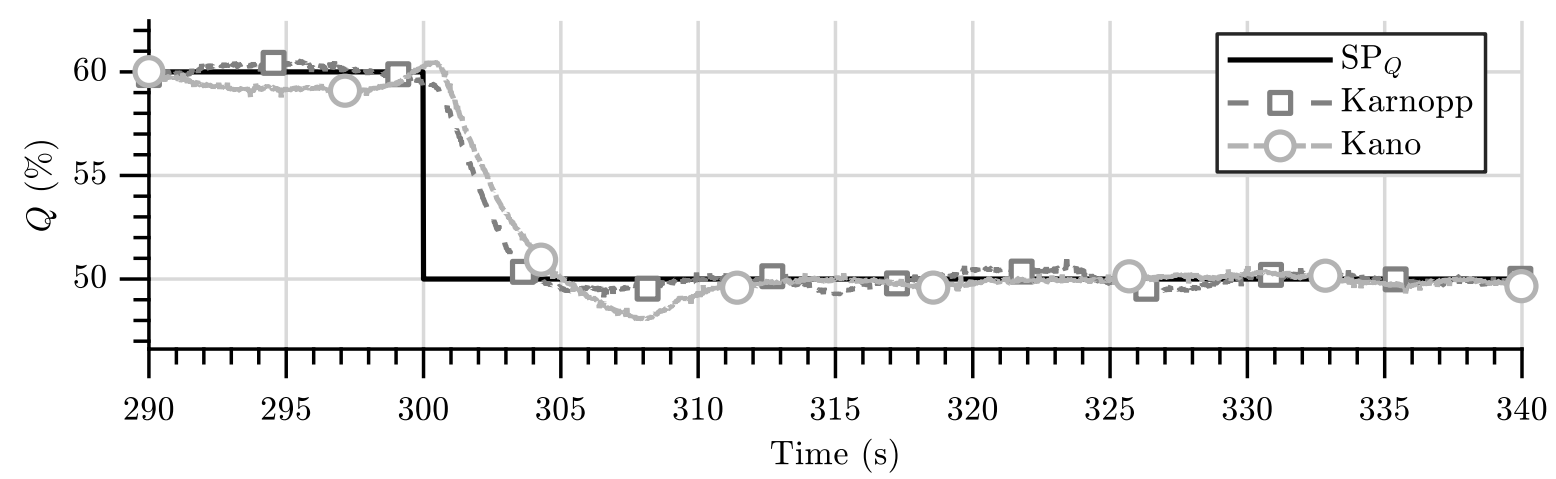

Figure 6.39 - Extract of the servo experiment using the PID with CF and CR, tuned using the open loop estimated Kano and Karnopp models in the AIC framework.

Source: from Author.

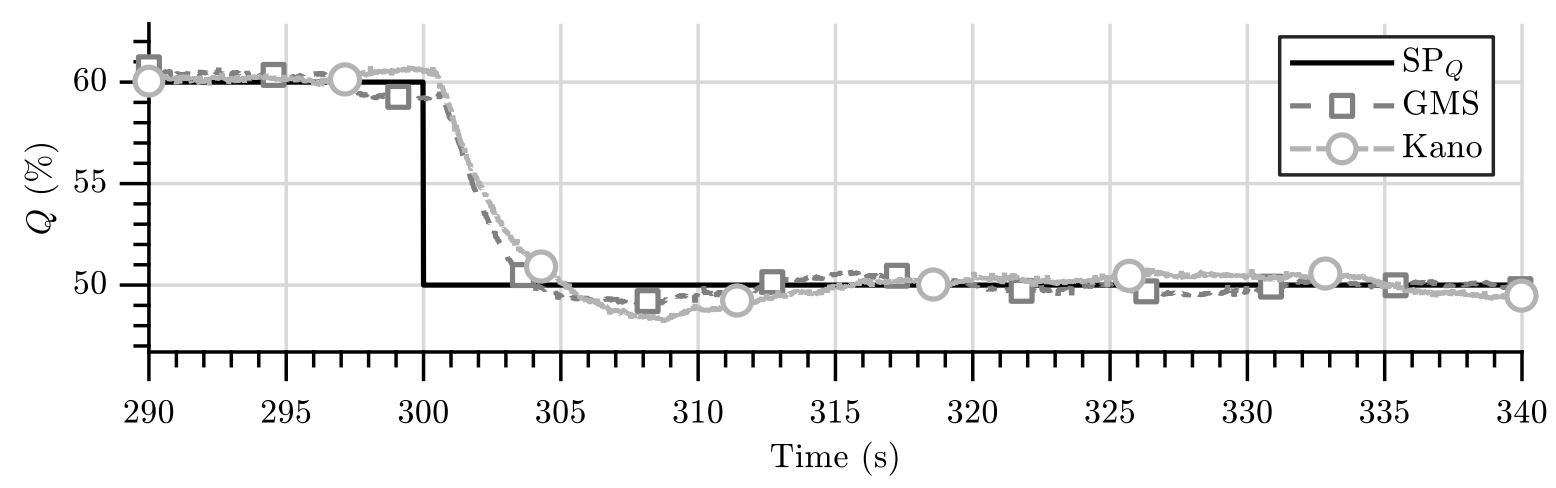

Figure 6.40 - Extract of the servo experiment using the FFPID, tuned using the open loop estimated GMS and Kano models in the AIC framework.

Source: from Author. 


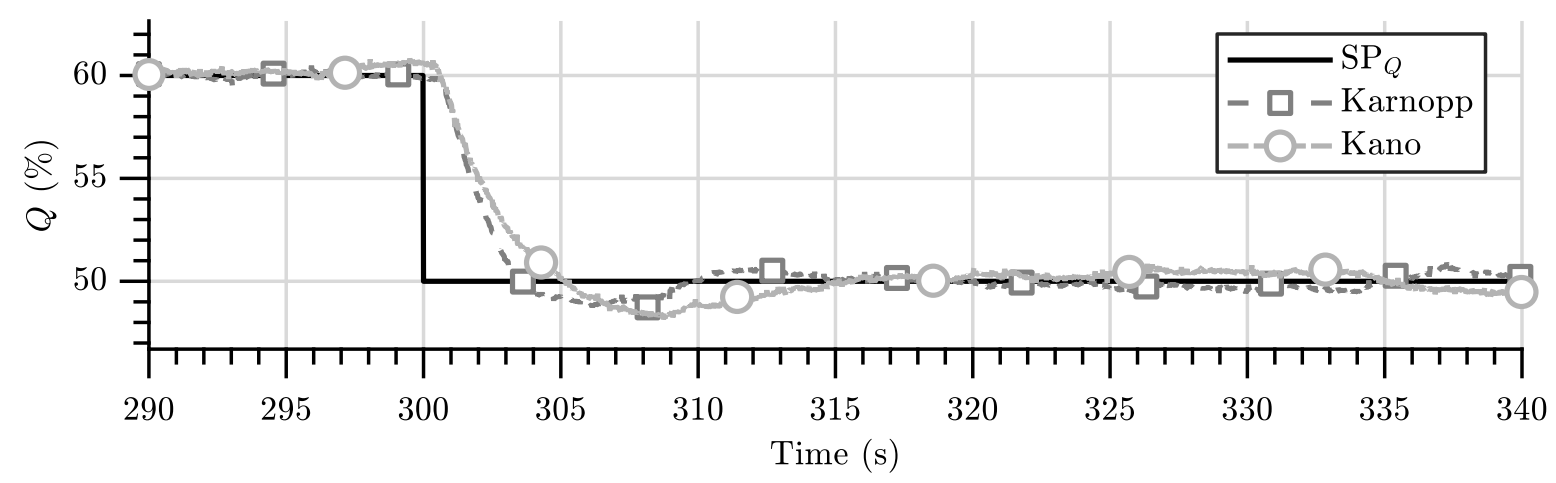

Figure 6.41 - Extract of the servo experiment using the FFPID, tuned using the open loop estimated Kano and Karnopp models in the AIC framework.

Source: from Author.

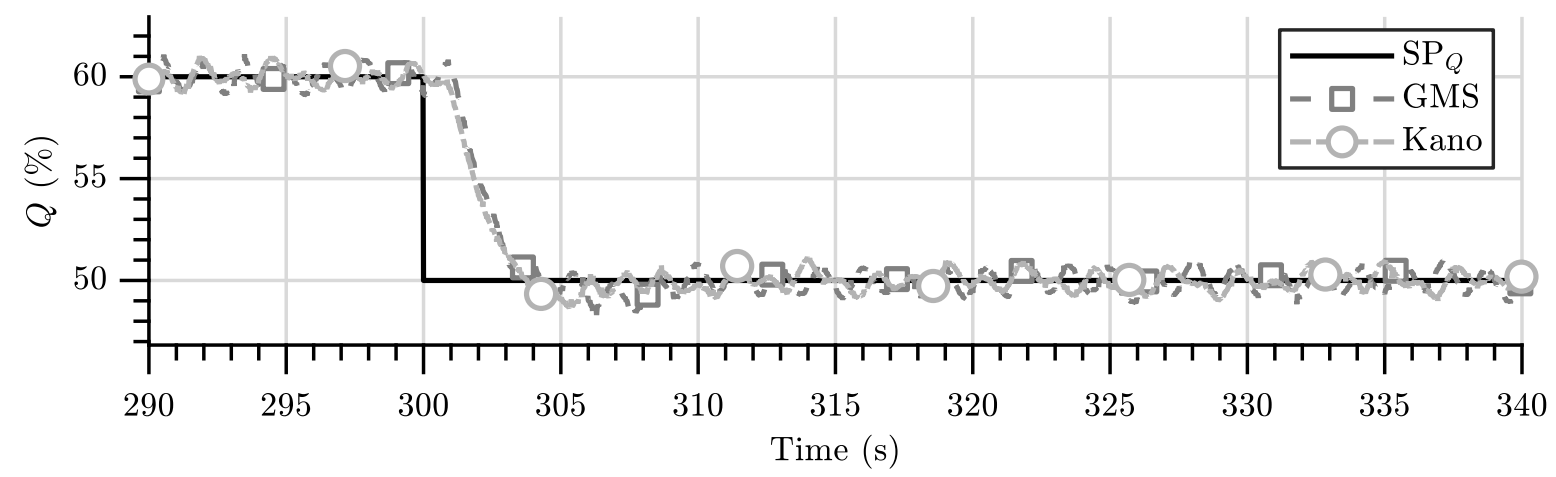

Figure 6.42 - Extract of the servo experiment using the ISMC, tuned using the open loop estimated GMS and Kano models in the AIC framework.

Source: from Author.

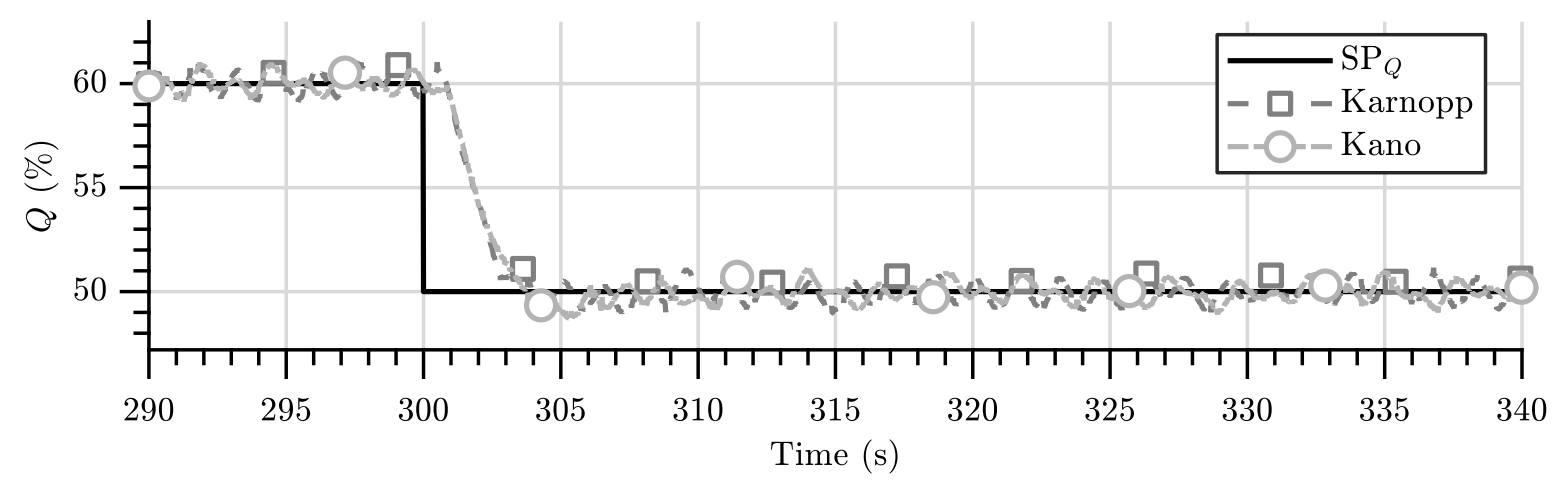

Figure 6.43 - Extract of the servo experiment using the ISMC, tuned using the open loop estimated Kano and Karnopp models in the AIC framework.

Source: from Author. 


\subsection{Test with the Teflon Gasket Valve}

As the teflon gasket valve has a low friction index, it is very simple to control, therefore only a few tests are carried out, to test the AIC framework performance when the valve model is simpler. First a PID and a PID with CF and CR are tuned using the DS method and to do so, a first order plus dead time model of the valve is estimated using a step excitation signal. The first order plus dead time model of the teflon gasket valve is (in the Laplace domain):

$$
\frac{\mathrm{OP}_{t}(s)}{x_{t}(s)}=\frac{0.68914}{0.8305 s+1} \mathrm{e}^{-0.16 s}
$$

Applying the DS method to obtain a closed loop time constant $50 \%$ faster than the model time constant, a PI controller is calculated as $K_{p}=2.10$ and $K_{i}=2.55$. The PID with $\mathrm{CF}$ and CR has the same PID parameters, the CF algorithm is tuned with $e_{l}=e_{h}=1$, $t_{e_{l}}=10 \mathrm{~s}$ and $t_{e_{h}}=2 \mathrm{~s}$, and the CR with $a_{C R}=0.26$.

The control structures tested in the AIC framework are the PID with CR and $\mathrm{CF}$, the ISMC and the FFPID. Only the Kano model is used in the AIC framework, for simplicity purposes, and the open loop I/P valve model uses the time constant estimated with ramps input signal $\left(\hat{\tau}_{\mathrm{I} / \mathrm{P}_{r}}\right)$. The resulting tunings are presented in Tables 6.16 to 6.18. When the open loop estimated Kano model is used in the AIC framework, to tune the PID with CF and CR, the CF algorithm is practically disabled (negative value of $e_{h}$ ). The AIC delivers a CR constant that is somewhat close to what is suggested in the literature. The ISMC tunings vary greatly when using different estimated valve models in the AIC framework and the FFPID tunings are pretty consistent among the open and closed loop estimated models used in the AIC framework.

Table 6.16 - PID with CF and CR friction compensation tunings in the teflon gasket valve test.

\begin{tabular}{lrrrrrrrrr} 
Model & $K_{p}$ & $K_{i}$ & $K_{d}$ & $\tau_{d f}$ & $e_{l}$ & $e_{h}$ & $t_{e_{l}}$ & $t_{e_{h}}$ & $a_{C R}$ \\
\hline Kano OL & 1.97 & 6.98 & 4.38 & 8.87 & 0.88 & -0.50 & 0.004 & 0.23 & 0.36 \\
Kano CL & 1.86 & 7.97 & 3.85 & 7.48 & 9.65 & 0.23 & 1.94 & 0.05 & 0.19 \\
\hline \multicolumn{1}{c}{ Source: from Author. }
\end{tabular}

OL: model with open loop estimated parameters and CL: model with closed loop estimated parameters.

Table 6.17 - ISMC tunings in the teflon gasket valve test.

\begin{tabular}{lrrrr} 
Model & $k_{i s m c}$ & $\lambda_{i s m c}$ & $\Phi_{i s m c}$ & $\tau_{i s m c}$ \\
\hline Kano OL & 25.00 & 1975.35 & 0.75 & 0.18 \\
Kano CL & 776.84 & 9.48 & 1.00 & 0.11 \\
\hline \multicolumn{5}{c}{ Source: from Author. }
\end{tabular}

OL: model with open loop estimated parameters and CL: model with closed loop estimated parameters. 
Table 6.18 - FFPID tunings in the teflon gasket valve test.

\begin{tabular}{lrrrrrr} 
Model & $K_{p}$ & $K_{i}$ & $K_{d}$ & $\tau_{d f}$ & $e_{I_{t h}}$ & $k_{f f}$ \\
\hline Kano OL & 3.20 & 1.18 & 0.41 & 0.08 & 0.09 & 0.77 \\
Kano CL & 2.31 & 1.84 & 0.16 & 0.06 & 0.001 & 0.64 \\
\hline \multicolumn{7}{c}{ Source: from Author. }
\end{tabular}

OL: model with open loop estimated parameters and CL: model with closed loop estimated parameters.

The performed tests are the same presented in Section 6.2 and the performance comparison among the different tunings is presented in Figures 6.44 to 6.46. The best performances are achieved when using the ISMC controller, followed by the PID with CF and CR controller, both tuned using the AIC framework. The drawback regarding the ISMC controller is its high control effort, that is at least one order of magnitude higher than the PID with CF and CR. In general, using an open or closed loop estimated model in the AIC framework does not affect greatly the controller tuning performance. Yet, the DS method presents lower performances than the AIC framework, which is not surprising, as the former utilizes a simplified plant model.

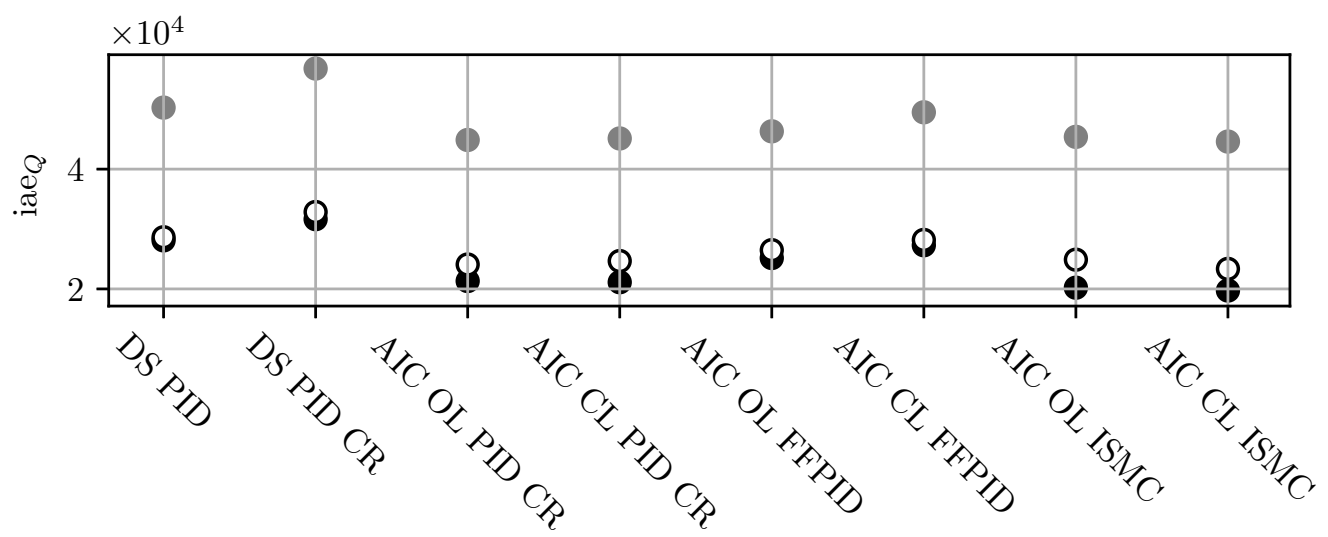

Figure 6.44 - Comparison of the $\mathrm{iae}_{Q}$ index, in the teflon gasket valve test. Legend: regulatory (white), servo (black) and aleatory (gray) experiments.

Source: from Author. 


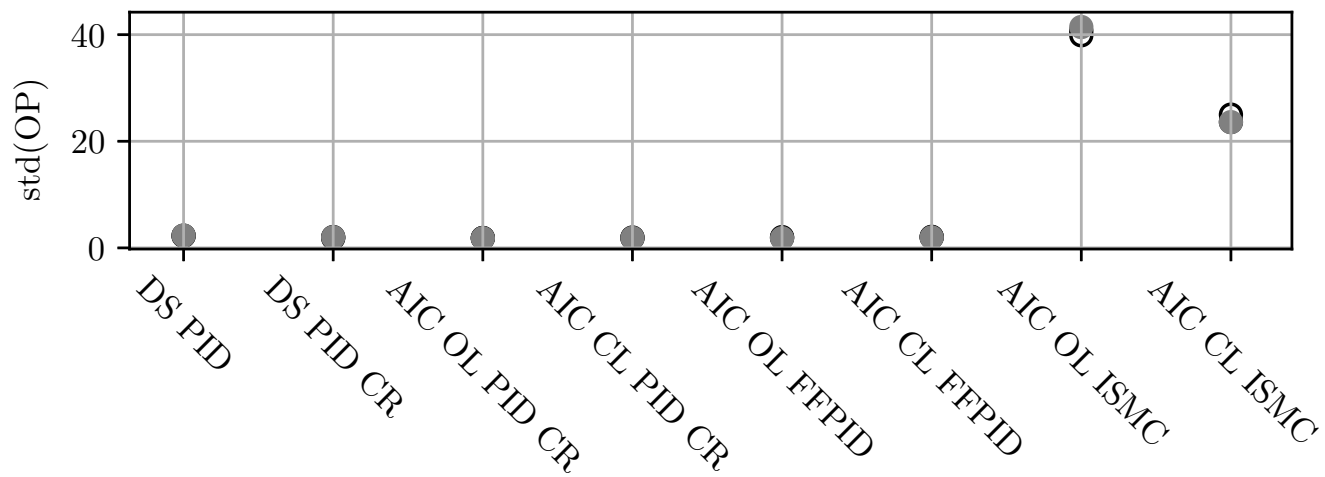

Figure 6.45 - Comparison of the std(OP) index, in the teflon gasket valve test. Legend: regulatory (white), servo (black) and aleatory (gray) experiments. Source: from Author.

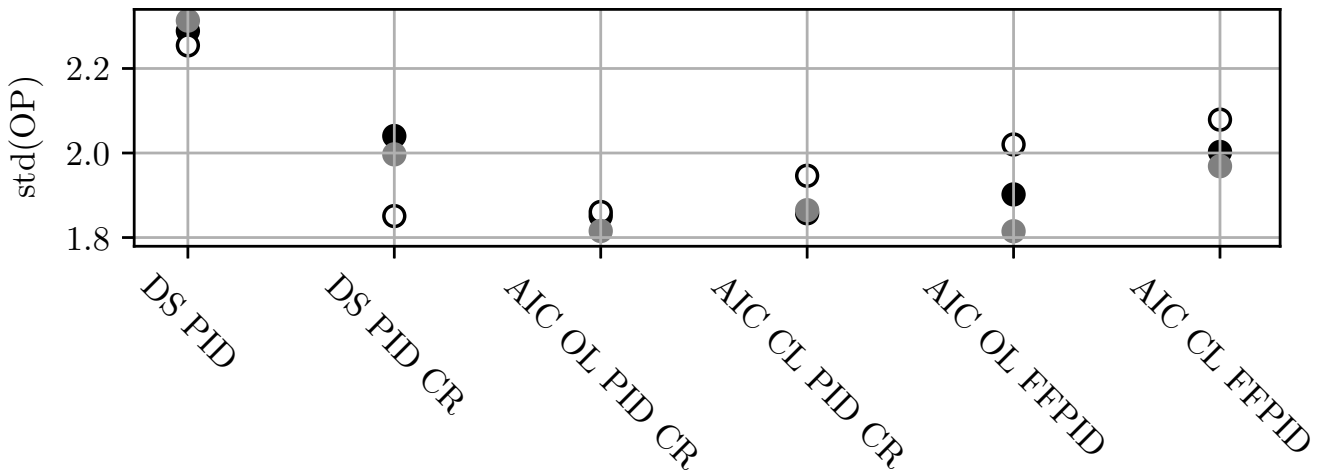

Figure 6.46 - Comparison of the std(OP) index, excluding the ISMC controller. Legend: regulatory (white), servo (black) and aleatory (gray) experiments.

Source: from Author.

For a time domain performance comparison between the controllers, an extract from the servo experiment is presented in Figures 6.47 to 6.50.

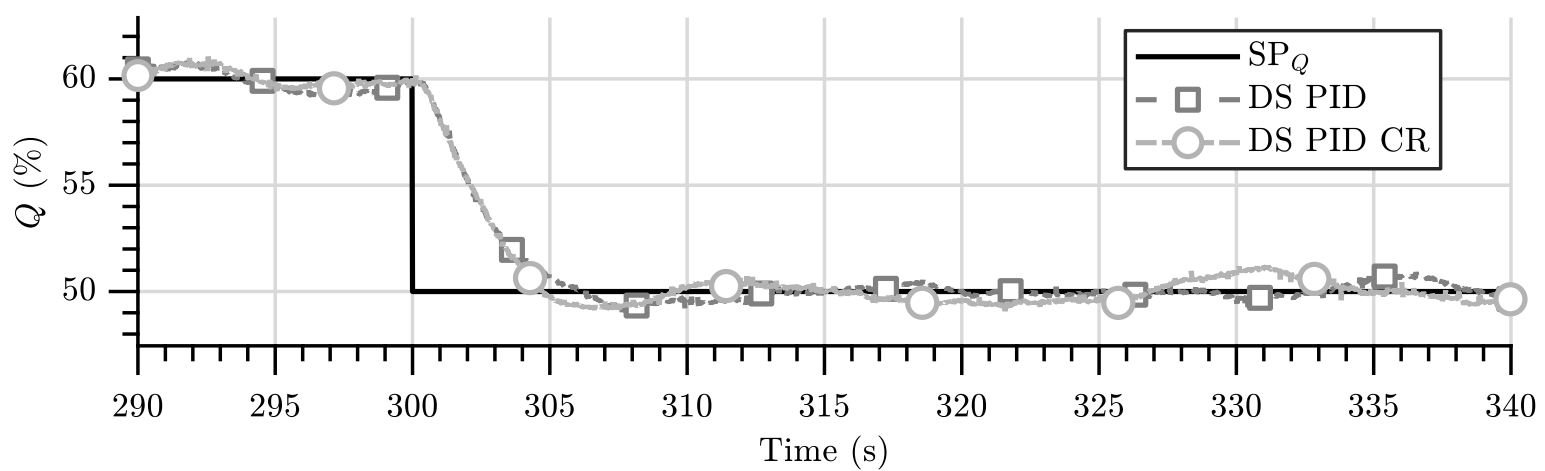

Figure 6.47 - Extract of the servo experiment using the PID and PID with CF and CR, tuned using the DS method.

Source: from Author. 


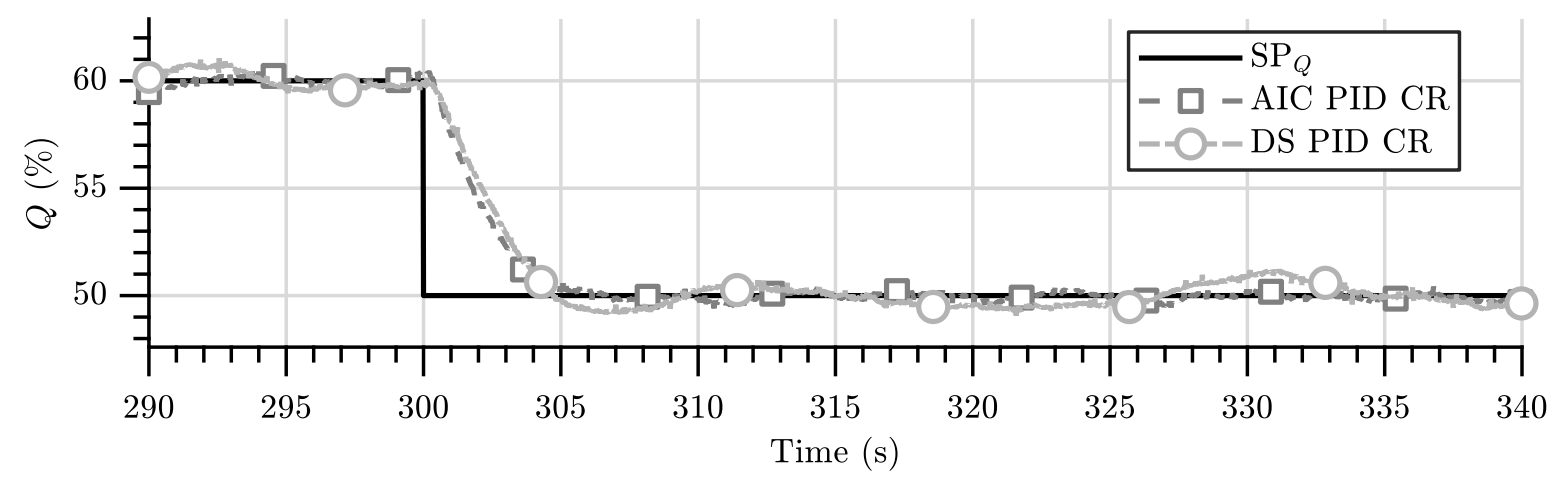

Figure 6.48 - Extract of the servo experiment using the PID with CF and CR, tuned using the DS method and the AIC with the open loop estimated Kano model.

Source: from Author.

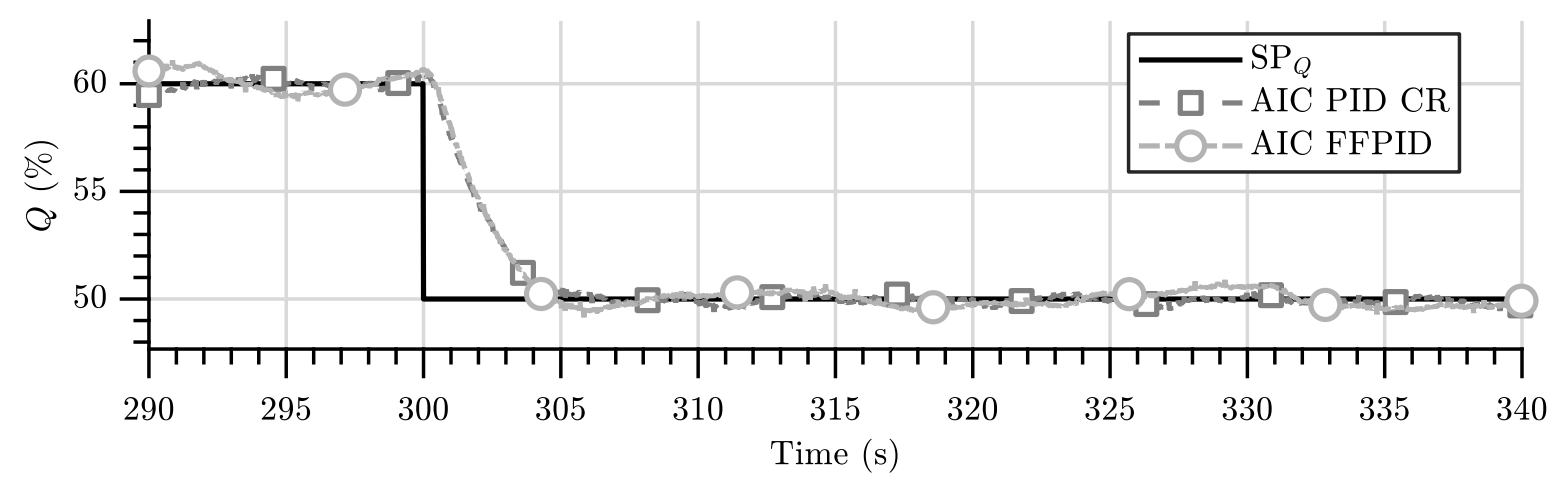

Figure 6.49 - Extract of the servo experiment using the PID with CF and CR and FFPID, tuned using the the AIC with the open loop estimated Kano model.

Source: from Author.

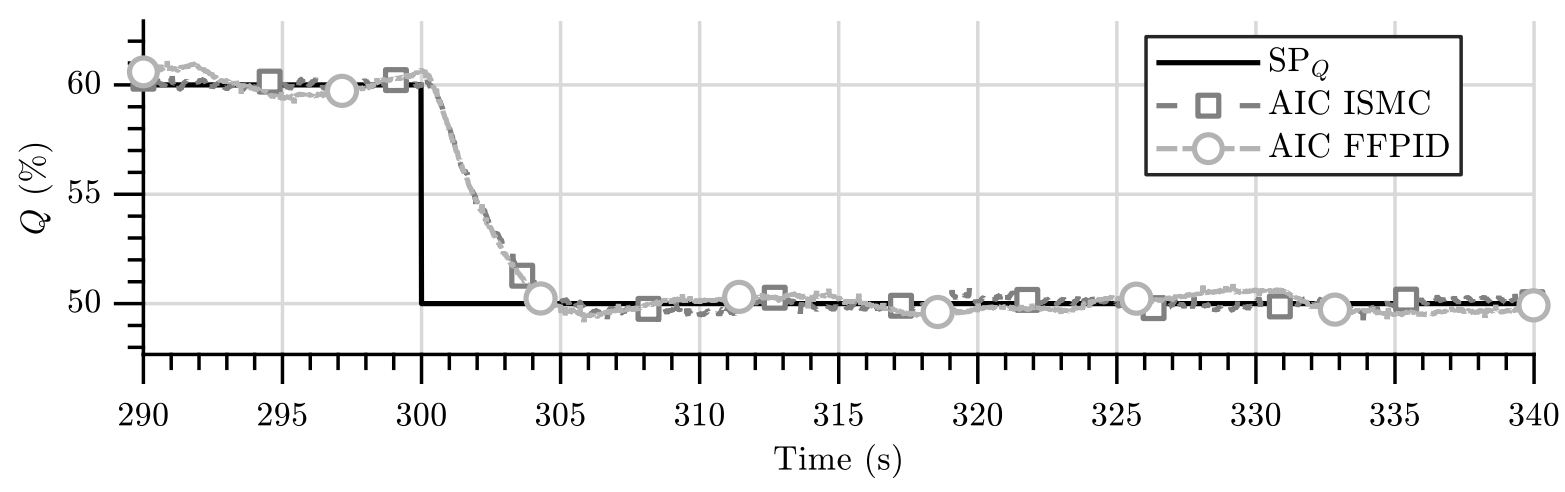

Figure 6.50 - Extract of the servo experiment using the FFPID and ISMC, tuned using the the AIC with the open loop estimated Kano model.

Source: from Author. 


\subsection{Concluding Remarks}

In this chapter, the automatic friction compensation using the AIC framework was designed. If the developments of Chapters 4,5 and 6 are combined, then it is possible to monitor the valve behavior during normal operation and if a deviation occurs, for example due to an increase in a friction parameter, then it is possible to estimate a new valve model and then automatically tune a given controller structure to compensate for the changes in the valve dynamics.

Choosing a friction model to use in the AIC framework is not an easy task, as using a more complex friction model does not deterministically result in better tuning performances. By viewing the experiments individually, grouped by the type of estimated model that is used (e.g. closed or open loop estimated models) and by experiment type, then the GMS, Kano and Karnopp tuned controllers presented the best results in 21, 9 and 12 experiments, respectively, accounting for all performed tests. Except for when using the $\hat{\tau}_{\mathrm{I} / \mathrm{P}_{s}}$ as the I/P model time constant, if the GMS model is used in the AIC framework, the tuning performance is very consistent, with little impact whether the model is estimated in open or closed loop. When the Kano and Karnopp models are used in the AIC framework instead, the performance tend to vary if the valve model is estimated in open or closed loop. The GMS model is specially effective to tune the CF method, as shown in Section 6.3. If performance and consistency is the controller first objective and there is a computationally powerful processor available, then it is preferable to use the GMS model. If the processing power has to be minimized, then the Karnopp and Kano models can be used. Still, even if the GMS or Karnopp model is used, as they tend to be much slower than the Kano model, the latter can be used for a fast valve model estimation and controller tuning, until the more complex model is being calculated.

With regard to the controller structure, even though the FFPID and ISMC are more efficient in some test cases, the PID with CF and CR is very consistent, has a simpler control structure and presents lower control effort. Therefore, the tests show that the PID with $\mathrm{CF}$ and $\mathrm{CR}$ is the preferable controller structure for diaphragm control valves with digital positioners.

In general, using the AIC framework is more effective for tuning the controllers than using the DS method with friction compensation algorithms, configured as suggested in the literature. 


\section{Conclusions}

The algorithms and guidelines for estimating the valve with friction model in open or closed loop systems was derived for the Kano, Karnopp, LuGre and GMS friction models. From simulated tests, only the Karnopp model is estimated perfectly when measurement noise is simulated. In experimental tests, however, the GMS model tends to have a more precise stem velocity prediction precision. Even though the Karnopp performs quite well also, but may present undesired stick-slip behavior in some particular cases. The LuGre model simulation may fail if the diaphragm pressure has measurement noise and therefore was eliminated from the controller tests. The Kano model, despite being very computationally efficient, has inaccurate predictions, specially when its input signal has noise. In this work, the GMS model has 14 parameters, which makes the estimation process very time consuming, therefore one may consider if the added complexity is worth to obtain slightly better model prediction precisions, when compared to the Karnopp model.

To achieve the automatic friction compensation, the AIC framework was designed to tune some usually employed controller structures that are used to compensate diaphragm valve friction. In general, when the estimated GMS model is used in the AIC framework, the controller tuning performance is more likely to be optimal, however as this is not a deterministic phenomena, in some tests the Karnopp or Kano models provided better tunings.

From the performed modeling and control experiments, it is possible to conclude that the GMS model is more likely to deliver better results over the other models, as it has the most consistent performance. However, the Karnopp model can also produce good results, and may substitute the GMS model without great losses. The Kano model is negatively affected by measurement noise and should not be used to model diaphragm valves, unless the valve has no stem position sensor. Also, the Kano model is more likely to produce poorer controller tunings, when compared to the other models. The LuGre model should be avoided, as it may fail completely if the valve input $(P)$ has noise.

\section{Future Work}

The GMS model used in this work has a high number of parameters, which mainly improve the presliding prediction precision. As this phenomenon is not of interest when modeling diaphragm valves, since the available sensors that are frequently used in the industry to measure the valve stem position do not have micrometric resolution, a simplified version of the GMS model, with only one Maxwell element (resulting in 7 parameters), can be tested to verify whether this simplified GMS model still outperforms the other models. 
In this work, the valve model integration algorithm has a fixed time step. The computational efficiency of the Karnopp, LuGre and GMS models can be greatly improved if an adaptive integration time step algorithm can be developed. The smaller time steps are necessary mainly when the system is in presliding state.

A more precise position sensor can be installed on the valve, to check whether there is a bigger difference between the prediction precision of the friction models. The position sensor can be a linear magnetic encoder, which has micrometric resolution, minimal measurement noise and may be applicable to industrial applications.

The controller structures tested in this work do not use all the available information regarding the estimated friction models, as the FFPID and ISMC controllers use a simplified friction model, since estimating the internal states of the friction models is a difficult task. A more precise test, that has the potential to use all information regarding the estimated models, is to design a Moving Horizon Estimation and a Model Predictive Control for the valve position controller.

The ISMC and the FFPID controllers are sensitive to the sampling time, therefore a hardware that allows lower sampling times can be designed, to test whether the performance of such controllers improves.

The AIC framework can be extended to ensure that the controller tuning is robust with respect to valve model parametric uncertainties. 


\section{References}

AL-BENDER, F.; LAMPAERT, V.; SWEVERS, J. The Generalized Maxwell-Slip Model: A Novel Model for Friction Simulation and Compensation. IEEE Transactions on Automatic Control, v. 50, n. 11, p. 1883-1887, 2005.

ARMSTRONG-HÉLOUVRY, B.; DUPONT, P.; CANUDAS de WIT, C. A Survey of Models, Analysis Tools and Compensation Methods for the Control of Machines with Friction. Automatica, v. 30, n. 7, p. 1083-1138, 1994.

ARORA, R. K. Optimization: Algorithms and Applications. 1. ed. Boca Raton, Florida, USA: CRC Press, 2015.

ÅSTRÖM, K. J.; WITTENMARK, B. Adaptive Control. 1. ed. USA: Addison-Wesley, 1995.

BAEZA, J. R.; GARCIA, C. Friction Compensation in Pneumatic Control Valves Through Feedback Linearization. Journal of Control, Automation and Electrical Systems, v. 29, n. 3, p. 303-317, 2018.

CANUDAS de WIT, C.; LISCHINSKY, P. Adaptive Friction Compensation with Partially Known Dynamic Friction Model. International Journal of Adaptive Control and Signal Processing, v. 11, p. 65-80, 1997.

CANUDAS de WIT, C.; NOEL, P.; AUBIN, A.; BROGLIATO, B. Adaptive Friction Compensation in Robot Manipulators: Low Velocities. The International Journal of Robotics Research, v. 10, n. 3, p. 189-199, 1991.

CANUDAS de WIT, C.; OLSSON, H.; ÅSTRÖM, K. J.; LISCHINSKY, P. A New Model for Control of Systems with Friction. IEEE Transactions on Automatic Control, v. 40, n. 3, p. 419-425, 1995.

CHOUdhurY, M. A. A. S.; JAIN, M.; SHAH, S. L. Stiction - Definition, Modelling, Detection and Quantification. Journal of Process Control, v. 18, n. 3-4, p. 232-243, 2008.

CHOUDHURY, M. A. A. S.; SHAH, S. L.; THORNHILL, N. F.; SHOOK, D. S. Automatic Detection and Quantification of Stiction in Control Valves. Control Engineering Practice, v. 14, n. 12, p. 1395-1412, 2006.

CHOUdhurY, M. A. A. S.; THORNHILL, N. F.; SHAH, S. L. Modelling Valve Stiction. Control Engineering Practice, v. 13, n. 5, p. 641-658, 2005.

CLARKE, F. H.; LEDYAEV, Y. S.; STERN, R. J.; WOLENSKI, R. R. Nonsmooth Analysis and Control Theory. 1. ed. New York, USA: Springer-Verlag, 1998.

CUADROS, M. A. S. L.; MUNARO, C. J.; MUNARETO, S. Novel Model-Free Approach for Stiction Compensation in Control Valves. Industrial and Engineering Chemistry Research, v. 51, n. 25, p. 8465-8476, 2012.

EMERSON. Fisher 657 and 667 Diaphragm Actuators. Iowa, USA: Fisher Control International, 2000. 
EMERSON. Control Valve Handbook. Iowa, USA: Fisher Control International, 2005.

FADALI, M. S.; VISIOLI, A. Digital Control Engineering: Analysis and Design. 2. ed. USA: Elsevier, 2013.

FEEMSTER, M.; VEDAGARBHA, P.; DAWSON, D. M.; HASTE, D. Adaptive Control Techniques for Friction Compensation. Mechatronics, v. 9, p. 125-145, 1999.

FERRAGUD, F. X. B. Control Predictivo Basado en Modelos Mediante Técnicas de Optimización Heurística. Aplicación a Procesos no Lineares y Multivariables. Thesis (Ph.D.) — Universidad Politecnica de Valencia, 1999.

FREIDOVICH, L.; ROBERTSSON, A.; SHIRIAEV, A.; JOHANSSON, R. LuGre-ModelBased Friction Compensation. IEEE Transactions on Control Systems Technology, v. 18, n. 1, p. 194-200, 2010.

GARCIA, C. Friction Model Parameter Estimation for Control Valves. In: 8th International Federation on Automatic Control Symposium on Dynamics and Control of Process Systems. Cancun, Mexico: IFAC, 2007. p. 273-278.

GARCIA, C. Comparison of Friction Models Applied to a Control Valve. Control Engineering Practice, v. 16, n. 10, p. 1231-1243, 2008.

HAGENBLAD, A.; LJUNG, L.; WILLS, A. Maximum Likelihood Identification of Wiener Models. Automatica, v. 44, n. 11, p. 2697-2705, 2008.

HÄGGLUND, T. A Friction Compensator for Pneumatic Control Valves. Journal of Process Control, v. 12, n. 8, p. 897-904, 2002.

HALANAY, A.; IONESCU, V. Time-Varying Discrete Linear Systems. Basel, Switzerland: Springer Basel AG, 1994.

HIDALGO, M. C.; GARCIA, C. Friction Compensation in Control Valves: Nonlinear Control and Usual Approaches. Control Engineering Practice, v. 58, p. 42-53, 2017.

IVAN, L. Z. X.; LAKSHMINARAYANAN, S. A New Unified Approach to Valve Stiction Quantification and Compensation. Industrial and Engineering Chemistry Research, v. 48, n. 7, p. 3474-3483, 2009.

JELALI, M. Estimation of Valve Stiction in Control Loops Using Separable Least-Squares and Global Search Algorithms. Journal of Process Control, v. 18, n. 7-8, p. 632-642, 2008.

KANO, M.; MARUTA, H.; KUGEMOTO, H.; SHIMIZU, K. Practical Model and Detection Algorithm for Valve Stiction. In: Proceedings of the rth IFAC DYCOPS. Boston, USA: IFAC, 2004.

KARNOPP, D. Computer Simulation of Stick-Slip Friction in Mechanical Dynamic Systems. Journal of Dynamic Systems, Measurement, and Control, v. 107, n. 1, p. 100-103, 1985.

KAYIHAN, A.; DOYLE III, F. J. Friction Compensation for a Process Control Valve. Control Engineering Practice, v. 8, p. 799-812, 2000.

KORMYLO, J. J.; JAIN, V. K. Two-Pass Recursive Digital Filter with Zero Phase Shift. IEEE Transactions on Acoustics, Speech, and Signal Processing, v. 22, n. 5, p. 384-387, 1974. 
LJUNG, L. System Identification: Theory for User. 2. ed. Upper Saddle River, NJ, USA: Prentice Hall, 1999.

MARQUES, F. Algorithms and Data for Diaphragm Control Valves Estimation and Automatic Friction Compensation. Zenodo, 2019. Available from Internet: < https://doi. org/10.5281/zenodo.3464682>.

MARQUES, F. G.; GARCIA, C. Stribeck Parameters Estimation of a Diaphragm Valve Using Quasi Newton Method. In: 12th IFAC Symposium on Dynamics and Control of Process Systems including Biosystems. Florianópolis, Brazil: IFAC, 2019.

MÁRTON, L.; LANTOS, B. Modeling, Identification, and Compensation of Stick-Slip Friction. IEEE Transactions on Industrial Electronics, v. 54, n. 1, p. 511-521, 2007.

MISHRA, P.; KUMAR, V.; RANA, K. P. S. A Novel Intelligent Controller for Combating Stiction in Pneumatic Control Valves. Control Engineering Practice, v. 33, p. 94-104, 2014.

MOHAMmAD, M. A.; HUANG, B. Compensation of Control Valve Stiction Through Controller Tuning. Journal of Process Control, v. 22, p. 1800-1819, 2012.

MORA, J. A. A. Modelagem e Simulação de Planta-Piloto de Vazão. Dissertation (Masters) - Universidade de São Paulo, 2014.

NELDER, J. A.; MEAD, R. A Simplex Method for Function Minimization. The Computer Journal, v. 7, n. 4, p. 308-313, 1965.

OLSSON, H. Control Systems with Friction. Thesis (Ph.D.) - Lund Institute of Technology, 1996.

OLSSON, H.; ÅSTRÖM, K. J.; Canudas de Wit, C.; GÄFVERT, M.; LISCHINSKY, P. Friction Models and Friction Compensation. European Journal of Control, v. 4, n. 3, p. 176-195, 1998.

PIATKOWSKI, T. GMS Friction Model Approximation. Mechanism and Machine Theory, v. 75 , p. 1-11, 2014.

RIZOS, D. D.; FASSOIS, S. D. Friction Identification Based Upon the LuGre and Maxwell Slip Models. IEEE Transactions on Control Systems Technology, v. 17, n. 1, p. 153-160, 2009.

ROMAnO, R. A.; GARCIA, C. Comparison Between Two Friction Model Parameter Estimation Methods Applied to Control Valves. IFAC Proceedings Volumes, v. 40, n. 5, p. 303-308, 2007.

ROMANO, R. A.; GARCIA, C. Karnopp Friction Model Identification for a Real Control Valve. IFAC Proceedings Volumes, v. 41, n. 2, p. 14906-14911, 2008.

ROMANO, R. A.; GARCIA, C. Valve Friction and Nonlinear Process Model Closed-Loop Identification. Journal of Process Control, v. 21, p. 667-677, 2011.

SILVA, B. C.; GARCIA, C. Comparison of Stiction Compensation Methods Applied to Control Valves. Industrial and Engineering Chemistry Research, v. 53, n. 10, p. 3974-3984, 2014 . 
SOBCZYK, M. R.; GERVINI, V. I.; PERONDI, E. A.; CUNHA, M. A. B. A Continuous Version of the LuGre Friction Model Applied to the Adaptive Control of a Pneumatic Servo System. Journal of the Franklin Institute, v. 353, p. 3021-3039, 2016.

SRINIVASAN, R.; RENGASWAMY, R. Approaches for Efficient Stiction Compensation in Process Control Valves. Computers and Chemical Engineering, v. 32, n. 1-2, p. 218-229, 2008.

STORN, R.; PRICE, K. Differential Evolution - A Simple and Efficient Heuristic for Global Optimization Over Continuous Spaces. Journal of Global Optimization, v. 11, n. 4, p. 341-359, 1997.

SWEVERS, J.; AL-BENDER, F.; GANSEMAN, C. G.; PRAJOGO, T. An Integrated Friction Model Structure with Improved Presliding Behavior for Accurate Friction Compensation. IEEE Transactions on Automatic Control, v. 45, n. 4, p. 675-686, 2000.

THORNHILL, N. F.; HORCH, A. Advances and New Directions in Plant-Wide Disturbance Detection and Diagnosis. Control Engineering Practice, v. 15, n. 10, p. 1196-1206, 2007.

TJAHJOWIDODO, T.; BRUSSEL, H. V. Friction Identification and Compensation in a DC Motor. In: 16th IFAC World Congress. Prague, Czech Republic: IFAC, 2005.

UEHARA, D.; GARCIA, C.; ROMANO, R. A. Comparação e Equivalência dos Modelos de Atrito de Kano e Karnopp Aplicados a Válvulas de Controle. In: 17th Congresso Brasileiro de Automática. Juiz de Fora, Brazil: CBA, 2008.

VERBERT, K. A. J.; TÓTH, R.; BABUSKA, R. Adaptive Friction Compensation: A Globally Stable Approach. IEEE/ASME Transactions on Mechatronics, v. 21, n. 1, p. 351-363, 2016.

WIDROW, B.; WALACH, E. Adaptive Inverse Control. 1. ed. Piscataway, NJ, USA: IEEE Press, 2008.

WORDEN, K.; WONG, C. X.; PARLITZ, U.; HORNSTEIN, A.; ENGSTER, D.; TJAHJOWIDODO, T.; AL-BENDER, F.; RIZOS, D. D.; FASSOIS, S. D. Identification of Pre-Sliding and Sliding Friction Dynamics: Grey Box and Black-Box Models. Mechanical Systems and Signal Processing, v. 21, p. 514-534, 2007. 
Appendices 


\section{APPENDIX A - Optimization Algorithms}

This appendix groups the optimization algorithms used in this thesis, namely: the genetic algorithm, differential evolution, Monte Carlo with simplex, successive nonlinear linear regression and the grid search with differential evolution and simplex optimization.

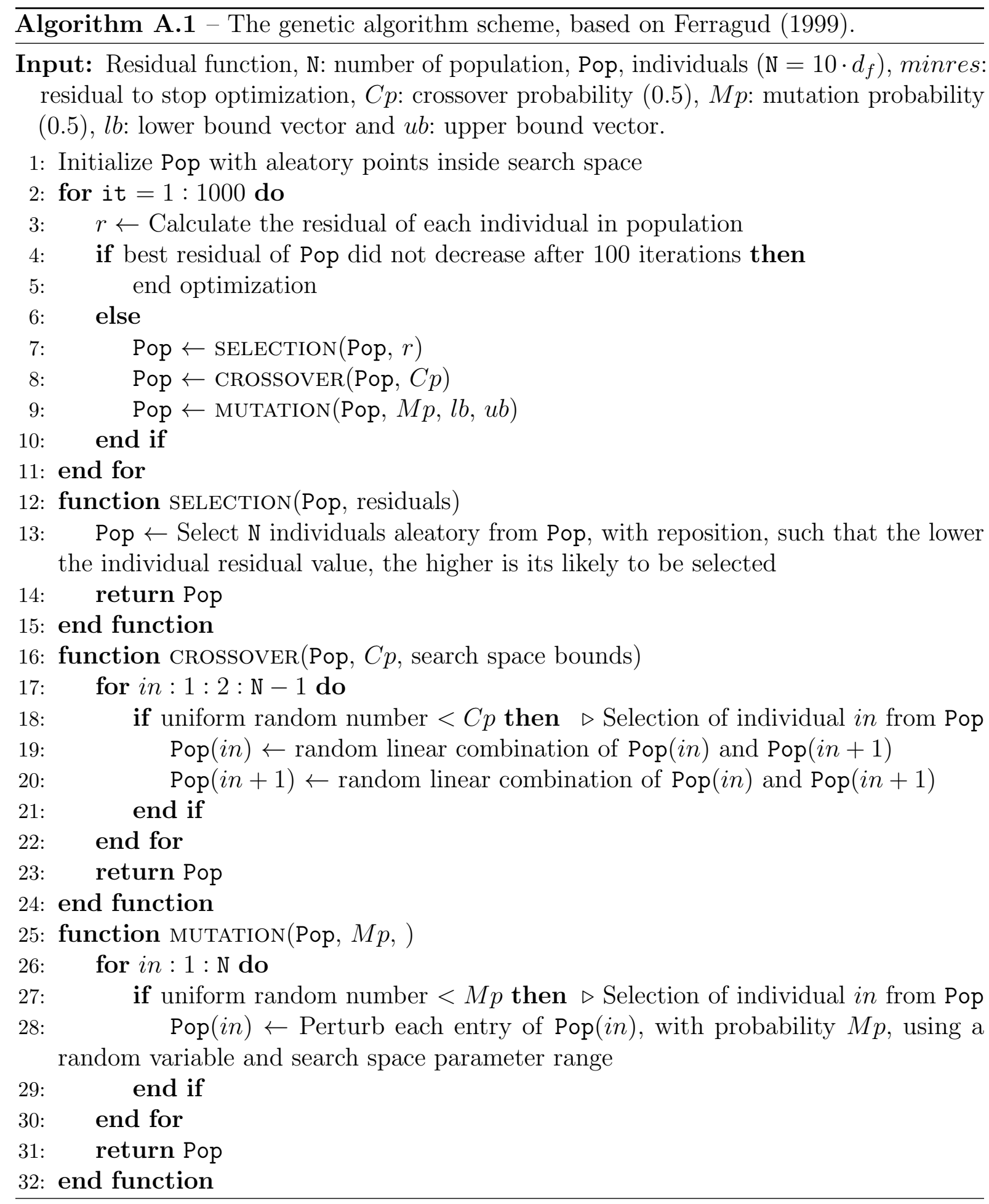




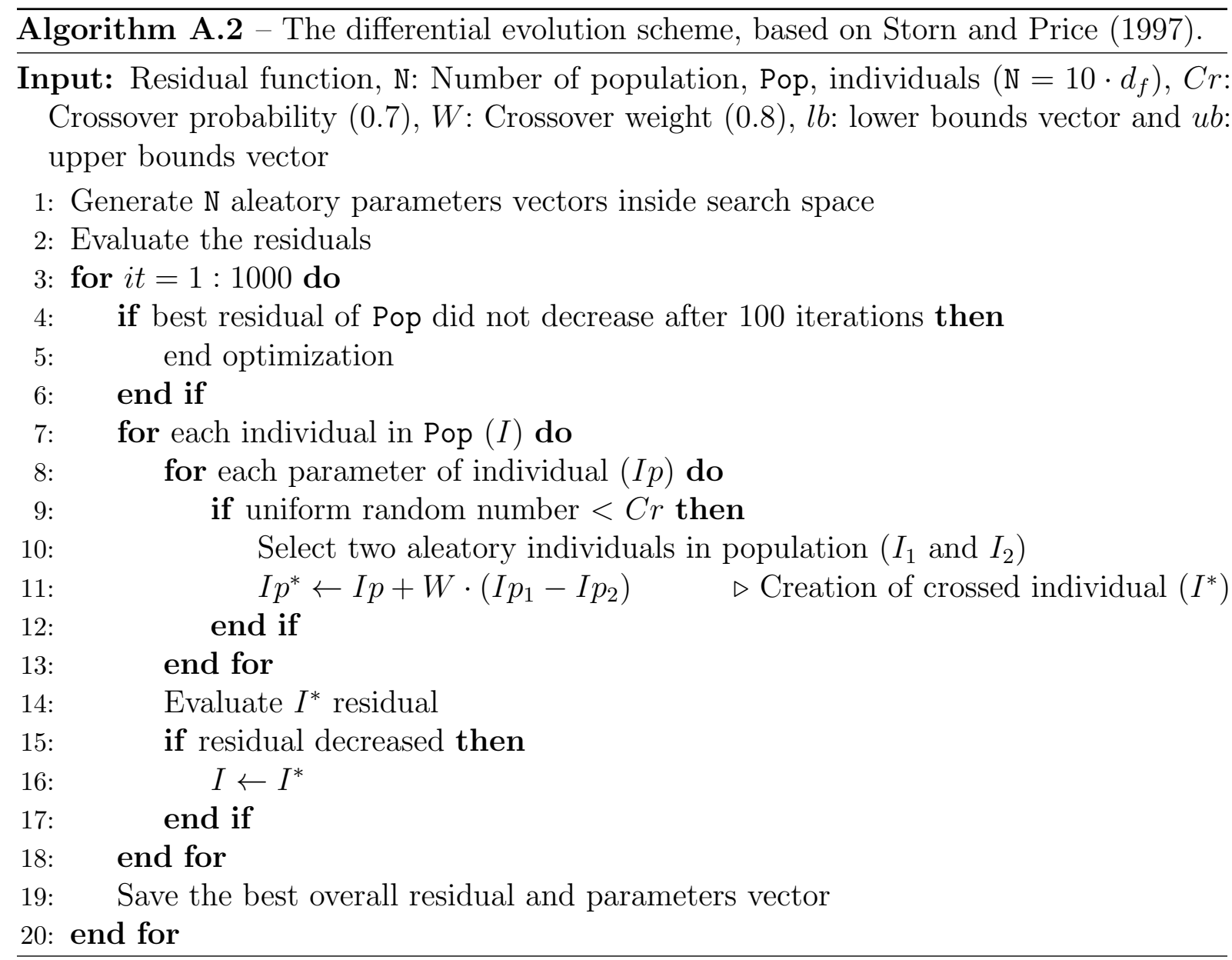

Algorithm A.3 - The Monte Carlo with Simplex optimization algorithm.

Input: Residual function, search space bounds

1: Pop $\leftarrow$ Select a population of $\min \left(40 \cdot 2^{d_{f}}, 60000\right)$ aleatory parameters vector from the search space

2: Calculate the residuals of each element in Pop

3: Select the best $3 \%$ residuals from Pop and use its corresponding parameters vector as the initial point for a Simplex optimization with 200 iterations

4: The parameters vector that resulted in the best residual from previous step is used in a Simplex optimization with 1500 iterations 
Algorithm A.4 - The Simplex scheme, based on the work of Nelder and Mead (1965) and the MATLAB ${ }^{\circledR}$ implementation, modified to run in parallel.

Input: Residual function, initial parameters vector $(\theta)$ with dimension $\mathrm{D}$, maxres: maximum accepted distance between residuals, maxdist: maximum accepted distance between parameters vectors values.

1: Generate D new parameters vectors by perturbing $\theta$ with $5 \%$ magnitude

2: Evaluate the residuals at each $\mathrm{D}+1$ points

3: for $i t=1: 1000$ do

4: $\quad \theta_{r} \leftarrow$ Calculate the average of the best $\mathrm{D}$ residuals $\left(\theta_{a v}\right)$ and reflect the parameter that resulted in the worst residual $\left(\theta_{\text {worst }}\right)$ with respect to the average

5: $\quad \theta_{e} \leftarrow$ Double the distance between $\theta_{r}$ and $\theta_{a v}$

6: $\quad \theta_{c} \leftarrow$ Halve the distance between $\theta_{r}$ and $\theta_{a v}$

7: $\quad \theta_{i c} \leftarrow$ Halve the distance between $\theta_{\text {worst }}$ and $\theta_{a v}$

8: $\quad$ Evaluate the residuals with $\theta_{r}, \theta_{e}, \theta_{c}$ and $\theta_{i c}$

9: $\quad$ if $\theta_{r}$ has the best residual then

10: $\quad$ if $\theta_{e}$ has the best residual then

11:

12 :

$13:$

14:

$$
\theta_{\text {worst }} \leftarrow \theta_{e}
$$

else

$\theta_{\text {worst }} \leftarrow \theta_{r}$ end if else

if $\theta_{r}$ residual is lower than the second worst residual then

$$
\theta_{\text {worst }} \leftarrow \theta_{r}
$$

else

if $\theta_{r}$ residual is lower than $\theta_{\text {worst }}$ then

if $\theta_{c}$ residual is lower than with $\theta_{r}$ then

$$
\theta_{\text {worst }} \leftarrow \theta_{r}
$$

else

SHRINK(parameters vectors)

$$
\text { end if }
$$

else

if $\theta_{i c}$ residual is lower than with $\theta_{\text {worst }}$ then $\theta_{\text {worst }} \leftarrow \theta_{i c}$

else

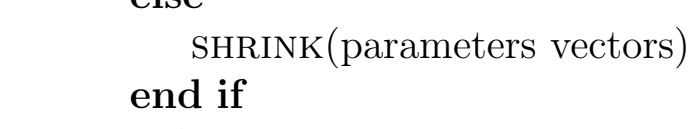

end if

end if

\section{end if}

if no decrease in best residual after 100 iterations then end optimization

\section{end if}

37: $\quad$ if distance between best residual and other residuals $<$ maxres and distance between parameters vectors $<$ maxdist then

38:

39: end optimization

40: end for 
41: function SHRINK(parameters vectors)

42: Halve the distance between the parameters vectors with D worst residuals and the parameters vector with best residual

43: $\quad$ Evaluate the new residuals

44: end function

$\overline{\text { Algorithm A.5 - The successive nonlinear linear regression scheme, based on Rizos and }}$ Fassois (2009).

Input: Residual function, search space bounds, $\operatorname{lin}_{n}$ : number of linear parameters, nlin $_{n}$ : number of nonlinear parameters, $l b$ : lower bounds vector and $u b$ : upper bounds vector.

1: Pop $\leftarrow$ Select a population of $500 \cdot$ nlin $_{n}$ aleatory parameters vector inside the search space

2: Calculate the residuals of each element in Pop

3: lin parameters $\leftarrow$ Select the parameters vector with best residual from Pop

4: Calculate the experimental data $F_{f}$

5: PopGA $\leftarrow$ select the best $10 \cdot$ nlin $_{n}$ residuals nonlinear parameters from Pop

6: Start the initial population of the GA algorithm with PopGA

7: for $i t=1: 1000$ do

8: $\quad$ if $i t>1$ then

9: $\quad$ Update the PopGA using previous it nlin parameters

10: $\quad$ end if

11: nlin parameters $\leftarrow$ Run GA with 20 iterations, using PopGA and fixed lin parameters

12: $\quad$ Simulate the valve model using nlin parameters and lin parameters

13: $\quad$ lin parameters $\leftarrow$ solve LSE using valve simulated data and $F_{f}$

14: $\quad$ if no decrease in best residual after 100 iterations then

15: $\quad$ end optimization

16: end if

17: end for

$\overline{\text { Algorithm A.6 - Grid Search with Differential Evolution and Simplex Optimization }}$ (GDES).

Input: Residual function, search space bounds.

1: From the search space, select points for each parameters and evaluate the residual for all possible combination of parameter points

2: Pop $\leftarrow$ Select the best $10 \cdot d_{f}$ residuals and create an initial population for the DE optimization

3: Pop $\leftarrow$ Perturb the 11th best residual onwards, by adding a normal random variable with standard deviation equal to a hundredth of the parameters bounds

4: Pop $\leftarrow$ Replace the worst 10 parameters with random points inside search space

5: parametersDE $\leftarrow$ Perform a DE optimization with Pop

6: parameters $\leftarrow$ Perform a Simplex optimization using parametersDE as the initial parameters vector 